\title{
Finite Rogers-Ramanujan Type Identities
}

\author{
Andrew V. Sills* \\ through August 2003: \\ Department of Mathematics \\ The Pennsylvania State University, University Park, PA, USA \\ sills@math.psu.edu \\ http://www.math.psu.edu/sills \\ starting September 2003: \\ Department of Mathematics \\ Rutgers University, Hill Center, Busch Campus, Piscataway, NJ, USA \\ sills@math.rutgers.edu \\ http://www. math.rutgers.edu/〜sills
}

Submitted: May 14, 2002; Revised: Aug 27, 2002;

Accepted: Apr 10, 2003; Published: Apr 23, 2003

MR Subject Classifications: 05A10, 11B65

\begin{abstract}
Polynomial generalizations of all 130 of the identities in Slater's list of identities of the Rogers-Ramanujan type are presented. Furthermore, duality relationships among many of the identities are derived. Some of the these polynomial identities were previously known but many are new. The author has implemented much of the finitization process in a Maple package which is available for free download from the author's website.
\end{abstract}

\section{Introduction}

\subsection{Three approaches to finitization}

There are at least three avenues of approach that lead to finite Rogers-Ramanujan type identities.

${ }^{*}$ The research contained herein comprises a substantial portion of the author's doctoral dissertation, submitted in partial fulfillment of the requirements for the Ph.D. degree at the University of Kentucky. The doctoral dissertation was completed under the supervision of George E. Andrews, Evan Pugh Professor of Mathematics at the Pennsylvania State University. This research was partially supported by a grant provided to the author by Professor Andrews. 
1. Combinatorics and models from statistical mechanics. This approach has been studied extensively by Andrews, Baxter, Berkovich, Forrester, McCoy, Schilling, Warnaar and others; see, e.g., [7], [15], [16], [18], [17], [27], [30], [31], [36], [63], [70], [71], [72].

2. The Strong Bailey Lemma. This method is discussed in chapter 3 of Andrews' $q$-series monograph [10].

3. The method of nonhomogeneous q-difference equations. This method is introduced in [10, Chapter 9] and studied extensively herein.

While these three methods sometimes lead to similar results, often the results are different. Even in the cases where the different methods lead to the same finitization, each method has its own inherent interest. For instance, from the statistical mechanics point of view, finitization makes it possible to consider $q \rightarrow q^{-1}$ duality, which in the case of Baxter's hard hexagon model, allows one to neatly pass from one regime to another [7]. Finitizations arising as a result of the application of the strong form of Bailey's Lemma give rise to important questions in computer algebra as in Paule ([52] and [53]). Finally, the method of $q$-difference equations has been studied combinatorially in [9]. It is this method that will be studied in depth in this present work.

Granting the intrinsic merit of all of these approaches, a particularly interesting aspect of the third method stems from the fact that there is no known overarching theory which guarantees a given attempt at finitization will be successful. The fact that all of Slater's list succumbed to this method is evidence in favor of the existence of such an overarching theory.

Let us now begin to study this third method in detail.

\subsection{Overview of this work}

In his monograph on $q$-series [10, Chapter 9], Andrews indicated a method (referred to herein as the "method of first order nonhomogeneous q-difference equations," or more briefly as the "method of $q$-difference equations") to produce sequences of polynomials which converge to the Rogers-Ramanujan identities and identities of similar type. By appropriate application of the $q$-binomial theorem, formulas for the polynomials can easily be produced for what the physicists call "fermionic representations" of the polynomials. The identities explored in [7] and [10] relate to Baxter's solution of the hard hexagon model in statistical mechanics [25]. In [16], Andrews and Baxter suggest some ideas for how a computer algebra system can be employed to find what the physicists call "bosonic representations" of polynomials which converge to Rogers-Ramanujan type products. When we have both a fermionic and bosonic representation of a polynomial sequence which converges to a series-product identity, the series-product identity is said to have been finitized.

In his Ph.D. thesis [60], Santos conjectured bosonic (but no fermionic) representations for polynomial sequences which converge to many of the identities in Lucy Slater's paper on Rogers-Ramanujan Type Identities [68]. 
This present work extends and unifies the results found in [7], [10, Chapter 9], [16] and [60]. Background material is presented in $\S 1$.

In $\S 2$, it is proved that the method of $q$-difference equations can be used to algorithmically produce polynomial generalizations of Rogers-Ramanujan type series, and find fermionic representations of them. As in [16] and [60], bosonic representations need to be conjectured, but the methods and computer algebra tools discussed in $\S 2$ indicate how appropriate conjectures can be found efficiently.

In $\S 3$, at least one finitization is presented for each of the 130 identities in Slater's list [68]. In the case of some of the simpler identities in Slater's list, the finitization found corresponds to a previously known polynomial identity, but in many of the cases, the identities found are new. Considerable care was taken to provide appropriate references for the previously known, and previously conjectured identities or pieces of identities. In each case, the bosonic representations can best be understood in terms of either Gaussian polynomials or $q$-trinomial coëfficients [15]. Particularly noteworthy is the discovery that bosonic representations of a number of the finitized Slater identities used a weighted combination of two different $q$-trinomial coëfficients, referred to herein as $\mathrm{V}(L, A ; q)$ (see (1.23)). It turns out that this " $V$ " function enters naturally into the theory of $q$-trinomial coëfficients due to certain internal symmetries of the $T_{0}$ and $T_{1} q$-trinomial coëfficients (1.33), although its existence had previously gone unnoticed.

Section 4 contains a discussion of various methods for proving the polynomial identities conjectured by the method of $q$-difference equations. Particular emphasis is placed upon the algorithmic proof theory of Wilf and Zeilberger ([55], [76], [77], [78], [79], [80]). It is to be noted that the author has proved every identity in $\S 3$ using the "method of recurrence proof" discussed in Section 4, including the 1991 Santos conjectures, as well as new polynomial identities. Thus, all of the identities in Slater's list [68] may now be viewed as corollaries of the polynomial identities presented in $\S 3$.

Once a series-product identity is finitized, a $q \rightarrow q^{-1}$ duality theory can be discussed. In [7], Andrews describes the duality between various identities associated with the four regimes of the hard hexagon model. An extensive study of the duality relationships among the identities presented in $\S 3$ is undertaken in $\S 5$. A number of previously unknown multisum identities arise as a result of this duality study.

In $\S 6$, a relaxed version of the finitization method of $\S 2$ is considered wherein we drop the requirement that the two-variable generalization of the Rogers-Ramanujan type series satisfy a first order nonhomogeneous $q$-difference equation. It is then demonstrated that this method can be used to find several identities due to Bressoud [32], as well as to find additional new finitizations of Rogers-Ramanujan type identities, at least one of which arises in the work of Warnaar [72].

Finally, the appendix is an annotated and cross-referenced version of Slater's list of identities from [68]. Since Slater's list of identities has been the source for further research for many mathematicians, my hope is that others will find this version of Slater's list useful. 


\section{Background Material}

\section{$1.1 q$-Binomial coëfficients}

We define the infinite rising $q$-factorial $(a ; q)_{\infty}$ as follows:

$$
(a ; q)_{\infty}:=\prod_{m=0}^{\infty}\left(1-a q^{m}\right),
$$

where $a$ and $q$ may be thought of as complex numbers, and then the finite rising $q$-factorial $(a ; q)_{n}$ by

$$
(a ; q)_{n}:=\frac{(a ; q)_{\infty}}{\left(a q^{n} ; q\right)_{\infty}}
$$

for all complex $n, a$, and $q$. Thus, if $n$ is a positive integer,

$$
(a ; q)_{n}=\prod_{m=0}^{n-1}\left(1-a q^{m}\right)
$$

In the $q$-factorials $(a ; q)_{n}$ and $(a ; q)_{\infty}$, the " $q$ " is referred to as the "base" of the factorial. It will often be convenient to abbreviate a product of rising $q$-factorials with a common base

$$
\left(a_{1} ; q\right)_{\infty}\left(a_{2} ; q\right)_{\infty}\left(a_{3} ; q\right)_{\infty} \ldots\left(a_{r} ; q\right)_{\infty}
$$

by the more compact notation

$$
\left(a_{1}, a_{2}, a_{3}, \ldots, a_{r} ; q\right)_{\infty}
$$

The Gaussian polynomial $\left[\begin{array}{l}A \\ B\end{array}\right]_{q}$ may be defined ${ }^{1}$ :

$$
\left[\begin{array}{l}
A \\
B
\end{array}\right]_{q}:= \begin{cases}(q ; q)_{A}(q ; q)_{B}^{-1}(q ; q)_{A-B}^{-1}, & \text { if } 0 \leqq B \leqq A \\
0, & \text { otherwise. }\end{cases}
$$

Note that even though the Gaussian polynomial $\left[{ }_{B}^{A}\right]_{q}$ is defined as a rational function, it does, in fact, reduce to a polynomial for all integers $A, B$, just as the fraction $\frac{A !}{B !(A-B) !}$ simplifies to an integer. Notice that in the case where $A$ and $B$ are positive integers with $B \leqq A$

$$
\left[\begin{array}{l}
A \\
B
\end{array}\right]_{q}=\frac{\left(1-q^{A}\right)\left(1-q^{A-1}\right)\left(1-q^{A-2}\right) \cdots\left(1-q^{A-B+1}\right)}{(1-q)\left(1-q^{2}\right)\left(1-q^{3}\right) \cdots\left(1-q^{B}\right)}
$$

and so

$$
\operatorname{deg}\left(\left[\begin{array}{l}
A \\
B
\end{array}\right]_{q}\right)=B(A-B)
$$

\footnotetext{
${ }^{1}$ Variations of this definition are possible for $B<0$ or $B>A$; see, e.g. Berkovich, McCoy, and Orrick [30, p. 797, eqn. (1.7)] for a variation frequently used in statistical mechanics.
} 


$$
\lim _{q \rightarrow 1}\left[\begin{array}{l}
A \\
B
\end{array}\right]_{q}=\left(\begin{array}{l}
A \\
B
\end{array}\right)
$$

where $\left(\begin{array}{l}A \\ B\end{array}\right)$ is the ordinary binomial coëfficient, thus Gaussian polynomials are also called q-binomial coëfficients.

Just as ordinary binomial coëfficients satsify the symmetry relationship

$$
\left(\begin{array}{l}
A \\
B
\end{array}\right)=\left(\begin{array}{c}
A \\
A-B
\end{array}\right)
$$

so do Gaussian polynomials satisfy the symmetry relationship

$$
\left[\begin{array}{l}
A \\
B
\end{array}\right]_{q}=\left[\begin{array}{c}
A \\
A-B
\end{array}\right]_{q}
$$

Likewise, the Pascal triangle recurrence

$$
\left(\begin{array}{l}
A \\
B
\end{array}\right)=\left(\begin{array}{l}
A-1 \\
B-1
\end{array}\right)+\left(\begin{array}{c}
A-1 \\
B
\end{array}\right)
$$

has two q-analogs:

$$
\begin{gathered}
{\left[\begin{array}{l}
A \\
B
\end{array}\right]_{q}=\left[\begin{array}{c}
A-1 \\
B
\end{array}\right]_{q}+q^{A-B}\left[\begin{array}{l}
A-1 \\
B-1
\end{array}\right]_{q}} \\
{\left[\begin{array}{l}
A \\
B
\end{array}\right]_{q}=\left[\begin{array}{l}
A-1 \\
B-1
\end{array}\right]_{q}+q^{B}\left[\begin{array}{c}
A-1 \\
B
\end{array}\right]_{q}}
\end{gathered}
$$

for $A>0$ and $0 \leqq B \leqq A$. For a complete discussion and proofs of (1.4) - (1.6), see Andrews [6, pp. $305 \mathrm{ff}]$.

We also record the easily established identity

$$
\left[\begin{array}{l}
A \\
B
\end{array}\right]_{1 / q}=q^{B(B-A)}\left[\begin{array}{l}
A \\
B
\end{array}\right]_{q}
$$

and the asymptotic result

$$
\lim _{n \rightarrow \infty}\left[\begin{array}{c}
2 n+a \\
n+b
\end{array}\right]_{q}=\frac{1}{(q ; q)_{\infty}} .
$$

The binomial theorem may be stated as

$$
\sum_{j=0}^{\infty}\left(\begin{array}{c}
L \\
j
\end{array}\right) t^{j}=(1+t)^{L}
$$

The $q$-binomial theorem, which seems to have been discovered independently by Cauchy [33], Heine [44], and Gauss [39], follows: 
q-Binomial Theorem. [14, p. 488, Thm. 10.2.1] or [6, p. 17, Thm. 2.1]. If $|t|<1$ and $|q|<1$,

$$
\sum_{k=0}^{\infty} \frac{(a ; q)_{k}}{(q ; q)_{k}} t^{k}=\frac{(a t ; q)_{\infty}}{(t ; q)_{\infty}}
$$

We will make use of the following two corollaries of (1.9): The first corollary, which appears to be due to H. A. Rothe [59],

$$
\sum_{k=0}^{j}\left[\begin{array}{l}
j \\
k
\end{array}\right]_{q}(-1)^{k} q^{\left(\begin{array}{c}
k \\
2
\end{array}\right)} t^{k}=(t ; q)_{j}
$$

may be obtained from (1.9) by setting $a=q^{-j}$. The second corollary,

$$
\sum_{k=0}^{\infty}\left[\begin{array}{c}
j+k-1 \\
k
\end{array}\right]_{q} t^{k}=\frac{1}{(t ; q)_{j}},
$$

is the case $a=q^{j}$ of (1.9). If in (1.10), we replace $q$ by $q^{2 r}$, set $t=-q^{r+s}$ and let $j \rightarrow \infty$, we obtain

$$
\sum_{k=0}^{\infty} \frac{q^{r k^{2}+s k}}{\left(q^{2 r} ; q^{2 r}\right)_{k}}=\left(-q^{r+s} ; q^{2 r}\right)_{\infty}
$$

a formula useful for simplifying certain multisums.

\section{$1.2 \quad q$-Trinomial coëfficients}

\subsubsection{Definitions}

Consider the Laurent polynomial $\left(1+x+x^{-1}\right)^{L}$. Analogous to the binomial theorem, we find

$$
\left(1+x+x^{-1}\right)^{L}=\sum_{j=-L}^{L}\left(\begin{array}{c}
L \\
j
\end{array}\right)_{2} x^{j}
$$

where

$$
\begin{aligned}
\left(\begin{array}{l}
L \\
A
\end{array}\right)_{2} & =\sum_{r \geqq 0} \frac{L !}{r !(r+A) !(L-2 r-A) !} \\
& =\sum_{r=0}^{L}(-1)^{r}\left(\begin{array}{c}
L \\
r
\end{array}\right)\left(\begin{array}{c}
2 L-2 r \\
L-A-r
\end{array}\right) .
\end{aligned}
$$

These $\left(\begin{array}{l}L \\ A\end{array}\right)_{2}$ are called trinomial coëfficients, (not to be confused with the coëfficients which arise in the expansion of $(x+y+z)^{L}$, which are also often called trinomial coëfficients). 
The two representations $(1.14)$ and $(1.15)$ of $\left(\begin{array}{l}L \\ A\end{array}\right)_{2}$ give rise to different $q$-analogs due to Andrews and Baxter [15, p. 299, eqns. (2.7)-(2.12)]: ${ }^{2}$

$$
\begin{aligned}
& \left(\begin{array}{c}
L, B ; q \\
A
\end{array}\right)_{2}:=\sum_{r \geqq 0} \frac{q^{r(r+B)}(q ; q)_{L}}{(q ; q)_{r}(q ; q)_{r+A}(q ; q)_{L-2 r-A}}=\sum_{r=0}^{L} q^{r(r+B)}\left[\begin{array}{l}
L \\
r
\end{array}\right]_{q}\left[\begin{array}{l}
L-r \\
r+A
\end{array}\right]_{q} \\
& \mathrm{~T}_{0}(L, A ; q):=\sum_{r=0}^{L}(-1)^{r}\left[\begin{array}{l}
L \\
r
\end{array}\right]_{q^{2}}\left[\begin{array}{c}
2 L-2 r \\
L-A-r
\end{array}\right]_{q} \\
& \mathrm{~T}_{1}(L, A ; q):=\sum_{r=0}^{L}(-q)^{r}\left[\begin{array}{l}
L \\
r
\end{array}\right]_{q^{2}}\left[\begin{array}{c}
2 L-2 r \\
L-A-r
\end{array}\right]_{q}
\end{aligned}
$$

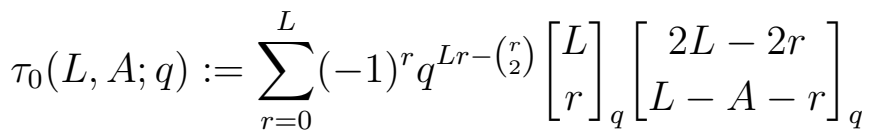

$$
\begin{aligned}
& \mathrm{t}_{0}(L, A ; q):=\sum_{r=0}^{L}(-1)^{r} q^{r^{2}}\left[\begin{array}{l}
L \\
r
\end{array}\right]_{q^{2}}\left[\begin{array}{c}
2 L-2 r \\
L-A-r
\end{array}\right]_{q} \\
& \mathrm{t}_{1}(L, A ; q):=\sum_{r=0}^{L}(-1)^{j} q^{r(r-1)}\left[\begin{array}{l}
L \\
r
\end{array}\right]_{q^{2}}\left[\begin{array}{c}
2 L-2 r \\
L-A-r
\end{array}\right]_{q}
\end{aligned}
$$

It is convenient to follow Andrews [12] and define

$$
\mathrm{U}(L, A ; q):=\mathrm{T}_{0}(L, A ; q)+\mathrm{T}_{0}(L, A+1 ; q) .
$$

Further, I will define

$$
\mathrm{V}(L, A ; q):=\mathrm{T}_{1}(L-1, A ; q)+q^{L-A} \mathrm{~T}_{0}(L-1, A-1 ; q) .
$$

\subsubsection{Recurrences}

The following Pascal triangle type relationship is easily deduced from (1.13):

$$
\left(\begin{array}{l}
L \\
A
\end{array}\right)_{2}=\left(\begin{array}{c}
L-1 \\
A-1
\end{array}\right)_{2}+\left(\begin{array}{c}
L-1 \\
A
\end{array}\right)_{2}+\left(\begin{array}{c}
L-1 \\
A+1
\end{array}\right)_{2} .
$$

${ }^{2}$ Note: Occasionally in the literature (e.g. Andrews and Berkovich [19], or Warnaar [71]), superficially different definitions of the $T_{0}$ and $T_{1}$ functions are used. 
We will require the following $q$-analogs of (1.24), which are due to Andrews and Baxter [15, pp. 300-1, eqns. (2.16), (2.19), (2.25) (2.26), (2.28), and (2.29)]: For $L \geqq 1$,

$$
\begin{aligned}
& \mathrm{T}_{1}(L, A ; q)=\mathrm{T}_{1}(L-1, A ; q)+q^{L+A} \mathrm{~T}_{0}(L-1, A+1 ; q)+q^{L-A} \mathrm{~T}_{0}(L-1, A-1 ; q) \\
& \mathrm{T}_{0}(L, A ; q)=\mathrm{T}_{0}(L-1, A-1 ; q)+q^{L+A} \mathrm{~T}_{1}(L-1, A ; q) \\
& +q^{2 L+2 A} \mathrm{~T}_{0}(L-1, A+1 ; q) \\
& \left(\begin{array}{c}
L, A-1 ; q \\
A
\end{array}\right)_{2}=q^{L-1}\left(\begin{array}{c}
L-1, A-1 ; q \\
A
\end{array}\right)_{2}+q^{A}\left(\begin{array}{c}
L-1, A+1 ; q \\
A+1
\end{array}\right)_{2} \\
& +\left(\begin{array}{c}
L-1, A-1 ; q \\
A-1
\end{array}\right)_{2} \\
& \left(\begin{array}{c}
L, A ; q \\
A
\end{array}\right)_{2}=q^{L-A}\left(\begin{array}{c}
L-1, A-1 ; q \\
A-1
\end{array}\right)_{2}+q^{L-A-1}\left(\begin{array}{c}
L-1, A-1 ; q \\
A
\end{array}\right)_{2} \\
& +\left(\begin{array}{c}
L-1, A+1 ; q \\
A+1
\end{array}\right)_{2} \\
& \left(\begin{array}{c}
L, B ; q \\
A
\end{array}\right)_{2}=\left(\begin{array}{c}
L-1, B ; q \\
A
\end{array}\right)_{2}+q^{L-A-1+B}\left(\begin{array}{c}
L-1, B ; q \\
A+1
\end{array}\right)_{2}+q^{L-A}\left(\begin{array}{c}
L-1, B-1 ; q \\
A-1
\end{array}\right)_{2} \\
& \left(\begin{array}{c}
L, B ; q \\
A
\end{array}\right)_{2}=\left(\begin{array}{c}
L-1, B ; q \\
A
\end{array}\right)_{2}+q^{L-A}\left(\begin{array}{c}
L-1, B-2 ; q \\
A-1
\end{array}\right)_{2}+q^{L+B}\left(\begin{array}{c}
L-1, B+1 ; q \\
A+1
\end{array}\right)_{2}
\end{aligned}
$$

The following identities of Andrews and Baxter [15, p. 301, eqns. (2.20) and (2.27 corrected)], which reduce to the tautology " $0=0$ " in the case where $q=1$ are also useful:

$$
\begin{gathered}
\mathrm{T}_{1}(L, A ; q)-q^{L-A} \mathrm{~T}_{0}(L, A ; q)-\mathrm{T}_{1}(L, A+1 ; q)+q^{L+A+1} \mathrm{~T}_{0}(L, A+1 ; q)=0 \\
\left(\begin{array}{c}
L, A ; q \\
A
\end{array}\right)_{2}+q^{L}\left(\begin{array}{c}
L, A ; q \\
A+1
\end{array}\right)_{2}-\left(\begin{array}{c}
L, A+1 ; q \\
A+1
\end{array}\right)_{2}-q^{L-A}\left(\begin{array}{c}
L, A-1 ; q \\
A
\end{array}\right)_{2}=0 .
\end{gathered}
$$

Observe that (1.31) is equivalent to

$$
\mathrm{V}(L+1, A+1 ; q)=\mathrm{V}(L+1,-A ; q)
$$

The following recurrences appear in Andrews [12, p. 661, Lemmas 4.1 and 4.2]: For $L \geqq 1$

$$
\begin{aligned}
\mathrm{U}(L, A ; q)= & \left(1+q^{2 L-1}\right) \mathrm{U}(L-1, A ; q)+q^{L-A} \mathrm{~T}_{1}(L-1, A-1 ; q) \\
& +q^{L+A+1} \mathrm{~T}_{1}(L-1, A+2 ; q) . \\
\mathrm{U}(L, A ; q)= & \left(1+q+q^{2 L-1}\right) \mathrm{U}(L-1, A ; q)-q \mathrm{U}(L-2, A ; q) \\
& +q^{2 L-2 A} \mathrm{~T}_{0}(L-2, A-2 ; q)+q^{2 L+2 A+2} \mathrm{~T}_{0}(L-2, A+3 ; q) .
\end{aligned}
$$


An analogous recurrence for the "V" function is

$$
\begin{aligned}
\mathrm{V}(L, A ; q)= & \left(1+q^{2 L-2}\right) \mathrm{V}(L-1, A ; q)+q^{L-A} \mathrm{~T}_{0}(L-2, A-2 ; q) \\
& +q^{L+A-1} \mathrm{~T}_{0}(L-2, A+1 ; q) .
\end{aligned}
$$

Proof.

$$
\begin{aligned}
\mathrm{V}(L, A ; q)= & \mathrm{T}_{1}(L-1, A ; q)+q^{L-A} \mathrm{~T}_{0}(L-1, A-1 ; q) \quad(\text { by }(1.23)) \\
= & \mathrm{T}_{1}(L-2, A ; q)+q^{L+A-1} \mathrm{~T}_{0}(L-2, A+1 ; q) \\
& +q^{L-A-1} \mathrm{~T}_{0}(L-2, A-1 ; q)+\mathrm{T}_{0}(L-2, A-2 ; q) \\
& +q^{L+A-2} \mathrm{~T}_{1}(L-2, A-1 ; q)+q^{2 L+2 A-4} \mathrm{~T}_{0}(L-2, A ; q) \quad(\text { by }(1.26 \text { and } 1.25)) \\
= & \mathrm{V}(L-1, A ; q)+q^{L+A-1} \mathrm{~T}_{0}(L-2, A+1 ; q) \\
& +\mathrm{T}_{0}(L-2, A-2 ; q)+q^{L+A-2} \mathrm{~T}_{1}(L-2, A-1 ; q) \\
& +q^{2 L+2 A-4} \mathrm{~T}_{0}(L-2, A ; q) \quad(\text { by }(1.23)) \\
= & \left(1+q^{2 L-2}\right) \mathrm{V}(L-1, A ; q)+q^{L-A} \mathrm{~T}_{0}(L-2, A-2 ; q) \\
& +q^{L+A-1} \mathrm{~T}_{0}(L-2, A+1 ; q) \quad(\text { by }(1.31) \text { and }(1.23)) .
\end{aligned}
$$

\subsubsection{Identities}

From (1.13), it is easy to deduce the symmetry relationship

$$
\left(\begin{array}{l}
L \\
A
\end{array}\right)_{2}=\left(\begin{array}{c}
L \\
-A
\end{array}\right)_{2}
$$

Two $q$-analogs of (1.37) are

$$
\mathrm{T}_{0}(L, A ; q)=\mathrm{T}_{0}(L,-A ; q)
$$

and

$$
\mathrm{T}_{1}(L, A ; q)=\mathrm{T}_{1}(L,-A ; q) .
$$

The analogous relationship for the "round bracket" $q$-trinomial coëfficient ([15, p. 299, eqn. $(2.15)])$ is

$$
\left(\begin{array}{c}
L, B ; q \\
-A
\end{array}\right)_{2}=q^{A(A+B)}\left(\begin{array}{c}
L, B+2 A ; q \\
A
\end{array}\right)_{2} .
$$


Other fundamental relations among the various $q$-trinomial coëfficients include the following (see Andrews and Baxter [15, §2.4, pp. 305-306]):

$$
\begin{gathered}
\left(\begin{array}{c}
L, A ; q \\
A
\end{array}\right)_{2}=\tau_{0}(L, A ; q) \\
\mathrm{T}_{0}\left(L, A ; q^{-1}\right)=q^{A^{2}-L^{2}} \mathrm{t}_{0}(L, A ; q)=q^{A^{2}-L^{2}} \tau_{0}\left(L, A ; q^{2}\right) \\
\mathrm{T}_{1}\left(L, A ; q^{-1}\right)=q^{A^{2}-L^{2}} \mathrm{t}_{1}(L, A ; q) \\
\tau_{0}\left(L, A ; q^{2}\right)=\left(\begin{array}{c}
L, A ; q^{2} \\
A
\end{array}\right)_{2}=\mathrm{t}_{0}(L, A ; q) \\
\left(\begin{array}{c}
L, A-1 ; q^{2} \\
A
\end{array}\right)_{2}=q^{A-L_{1}}(L, A ; q)
\end{gathered}
$$

\subsubsection{Asymptotics}

The following asymptotic results for $q$-trinomial coëfficients are proved in, or are direct consequences of, Andrews and Baxter [15, §2.5, pp. 309-312]:

$$
\begin{gathered}
\lim _{L \rightarrow \infty}\left(\begin{array}{c}
L, A ; q \\
A
\end{array}\right)_{2}=\lim _{L \rightarrow \infty} \tau_{0}(L, A ; q)=\frac{1}{(q ; q)_{\infty}} \\
\lim _{L \rightarrow \infty}\left(\begin{array}{c}
L, A-1 ; q \\
A
\end{array}\right)_{2}=\frac{1+q^{A}}{(q ; q)_{\infty}} \\
\lim _{\substack{L \rightarrow \infty \\
L-A \text { even }}} \mathrm{T}_{0}(L, A ; q)=\frac{\left(-q ; q^{2}\right)_{\infty}+\left(q, q^{2}\right)_{\infty}}{2\left(q^{2} ; q^{2}\right)_{\infty}} \\
\lim _{\substack{L \rightarrow \infty \\
L-A \text { odd }}} \mathrm{T}_{0}(L, A ; q)=\frac{\left(-q ; q^{2}\right)_{\infty}-\left(q ; q^{2}\right)_{\infty}}{2\left(q^{2} ; q^{2}\right)_{\infty}} \\
\lim _{L \rightarrow \infty} \mathrm{T}_{1}(L, A ; q)=\frac{\left(-q^{2} ; q^{2}\right)_{\infty}}{\left(q^{2} ; q^{2}\right)_{\infty}} \\
\lim _{L \rightarrow \infty} \mathrm{V}(L, A ; q)=\frac{\left(-q^{2} ; q^{2}\right)_{\infty}}{\left(q^{2} ; q^{2}\right)_{\infty}} \\
\lim _{L \rightarrow \infty} \mathrm{t}_{0}(L, A ; q)=\frac{1}{\left(q^{2} ; q^{2}\right)_{\infty}} \\
\lim _{L \rightarrow \infty} q^{-L} \mathrm{t}_{1}(L, A ; q)=\frac{q^{-A}+q^{A}}{\left(q^{2} ; q^{2}\right)_{\infty}} \\
\lim _{L \rightarrow \infty} \mathrm{U}(L, A ; q)=\frac{\left(-q ; q^{2}\right)_{\infty}}{\left(q^{2} ; q^{2}\right)_{\infty}}
\end{gathered}
$$

\subsection{Miscellaneous Results}

The following result, found by Jacobi in 1829, is fundamental: 
Jacobi's Triple Product Identity. [6, p. 21, Theorem 2.8] or [45]. For $z \neq 0$ and $|q|<1$,

$$
\begin{aligned}
\sum_{j=-\infty}^{\infty} z^{j} q^{j^{2}} & =\prod_{j=1}^{\infty}\left(1+z q^{2 j-1}\right)\left(1+z^{-1} q^{2 j-1}\right)\left(1-q^{2 j}\right) \\
& =\left(-z q,-z^{-1} q, q^{2} ; q^{2}\right)_{\infty}
\end{aligned}
$$

Note that the asymptotics of the Gaussian polynomials and the $q$-trinomial coëfficients, beside being instances of Jacobi's Triple Product identity, are reciprocals of particular values of $\vartheta$-functions from the classical theory of elliptic functions. If we follow Slater[69, p. $197 \mathrm{ff}$.$] and define$

$$
\begin{gathered}
\vartheta_{2}(z, q):=\sum_{j=-\infty}^{\infty} q^{j+\frac{1}{2}} e^{(2 j+1) i z} \text { and } \\
\vartheta_{4}(z, q):=\sum_{j=-\infty}^{\infty}(-1)^{j} q^{j^{2}} e^{2 j i z},
\end{gathered}
$$

where $i=\sqrt{-1}$, we see that

$$
\begin{gathered}
\vartheta_{4}(0, q)=\sum_{j=-\infty}^{\infty}(-1)^{j} q^{j^{2}}=\frac{(q ; q)_{\infty}}{(-q ; q)_{\infty}}=(q ; q)_{\infty}\left(q ; q^{2}\right)_{\infty}=\frac{1}{\lim _{L \rightarrow \infty} \mathrm{T}_{1}(L, A ; \sqrt{q})}, \\
\vartheta_{2}\left(\pi\left(1-\frac{\tau}{2}\right), q^{3 / 2}\right)=(q ; q)_{\infty}=\frac{1}{\lim _{L \rightarrow \infty} \tau_{0}(L, A ; q)}=\frac{1}{\lim _{L \rightarrow \infty}\left[\begin{array}{l}
2 L+a \\
L+b
\end{array}\right]}, \\
\vartheta_{2}\left(\pi\left(1-\frac{\tau}{2}\right), q^{2}\right)=\frac{\left(q^{2} ; q^{2}\right)_{\infty}}{\left(-q ; q^{2}\right)_{\infty}}=\left(q ; q^{2}\right)_{\infty}\left(q^{4} ; q^{4}\right)_{\infty}=\frac{1}{\lim _{L \rightarrow \infty} \mathrm{U}(L, A ; q)} .
\end{gathered}
$$

where $q=e^{\pi i \tau}$.

The next result, due to Cauchy, is a finite form of (1.55):

$$
\sum_{j=-n}^{n} z^{j} q^{j^{2}}\left[\begin{array}{c}
2 n \\
n+j
\end{array}\right]_{q^{2}}=\left(-z^{-1} q ; q^{2}\right)_{n}\left(-z q ; q^{2}\right)_{n} .
$$

The proof of (1.56) follows from (1.10). See also Andrews [6, p. 49, Example 1].

The following two results can be used to simplify certain sums of two instances of Jacobi's triple product identity:

Quintuple Product Identity. The quintuple product identity was perhaps first stated in recognizable form by G. N. Watson [73], and independently rediscovered by W. N. Bailey [23, p. 219, eqn. (2.1)]. However, as demonstrated by Slater [69, pp. 204-205], it can be derived from a general theorem on Weierstraß's $\sigma$-functions [75, $p .451$, ex. 3]. 
Weierstraß's results on $\sigma$-functions can be found in [74].

$$
\begin{gathered}
\prod_{j=1}^{\infty}\left(1+z^{-1} q^{j}\right)\left(1+z q^{j-1}\right)\left(1-z^{-2} q^{2 j-1}\right)\left(1-z^{2} q^{2 j-1}\right)\left(1-q^{j}\right) \\
=\prod_{j=1}^{\infty}\left(1-z^{3} q^{3 j-2}\right)\left(1-z^{-3} q^{3 j-1}\right)\left(1-q^{3 j}\right)+z \prod_{j=1}^{\infty}\left(1-z^{-3} q^{3 j-2}\right)\left(1-z^{3} q^{3 j-1}\right)\left(1-q^{3 j}\right)
\end{gathered}
$$

or, in abbreviated notation,

$$
\left(z^{3} q, z^{-3} q^{2}, q^{3} ; q^{3}\right)_{\infty}+z\left(z^{-3} q, z^{3} q^{2}, q^{3} ; q^{3}\right)_{\infty}=\left(-z^{-1} q,-z, q ; q\right)_{\infty}\left(z^{-2} q, z^{2} q ; q^{2}\right)_{\infty}
$$

Next, an identity of W. N. Bailey [23, p. 220, eqn. (4.1)]:

$$
\begin{gathered}
\prod_{j=1}^{\infty}\left(1+z^{2} q^{4 j-3}\right)\left(1+z^{-2} q^{4 j-1}\right)\left(1-q^{4 j}\right)+z \prod_{j=1}^{\infty}\left(1+z^{2} q^{4 j-1}\right)\left(1+z^{-2} q^{4 j-3}\right)\left(1-q^{4 j}\right) \\
=\prod_{j=1}^{\infty}\left(1+z q^{j-1}\right)\left(1+z^{-1} q^{j}\right)\left(1-q^{j}\right)
\end{gathered}
$$

or, in abbreviated notation,

$$
\left(-z^{2} q,-z^{-2} q^{3}, q^{4} ; q^{4}\right)_{\infty}+z\left(-z^{2} q^{3},-z^{-2} q, q^{4} ; q^{4}\right)_{\infty}=\left(-z,-z^{-1} q, q ; q\right)_{\infty} .
$$

We will also require the following result:

Abel's Lemma. [75, p. 57] or [4, p. 190]. If $\lim _{n \rightarrow \infty} a_{n}=L$, then

$$
\lim _{t \rightarrow 1^{-}}(1-t) \sum_{n=0}^{\infty} a_{n} t^{n}=L .
$$

And finally, the various forms of the Heine transformation of ${ }_{2} \phi_{1}$ basic hypergeometric series are given below.

Heine's Transformations. See Gasper and Rahman [37, p. 241, eqns. (III.1), (III.2), (III.3)] For $|q|<1,|z|<1$, and $|b|<1$,

$$
\begin{aligned}
\sum_{j=0}^{\infty} \frac{(a ; q)_{j}(b ; q)_{j}}{(c ; q)_{j}(q ; q)_{j}} z^{j} & =\frac{(b, a z ; q)_{\infty}}{(c, z ; q)_{\infty}} \sum_{j=0}^{\infty} \frac{(c / b ; q)_{j}(z ; q)_{j}}{(a z ; q)_{j}(q ; q)_{j}} b^{j} \\
& =\frac{(c / b, b z ; q)_{\infty}}{(c, z ; q)_{\infty}} \sum_{j=0}^{\infty} \frac{(a b z / c ; q)_{j}(b ; q)_{j}}{(b z ; q)_{j}(q ; q)_{j}}(c / b)^{j} \\
& =\frac{(a b z / c ; q)_{\infty}}{(z ; q)_{\infty}} \sum_{j=0}^{\infty} \frac{(c / a ; q)_{j}(c / b ; q)_{j}}{(c ; q)_{j}(q ; q)_{j}}(a b z / c)^{j}
\end{aligned}
$$




\subsection{Rogers-Ramanujan Type Identities}

The Rogers-Ramanujan identities (in their analytic form) may be stated as follows:

Rogers-Ramanujan Identities - analytic. Due to L. J. Rogers, 1894. If $|q|<1$, then

$$
\sum_{j=0}^{\infty} \frac{q^{j^{2}}}{(q ; q)_{j}}=\prod_{j=0}^{\infty} \frac{1}{\left(1-q^{5 j+1}\right)\left(1-q^{5 j+4}\right)}
$$

and

$$
\sum_{j=0}^{\infty} \frac{q^{j(j+1)}}{(q ; q)_{j}}=\prod_{j=0}^{\infty} \frac{1}{\left(1-q^{5 j+2}\right)\left(1-q^{5 j+3}\right)} .
$$

The Rogers-Ramanujan identities are also of interest in combinatorics. The series and products in the above theorem are generating functions for certain classes of integer partitions. (A partition of a nonnegative integer $n$ is an unordered representation of $n$ into positive integral summands. Each summand is called a part of the partition.) Indeed, MacMahon [50, Chapter 3] realized by 1918 that the Rogers-Ramanujan identities may be stated combinatorially as follows:

First Rogers-Ramanujan Identity-combinatorial. The number of partitions of an integer $n$ into distinct, nonconsecutive parts equals the number of partitions of $n$ into parts congruent to 1 or $4(\bmod 5)$.

Second Rogers-Ramanujan Identity-combinatorial. The number of partitions of an integer $n$ into distinct, nonconsecutive parts, all of which are at least 2 equals the number of partitions of $n$ into parts congruent to 2 or $3(\bmod 5)$.

By 1980, physicist Rodney Baxter had discovered that the Rogers-Ramanujan identities were intimately linked to his solution of the hard hexagon model in statistical mechanics. His results appear in [24], [25] and [26]. The version of the Rogers-Ramanujan identities preferred by physicists is given next.

Rogers-Ramanujan Identities-fermionic/bosonic. If $|q|<1$, then

$$
\sum_{j=0}^{\infty} \frac{q^{j^{2}}}{(q ; q)_{j}}=\frac{1}{(q ; q)_{\infty}} \sum_{j=-\infty}^{\infty}\left(q^{j(10 j+1)}-q^{(5 j+2)(2 j+1)}\right)
$$

and

$$
\sum_{j=0}^{\infty} \frac{q^{j(j+1)}}{(q ; q)_{j}}=\frac{1}{(q ; q)_{\infty}} \sum_{j=-\infty}^{\infty}\left(q^{j(10 j+3)}-q^{(5 j+1)(2 j+1)}\right) .
$$

In the language of the physicists, the left hand sides of (1.65) and (1.66) are called "fermionic" representations, and the right-hand sides are called "bosonic" representations. For convenience, I will adopt this terminology, and use it througout this paper. The equality of the right-hand sides of (1.63) and (1.65) and the equality of the right-hand sides of (1.64) and (1.66) are direct consequences of (1.55) and (1.58).

Around the same time as Baxter was working on the hard hexagon model, it was discovered by Lepowski and Wilson [49] that Rogers-Ramanujan identities have a Lie theoretic interpretation and proof. 
There are many series-product identites which resemble the Rogers-Ramanujan identities in form, and are thus called "identities of the Rogers-Ramanujan type." The seminal papers in the subject from an analytic viewpoint include L. J. Rogers' papers from 1894 [57] and 1917 [58], F. H. Jackson's 1928 paper [46], and W. N. Bailey's papers of 1947 [21] and 1949 [22]. Around 1950, Lucy J. Slater, a student of W. N. Bailey, produced a list of 130 identities of the Rogers-Ramanujan type as a part of her Ph.D. thesis and published them in [68]. An annotated version of Slater's list is included as Appendix 1. Much of the early history of the Rogers-Ramanujan identities is discussed by Hardy in [43]. Andrews outlines much of the history through 1970 in [3].

The ideas (now known as the "Bailey pair," "Bailey's Lemma," and the "Bailey transform," see [10, Chapter 3]) that proved central to the discovery of large numbers of Rogers-Ramanujan type identities is due to Bailey [21] and was exploited extensively by Slater in [68]. The full iterative potential of Bailey's Lemma (dubbed "Bailey chains" by Andrews), was explored by Peter Paule in [51] and [52] and by Andrews [8].

Seminal contributions to the combinatorial aspect of Rogers-Ramanujan type identites were made by I. Schur ([64] and [65]), P. A. MacMahon [50], H. Göllnitz [40], and B. Gordon ([41] and [42]). H. L. Alder [1] provided a nice survey article of Rogers-Ramanujan history from the partition theoretic viewpoint.

Besides the contributions of Baxter listed above, other seminal contributions to the entry of the Rogers-Ramanujan identites into physics were made by Andrews, Baxter and Forrester [18] and [36], and by the Kyoto group [34]. Starting in the 1990's, Alexander Berkovich and Barry McCoy ([27] and [28]), sometimes jointly with William Orrick [30] or Anne Schilling [31], along with Ole Warnaar ([70], [71], [72]), made significant contributions to the study of Rogers-Ramanujan type identities via various models from statistical mechanics. In [29], Berkovich and McCoy present a history from the viewpoint of physics.

\section{Finitization of Rogers-Ramanujan Type Identities}

\subsection{The Method of $q$-Difference Equations}

We now turn our attention to a method for discovering finite analogs of Rogers-Ramanujan type identities via $q$-difference equations. I have automated much of the process on the computer algebra system Maple, in a package entitled "RRtools," which is documented in [67].

The method of $q$-difference equations was pioneered by Andrews in [10, $\S 9.2$, p. 88]: We begin with an identity of the Rogers-Ramanujan type

$$
\phi(q)=\Pi(q)
$$

where $\phi(q)$ is the series and $\Pi(q)$ is an infinite product or sum of several infinite products. We consider a two variable generalization $f(q, t)$ which satisfies the following three conditions: 


\section{Conditions 2.1.}

1. $f(q, t)=\sum_{n=0}^{\infty} P_{n}(q) t^{n}$ where the $P_{n}(q)$ are polynomials,

2. $\phi(q)=\lim _{t \rightarrow 1^{-}}(1-t) f(q, t)=\lim _{n \rightarrow \infty} P_{n}(q)=\Pi(q)$, and

3. $f(q, t)$ satisfies a nonhomogeneous $q$-difference equation of the form

$$
f(q, t)=R_{1}(q, t)+R_{2}(q, t) f\left(q, t q^{k}\right)
$$

where $R_{i}(q, t)$ are rational functions of $q$ and $t$ for $i=1,2$ and $k \in \mathbb{Z}_{+}$.

Theorem 2.2. If $\phi(q)$ is written in the form

$$
\sum_{j=0}^{\infty} \frac{(-1)^{a j} q^{b j^{2}+c j} \prod_{i=1}^{r}\left(d_{i} q^{e_{i}} ; q^{k_{i}}\right)_{j+l_{i}}}{\left(q^{m} ; q^{m}\right)_{j} \prod_{i=1}^{s}\left(\delta_{i} q^{\epsilon_{i}} ; q^{\kappa_{i}}\right)_{j+\lambda_{i}}}
$$

where $a=0$ or $1 ; b, m \in \mathbb{Z}_{+} ; c \in \mathbb{Z}$;

$d_{i}= \pm 1 ; e_{i}, k_{i} \in \mathbb{Z}_{+}, l_{i} \in \mathbb{Z}$ for $1 \leqq i \leqq r ;$

$\delta_{i}= \pm 1 ; \epsilon_{i}, \kappa_{i} \in \mathbb{Z}_{+} ; \lambda_{i} \in \mathbb{Z}$ for $1 \leqq i \leqq s ;$ then

$$
f(q, t)=\sum_{j=0}^{\infty} \frac{(-1)^{a j} t^{2 b j / g} q^{b j^{2}+c j} \prod_{i=1}^{r}\left(d_{i} t^{k_{i} / g} q^{e_{i}} ; q^{k_{i}}\right)_{j+l_{i}}}{\left(t ; q^{m}\right)_{j+1} \prod_{i=1}^{s}\left(\delta_{i} t^{\kappa_{i} / g} q^{\epsilon_{i}} ; q^{\kappa_{i}}\right)_{j+\lambda_{i}}}
$$

where $g=\operatorname{gcd}\left(m, k_{1}, k_{2}, \ldots, k_{r}, \kappa_{1}, \kappa_{2}, \ldots, \kappa_{s}\right)$ is a two variable generalization of $\phi(q)$ which satisfies Conditions 2.1.

Proof. First, we will demonstrate that $f(q, t)$ satisfies condition (3):

$$
\begin{aligned}
& f(q, t)=\frac{\prod_{i=1}^{r}\left(d_{i} t^{k_{i} / g} q^{e_{i}} ; q^{k_{i}}\right){ }_{l_{i}}}{(1-t) \prod_{i=1}^{s}\left(\delta_{i} t^{\kappa_{i} / g} q^{\epsilon_{i}} ; q^{\kappa_{i}}\right)_{\lambda_{i}}} \\
& +\sum_{j=1}^{\infty} \frac{(-1)^{a j} t^{2 b j / g} q^{b j^{2}+c j} \prod_{i=1}^{r}\left(d_{i} t^{k_{i} / g} q^{e_{i}} ; q^{k_{i}}\right)_{j+l_{i}}}{\left(t ; q^{m}\right)_{j+1} \prod_{i=1}^{s}\left(\delta_{i} t^{\kappa_{i} / g} q^{\epsilon_{i}} ; q^{\kappa_{i}}\right)_{j+\lambda_{i}}} \\
& =\frac{\prod_{i=1}^{r}\left(d_{i} t^{k_{i} / g} q^{e_{i}} ; q^{k_{i}}\right)_{l_{i}}}{(1-t) \prod_{i=1}^{s}\left(\delta_{i} t^{\kappa_{i} / g} q^{\epsilon_{i}} ; q^{\kappa_{i}}\right)_{\lambda_{i}}} \\
& +\sum_{j=0}^{\infty} \frac{(-1)^{a j+a} t^{2 b j+2 b / g} q^{b j^{2}+2 b j+b+c j+c} \prod_{i=1}^{r}\left(d_{i} t^{k_{i} / g} q^{e_{i}} ; q^{k_{i}}\right)_{j+l_{i}+1}}{\left(t ; q^{m}\right)_{j+2} \prod_{i=1}^{s}\left(\delta_{i} t^{\kappa_{i} / g} q^{\epsilon_{i}} ; q^{\kappa_{i}}\right)_{j+\lambda_{i}+1}} \\
& =\frac{\prod_{i=1}^{r}\left(d_{i} t^{k_{i} / g} q^{e_{i}} ; q^{k_{i}}\right)_{l_{i}}}{(1-t) \prod_{i=1}^{s}\left(\delta_{i} t^{\kappa_{i} / g} q^{\epsilon_{i}} ; q^{\kappa_{i}}\right)_{\lambda_{i}}} \\
& +\frac{(-1)^{a} t^{2 b / g} q^{b+c} \prod_{i=1}^{r}\left(1-d_{i} t^{k_{i} / g} q^{e_{i}}\right)}{(1-t) \prod_{i=1}^{s}\left(1-\delta_{i} t^{\kappa_{i} / g} q^{\epsilon_{i}}\right)}
\end{aligned}
$$




$$
\begin{gathered}
\quad \times \sum_{j=0}^{\infty} \frac{(-1)^{a j} t^{2 b j / g} q^{b j^{2}+2 b j+c j} \prod_{i=1}^{r}\left(d_{i} t^{k_{i} / g} q^{e_{i}+k_{i}} ; q^{k_{i}}\right)_{j+l_{i}}}{\left(t q^{m} ; q^{m}\right)_{j+1} \prod_{i=1}^{s}\left(\delta_{i} t^{\kappa_{i} / g} q^{\epsilon_{i}+\kappa_{i}} ; q^{\kappa_{i}}\right)_{j+\lambda_{i}}} \\
=\frac{\prod_{i=1}^{r}\left(d_{i} t^{k_{i} / g} q^{e_{i}} ; q^{k_{i}}\right)_{l_{i}}}{(1-t) \prod_{i=1}^{s}\left(\delta_{i} t^{\kappa_{i} / g} q^{\epsilon_{i}} ; q^{\kappa_{i}}\right)_{\lambda_{i}}} \\
+\frac{(-1)^{a} t^{2 b / g} q^{b+c} \prod_{i=1}^{r}\left(1-d_{i} t^{k_{i} / g} q^{e_{i}}\right)}{(1-t) \prod_{i=1}^{s}\left(1-\delta_{i} t^{\kappa_{i} / g} q^{\epsilon_{i}}\right)} f\left(q, t q^{g}\right)
\end{gathered}
$$

Now that we have an explicit formula for $f(q, t)$ and a non-homogeneous $q$-difference equation (which is first order in $q^{g}$ ) satisfied by $f(q, t)$, one can, after clearing out denominators, collect powers of $t$, and see that the coëfficient of $t^{n}$ is a polynomial $P_{n}(q)$, and thus condition (1) is satisfied.

Finally, condition (2) is satisfied as a direct consequence of (1.59).

The nonhomogeneous $q$-difference equation can be used to find a recurrence which the $P_{n}(q)$ satisfy, and thus a list of $P_{0}(q), P_{1}(q), \ldots, P_{N}(q)$ can be produced for any $N$.

The fermionic representation of the finitization is obtained by expanding the rising $q$-factorials which appear in $f(q, t)$ using (1.10) and (1.11), changing variables so that the resulting power of $t$ is $n$, so that $P_{n}(q)$ can be seen as the coëfficient of $t^{n}$.

Obtaining the bosonic representation for $P_{n}(q)$ is trickier, and requires conjecturing the correct form. Note that the RRtools Maple package contains a number of tools to aid the user in making an appropriate conjecture; see [67].

After the proposed polynomial identity is (correctly) conjectured, it can be proved by one of the techniques discussed in $\S 4$.

\subsection{A Detailed Example}

To serve as a prototypical example, we will examine the finitization process on identity (A.7) from Slater's list, an identity due to Euler:

$$
\sum_{j=0}^{\infty} \frac{q^{j^{2}+j}}{\left(q^{2} ; q^{2}\right)_{j}}=\prod_{j=1}^{\infty}\left(1+q^{2 j}\right)
$$

Due to its extreme simplicity, it is hoped that the general method will be made transparent. There are various "short cuts" which will be not be exploited since such short cuts are not applicable in more general settings.

The two variable generalization of the LHS of (2.1) is

$$
f(q, t)=\sum_{j=0}^{\infty} \frac{t^{j} q^{j^{2}+j}}{(1-t)\left(t q^{2} ; q^{2}\right)_{j}} .
$$

Next, we produce the non-homogenous $q$-difference equation. The details of the calcula- 
tion are written below.

$$
\begin{aligned}
f(q, t) & =\sum_{j=0}^{\infty} \frac{t^{j} q^{j^{2}+j}}{\left(t ; q^{2}\right)_{j+1}} \\
& =\frac{1}{1-t}+\sum_{j=1}^{\infty} \frac{t^{j} q^{j^{2}+j}}{\left(t ; q^{2}\right)_{j+1}} \\
& =\frac{1}{1-t}+\sum_{j=0}^{\infty} \frac{t^{j+1} q^{(j+1)^{2}+(j+1)}}{\left(t ; q^{2}\right)_{j+2}} \\
& =\frac{1}{1-t}+\frac{t q^{2}}{1-t} \sum_{j=0}^{\infty} \frac{\left(t q^{2}\right)^{j} q^{j^{2}+j}}{\left(t q^{2} ; q^{2}\right)_{j+1}} \\
& =\frac{1}{1-t}+\frac{t q^{2}}{1-t} f\left(q, t q^{2}\right)
\end{aligned}
$$

Thus, the non-homogeneous $q$-difference equation satisfied by $f(q, t)$ is

$$
f(q, t)=\frac{1}{1-t}+\frac{t q^{2}}{1-t} f\left(q, t q^{2}\right) .
$$

Next, we find the sequence of polynomials $\left\{P_{n}(q)\right\}_{n=0}^{\infty}$ as follows:

Clearing denominators in (2.2) gives

$$
(1-t) f(q, t)=1+t q^{2} f\left(q, t q^{2}\right)
$$

which is equivalent to

$$
f(q, t)=1+t f(q, t)+t q^{2} f\left(q, t q^{2}\right)
$$

Thus,

$$
\begin{aligned}
\sum_{n=0}^{\infty} P_{n}(q) t^{n} & =1+t \sum_{n=0}^{\infty} P_{n}(q) t^{n}+t q^{2} \sum_{n=0}^{\infty} P_{n}(q)\left(t q^{2}\right)^{n} \\
& =1+\sum_{n=0}^{\infty} P_{n}(q) t^{n+1}+\sum_{n=0}^{\infty} P_{n}(q) t^{n+1} q^{2 n+2} \\
& =1+\sum_{n=1}^{\infty} P_{n-1}(q) t^{n}+\sum_{n=1}^{\infty} q^{2 n} P_{n-1}(q) t^{n} \\
& =1+\sum_{n=1}^{\infty}\left(1+q^{2 n}\right) P_{n-1}(q) t^{n}
\end{aligned}
$$

We can read off from the last line that the polynomial sequence $\left\{P_{n}(q)\right\}_{n=0}^{\infty}$ satisfies the following recurrence relation:

$$
\begin{gathered}
P_{0}(q)=1 \\
P_{n}(q)=\left(1+q^{2 n}\right) P_{n-1}, \quad \text { if } n \geqq 1 .
\end{gathered}
$$


Note that for this example, since a first order recurrence was obtained, $P_{n}(q)$ is expressible as a finite product, and thus in some sense, the problem is done. However, the overwhelming majority of the identities from Slater's list yield finitizations whose minimimal recurrence order is greater than one, and thus not expressible as a finite product. In such cases, we must work harder to find a representation for $P_{n}(q)$ which can be seen to converge in a direct fashion to the RHS of the original identity. Thus we continue the demonstration:

Now that a recurrence for the $P_{n}(q)$ is known, a finite list $\left\{P_{n}(q)\right\}_{n=0}^{N}$ can be produced:

$$
\begin{aligned}
& P_{0}(q)=1 \\
& P_{1}(q)=q^{2}+1 \\
& P_{2}(q)=q^{6}+q^{4}+q^{2}+1 \\
& P_{3}(q)=q^{12}+q^{10}+q^{8}+2 q^{6}+q^{4}+q^{2}+1 \\
& P_{4}(q)=q^{20}+q^{18}+q^{16}+2 q^{14}+2 q^{12}+2 q^{10}+2 q^{8}+2 q^{6}+q^{4}+q^{2}+1
\end{aligned}
$$

Notice that the degree of $P_{n}(q)$ appears to be $n(n+1)$. Being familiar with Gaussian polynomials, we recall that the degree of $\left[\begin{array}{c}2 n+1 \\ n+1\end{array}\right]_{q}$ is also $n(n+1)($ by $(1.2))$, and wonder if Gaussian polynomials might play a fundamental rôle in the bosonic representation of $P_{n}(q)$. Also, since

$$
\Pi(q)=\frac{\left(q, q^{3}, q^{4} ; q^{4}\right)_{\infty}}{(q ; q)_{\infty}}
$$

(an instance of Jacobi's triple product identity multiplied by $\left.1 /(q ; q)_{\infty}\right)$ and

$$
\lim _{n \rightarrow \infty}\left[\begin{array}{c}
2 n+1 \\
n+1
\end{array}\right]_{q}=\frac{1}{(q ; q)_{\infty}} \quad(\text { by } 1.8)
$$

we have further evidence in favor of the Gaussian polynomial $\left[\begin{array}{c}2 n+1 \\ n+1\end{array}\right]_{q}$ playing a central rôle. Using the method of successive approximations by Gaussian polynomials discussed by Andrews and Baxter in $[16]^{3}$, one can conjecture that, at least for small $n$, it is true that

$$
P_{n}(q)=\left[\begin{array}{c}
2 n+1 \\
n+1
\end{array}\right]_{q}-q\left[\begin{array}{c}
2 n+1 \\
n+2
\end{array}\right]_{q}-q^{3}\left[\begin{array}{c}
2 n+1 \\
n+3
\end{array}\right]_{q}+q^{6}\left[\begin{array}{c}
2 n+1 \\
n+4
\end{array}\right]_{q}+q^{10}\left[\begin{array}{c}
2 n+1 \\
n+5
\end{array}\right]_{q}-\ldots,
$$

which is a good start, but the bosonic representation must be a bilateral series, i.e. a series where the index of summation runs over all integers, not just the nonnegative integers. Thus we employ (1.4) to rewrite the above as

$$
P_{n}(q)=\left[\begin{array}{c}
2 n+1 \\
n+1
\end{array}\right]_{q}-q\left[\begin{array}{c}
2 n+1 \\
n-1
\end{array}\right]_{q}-q^{3}\left[\begin{array}{c}
2 n+1 \\
n+3
\end{array}\right]_{q}+q^{6}\left[\begin{array}{c}
2 n+1 \\
n-3
\end{array}\right]_{q}+q^{10}\left[\begin{array}{c}
2 n+1 \\
n+5
\end{array}\right]_{q}-\ldots
$$

which is equivalent to

$$
P_{n}(q)=\sum_{j=-\infty}^{\infty}(-1)^{j} q^{2 j^{2}+j}\left[\begin{array}{c}
2 n+1 \\
n+2 j+1
\end{array}\right]_{q}
$$

\footnotetext{
${ }^{3}$ Once again, this method is implemented as a procedure in RRtools.
} 
which is in the desired (bosonic) form.

Obtaining the fermionic representation for $P_{n}(q)$ is more straightforward and does not involve any guesswork:

$$
\begin{aligned}
\sum_{n=0}^{\infty} P_{n}(q) t^{n} & =f(q, t) \\
& =\sum_{j=0}^{\infty} \frac{t^{j} q^{j^{2}+j}}{(1-t)\left(t q^{2} ; q^{2}\right)_{j}} \\
& =\sum_{j=0}^{\infty} t^{j} q^{j^{2}+j} \sum_{k=0}^{\infty}\left[\begin{array}{c}
j+k \\
k
\end{array}\right]_{q^{2}} t^{k} \text { by }(1.11) \\
& =\sum_{j=0}^{\infty} \sum_{k=0}^{\infty} t^{j+k} q^{j^{2}+j}\left[\begin{array}{c}
j+k \\
j
\end{array}\right]_{q^{2}} \text { by }(1.4) \\
& =\sum_{n=0}^{\infty} t^{n} \sum_{j=0}^{\infty} q^{j^{2}+j}\left[\begin{array}{c}
n \\
j
\end{array}\right]_{q^{2}} \quad(\text { by taking } n=j+k)
\end{aligned}
$$

By comparing coëfficients of $t^{n}$ in the extremes, we find

$$
P_{n}(q)=\sum_{j=0}^{\infty} q^{j^{2}+j}\left[\begin{array}{l}
n \\
j
\end{array}\right]_{q^{2}} .
$$

Combining (2.4) and (2.3), we obtain the conjectured polynomial identity

$$
\sum_{j=0}^{\infty} q^{j^{2}+j}\left[\begin{array}{l}
n \\
j
\end{array}\right]_{q^{2}}=\sum_{j=-\infty}^{\infty}(-1)^{j} q^{2 j^{2}+j}\left[\begin{array}{c}
2 n+1 \\
n+2 j+1
\end{array}\right]_{q}
$$

To see that (2.5) is indeed a finitization of (2.1), more calculations are needed:

$$
\begin{aligned}
& \lim _{n \rightarrow \infty} \sum_{j=0}^{\infty} q^{j^{2}+j}\left[\begin{array}{l}
n \\
j
\end{array}\right]_{q^{2}} \\
= & \lim _{n \rightarrow \infty} \sum_{j=0}^{\infty} q^{j^{2}+j} \frac{\left(q^{2} ; q^{2}\right)_{n}}{\left(q^{2} ; q^{2}\right)_{j}\left(q^{2} ; q^{2}\right)_{n-j}} \\
= & \sum_{j=0}^{\infty} \frac{q^{j^{2}+j}}{\left(q^{2} ; q^{2}\right)_{j}},
\end{aligned}
$$


and so the LHS of (2.5) converges to the LHS of (2.1).

$$
\begin{aligned}
& \lim _{n \rightarrow \infty} \sum_{j=-\infty}^{\infty}(-1)^{j} q^{2 j^{2}+j}\left[\begin{array}{c}
2 n+1 \\
n+2 j+1
\end{array}\right]_{q} \\
= & \frac{1}{(q ; q)_{\infty}} \sum_{j=-\infty}^{\infty}(-1)^{j} q^{2 j^{2}+j} \quad(\text { by }(1.8)) \\
= & \frac{1}{(q ; q)_{\infty}} \cdot\left(q, q^{3}, q^{4} ; q^{4}\right)_{\infty} \quad(\text { by } 1.55) \\
= & \prod_{j=1}^{\infty}\left(1+q^{2 j}\right),
\end{aligned}
$$

and so the RHS of (2.5) converges to the RHS of (2.1).

\subsection{Another Example}

Next, let us consider Identity (A.81) from Slater's list,

$$
\sum_{j=0}^{\infty} \frac{q^{j(j+1) / 2}}{\left(q ; q^{2}\right)_{j}(q ; q)_{j}}=\frac{\left(q, q^{6}, q^{7} ; q^{7}\right)_{\infty}\left(q^{5}, q^{9} ; q^{14}\right)_{\infty}}{(q ; q)_{\infty} /(-q ; q)_{\infty}}
$$

The two variable generalization of the LHS of (2.6) is

$$
f(q, t)=\sum_{j=0}^{\infty} \frac{t^{j} q^{j(j+1) / 2}}{\left(t^{2} q ; q^{2}\right)_{j}(t ; q)_{j+1}} .
$$

Thus, the first order non-homogeneous $q$-difference equations satisfied by $f(q, t)$ is

$$
f(q, t)=\frac{1}{1-t}+\frac{t q}{(1-t)\left(1-t^{2} q\right)} f(q, t q) .
$$

As before, we clear denominators, solve for $f(q, t)$, and read off the recurrence satisfied by the polynomials $P_{n}(q)$ where $f(q, t)=\sum_{n=0}^{\infty} P_{n}(q) t^{n}$ :

$$
\begin{gathered}
P_{0}(q)=1 \\
P_{1}(q)=q+1 \\
P_{2}(q)=q^{3}+q^{2}+q+1 \\
P_{n}(q)=\left(1+q^{n}\right) P_{n-1}+q P_{n-2}-q P_{n-3}, \quad \text { if } n \geqq 3 .
\end{gathered}
$$

Now that a recurrence for the $P_{n}(q)$ is known, a finite list $\left\{P_{n}(q)\right\}_{n=0}^{N}$ can be produced:

$$
\begin{aligned}
& P_{0}(q)=1 \\
& P_{1}(q)=q+1 \\
& P_{2}(q)=q^{3}+q^{2}+q+1 \\
& P_{3}(q)=q^{6}+q^{5}+q^{4}+2 q^{3}+2 q^{2}+q+1 \\
& P_{4}(q)=q^{10}+q^{9}+q^{8}+2 q^{7}+3 q^{6}+2 q^{5}+3 q^{4}+3 q^{3}+2 q^{2}+q+1
\end{aligned}
$$


Notice that the degree of $P_{n}(q)$ appears to be $n(n+1) / 2$, so we may be tempted to guess that the Gaussian polynomial $\left[\begin{array}{c}n+1 \\ n+1\end{array}\right]_{\sqrt{q}}$, plays the key rôle in the bosonic representation of $P_{n}(q)$, but let us look further before jumping to conclusions. Notice that the denominator of the infinite product side of $(2.6)$ contains $(q ; q)_{\infty} /(-q ; q)_{\infty}$, which is the reciprocal of the limit of the $T_{1}$ trinomial coëfficient (1.50), and not that of the proposed Gaussian polynomial. Successive approximations of $P_{n}(q)$ by the $T_{1}$ function for small $n$ leads to the conjecture that the bosonic representation of $P_{n}(q)$ is

$$
P_{n}(q)=\sum_{k=-\infty}^{\infty} q^{(21 k+1) k / 2} \mathrm{~T}_{1}(n+1,7 k ; \sqrt{q})-q^{(21 k+13) k / 2+1} \mathrm{~T}_{1}(n+1,7 k+2 ; \sqrt{q}) .
$$

As in the previous example, the fermionic representation is easily obtained from (2.7) by expanding each of the rising $q$-factorials by (1.11), and so we arrive at the conjectured identity

$$
\begin{gathered}
\sum_{j \geqq 0} \sum_{k \geqq 0} q^{j(j+1) / 2+k}\left[\begin{array}{c}
j+k-1 \\
k
\end{array}\right]_{q^{2}}\left[\begin{array}{c}
n-2 k \\
j
\end{array}\right]_{q} \\
=\sum_{k=-\infty}^{\infty} q^{(21 k+1) k / 2} \mathrm{~T}_{1}(n+1,7 k ; \sqrt{q})-q^{(21 k+13) k / 2+1} \mathrm{~T}_{1}(n+1,7 k+2 ; \sqrt{q}) .
\end{gathered}
$$

To see that the RHS of (2.9) is indeed a finitization of the RHS of (2.6), take the limit as $n \rightarrow \infty$ of the RHS of (2.9), apply (1.50), followed by Jacobi's triple product identity (1.55), and then the quintuple product identity (1.57). The analogous calculation for the LHS is straight forward.

\section{Polynomial Generalizations of the Identities in Slater's List}

\subsection{Introduction}

Listed below are finite analogs of the identities on Slater's list [68], along with recurrences satisfied by the polynomials. For easy reference the numbering scheme corresponds to that of Slater's list. In some cases, more than one bosonic representation was found, such as one that uses a $q$-binomial coëfficient and the other a $q$-trinomial coëfficient. In these instances the equations numbers are suffixed with a "b" and "t", respectively.

Many of these identities had been discovered previously. The identities related to Baxter's solution of the hard hexagon model from statistical mechanics $(3.14 \mathrm{~b}, 3.18 \mathrm{~b}$, 3.79b, 3.94b, 3.96b, 3.99b) were known to Andrews by 1981. By 1990, Andrews had bosonic $q$-trinomial representations for $(3.14 \mathrm{t}),(3.18 \mathrm{t}),(3.34 \mathrm{U})$, and $(3.36 \mathrm{U})$. A number of the identities are special cases of identities due Berkovich and McCoy, sometimes jointly with Orrick, which may be found in [27], [28], and [30]. Note that the BerkovichMcCoy identities first stated in [27] are proved in a later paper written jointly with Anne 
Schilling [31]. It is interesting to note that the bosonic forms of (3.2-b), (3.3-t), (3.11-b), (3.19), (3.28), (3.29), (3.31), (3.32), (3.33), (3.49), (3.50-b), (3.54), (3.91), (3.92), (3.93), (3.120), (3.121), (3.122), and (3.123) are specializations of bosonic forms from [27], [28], [30], or [31], where a hypothesis is violated, e.g. in $M\left(p, p^{\prime}\right)$ models, $p$ and $p^{\prime}$ must be relatively prime.

In his Ph.D. thesis [60], Santos conjectured complete bosonic forms for 36 of the identities listed here, and provided proofs for three of them. For an additional 16 of the identities, Santos provided a conjecture for the bosonic form for either even or odd values of $n$, but not both. Santos did not provide fermionic representations. Detailed references are provided with each of the previously known identities; the others are believed to be new.

\subsection{Identities}

Observation 3.1. Identity A.1 is Euler's pentagonal number theorem, which is an instance of Jacobi's Triple Product (Theorem 1.55 with $q$ replaced by $\sqrt{q}$ and $z=-q^{3 / 2}$ ). The series is bilateral in its most natural form and thus the method of $q$-difference equations is not applicable. Nevertheless, there are several known finitizations: e.g. Schur[64, eqn. (30)]. See also Paule and Riese [54, p. 22, eqn. (15)] for a different finitization due to Rogers.

Identity 3.2 (Finite forms of A.2/7). This identity is equivalent to an instance of the q-binomial theorem: eqn. (1.10) with $q$ replaced by $q^{2}$ and $t=-q^{2}$.

$$
\begin{gathered}
\sum_{j \geqq 0} q^{j^{2}+j}\left[\begin{array}{l}
n \\
j
\end{array}\right]_{q^{2}}=\sum_{j=-\infty}^{\infty}(-1)^{j} q^{2 j^{2}+j}\left[\begin{array}{c}
2 n+1 \\
n+2 j+1
\end{array}\right]_{q} \\
=\sum_{k=-\infty}^{\infty} q^{12 k^{2}+2 k} \mathrm{~T}_{1}(n+1,6 k+1 ; q)-q^{12 k^{2}+10 k+2} \mathrm{~T}_{1}(n+1,6 k+3 ; q) \\
=\left(-q^{2} ; q^{2}\right)_{n} \\
P_{0}=1, \\
P_{n}=\left(1+q^{2 n}\right) P_{n-1} \text { if } n \geqq 1 .
\end{gathered}
$$

Identity 3.3 (Finite forms of A.3/23 (with $q$ replaced by $-q$ )). Identity 3.3-b is an instance of the $q$-binomial theorem: (1.10) with $q$ replaced by $q^{2}$ and $t=-q$.

$$
\begin{gathered}
\sum_{j \geqq 0} q^{j^{2}}\left[\begin{array}{c}
n \\
j
\end{array}\right]_{q^{2}}=\sum_{j=-\infty}^{\infty}(-1)^{j} q^{2 j^{2}}\left[\begin{array}{c}
2 n \\
n+2 j
\end{array}\right]_{q} \\
=\sum_{j=-\infty}^{\infty}(-1)^{j} q^{3 j^{2}+j} \mathrm{U}(n, 3 j ; q) \\
=\left(-q ; q^{2}\right)_{n}
\end{gathered}
$$




$$
\begin{gathered}
P_{0}=1, \\
P_{n}=\left(1+q^{2 n-1}\right) P_{n-1} \text { if } n \geqq 1 .
\end{gathered}
$$

Identity 3.4 (Finite form of A.4).

$$
\begin{gathered}
\sum_{i \geqq 0} \sum_{j \geqq 0} \sum_{k \geqq 0}(-1)^{j+k} q^{i^{2}+j^{2}+2 k}\left[\begin{array}{l}
j \\
i
\end{array}\right]_{q^{2}}\left[\begin{array}{c}
j+k-1 \\
k
\end{array}\right]_{q^{2}}\left[\begin{array}{c}
n-i-k \\
j
\end{array}\right]_{q^{2}} \\
=\sum_{j=-\infty}^{\infty}(-1)^{j} q^{j^{2}} \mathrm{U}(n-1, j ; q) \\
P_{0}=1, \\
P_{1}=-q+1, \\
P_{n}=\left(1-q^{2}-q^{2 n-1}\right) P_{n-1}+\left(q^{2}-q^{2 n-2}\right) P_{n-2} \text { if } n \geqq 2 .
\end{gathered}
$$

Identity 3.5 (Finite forms of A.5/9/84). Identity 3.5-b is a special case of an identity due to Berkovich and McCoy [27, p. 59, eqn. (3.14) with $L=2 n, p=3, p^{\prime}=4, r=3 / 2$, $s=4 / 3, a=b=2$ ].

$$
\begin{gathered}
\sum_{j \geqq 0} q^{2 j^{2}+j}\left[\begin{array}{c}
n+1 \\
2 j+1
\end{array}\right]_{q}=\sum_{j=-\infty}^{\infty}(-1)^{j} q^{3 j^{2}+j}\left[\begin{array}{c}
2 n \\
n+2 j
\end{array}\right]_{q} \\
=\sum_{k=-\infty}^{\infty} q^{6 k^{2}+k} \mathrm{~T}_{1}(n+1,6 k+1 ; \sqrt{q})-\sum_{k=-\infty}^{\infty} q^{6 k^{2}+5 k+1} \mathrm{~T}_{1}(n+1,6 k+3 ; \sqrt{q}) \\
=(-q ; q)_{n} \\
P_{0}=1, \\
P_{n}=\left(1+q^{n}\right) P_{n-1} \text { if } n \geqq 1 .
\end{gathered}
$$

Identity 3.6 (Finite form of A.6).

$$
\begin{aligned}
& \sum_{i \geqq 0} \sum_{j \geqq 0} \sum_{k \geqq 0} q^{j^{2}+i(i-1) / 2+k}\left[\begin{array}{c}
j-1 \\
i
\end{array}\right]_{q}\left[\begin{array}{c}
j+k-1 \\
k
\end{array}\right]_{q^{2}}\left[\begin{array}{c}
n-j-i-2 k \\
j
\end{array}\right]_{q} \\
& = \begin{cases}\sum_{k} q^{6 k^{2}+k}\left[\begin{array}{c}
2 m \\
m+3 k
\end{array}\right]_{q}+q^{6 k^{2}+5 k+1}\left[\begin{array}{c}
2 m-1 \\
m+3 k+1
\end{array}\right]_{q} & \text { if } n=2 m, \\
\sum_{k} q^{6 k^{2}+k}\left[\begin{array}{c}
2 m \\
m+3 k
\end{array}\right]_{q}+q^{6 k^{2}+5 k+1}\left[\begin{array}{c}
2 m+1 \\
m+3 k+2
\end{array}\right]_{q} & \text { if } n=2 m+1\end{cases} \\
& P_{0}=P_{1}=1 \\
& P_{n}=P_{n-1}+\left(q+q^{n-1}\right) P_{n-2}+\left(q^{n-2}-q\right) P_{n-3} \text { if } n \geqq 3
\end{aligned}
$$

Observation 3.7. Identity (7) is (2) with $q$ replaced by $q^{2}$. 
Identity 3.8 (Finite form of A.8). Due to Berkovich, McCoy and Orrick, [30, p. 805, eqn. (2.34) with $L=n+1, \nu=2, s^{\prime}=1, r^{\prime}=0$ ]

$$
\begin{gathered}
\sum_{j \geqq 0} \sum_{k \geqq 0} q^{j(j+1) / 2+k(k+1) / 2}\left[\begin{array}{l}
j \\
k
\end{array}\right]_{q}\left[\begin{array}{c}
n-k \\
j
\end{array}\right]_{q}=\sum_{j=-\infty}^{\infty}(-1)^{j} q^{2 j^{2}+j} \mathrm{~T}_{1}(n+1,4 j+1 ; \sqrt{q}) \\
P_{0}=1 \\
P_{1}=q+1 \\
P_{n}=\left(1+q^{n}\right) P_{n-1}+q^{n} P_{n-2} \text { if } n \geqq 2 .
\end{gathered}
$$

Observation 3.9. Identity (9) is (5) with $q$ replaced by $-q$.

Identity 3.10 (Finite form of A.10/47).

$$
\begin{gathered}
\sum_{i \geqq 0} \sum_{j \geqq 0} \sum_{k \geqq 0} q^{j^{2}+i^{2}-i+k}\left[\begin{array}{l}
j \\
i
\end{array}\right]_{q^{2}}\left[\begin{array}{c}
j+k-1 \\
k
\end{array}\right]_{q^{2}}\left[\begin{array}{c}
n-i-k \\
j
\end{array}\right]_{q^{2}} \\
=\sum_{j=-\infty}^{\infty} q^{2 j^{2}+j}\left[\mathrm{~T}_{0}(n, 2 j ; q)+\mathrm{T}_{0}(n-1,2 j ; q)\right] \\
P_{0}=1 \\
P_{1}=q+1 \\
P_{n}=\left(1+q+q^{2 n-1}\right) P_{n-1}+\left(q^{2 n-3}-q\right) P_{n-2} \text { if } n \geqq 2 .
\end{gathered}
$$

Identity 3.11 (Finite forms of A.11/51/64). Bosonic q-binomial representation conjectured by Santos [60, p. 74, eqn. 6.30]. Identity 3.11-b is a special case of an identity due to Berkovich and McCoy [27, p. 59, eqn. (3.14) with $L=2 n+1, p=4, p^{\prime}=6$, $r=s=2, a=4, b=2]$.

$$
\begin{gathered}
\sum_{j \geqq 0} \sum_{k \geqq 0} q^{j^{2}+j+k^{2}}\left[\begin{array}{l}
j \\
k
\end{array}\right]_{q^{2}}\left[\begin{array}{c}
n+j-k+1 \\
2 j+1
\end{array}\right]_{q}=\sum_{j=-\infty}^{\infty}(-1)^{j} q^{6 j^{2}+2 j}\left[\begin{array}{c}
2 n+1 \\
n+3 j+1
\end{array}\right]_{q} \\
=\sum_{j=-\infty}^{\infty} q^{4 j^{2}+2 j} \mathrm{U}(n, 2 j ; q) \\
P_{0}=1 \\
P_{1}=q^{2}+q+1 \\
P_{n}=\left(1+q+q^{2 n}\right) P_{n-1}+\left(q^{2 n-1}-q\right) P_{n-2} \text { if } n \geqq 2 .
\end{gathered}
$$

Identity 3.12 (Finite form of A.12). Bosonic representation conjectured by Santos [60, p. 64, eqn. 6.2]. The identity is a special case of Berkovich, McCoy and Orrick [30, p. 
805, eqn. (2.34) with $L=n+1, \nu=2, r^{\prime}=s^{\prime}=0$ ].

$$
\begin{gathered}
\sum_{j \geqq 0} \sum_{k \geqq 0} q^{j(j+1) / 2+k(k-1) / 2}\left[\begin{array}{l}
j \\
k
\end{array}\right]_{q}\left[\begin{array}{c}
n-k \\
j
\end{array}\right]_{q}=\sum_{j=-\infty}^{\infty}(-1)^{j} q^{2 j^{2}} \mathrm{~T}_{1}(n+1,4 j+1 ; \sqrt{q}) \\
P_{0}=1 \\
P_{1}=q+1 \\
P_{n}=\left(1+q^{n}\right) P_{n-1}+q^{n-1} P_{n-2} \text { if } n \geqq 2 .
\end{gathered}
$$

Identity 3.13 (Finite form of A.13). Note: If $P_{i, n}$ represents the polynomial sequence for identity $3 . i$ for $i=8,12,13$, then

$$
\begin{aligned}
& P_{13, n}=P_{12, n-1}+P_{8, n-1}+q^{n-1} P_{12, n-2} . \\
& \sum_{j \geqq 0} \sum_{k \geqq 0} q^{j(j-1) / 2+k(k+1) / 2}\left[\begin{array}{l}
j \\
k
\end{array}\right]_{q}\left[\begin{array}{c}
n-k \\
j
\end{array}\right]_{q} \\
& =\sum_{j=-\infty}^{\infty}(-1)^{j} q^{2 j^{2}}\left(1+q^{j}\right) \mathrm{T}_{1}(n, 4 j+1 ; \sqrt{q})+(-1)^{j} q^{2 j^{2}+n-1} \mathrm{~T}_{1}(n-1,4 j+1 ; \sqrt{q}) \\
& P_{0}=1 \\
& P_{1}=2 \\
& P_{n}=\left(1+q^{n-1}\right) P_{n-1}+q^{n-1} P_{n-2} \text { if } n \geqq 2 \text {. }
\end{aligned}
$$

Identity 3.14 (Finite forms of the 2nd Rogers-Ramanujan Identity). Fermionic representation due to MacMahon [50] Bosonic q-binomial representation (14-b) due to Schur [64]. Bosonic q-trinomial representation (14-t) due to Andrews [13, p. 5. eqn. 1.12]. Identity 3.14-b is subsumed as a special case of Berkovich and McCoy [27, p. 59, eqn. (3.14) with $\left.p=2, p^{\prime}=5, r=s=1, a=4 ; b=2,3\right]$.

$$
\begin{aligned}
& \sum_{j \geqq 0} q^{j(j+1)}\left[\begin{array}{c}
n-j \\
j
\end{array}\right]_{q}=\sum_{j=-\infty}^{\infty}(-1)^{j} q^{j(5 j+3) / 2}\left[\begin{array}{c}
n+1 \\
\left\lfloor\frac{n+5 j+3}{2}\right\rfloor
\end{array}\right]_{q} \\
& =\sum_{k=-\infty}^{\infty} q^{10 k^{2}+3 k}\left(\begin{array}{c}
n+1,5 k+1 ; q \\
5 k+1
\end{array}\right)_{2}-q^{10 k^{2}+7 k+1}\left(\begin{array}{c}
n+1,5 k+2 ; q \\
5 k+2
\end{array}\right)_{2} \\
& P_{0}=P_{1}=1 \\
& P_{n}=P_{n-1}+q^{n} P_{n-2} \text { if } n \geqq 2 \text {. }
\end{aligned}
$$


Identity 3.15 (Finite form of A.15 (with $q$ replaced by $-q$ )).

$$
\begin{gathered}
\sum_{j \geqq 0} \sum_{k \geqq 0} \sum_{l \geqq 0}(-1)^{l} q^{3 j^{2}-2 j+k+2 l}\left[\begin{array}{c}
j+k-1 \\
k
\end{array}\right]_{q^{2}}\left[\begin{array}{c}
j+l-1 \\
l
\end{array}\right]_{q^{2}}\left[\begin{array}{c}
n-2 j-k-l \\
j
\end{array}\right]_{q^{2}} \\
=\sum_{j=-\infty}^{\infty}(-1)^{j} q^{10 j^{2}+3 j}\left[\begin{array}{c}
n-2 \\
\left\lfloor\frac{n+5 j-1}{2}\right\rfloor
\end{array}\right]_{q^{2}}+(-1)^{j} q^{10 j^{2}+7 j+1}\left[\begin{array}{c}
n-2 \\
\left\lfloor\frac{n+5 j}{2}\right\rfloor
\end{array}\right]_{q^{2}} \\
P_{0}=P_{1}=P_{2}=1 \\
P_{n}=\left(1+q-q^{2}\right) P_{n-1}+\left(q^{3}+q^{2}-q\right) P_{n-2}+\left(q^{2 n-5}-q^{3}\right) P_{n-3} \text { if } n \geqq 3 .
\end{gathered}
$$

Identity 3.16 (Finite form of A.16). Bosonic representation for odd $n$ conjectured by Santos [60, p. 65, eqn. 6.4]

$$
\begin{gathered}
\sum_{j \geqq 0} \sum_{k \geqq 0}(-1)^{k} q^{j^{2}+2 j+2 k}\left[\begin{array}{c}
j+k-1 \\
k
\end{array}\right]_{q^{2}}\left[\begin{array}{c}
n-k \\
j
\end{array}\right]_{q^{2}} \\
=\sum_{k=-\infty}^{\infty} q^{10 k^{2}+3 k} \mathrm{U}(n+1,5 k ; q)-q^{10 k^{2}+7 k+1} \mathrm{U}(n+1,5 k+1 ; q) \\
P_{0}=1 \\
P_{1}=q^{3}+1 \\
P_{n}=\left(1-q^{2}+q^{2 n+1}\right) P_{n-1}+q^{2} P_{n-2} \text { if } n \geqq 2 .
\end{gathered}
$$

Identity 3.17 (Finite form of A.17). Bosonic representation conjectured for odd $n$ by Santos [60, p. 65, eqn. 6.6]

$$
\begin{gathered}
\sum_{j \geqq 0} \sum_{k \geqq 0}(-1)^{k} q^{j^{2}+j+k}\left[\begin{array}{c}
j+k \\
j
\end{array}\right]_{q^{2}}\left[\begin{array}{c}
n-k \\
j
\end{array}\right]_{q^{2}}=\sum_{j=-\infty}^{\infty}(-1)^{j} q^{j(5 j+3) / 2} \mathrm{~T}_{1}\left(n+1,\left\lfloor\frac{5 j+2}{2}\right\rfloor ; q\right) \\
P_{0}=1 \\
P_{1}=q^{2}-q+1 \\
P_{n}=\left(1-q+q^{2 n}\right) P_{n-1}+q P_{n-2} \text { if } n \geqq 2 .
\end{gathered}
$$

Identity 3.18 (Finite forms of the 1st Rogers-Ramanujan Identity). Fermionic representation due to MacMahon [50]. Bosonic q-binomial representation (18-b) due to Schur [64]. Bosonic q-trinomial representation (18-t) due to Andrews [13, p. 5, eqn. 1.11]. Identity 3.18-b is subsumed as a special case of Berkovich and McCoy [27, p. 59, eqn. (3.14) with $\left.p=2, p^{\prime}=5, r=1, s=2, a=3 ; b=2,3\right]$. 


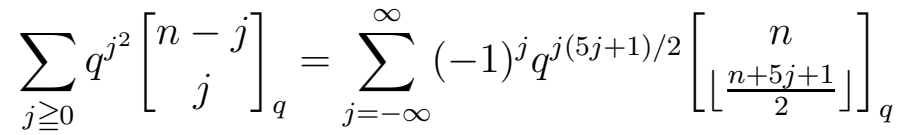

$$
\begin{aligned}
& =\sum_{k=-\infty}^{\infty} q^{10 k^{2}+k}\left(\begin{array}{c}
n, 5 k ; q \\
5 k
\end{array}\right)_{2}-q^{10 k^{2}+9 k+2}\left(\begin{array}{c}
n, 5 k+2 ; q \\
5 k+2
\end{array}\right)_{2} \\
& P_{0}=P_{1}=1 \\
& P_{n}=P_{n-1}+q^{n-1} P_{n-2} \text { if } n \geqq 2 \text {. }
\end{aligned}
$$

Identity 3.19 (Finite form of A.19). Bosonic representation conjectured for even $n$ by Santos [60, p. 66, eqn. 6.7].

$$
\begin{gathered}
\sum_{j \geqq 0} \sum_{k \geqq 0} \sum_{l \geqq 0}(-1)^{j+k+l} q^{3 j^{2}+k+2 l}\left[\begin{array}{c}
j+k-1 \\
k
\end{array}\right]_{q^{2}}\left[\begin{array}{c}
j+l-1 \\
l
\end{array}\right]_{q^{2}}\left[\begin{array}{c}
n-2 j-k-l \\
j
\end{array}\right]_{q^{2}} \\
=\sum_{j=-\infty}^{\infty}(-1)^{j} q^{j(5 j+1) / 2}\left[\begin{array}{c}
n-1 \\
\left\lfloor\frac{2 n+5}{4}\right\rfloor
\end{array}\right]_{q^{2}} \\
P_{0}=P_{1}=P_{2}=1 \\
P_{n}=\left(1-q-q^{2}\right) P_{n-1}+\left(q+q^{2}-q^{3}\right) P_{n-2}+\left(q^{3}-q^{2 n-3}\right) P_{n-3} \text { if } n \geqq 3
\end{gathered}
$$

Identity 3.20 (Finite Form of A.20). Bosonic representation due Santos [60, p. 23ff, Lemmas 2.7 and 2.8]. The proof also appears in Santos [61].

$$
\begin{gathered}
\sum_{j \geqq 0} \sum_{k \geqq 0}(-1)^{k} q^{j^{2}+2 k}\left[\begin{array}{c}
j+k-1 \\
k
\end{array}\right]_{q^{2}}\left[\begin{array}{c}
n-k \\
j
\end{array}\right]_{q^{2}} \\
=\sum_{k=-\infty}^{\infty} q^{10 k^{2}+k} \mathrm{U}(n, 5 k ; q)-q^{10 k^{2}+9 k+2} \mathrm{U}(n, 5 k+1 ; q) \\
P_{0}=1 \\
P_{1}=q+1 \\
P_{n}=\left(1-q^{2}+q^{2 n-1}\right) P_{n-1}+q^{2} P_{n-2} \text { if } n \geqq 2 .
\end{gathered}
$$

Identity 3.21 (Finite Form of A.21 (with $q$ replaced by $-q$ )).

$$
\begin{gathered}
\sum_{i \geqq 0} \sum_{j \geqq 0} \sum_{k \geqq 0} \sum_{l \geqq 0}(-1)^{l} q^{i^{2}+j^{2}+k+2 l}\left[\begin{array}{l}
j \\
i
\end{array}\right]_{q^{2}}\left[\begin{array}{c}
j+k-1 \\
k
\end{array}\right]_{q^{2}}\left[\begin{array}{c}
j+l-1 \\
l
\end{array}\right]_{q^{2}}\left[\begin{array}{c}
n-i-k-l \\
j
\end{array}\right]_{q^{2}} \\
=\sum_{j=-\infty}^{\infty}(-1)^{j} q^{10 j^{2}+j} \mathrm{U}(n, 5 j ; q)+(-1)^{j} q^{10 j^{2}+9 j+2} \mathrm{U}(n, 5 j+2 ; q) \\
P_{0}=1
\end{gathered}
$$




$$
\begin{gathered}
P_{1}=q+1 \\
P_{2}=q^{4}+2 q^{2}+q+1 \\
P_{n}=\left(1+q-q^{2}+q^{2 n-1}\right) P_{n-1}+\left(-q+q^{2}+q^{3}+q^{2 n-2}\right) P_{n-2}-q^{3} P_{n-3} \text { if } n \geqq 3 .
\end{gathered}
$$

Identity 3.22 (Finite Form of A.22). Bosonic representation conjectured by Santos [60, p. 66, eqn. 6.9]

$$
\begin{gathered}
\sum_{j \geqq 0} \sum_{k \geqq 0} \sum_{l \geqq 0} q^{j^{2}+j+k(k+1) / 2+l}\left[\begin{array}{l}
j \\
k
\end{array}\right]_{q}\left[\begin{array}{c}
j+l \\
l
\end{array}\right]_{q^{2}}\left[\begin{array}{c}
n-j-k-2 l \\
j
\end{array}\right]_{q} \\
=\sum_{j=-\infty}^{\infty}(-1)^{j} q^{3 j^{2}+2 j} \mathrm{~T}_{1}(n+1,3 j+1 ; \sqrt{q}) \\
P_{0}=P_{1}=1 \\
P_{2}=q^{2}+q+1 \\
P_{n}=P_{n-1}+\left(q+q^{n}\right) P_{n-2}+\left(q^{n}-q\right) P_{n-3} \text { if } n \geqq 3 .
\end{gathered}
$$

Observation 3.23. Identity (23) is the same as (3).

Observation 3.24. The series side of (A.24) does not contain a quadratic power of $q$, and so the method of $q$-difference equations is not applicable. Note, however, that (A.24) can be transformed to an infinite product times (A.25) via a transformation of Heine:

$$
\begin{aligned}
& \sum_{j=0}^{\infty} \frac{(-1 ; q)_{2 j} q^{j}}{\left(q^{2} ; q^{2}\right)_{j}} \\
& =\lim _{c \rightarrow 0} \sum_{j=0}^{\infty} \frac{\left(-1 ; q^{2}\right)_{j}\left(-q ; q^{2}\right)_{j} q^{j}}{\left(c ; q^{2}\right)_{j}\left(q^{2} ; q^{2}\right)_{j}} \\
& =\frac{\left(-q^{2} ; q^{2}\right)_{\infty}}{\left(q ; q^{2}\right)_{\infty}} \lim _{c \rightarrow 0} \sum_{j=0}^{\infty} \frac{\left(-q ; q^{2}\right)_{j}\left(q^{2} / c ; q^{2}\right)_{j}(c /-q)^{j}}{\left(-q^{2} ; q^{2}\right)_{j}\left(q^{2} ; q^{2}\right)_{j}} \quad \text { (by 1.61) } \\
& =\frac{\left(-q^{2} ; q^{2}\right)_{\infty}}{\left(q ; q^{2}\right)_{\infty}} \lim _{c \rightarrow 0} \sum_{j=0}^{\infty} \frac{\left(-q ; q^{2}\right)_{j}\left(1-q^{2} / c\right)\left(1-q^{4} / c\right) \ldots\left(1-q^{2 j} / c\right)(c /-q)^{j}}{\left(q^{4} ; q^{4}\right)_{j}} \\
& =\frac{\left(-q^{2} ; q^{2}\right)_{\infty}}{\left(q ; q^{2}\right)_{\infty}} \lim _{c \rightarrow 0} \sum_{j=0}^{\infty} \frac{\left(-q ; q^{2}\right)_{j}\left(c-q^{2}\right)\left(c-q^{4}\right) \ldots\left(c-q^{2 j}\right)(-q)^{-j}}{\left(q^{4} ; q^{4}\right)_{j}} \\
& =\frac{\left(-q^{2} ; q^{2}\right)_{\infty}}{\left(q ; q^{2}\right)_{\infty}} \lim _{c \rightarrow 0} \sum_{j=0}^{\infty} \frac{\left(-q ; q^{2}\right)_{j}(-1)^{j}(c-q)\left(c-q^{3}\right) \ldots\left(c-q^{2 j-1}\right)}{\left(q^{4} ; q^{4}\right)_{j}} \\
& =\frac{\left(-q^{2} ; q^{2}\right)_{\infty}}{\left(q ; q^{2}\right)_{\infty}} \sum_{j=0}^{\infty} \frac{\left(-q ; q^{2}\right)_{j}(-1)^{j}(-q)\left(-q^{3}\right) \ldots\left(-q^{2 j-1}\right)}{\left(q^{4} ; q^{4}\right)_{j}} \\
& =\frac{\left(-q^{2} ; q^{2}\right)_{\infty}}{\left(q ; q^{2}\right)_{\infty}} \sum_{j=0}^{\infty} \frac{\left(-q ; q^{2}\right)_{j} q^{j^{2}}}{\left(q^{4} ; q^{4}\right)_{j}}
\end{aligned}
$$


Also note that the infinite product representation of (A.26) is the same as that of (A.24), thus the polynomial sequence indicated in (3.26) is, in some sense, a finitization of (A.24) as well as (A.26).

Identity 3.25 (Finite Form of A.25). Bosonic representation for even n conjectured by Santos [60, p. 67, eqn. 6.11]

$$
\begin{gathered}
\sum_{i \geqq 0} \sum_{j \geqq 0} \sum_{k \geqq 0}(-1)^{k} q^{i^{2}+j^{2}+2 k}\left[\begin{array}{l}
j \\
i
\end{array}\right]_{q^{2}}\left[\begin{array}{c}
j+k-1 \\
k
\end{array}\right]_{q^{2}}\left[\begin{array}{c}
n-i-k \\
j
\end{array}\right]_{q^{2}} \\
=\sum_{j=-\infty}^{\infty}(-1)^{j} q^{3 j^{2}} \mathrm{U}(n, 3 j ; q) \\
P_{0}=1 \\
P_{1}=q+1 \\
P_{n}=\left(1-q^{2}+q^{2 n-1}\right) P_{n-1}+\left(q^{2}+q^{2 n-2}\right) P_{n-2} \text { if } n \geqq 2 .
\end{gathered}
$$

Identity 3.26 (Finite Form of A.26). Bosonic representation conjectured by Santos [60, p. 67, eqn. 6.12].

$$
\begin{gathered}
\sum_{j \geqq 0} \sum_{k \geqq 0} \sum_{l \geqq 0} q^{j^{2}+k(k+1) / 2+l}\left[\begin{array}{l}
j \\
k
\end{array}\right]_{q}\left[\begin{array}{c}
j+l \\
l
\end{array}\right]_{q^{2}}\left[\begin{array}{c}
n-j-k-2 l \\
j
\end{array}\right]_{q} \\
=\sum_{j=-\infty}^{\infty}(-1)^{j} q^{3 j^{2}} \mathrm{~T}_{1}(n, 3 j ; \sqrt{q}) \\
P_{0}=P_{1}=1 \\
P_{2}=2 q+1 \\
P_{n}=P_{n-1}+\left(q+q^{n-1}\right) P_{n-2}+\left(q^{n-1}-q\right) P_{n-3} \text { if } n \geqq 3 .
\end{gathered}
$$

Identity 3.27 (Finite Form of A.27/87). Bosonic representation for even $n$ conjectured by Santos [60, p. 82, eqn. 6.50]. Bosonic representation for odd $n$ conjectured by Santos [60, p. 68, eqn. 6.13].

$$
\begin{aligned}
& \sum_{i \geqq 0} \sum_{j \geqq 0} \sum_{k \geqq 0} \sum_{l \geqq 0}(-1)^{l} q^{2 j^{2}+2 j+i^{2}+k+2 l}\left[\begin{array}{l}
j \\
i
\end{array}\right]_{q^{2}}\left[\begin{array}{c}
j+k \\
k
\end{array}\right]_{q^{2}}\left[\begin{array}{c}
j+l-1 \\
l
\end{array}\right]_{q^{2}}\left[\begin{array}{c}
n-j-i-k-l \\
j
\end{array}\right]_{q^{2}}
\end{aligned}
$$

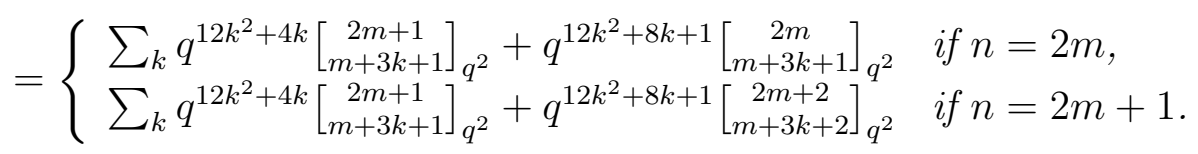

$$
\begin{aligned}
& P_{0}=1 \\
& P_{1}=q+1 \\
& P_{2}=q^{4}+q^{2}+q+1 \\
& P_{n}=\left(1+q-q^{2}\right) P_{n-1}+\left(q^{2 n}+q^{3}+q^{2}-q\right) P_{n-2}+\left(q^{2 n-1}-q^{3}\right) P_{n-3} \text { if } n \geqq 3
\end{aligned}
$$


Identity 3.28 (Finite form of A.28). Bosonic representation for even $n$ conjectured by Santos [60, p. 68, eqn. 6.14].

$$
\begin{gathered}
\sum_{j \geqq 0} \sum_{k \geqq 0} q^{j^{2}+j+k^{2}+k}\left[\begin{array}{l}
j \\
k
\end{array}\right]_{q^{2}}\left[\begin{array}{c}
n+j-k+1 \\
2 j+1
\end{array}\right]_{q}=\sum_{j=-\infty}^{\infty} q^{3 j^{2}+2 j} \mathrm{~T}_{1}(n+1,3 j+1 ; q) \\
P_{0}=1 \\
P_{1}=q^{2}+q+1 \\
P_{n}=\left(1+q+q^{2 n}\right) P_{n-1}+\left(q^{2 n}-q\right) P_{n-2} \text { if } n \geqq 2 .
\end{gathered}
$$

Identity 3.29 (Finite form of A.29). Bosonic representation proved by Santos [60, $p$. 17, Lemma 2.3].

$$
\begin{gathered}
\sum_{j \geqq 0} \sum_{k \geqq 0} q^{k^{2}+j^{2}}\left[\begin{array}{l}
j \\
k
\end{array}\right]_{q^{2}}\left[\begin{array}{c}
n+j-k \\
2 j
\end{array}\right]_{q}=\sum_{j=-\infty}^{\infty} q^{3 j^{2}+j} \mathrm{U}(n, 3 j ; q) \\
P_{0}=1 \\
P_{1}=q+1 \\
P_{n}=\left(1+q+q^{2 n-1}\right) P_{n-1}+\left(q^{2 n-2}-q\right) P_{n-2} \text { if } n \geqq 2 .
\end{gathered}
$$

Observation 3.30. Identity (30) is (24) with $q$ replaced by $-q$.

Identity 3.31 (Finite form of the third Rogers-Selberg Identity). Bosonic representation for even $n$ conjectured by Santos [60, p. 69, eqn. 6.16].

$$
\begin{aligned}
& \sum_{j \geqq 0} \sum_{k \geqq 0}(-1)^{k} q^{2 j^{2}+2 j+k}\left[\begin{array}{c}
2 j+k \\
k
\end{array}\right]_{q}\left[\begin{array}{c}
n-j-k \\
j
\end{array}\right]_{q^{2}}=\sum_{j=-\infty}^{\infty}(-1)^{j} q^{j(7 j+5) / 2}\left[\begin{array}{c}
n+1 \\
\left\lfloor\frac{2 n+7 j+6}{4}\right\rfloor
\end{array}\right]_{q^{2}} \\
& P_{0}=1 \\
& P_{1}=-q+1 \\
& P_{2}=q^{4}+q^{2}-q+1 \\
& P_{n}=\left(1-q-q^{2}\right) P_{n-1}+\left(q^{2 n}-q^{3}+q^{2}+q\right) P_{n-2}+q^{3} P_{n-3} \text { if } n \geqq 3 \text {. }
\end{aligned}
$$

Identity 3.32 (Finite form of second Rogers-Selberg Identity). Bosonic representation conjectured for even $n$ by Santos [60, p. 69, eqn. 6.17].

$$
\begin{gathered}
\sum_{j \geqq 0} \sum_{k \geqq 0}(-1)^{k} q^{2 j^{2}+2 j+k}\left[\begin{array}{c}
2 j+k-1 \\
k
\end{array}\right]_{q}\left[\begin{array}{c}
n-j-k \\
j
\end{array}\right]_{q^{2}} \\
=\sum_{j=-\infty}^{\infty}(-1)^{j} q^{j(7 j+3) / 2}\left[\begin{array}{c}
n+1 \\
\left\lfloor\frac{2 n+7 j+5}{4}\right\rfloor
\end{array}\right]_{q^{2}} \\
P_{0}=P_{1}=1 \\
P_{2}=q^{4}+1 \\
P_{n}=\left(1-q-q^{2}\right) P_{n-1}+\left(q^{2 n}-q^{3}+q^{2}+q\right) P_{n-2}+q^{3} P_{n-3} \text { if } n \geqq 3 .
\end{gathered}
$$


Identity 3.33 (Finite Form of the first Rogers-Selberg Identity). Bosonic representation conjectured for even $n$ by Santos [60, p. 70, eqn. 6.18].

$$
\begin{gathered}
\sum_{j \geqq 0} \sum_{k \geqq 0}(-1)^{k} q^{2 j^{2}+k}\left[\begin{array}{c}
2 j+k-1 \\
k
\end{array}\right]_{q}\left[\begin{array}{c}
n-j-k \\
j
\end{array}\right]_{q^{2}}=\sum_{j=-\infty}^{\infty}(-1)^{j} q^{j(7 j+1) / 2}\left[\begin{array}{c}
n \\
\left\lfloor\frac{2 n+7 j+2}{4}\right\rfloor
\end{array}\right]_{q^{2}} \\
P_{0}=P_{1}=1 \\
P_{2}=q^{2}+1 \\
P_{n}=\left(1-q-q^{2}\right) P_{n-1}+\left(q^{2 n-2}-q^{3}+q^{2}+q\right) P_{n-2}+q^{3} P_{n-3} \text { if } n \geqq 3 .
\end{gathered}
$$

Identity 3.34 (Finite form of the second Göllnitz-Gordon identity). Bosonic $U$ representation given by Andrews [13, p. 13, eqn. 4.10]. Fermionic/U identity given by Berkovich and McCoy [28, p. 42, eqn 2.23, with $L=n+1, \nu=2, s^{\prime}=1, r^{\prime}=0$, and $q$ replaced by $\left.q^{2}.\right]$.

$$
\begin{gathered}
\sum_{j \geqq 0} \sum_{k \geqq 0} q^{j^{2}+2 j+k^{2}}\left[\begin{array}{l}
j \\
k
\end{array}\right]_{q^{2}}\left[\begin{array}{c}
n-k \\
j
\end{array}\right]_{q^{2}}=\sum_{j=-\infty}^{\infty}(-1)^{j} q^{4 j^{2}+3 j} \mathrm{U}(n+1,4 j+1 ; q) \\
=\sum_{j=-\infty}^{\infty}(-1)^{j} q^{12 j^{2}+5 j}\left(\begin{array}{c}
n+1,4 j+1 ; q^{2} \\
4 j+1
\end{array}\right)_{2}+(-1)^{j} q^{12 j^{2}+11 j+2}\left(\begin{array}{c}
n+1,4 j+2 ; q^{2} \\
4 j+2
\end{array}\right)_{2} \\
P_{0}=1 \\
P_{1}=q^{3}+1 \\
P_{n}=\left(1+q^{2 n}\right) P_{n-1}+q^{2 n} P_{n-2} \text { if } n \geqq 2 .
\end{gathered}
$$

Identity 3.35 (Finite form of A.35/106).

$$
\begin{gathered}
\sum_{i \geqq 0} \sum_{j \geqq 0} \sum_{k \geqq 0} q^{j(j+3) / 2+i^{2}+k}\left[\begin{array}{l}
j \\
i
\end{array}\right]_{q^{2}}\left[\begin{array}{c}
j+k \\
k
\end{array}\right]_{q^{2}}\left[\begin{array}{c}
n-2 i-2 k \\
j
\end{array}\right]_{q} \\
=\sum_{j=-\infty}^{\infty}(-1)^{j} q^{4 j^{2}+3 j} \mathrm{~V}(n+2,4 j+2 ; \sqrt{q}) \\
P_{0}=1 \\
P_{1}=q^{2}+1 \\
P_{2}=q^{5}+q^{3}+q^{2}+q+1 \\
P_{n}=\left(1+q^{n+1}\right) P_{n-1}+q P_{n-2}+\left(q^{n}-q\right) P_{n-3}
\end{gathered}
$$

Identity 3.36 (Finite form of the first Göllnitz-Gordon identity). Bosonic $U$ representation given by Andrews [13, p. 11, eqn. 4.4]. Fermionic/U identity given by 
Berkovich and McCoy [28, p. 42, eqn. 2.23, with $L=n, \nu=2, s^{\prime}=r^{\prime}=0$ and $q$ replaced by $\left.q^{2}\right]$.

$$
\begin{gathered}
\sum_{j \geqq 0} \sum_{k \geqq 0} q^{j^{2}+k^{2}}\left[\begin{array}{l}
j \\
k
\end{array}\right]_{q^{2}}\left[\begin{array}{c}
n-k \\
j
\end{array}\right]_{q^{2}}=\sum_{j=-\infty}^{\infty}(-1)^{j} q^{4 j^{2}+j} \mathrm{U}(n, 4 j ; q) \\
=\sum_{j=-\infty}^{\infty}(-1)^{j} q^{12 j^{2}-j}\left(\begin{array}{c}
n, 4 j ; q^{2} \\
4 j
\end{array}\right)_{2}+(-1)^{j} q^{12 j^{2}+7 j+1}\left(\begin{array}{c}
n, 4 j+1 ; q^{2} \\
4 j+1
\end{array}\right)_{2} \\
P_{0}=1 \\
P_{1}=q+1 \\
P_{n}=\left(1+q^{2 n-1}\right) P_{n-1}+q^{2 n-2} P_{n-2} \text { if } n \geqq 2 .
\end{gathered}
$$

Identity 3.37 (Finite form of A.37/105).

$$
\begin{gathered}
\sum_{i \geqq 0} \sum_{j \geqq 0} \sum_{k \geqq 0} q^{j(j+1) / 2+i^{2}+k}\left[\begin{array}{l}
j \\
i
\end{array}\right]_{q^{2}}\left[\begin{array}{c}
j+k \\
k
\end{array}\right]_{q^{2}}\left[\begin{array}{c}
n-2 i-2 k \\
j
\end{array}\right]_{q} \\
=\sum_{j=-\infty}^{\infty}(-1)^{j} q^{4 j^{2}+j} \mathrm{~V}(n+1,4 j+1 ; \sqrt{q}) \\
P_{0}=1 \\
P_{1}=q+1 \\
P_{2}=q^{3}+q^{2}+2 q+1 \\
P_{n}=\left(1+q^{n}\right) P_{n-1}+q P_{n-2}+\left(q^{n-1}-q\right) P_{n-3} \text { if } n \geqq 3 .
\end{gathered}
$$

Identity 3.38 (Finite form of A.38/86). Bosonic representation (3.38-b) due to Santos [60]. Identity (3.38-b) stated by Andrews and Santos [20, p. 94, eqn 3.2]. Identity (3.38-t) due to Andrews [12, p. 663, eqn. (5.3)].

$$
\begin{aligned}
& \sum_{j \geqq 0} q^{2 j(j+1)}\left[\begin{array}{c}
n+1 \\
2 j+1
\end{array}\right]_{q}=\sum_{j=-\infty}^{\infty} q^{4 j^{2}+3 j}\left[\begin{array}{c}
n+1 \\
\left\lfloor\frac{n+4 j+3}{2}\right\rfloor
\end{array}\right]_{q^{2}} \\
& =\sum_{k=-\infty}^{\infty} q^{12 k^{2}+5 k}\left(\begin{array}{c}
n+1,6 k+1 ; q \\
6 k+1
\end{array}\right)_{2}-q^{12 k^{2}+11 k+2}\left(\begin{array}{c}
n+1,6 k+3 ; q \\
6 k+3
\end{array}\right)_{2} \\
& P_{0}=1 \\
& P_{1}=q+1 \\
& P_{n}=(1+q) P_{n-1}+\left(q^{2 n}-q\right) P_{n-2} \text { if } n \geqq 2 \text {. }
\end{aligned}
$$

Identity 3.39 (Finite Form of A.39/83). Bosonic representation (3.39-b) conjectured by Santos [60, p. 71, eqn. 6.22]. Identity (3.39-b) stated by Andrews and Santos [20, p. 
94, eqn. 3.1]. Identity (3.39-t) due to Andrews [12, p. 663, eqn. (5.2)].

$$
\begin{aligned}
& \sum_{j \geqq 0} q^{2 j^{2}}\left[\begin{array}{c}
n \\
2 j
\end{array}\right]_{q}=\sum_{j=-\infty}^{\infty} q^{4 j^{2}+j}\left[\begin{array}{c}
n \\
\left\lfloor\frac{n+4 j+1}{2}\right\rfloor
\end{array}\right]_{q^{2}} \\
& =\sum_{k=-\infty}^{\infty} q^{12 k^{2}+k}\left(\begin{array}{c}
n, 6 k ; q \\
6 k
\end{array}\right)_{2}-\sum_{k=-\infty}^{\infty} q^{12 k^{2}+7 k+1}\left(\begin{array}{c}
n, 6 k+2 ; q \\
6 k+2
\end{array}\right)_{2} \\
& P_{0}=P_{1}=1 \\
& P_{n}=(1+q) P_{n-1}+\left(q^{2 n-2}-q\right) P_{n-2} \text { if } n \geqq 2 \text {. }
\end{aligned}
$$

Identity 3.40 (Finite Form of A.40).

$$
\begin{gathered}
\sum_{i \geqq 0} \sum_{I \geqq 0} \sum_{j \geqq 0} \sum_{k \geqq 0} \sum_{K \geqq 0}(-1)^{i+I+K} q^{3 j^{2}+3 j+i(3 i-1) / 2+I(3 I+1) / 2+3 k+3 K}\left[\begin{array}{c}
j+1 \\
i
\end{array}\right]_{q^{3}}\left[\begin{array}{l}
j \\
I
\end{array}\right]_{q^{3}} \\
\times\left[\begin{array}{c}
j+k \\
k
\end{array}\right]_{q^{6}}\left[\begin{array}{c}
j+K-1 \\
K
\end{array}\right]_{q^{3}}\left[\begin{array}{c}
n-j-i-I-2 k-K \\
j
\end{array}\right]_{q^{3}} \\
\quad=\left\{\begin{array}{c}
\sum_{k} q^{18 j^{2}+7 j}\left[\begin{array}{c}
2 m+1 \\
m+3 j+1
\end{array}\right]_{q^{3}}-q^{18 j^{2}+11 j+1}\left[\begin{array}{c}
2 m \\
m+3 j+1
\end{array}\right]_{q^{3}} \quad \text { if } n=2 m, \\
\sum_{k} q^{18 j^{2}+7 j}\left[\begin{array}{c}
2 m+1 \\
m+3 j+1
\end{array}\right]_{q^{3}}-q^{18 j^{2}+11 j+1}\left[\begin{array}{c}
2 m+2 \\
m+3 j+2
\end{array}\right]_{q^{3}} \quad \text { if } n=2 m+1 . \\
P_{0}=1 \\
P_{2}=q^{6}+q^{3}-q+1 \\
P_{3}=-q^{10}-q^{8}-q^{7}+q^{6}-q^{4}+q^{3}-q+1 \\
+\left(q^{3 n-3}-q^{6}\right) P_{n-4} \text { if } n \geqq 4
\end{array}\right.
\end{gathered}
$$

Identity 3.41 (Finite Form of A.41).

$$
\begin{aligned}
& \sum_{i \geqq 0} \sum_{I \geqq 0} \sum_{j \geqq 0} \sum_{k \geqq 0} \sum_{K \geqq 0}(-1)^{i+I+K} q^{3 j^{2}+3 j+i(3 i-1) / 2+I(3 I+1) / 2+3 k+3 K}\left[\begin{array}{l}
j \\
i
\end{array}\right]_{q^{3}}\left[\begin{array}{c}
j+1 \\
I
\end{array}\right]_{q^{3}} \\
& \times\left[\begin{array}{c}
j+k \\
k
\end{array}\right]_{q^{6}}\left[\begin{array}{c}
j+K-1 \\
K
\end{array}\right]_{q^{3}}\left[\begin{array}{c}
n-j-i-I-2 k-K \\
j
\end{array}\right]_{q^{3}} \\
& = \begin{cases}\sum_{k} q^{18 k^{2}+5 k}\left[\begin{array}{c}
2 m+1 \\
m+3 k+1
\end{array}\right]_{q^{3}}-q^{18 k^{2}+13 k+2}\left[\begin{array}{c}
2 m \\
m+3 k+1
\end{array}\right]_{q^{3}} & \text { if } n=2 m, \\
\sum_{k} q^{18 k^{2}+5 k}\left[\begin{array}{c}
2 m+1 \\
m+3 k+1
\end{array}\right]_{q^{3}}-q^{18 k^{2}+13 k+2}\left[\begin{array}{c}
2 m+2 \\
m+3 k+2
\end{array}\right]_{q^{3}} & \text { if } n=2 m+1 .\end{cases}
\end{aligned}
$$




$$
\begin{gathered}
P_{0}=1 \\
P_{1}=-q^{2}+1 \\
P_{2}=q^{6}+q^{3}-q^{2}+1 \\
P_{3}=-q^{11}-q^{8}-q^{7}+q^{6}-q^{5}+q^{3}-q^{2}+1 \\
P_{n}=\left(1-q^{3}\right) P_{n-1}+\left(q^{3 n}+2 q^{3}\right) P_{n-2}+\left(q^{6}-q^{3}-q^{3 n-1}-q^{3 n-2}\right) P_{n-3} \\
+\left(q^{3 n-3}-q^{6}\right) P_{n-4} \text { if } n \geqq 4
\end{gathered}
$$

\section{Identity 3.42 (Finite Form of A.42).}

$$
\begin{aligned}
& \sum_{i \geqq 0} \sum_{I \geqq 0} \sum_{j \geqq 0} \sum_{k \geqq 0} \sum_{K \geqq 0}(-1)^{i+I+K} q^{3 j^{2}+i(3 i-1) / 2+I(3 I+1) / 2+3 k+3 K}\left[\begin{array}{l}
j \\
i
\end{array}\right]_{q^{3}}\left[\begin{array}{l}
j \\
I
\end{array}\right]_{q^{3}} \\
& \times\left[\begin{array}{c}
j+k-1 \\
k
\end{array}\right]_{q^{6}}\left[\begin{array}{c}
j+K-1 \\
K
\end{array}\right]_{q^{3}}\left[\begin{array}{c}
n-j-i-I-2 k-K \\
j
\end{array}\right]_{q^{3}} \\
& = \begin{cases}\sum_{k} q^{18 k^{2}+k}\left[\begin{array}{c}
2 m \\
m+3 k
\end{array}\right]_{q^{3}}-q^{18 k^{2}+17 k+4}\left[\begin{array}{c}
2 m-1 \\
m+3 k+1
\end{array}\right]_{q^{3}} & \text { if } n=2 m, \\
\sum_{k} q^{18 k^{2}+k}\left[\begin{array}{c}
2 m \\
m+3 k
\end{array}\right]_{q^{3}}-q^{18 k^{2}+17 k+4}\left[\begin{array}{c}
2 m+1 \\
m+3 k+2
\end{array}\right]_{q^{3}} & \text { if } n=2 m+1 .\end{cases} \\
& P_{0}=P_{1}=1 \\
& P_{2}=q^{3}+1 \\
& P_{3}=-q^{5}-q^{4}+q^{3}+1 \\
& P_{n}=\left(1-q^{3}\right) P_{n-1}+\left(q^{3 n}+2 q^{3}\right) P_{n-2}+\left(q^{6}-q^{3}-q^{3 n-4}-q^{3 n-5}\right) P_{n-3} \\
& +\left(q^{3 n-6}-q^{6}\right) P_{n-4} \text { if } n \geqq 4
\end{aligned}
$$

Identity 3.43 (Finite Form of A.43). Bosonic form for odd $n$ conjectured by Santos [60, p. 71, eqn. 6.23].

$$
\begin{gathered}
\sum_{j \geqq 0} \sum_{k \geqq 0} \sum_{l \geqq 0} q^{\left(j^{2}+3 j+k^{2}+k+2 l\right) / 2}\left[\begin{array}{l}
j \\
k
\end{array}\right]_{q}\left[\begin{array}{c}
j+l \\
l
\end{array}\right]_{q^{2}}\left[\begin{array}{c}
n-k-2 l \\
j
\end{array}\right]_{q} \\
=\sum_{j=-\infty}^{\infty}(-1)^{j} q^{5 j^{2}+4 j} \mathrm{~T}_{1}(n+2,5 j+2 ; \sqrt{q}) \\
P_{0}=1 \\
P_{1}=q^{2}+1 \\
P_{2}=q^{5}+2 q^{3}+q^{2}+q+1 \\
P_{n}=\left(1+q^{n+1}\right) P_{n-1}+\left(q+q^{n+1}\right) P_{n-2}-q P_{n-3} \text { if } n \geqq 3
\end{gathered}
$$

Identity 3.44 (Finite form of A.44/63). Identity (3.44-b) due to Andrews [7, p. 5291, eqn. 3.2]; corrected here. Bosonic q-trinomial representation conjectured by Santos [60, 
p. 77 , eqn. 6.38].

$$
\begin{gathered}
\sum_{j \geqq 0} \sum_{k \geqq 0} q^{3 j(j+1) / 2+k}\left[\begin{array}{c}
j+k \\
k
\end{array}\right]_{q^{2}}\left[\begin{array}{c}
n-2 j-2 k-1 \\
j
\end{array}\right]_{q}=\sum_{j=-\infty}^{\infty}(-1)^{j} q^{5 j^{2}+3 j}\left[\begin{array}{c}
n+1 \\
\left\lfloor\frac{n+5 j+3}{2}\right\rfloor
\end{array}\right]_{q} \\
=\sum_{k=-\infty}^{\infty} q^{\left(15 k^{2}+7 k\right) / 2} \mathrm{~T}_{1}(n+1,5 k+1 ; \sqrt{q})-q^{(15 k+13) k / 2+1} \mathrm{~T}_{1}(n+1,5 k+2 ; \sqrt{q}) \\
P_{0}=P_{1}=1 \\
P_{2}=q+1 \\
P_{n}=P_{n-1}+q P_{n-2}+\left(q^{n}-q\right) P_{n-3} \text { if } n \geqq 3
\end{gathered}
$$

Identity 3.45 (Finite form of A.45). Bosonic representation for even $n$ conjectured by Santos [60, p. 72, eqn 6.25].

$$
\begin{gathered}
\sum_{j \geqq 0} \sum_{k \geqq 0} \sum_{l \geqq 0} q^{\left(j^{2}+j+k^{2}+k+2 l\right) / 2}\left[\begin{array}{l}
j \\
k
\end{array}\right]_{q}\left[\begin{array}{c}
j+l \\
l
\end{array}\right]_{q^{2}}\left[\begin{array}{c}
n-k-2 l]_{q} \\
j
\end{array}\right]_{q} \sum_{j=-\infty}^{\infty}(-1)^{j} q^{5 j^{2}+2 j} \mathrm{~T}_{1}(n+1,5 j+1 ; \sqrt{q}) \\
P_{0}=1 \\
P_{1}=q+1 \\
P_{2}=q^{3}+2 q^{2}+2 q+1 \\
P_{n}=\left(1+q^{n}\right) P_{n-1}+\left(q+q^{n}\right) P_{n-2}-q P_{n-3} \text { if } n \geqq 3
\end{gathered}
$$

Identity 3.46 (Finite form of A.46/62). Identity (3.46-b) due to Andrews [7, p. 5291, eqn. 3.1]. Bosonic q-trinomial representation conjectured by Santos [60, p. 77, eqn. 6.37].

$$
\begin{gathered}
\sum_{j \geqq 0} \sum_{k \geqq 0} q^{j(3 j+1) / 2+k}\left[\begin{array}{c}
j+k \\
k
\end{array}\right]_{q^{2}}\left[\begin{array}{c}
n-2 j-2 k \\
j
\end{array}\right]_{q}=\sum_{j=-\infty}^{\infty}(-1)^{j} q^{j(5 j+1)}\left[\begin{array}{c}
n \\
\left\lfloor\frac{n-5 j}{2}\right\rfloor
\end{array}\right]_{q} \\
=\sum_{k=-\infty}^{\infty} q^{\left(15 k^{2}+k\right) / 2} \mathrm{~T}_{1}(n, 5 k ; \sqrt{q})-q^{(15 k+11) k / 2+1} \mathrm{~T}_{1}(n, 5 k+2 ; \sqrt{q}) \\
P_{0}=P_{1}=P_{2}=1 \\
P_{n}=P_{n-1}+q P_{n-2}+\left(q^{n-2}-q\right) P_{n-3} \text { if } n \geqq 3
\end{gathered}
$$

Observation 3.47. Identity (47) equivalent to (10) and to (54) $+q \times(49)$.

Observation 3.48. Identity (48) is (54) $-q \times(49)$. 


\section{Identity 3.49 (Finite form of A.49).}

$$
\begin{gathered}
\sum_{i \geqq 0} \sum_{I \geqq 0} \sum_{j \geqq 0} \sum_{k \geqq 0} \sum_{K \geqq 0}(-1)^{K} q^{j^{2}+2 j+i^{2}+i+I^{2} / 2+I / 2+k+2 K}\left[\begin{array}{l}
j \\
i
\end{array}\right]_{q^{2}}\left[\begin{array}{c}
j+1 \\
I
\end{array}\right]_{q}\left[\begin{array}{c}
j+k \\
k
\end{array}\right]_{q^{2}} \\
\times\left[\begin{array}{c}
j+K \\
K
\end{array}\right]_{q^{2}}\left[\begin{array}{c}
n-j-2 i-I-2 k-2 K \\
j
\end{array}\right]_{q} \\
=\sum_{j=-\infty}^{\infty}(-1)^{j} q^{6 j^{2}+5 j}\left[\begin{array}{c}
n+2 \\
\left\lfloor\frac{n+6 j+5}{2}\right\rfloor
\end{array}\right]_{q} \\
P_{0}=1 \\
P_{1}=-q+1 \\
P_{2}=q^{3}+q^{2}+1 \\
P_{3}=-q^{5}+1 \\
P_{n}=P_{n-1}+\left(q+q^{2}+q^{n+1}\right) P_{n-2}+\left(-q-q^{2}-q^{n+1}\right) P_{n-3}+\left(-q^{3}+q^{n+1}\right) P_{n-4} \\
+\left(q^{3}-q^{n+1}\right) P_{n-5} \text { if } n \geqq 5
\end{gathered}
$$

Identity 3.50 (Finite form of A.50). Bosonic representation (3.50-b) conjectured by Santos [60, p. 73, eqn. 6.29].

$$
\begin{gathered}
\sum_{j \geqq 0} \sum_{k \geqq 0} q^{j^{2}+2 j+k^{2}}\left[\begin{array}{l}
j \\
k
\end{array}\right]_{q^{2}}\left[\begin{array}{c}
n-j-k+1 \\
2 j+1
\end{array}\right]_{q}=\sum_{j=-\infty}^{\infty}(-1)^{j} q^{6 j^{2}+4 j}\left[\begin{array}{c}
2 n+2 \\
n+3 j+2
\end{array}\right]_{q} \\
=\sum_{j=-\infty}^{\infty} q^{12 j^{2}+6 j} \mathrm{U}(n+1,6 j+1 ; q) \\
P_{0}=1 \\
P_{1}=q^{3}+q+1 \\
P_{n}=\left(1+q+q^{2 n+1}\right) P_{n-1}+\left(q^{2 n}-q\right) P_{n-2} \text { if } n \geqq 2 .
\end{gathered}
$$

Observation 3.51. Identity (51) is the same as (11) and (64).

Identity 3.52 (Finite form of A.52).

$$
\begin{gathered}
\sum_{j \geqq 0} q^{2 j^{2}-j}\left[\begin{array}{c}
n \\
2 j
\end{array}\right]_{q}=\sum_{k=-\infty}^{\infty} q^{4 k^{2}+k}\left[\begin{array}{c}
2 n \\
n+4 k
\end{array}\right]_{\sqrt{q}}-q^{4 k^{2}+5 k+3 / 2}\left[\begin{array}{c}
2 n \\
n+4 k+3
\end{array}\right]_{\sqrt{q}} \\
=\sum_{k=-\infty}^{\infty} q^{6 k^{2}+k} \mathrm{~T}_{1}(n, 6 k ; \sqrt{q})-q^{6 k^{2}+5 k+1} \mathrm{~T}_{1}(n, 6 k+2 ; \sqrt{q}) \\
=\sum_{j=-\infty}^{\infty}(-1)^{j} q^{6 j^{2}+2 j} \mathrm{U}(n-1,3 j ; q)
\end{gathered}
$$




$$
\begin{gathered}
=(-q ; q)_{n-1} \text { if } n \geqq 2 . \\
P_{0}=P_{1}=1 \\
P_{n}=\left(1+q^{n-1}\right) P_{n-1} \text { if } n \geqq 2
\end{gathered}
$$

Identity 3.53 (Finite form of A.53). Bosonic representation for even $n$ conjectured by Santos [60, p. 74, eqn. 6.32].

$$
\begin{aligned}
& \sum_{i \geqq 0} \sum_{j \geqq 0} \sum_{k \geqq 0} \sum_{l \geqq 0}(-1)^{i+l} q^{4 j^{2}+i^{2}+4 k+4 l}\left[\begin{array}{c}
2 j \\
i
\end{array}\right]_{q^{2}}\left[\begin{array}{c}
j+k-1 \\
k
\end{array}\right]_{q^{8}}\left[\begin{array}{c}
j+l-1 \\
l
\end{array}\right]_{q^{4}} \\
& \times\left[\begin{array}{c}
n-2 j-i-2 k-l \\
j
\end{array}\right]_{q^{4}} \\
& = \begin{cases}\sum_{k} q^{24 k^{2}+2 k}\left[\begin{array}{c}
2 n \\
n+3 k
\end{array}\right]_{q^{4}}-q^{24 k^{2}+22 k+5}\left[\begin{array}{c}
2 n-1 \\
n+3 k+1
\end{array}\right]_{q^{4}} & \text { if } n=2 m, \\
\sum_{k} q^{24 k^{2}+2 k}\left[\begin{array}{c}
2 n \\
n+3 k
\end{array}\right]_{q^{4}}-q^{24 k^{2}+22 k+5}\left[\begin{array}{c}
2 n+1 \\
n+3 k+2
\end{array}\right]_{q^{4}} & \text { if } n=2 m+1 .\end{cases} \\
& P_{0}=P_{1}=1 \\
& P_{2}=q^{4}+1 \\
& P_{3}=-q^{7}-q^{5}+q^{4}+1 \\
& P_{n}=\left(1-q^{4}\right) P_{n-1}+\left(q^{4 n-4}+2 q^{4}\right) P_{n-2}+\left(q^{8}-q^{4}-q^{4 n-5}-q^{4 n-7}\right) P_{n-3} \\
& +\left(q^{4 n-8}-q^{8}\right) P_{n-4} \text { if } n \geqq 4
\end{aligned}
$$

\section{Identity 3.54 (Finite form of A.54).}

$$
\begin{gathered}
1+\sum_{j \geqq 1} \sum_{i \geqq 0} \sum_{k \geqq 0} \sum_{l \geqq 0}(-1)^{l} q^{j^{2}+i^{2}+i+k+l}\left[\begin{array}{c}
j-1 \\
i
\end{array}\right]_{q^{2}}\left[\begin{array}{c}
j+k-1 \\
k
\end{array}\right]_{q^{2}}\left[\begin{array}{c}
j+l-2 \\
l
\end{array}\right]_{q} \\
\times\left[\begin{array}{c}
n-j-2 k-2 i-l] \\
j
\end{array}\right]_{q} \\
=\sum_{j=-\infty}^{\infty}(-1)^{j} q^{6 j^{2}+j}\left[\begin{array}{c}
n \\
\left\lfloor\frac{n+6 j+1}{2}\right\rfloor
\end{array}\right] \\
P_{0}=P_{1}=1 \\
P_{2}=q+1 \\
P_{3}=q^{2}+q+1 \\
P_{4}=q^{4}+q^{3}+2 q^{2}+q+1 \\
P_{n}=P_{n-1}+\left(q+q^{2}+q^{n-1}\right) P_{n-2}-\left(q+q^{2}+q^{n-1}\right) P_{n-3}+\left(q^{n-1}-q^{3}\right) P_{n-4} \\
\left(q^{3}-q^{n-1}\right) P_{n-5} \text { if } n \geqq 5
\end{gathered}
$$

Identity 3.55 (Finite form of A.55). Bosonic representation for even $n$ conjectured 
by Santos [60, p. 75, eqn. 6.33].

$$
\begin{aligned}
& \sum_{i \geqq 0} \sum_{I \geqq 0} \sum_{j \geqq 0} \sum_{k \geqq 0} \sum_{K \geqq 0}(-1)^{i+I+K} q^{4 j^{2}+4 j+2 i^{2}-i+2 I^{2}+I+4 k+4 K}\left[\begin{array}{c}
j+1 \\
i
\end{array}\right]_{q^{4}}\left[\begin{array}{c}
j \\
I
\end{array}\right]_{q^{4}}\left[\begin{array}{c}
j+k \\
k
\end{array}\right]_{q^{8}} \\
& \times\left[\begin{array}{c}
j+K-1 \\
K
\end{array}\right]_{q^{4}}\left[\begin{array}{c}
n-j-i-I-2 k-K \\
j
\end{array}\right]_{q^{4}} \\
& = \begin{cases}\sum_{k} q^{24 k^{2}+10 k}\left[\begin{array}{c}
2 n+1 \\
n+3 k+1
\end{array}\right]_{q^{4}}-q^{24 k^{2}+14 k+1}\left[\begin{array}{c}
2 n \\
n+3 k+1
\end{array}\right]_{q^{4}} & \text { if } n=2 m, \\
\sum_{k} q^{24 k^{2}+10 k}\left[\begin{array}{c}
2 n+1 \\
n+3 k+1
\end{array}\right]_{q^{4}}-q^{24 k^{2}+14 k+1}\left[\begin{array}{c}
2 n+2 \\
n+3 k+2
\end{array}\right]_{q^{4}} & \text { if } n=2 m+1 .\end{cases} \\
& P_{0}=1 \\
& P_{1}=-q+1 \\
& P_{2}=q^{8}+q^{4}-q+1 \\
& P_{3}=-q^{13}-q^{11}-q^{9}+q^{8}-q^{5}+q^{4}-q+1 \\
& P_{n}=\left(1-q^{4}\right) P_{n-1}+\left(q^{4 n}+2 q^{4}\right) P_{n-2}+\left(q^{8}-q^{4}-q^{4 n-1}-q^{4 n-3}\right) P_{n-3} \\
& +\left(q^{4 n-4}-q^{8}\right) P_{n-4} \text { if } n \geqq 4
\end{aligned}
$$

Identity 3.56 (Finite form of A.56). Bosonic representation conjectured by Santos [60, p. 75, eqn. 6.34].

$$
\begin{gathered}
\sum_{i, j, k, l \geqq 0}(-1)^{i} q^{j^{2}+2 j+i^{2}+i+k+l}\left[\begin{array}{l}
j \\
i
\end{array}\right]_{q^{2}}\left[\begin{array}{c}
j+k \\
k
\end{array}\right]_{q^{2}}\left[\begin{array}{c}
j+l \\
l
\end{array}\right]_{q}\left[\begin{array}{c}
n-k-2 i-2 k-l \\
j
\end{array}\right]_{q} \\
=\sum_{j=-\infty}^{\infty} q^{6 j^{2}+5 j}\left[\begin{array}{c}
n+2 \\
\left\lfloor\frac{n+6 j+2}{2}\right\rfloor
\end{array}\right]_{q} \\
P_{0}=1 \\
P_{1}=q+1 \\
P_{2}=q^{3}+q^{2}+2 q+1 \\
P_{3}=q^{5}+2 q^{4}+2 q^{3}+2 q^{2}+2 q+1 \\
P_{n}=(1+q) P_{n-1}+q^{n+1} P_{n-2}+\left(-q-q^{2}\right) P_{n-3}+\left(q^{2}-q^{n-1}\right) P_{n-4} \text { if } n \geqq 4
\end{gathered}
$$

Observation 3.57 (Finite form of A.57). This identity is (55) with $q$ replaced by $-q$. Identity 3.58 (Finite form of A.58). Bosonic representation for even $n$ conjectured 
by Santos [60, p. 76, eqn. 6.36].

$$
\begin{gathered}
1+\sum_{j \geqq 1} \sum_{k \geqq 0} \sum_{l \geqq 0} q^{j^{2}+i(i+1) / 2+k}\left[\begin{array}{c}
j-1 \\
i
\end{array}\right]_{q}\left[\begin{array}{c}
j+k-1 \\
k
\end{array}\right]_{q^{2}}\left[\begin{array}{c}
n-i-j-2 k \\
j
\end{array}\right]_{q} \\
=\sum_{j=-\infty}^{\infty} q^{6 j^{2}+j}\left[\begin{array}{c}
n \\
\left\lfloor\frac{n+6 j+1}{2}\right\rfloor
\end{array}\right]_{q} \\
P_{0}=P_{1}=1 \\
P_{2}=q+1 \\
P_{3}=q^{2}+q+1 \\
P_{n}=(1+q) P_{n-1}+q^{n+1} P_{n-2}+\left(-q-q^{2}\right) P_{n-3}+\left(q^{2}-q^{n-1}\right) P_{n-4} \text { if } n \geqq 4
\end{gathered}
$$

Identity 3.59 (Finite form of A.59). Identity 3.59 is a special case of an identity due to Berkovich and McCoy [27, $p$. 59, eqn. (3.14) with $p=4, p^{\prime}=7, r=s=2, a=6$, $b=3,4]$.

$$
\begin{gathered}
\sum_{j \geqq 0} \sum_{k \geqq 0} q^{j^{2}+2 j+k}\left[\begin{array}{c}
j+k \\
k
\end{array}\right]_{q^{2}}\left[\begin{array}{c}
n-j-2 k \\
j
\end{array}\right]_{q}=\sum_{j=-\infty}^{\infty}(-1)^{j} q^{7 j^{2}+5 j}\left[\begin{array}{c}
n+2 \\
\left\lfloor\frac{n+7 j+5}{2}\right\rfloor
\end{array}\right]_{q} \\
P_{0}=P_{1}=1 \\
P_{2}=q^{3}+q+1 \\
P_{n}=P_{n-1}+\left(q+q^{n+1}\right) P_{n-2}-q P_{n-3} \text { if } n \geqq 3
\end{gathered}
$$

Identity 3.60 (Finite form of A.60). Identity 3.60 is a special case of an identity due to Berkovich and McCoy [27, p. 59, eqn. (3.14) with $p=4, p^{\prime}=7, r=s=2, s=3$, $a=4 ; b=4,5]$.

$$
\begin{gathered}
\sum_{j \geqq 0} \sum_{k \geqq 0} q^{j^{2}+j+k}\left[\begin{array}{c}
j+k \\
k
\end{array}\right]_{q^{2}}\left[\begin{array}{c}
n-j-2 k \\
j
\end{array}\right]_{q}=\sum_{j=-\infty}^{\infty}(-1)^{j} q^{7 j^{2}+3 j}\left[\begin{array}{c}
n+1 \\
\left\lfloor\frac{n+7 j+3}{2}\right\rfloor
\end{array}\right]_{q} \\
P_{0}=P_{1}=1 \\
P_{2}=q^{2}+q+1 \\
P_{n}=P_{n-1}+\left(q+q^{n}\right) P_{n-2}-q P_{n-3} \text { if } n \geqq 3
\end{gathered}
$$

Identity 3.61 (Finite form of A.61). Identity 3.61 is a special case of an identity due to Berkovich and McCoy [27, p. 59, eqn. (3.14) with $p=4, p^{\prime}=7, r=2, s=3, a=3,4$; $b=3]$.

$$
\begin{gathered}
\sum_{j \geqq 0} \sum_{k \geqq 0} q^{j^{2}+k}\left[\begin{array}{c}
j+k-1 \\
k
\end{array}\right]_{q^{2}}\left[\begin{array}{c}
n-j-2 k \\
j
\end{array}\right]_{q}=\sum_{j=-\infty}^{\infty}(-1)^{j} q^{7 j^{2}+j}\left[\begin{array}{c}
n \\
\left\lfloor\frac{n+7 j+1}{2}\right\rfloor
\end{array}\right]_{q} \\
P_{0}=P_{1}=1 \\
P_{2}=q+1 \\
P_{n}=P_{n-1}+\left(q+q^{n-1}\right) P_{n-2}-q P_{n-3} \text { if } n \geqq 3
\end{gathered}
$$


Observation 3.62. Identity (62) is equivalent to (46). (Andrews [9, p. 20, eqns. (8.5) and (8.6)])

Observation 3.63. Identity (63) is the same as (44).

Observation 3.64. Identity (64) is the same as (11).

Observation 3.65. Identity (65) is the equivalent to $(37)+\sqrt{q} \times(35)$.

Observation 3.66. Identity (66) is equivalent to $(71)+q \times(68)$.

Observation 3.67. Identity (67) is equivalent to (71) $-q \times(68)$.

Identity 3.68 (Finite form of A.68). Bosonic form conjectured by Santos [60, p. 79, eqn. 6.42].

$$
\begin{gathered}
\sum_{i \geqq 0} \sum_{j \geqq 0} \sum_{k \geqq 0} \sum_{l \geqq 0}(-1)^{k} q^{j^{2}+2 j+2 i^{2}+2 k+l}\left[\begin{array}{l}
j \\
i
\end{array}\right]_{q^{4}}\left[\begin{array}{c}
j+k \\
k
\end{array}\right]_{q^{2}}\left[\begin{array}{c}
j+l \\
l
\end{array}\right]_{q^{2}}\left[\begin{array}{c}
n-2 i-k-l \\
j
\end{array}\right]_{q^{2}} \\
=\sum_{j=-\infty}^{\infty}(-1)^{j} q^{8 j^{2}+6 j} \mathrm{U}(n+1,4 j+1 ; q) \\
P_{0}=1 \\
P_{1}=q^{3}-q^{2}+q+1 \\
P_{2}=q^{8}-q^{7}+q^{6}+2 q^{4}+q+1 \\
P_{n}=\left(1+q-q^{2}+q^{2 n+1}\right) P_{n-1}+\left(q^{3}+q^{2}-q\right) P_{n-2}+\left(q^{2 n+1}-q^{3}\right) P_{n-3} \text { if } n \geqq 3
\end{gathered}
$$

Identity 3.69 (Finite form of A.69). Bosonic form conjectured by Santos [60, p. 79, eqn. 6.43].

$$
\begin{gathered}
\sum_{i \geqq 0} \sum_{j \geqq 0} \sum_{k \geqq 0} \sum_{l \geqq 0}(-1)^{i} q^{j^{2}+2 j+2 i^{2}+2 i+k+2 l}\left[\begin{array}{l}
j \\
i
\end{array}\right]_{q^{4}}\left[\begin{array}{c}
j+k \\
k
\end{array}\right]_{q^{2}}\left[\begin{array}{c}
j+l \\
l
\end{array}\right]_{q^{2}}\left[\begin{array}{c}
n-2 i-k-l \\
j
\end{array}\right]_{q^{2}} \\
=\sum_{j=-\infty}^{\infty} q^{8 j^{2}+6 j} \mathrm{U}(n+1,4 j+1 ; q) \\
P_{0}=1 \\
P_{1}=q^{3}+q^{2}+q+1 \\
P_{2}=q^{8}+q^{7}+q^{6}+2 q^{5}+2 q^{4}+2 q^{3}+2 q^{2}+q+1 \\
P_{n}=\left(1+q+q^{2}+q^{2 n+1}\right) P_{n-1}-\left(q+q^{2}+q^{3}\right) P_{n-2}+\left(q^{3}-q^{2 n+1}\right) P_{n-3} \text { if } n \geqq 3
\end{gathered}
$$


Identity 3.70 (Finite form of A.70).

$$
\begin{gathered}
\sum_{i \geqq 0} \sum_{j \geqq 0} \sum_{k \geqq 0} \sum_{l \geqq 0}(-1)^{l} q^{j^{2}+2 j+2 i^{2}+k+2 l}\left[\begin{array}{l}
j \\
i
\end{array}\right]_{q^{4}}\left[\begin{array}{c}
j+k \\
k
\end{array}\right]_{q^{2}}\left[\begin{array}{c}
j+l-1 \\
l
\end{array}\right]_{q^{2}}\left[\begin{array}{c}
n-2 i-k-l \\
j
\end{array}\right]_{q^{2}} \\
=\sum_{j=-\infty}^{\infty}(-1)^{j} q^{8 j^{2}+4 j}\left[\mathrm{~T}_{0}(n, 4 j+1 ; q)+\mathrm{T}_{0}(n+1,4 j+1 ; q)\right] \\
P_{0}=1 \\
P_{1}=q^{3}+q+1 \\
P_{2}=q^{8}+q^{6}+q^{4}+q^{3}+q^{2}+q+1 \\
P_{n}=\left(1+q-q^{2}+q^{2 n+1}\right) P_{n-1}+\left(q^{3}+q^{2}-q\right) P_{n-2}+\left(q^{2 n-1}-q^{3}\right) P_{n-3} \text { if } n \geqq 3
\end{gathered}
$$

Identity 3.71 (Finite form of A.71). Bosonic representation conjectured by Santos [60, p. 80, eqn. 6.44].

$$
\begin{gathered}
1+\sum_{j \geqq 1} \sum_{i \geqq 0} \sum_{k \geqq 0} \sum_{l \geqq 0}(-1)^{l} q^{j^{2}+2 i^{2}+2 i+k+2 l}\left[\begin{array}{c}
j-1 \\
i
\end{array}\right]_{q^{4}}\left[\begin{array}{c}
j+k-1 \\
k
\end{array}\right]_{q^{2}} \\
\times\left[\begin{array}{c}
j+l-2 \\
l
\end{array}\right]_{q^{2}}\left[\begin{array}{c}
n-2 i-k-l \\
j
\end{array}\right]_{q^{2}} \\
=\sum_{j=-\infty}^{\infty}(-1)^{j} q^{8 j^{2}+2 j} \mathrm{U}(n, 4 j ; q) \\
P_{0}=1 \\
P_{1}=q+1 \\
P_{2}=q^{4}+q^{3}+q^{2}+q+1 \\
P_{n}=\left(1+q+q^{2 n-1}\right) P_{n-1}+\left(q^{4}-q-q^{2 n-1}\right) P_{n-2}-\left(q^{4}+q^{5}-q^{2 n-1}\right) P_{n-3} \\
+\left(q^{5}-q^{2 n-1}\right) P_{n-4} \text { if } n \geqq 4
\end{gathered}
$$

Identity 3.72 (Finite form of A.72). Bosonic representation conjectured by Santos [60, p. 80, eqn. 6.45].

$$
\begin{gathered}
1+\sum_{j \geqq 1} \sum_{i \geqq 0} \sum_{k \geqq 0} \sum_{l \geqq 0}(-1)^{i} q^{j^{2}+2 i^{2}+2 i+k+2 l}\left[\begin{array}{c}
j-1 \\
i
\end{array}\right]_{q^{4}}\left[\begin{array}{c}
j+k-1 \\
k
\end{array}\right]_{q^{2}} \\
\times\left[\begin{array}{c}
j+l-2 \\
l
\end{array}\right]_{q^{2}}\left[\begin{array}{c}
n-2 i-k-l \\
j
\end{array}\right]_{q^{2}} \\
=\sum_{j=-\infty}^{\infty} q^{8 j^{2}+2 j} \mathrm{U}(n, 4 j ; q) \\
P_{0}=1 \\
P_{1}=q+1
\end{gathered}
$$




$$
\begin{gathered}
P_{2}=q^{4}+q^{3}+q^{2}+q+1 \\
P_{3}=q^{9}+q^{8}+q^{7}+2 q^{6}+2 q^{5}+2 q^{4}+2 q^{3}+q^{2}+q+1 \\
P_{n}=\left(1+q^{2}+q^{2 n-1}\right) P_{n-1}+q^{2 n-2} P_{n-2}-\left(q^{2 n-1}+q^{4}+q^{2}\right) P_{n-3} \\
+\left(q^{4}-q^{2 n-2}\right) P_{n-4} \text { if } n \geqq 4
\end{gathered}
$$

Observation 3.73. Identity (73) is equivalent to $(77)+(78)$ and to $(77)+(75)+q \times(76)$.

Observation 3.74. Identity (74) is equivalent to $(77)+(78)-q \times(76)$ and to $(77)+(75)$.

Identity 3.75 (Finite form of A.75). Note: (75) is (78) $-q \times(76)$. Bosonic representation conjectured by Santos [60, p. 81, eqn. (6.46)].

$$
\begin{gathered}
1+\sum_{j \geqq 1} \sum_{i \geqq 0} \sum_{I \geqq 0} \sum_{k \geqq 0} \sum_{K \geqq 0} q^{j(j+1) / 2+i(3 i+1) / 2+I(I+1) / 2+2 k+K}\left[\begin{array}{c}
j-1 \\
i
\end{array}\right]_{q^{3}}\left[\begin{array}{l}
j \\
I
\end{array}\right]_{q}\left[\begin{array}{c}
j+k-1 \\
k
\end{array}\right]_{q^{2}} \\
\times\left[\begin{array}{c}
j+K-1 \\
K
\end{array}\right]_{q^{2}}\left[\begin{array}{c}
n-1-3 i-I-2 k-2 K \\
j-1
\end{array}\right]_{q} \\
\sum_{j=-\infty}^{\infty}(-1)^{j} q^{9 j^{2}} \mathrm{~T}_{1}(n+1,6 j ; \sqrt{q})-(-1)^{j} q^{9 j^{2}+6 j+1} \mathrm{~T}_{1}(n+1,6 j+2 ; \sqrt{q}) \\
P_{0}=1 \\
P_{1}=q+1 \\
P_{2}=q^{3}+q^{2}+q+1 \\
P_{3}=q^{6}+q^{5}+2 q^{4}+2 q^{3}+2 q^{2}+q+1 \\
P_{n}=\left(1+q+q^{n}\right) P_{n-1}-\left(q+q^{2}\right) P_{n-3}+\left(q^{2}-q^{n}\right) P_{n-4} \text { if } n \geqq 4
\end{gathered}
$$

Identity 3.76 (Finite form of A.76).

$$
\begin{gathered}
\sum_{i \geqq 0} \sum_{I \geqq 0} \sum_{j \geqq 0} \sum_{k \geqq 0} \sum_{K \geqq 0}(-1)^{i} q^{j(j+3) / 2+3 i(i+1) / 2 I(I+1) / 2+k+2 K}\left[\begin{array}{l}
j \\
i
\end{array}\right]_{q^{3}}\left[\begin{array}{c}
j+1 \\
I
\end{array}\right]_{q}\left[\begin{array}{c}
j+k \\
k
\end{array}\right]_{q^{2}} \\
\times\left[\begin{array}{c}
j+K \\
K
\end{array}\right]_{q^{2}}\left[\begin{array}{c}
n-3 i-I-2 k-2 K \\
j
\end{array}\right]_{q} \\
=\sum_{j=-\infty}^{\infty}(-1)^{j} q^{9 j^{2}+6 j} \mathrm{~T}_{1}(n+2,6 j+2 ; \sqrt{q}) \\
P_{0}=1 \\
P_{1}=q^{2}+q+1 \\
P_{2}=q^{5}+q^{4}+2 q^{3}+2 q^{2}+2 q+1 \\
P_{4}=q^{14}+q^{13}+2 q^{12}+3 q^{11}+5 q^{10}+6 q^{9}+7 q^{8}+8 q^{7}+9 q^{6}+8 q^{5}+7 q^{4}+5 q^{3} \\
+4 q^{2}+2 q+1 \\
P_{n}=\left(1+q^{n+1}\right) P_{n-1}+\left(q+q^{2}+q^{n+1}\right) P_{n-2}-\left(q^{2}+q\right) P_{n-3} \\
-\left(q^{3}+q^{n+1}\right) P_{n-4}+\left(q^{3}-q^{n+1}\right) P_{n-5} \text { if } n \geqq 5
\end{gathered}
$$


Identity 3.77 (Finite form of A.77).

$$
\begin{gathered}
\sum_{i \geqq 0} \sum_{j \geqq 0} \sum_{k \geqq 0} \sum_{l \geqq 0}(-1)^{i} q^{\left(j^{2}+j+3 i^{2}+3 i+2 k+2 l\right) / 2}\left[\begin{array}{l}
j \\
i
\end{array}\right]_{q^{3}}\left[\begin{array}{c}
j+k \\
k
\end{array}\right]_{q^{2}}\left[\begin{array}{c}
j+l-1 \\
l
\end{array}\right]_{q}\left[\begin{array}{c}
n-3 i-2 k-l \\
j
\end{array}\right]_{q} \\
=\sum_{j=-\infty}^{\infty}(-1)^{j} q^{9 j^{2}+3 j} \mathrm{~T}_{1}(n+1,6 j+1 ; \sqrt{q}) \\
P_{0}=1 \\
P_{1}=q+1 \\
P_{2}=q^{3}+2 q^{2}+2 q+1 \\
P_{n}=\left(1+q^{n}\right) P_{n-1}+\left(q+q^{n}\right) P_{n-2}+\left(q^{n}-q\right) P_{n-3} \text { if } n \geqq 3
\end{gathered}
$$

Observation 3.78. Identity (78) is equivalent to $(75)+q \times(76)$.

Identity 3.79 (Finite form of A.79/98). Eqn. (3.79-b) due to Andrews [10, p. 80, eqn. 8.42].

$$
\begin{gathered}
\sum_{j \geqq 0} q^{j^{2}}\left[\begin{array}{c}
n+j \\
2 j
\end{array}\right]_{q}=\sum_{k=-\infty}^{\infty} q^{15 k^{2}+k}\left[\begin{array}{c}
2 n \\
n+5 k
\end{array}\right]_{q}-q^{15 k^{2}+11 k+2}\left[\begin{array}{c}
2 n \\
n+5 k+2
\end{array}\right]_{q} \\
=\sum_{j=-\infty}^{\infty}(-1)^{j} q^{10 j^{2}+2 j} \mathrm{U}(n, 5 j ; q) \\
P_{0}=1 \\
P_{1}=q+1 \\
P_{n}=\left(1+q+q^{2 n-1}\right) P_{n-1}-q P_{n-2} \text { if } n \geqq 2
\end{gathered}
$$

Identity 3.80 (Finite form of A.80). Bosonic representation conjectured by Santos [60, p. 77, eqn. 6.39].

$$
\begin{gathered}
\sum_{j \geqq 0} \sum_{k \geqq 0} q^{j(j+1) / 2+k}\left[\begin{array}{c}
j+k \\
k
\end{array}\right]_{q^{2}}\left[\begin{array}{c}
n-2 k \\
j
\end{array}\right]_{q} \\
=\sum_{k=-\infty}^{\infty} q^{\left(21 k^{2}+5 k\right) / 2} \mathrm{~T}_{1}(n+1,7 k+1 ; \sqrt{q})-q^{(21 k+19) k / 2+2} \mathrm{~T}_{1}(n+1,7 k+3 ; \sqrt{q}) \\
P_{0}=1 \\
P_{1}=q+1 \\
P_{2}=q^{3}+q^{2}+2 q+1 \\
P_{n}=\left(1+q^{n}\right) P_{n-1}+q P_{n-2}-q P_{n-3} \text { if } n \geqq 3
\end{gathered}
$$

Identity 3.81 (Finite form of A.81). Bosonic representation conjectured by Santos [60, 
p. 81, eqn. 6.47].

$$
\begin{gathered}
\sum_{j \geqq 0} \sum_{k \geqq 0} q^{j(j+1) / 2+k}\left[\begin{array}{c}
j+k-1 \\
k
\end{array}\right]_{q^{2}}\left[\begin{array}{c}
n-2 k \\
j
\end{array}\right]_{q} \\
=\sum_{k=-\infty}^{\infty} q^{\left(21 k^{2}+k\right) / 2} \mathrm{~T}_{1}(n+1,7 k ; \sqrt{q})-q^{\left(21 k^{2}+13 k+2\right) / 2} \mathrm{~T}_{1}(n+1,7 k+2 ; \sqrt{q}) \\
P_{0}=1 \\
P_{1}=q+1 \\
P_{2}=q^{3}+q^{2}+q+1 \\
P_{n}=\left(1+q^{n}\right) P_{n-1}+q P_{n-2}-q P_{n-3} \text { if } n \geqq 3
\end{gathered}
$$

Identity 3.82 (Finite form of A.82). Bosonic representation conjectured by Santos [60, p. 82, eqn. 6.48].

$$
\begin{gathered}
\sum_{j \geqq 0} \sum_{k \geqq 0} q^{j(j+3) / 2+k}\left[\begin{array}{c}
j+k \\
k
\end{array}\right]_{q^{2}}\left[\begin{array}{c}
n-2 k \\
j
\end{array}\right]_{q} \\
=\sum_{k=-\infty}^{\infty} q^{\left(21 k^{2}+11 k\right) / 2} \mathrm{~T}_{1}(n+2,7 k+2 ; \sqrt{q})-q^{\left(21 k^{2}+17 k+2\right) / 2} \mathrm{~T}_{1}(n+2,7 k+3 ; \sqrt{q}) \\
P_{0}=1 \\
P_{1}=q^{2}+1 \\
P_{2}=q^{5}+q^{3}+q^{2}+q+1 \\
P_{n}=\left(1+q^{n+1}\right) P_{n-1}+q P_{n-2}-q P_{n-3} \text { if } n \geqq 3
\end{gathered}
$$

Observation 3.83. Identity (83) is the same as (39).

Observation 3.84. Identity (84) is the same as (9).

Observation 3.85. Identity (85) is the same as (52).

Observation 3.86. Identity (86) is the same as (38).

Observation 3.87. Identity (87) is the same as (27).

Observation 3.88. Identity (88) is equivalent to $(91)-q^{2} \times(90)$.

Observation 3.89. Identity (89) is equivalent to (93) $-q \times(91)$.

Identity 3.90 (Finite form of A.90). Bosonic representation conjectured by Santos [60, 
p. 83, eqn. 6.52].

$$
\begin{gathered}
\sum_{i \geqq 0} \sum_{j \geqq 0} \sum_{k \geqq 0}(-1)^{i} q^{j^{2}+3 j+3 i(i+1) / 2+k}\left[\begin{array}{l}
j \\
i
\end{array}\right]_{q^{3}}\left[\begin{array}{c}
2 j+k+1 \\
k
\end{array}\right]_{q}\left[\begin{array}{c}
n-j-3 i-2 k \\
j
\end{array}\right]_{q} \\
=\sum_{j=-\infty}^{\infty}(-1)^{j} q^{\left(27 j^{2}+21 j\right) / 2}\left[\begin{array}{c}
n+3 \\
\left\lfloor\frac{n+9 j+7}{2}\right\rfloor
\end{array}\right]_{q} \\
P_{0}=1 \\
P_{1}=1 \\
P_{2}=q^{4}+q^{2}+q+1 \\
P_{3}=q^{5}+q^{4}+q^{2}+q+1 \\
P_{4}=q^{10}+q^{8}+q^{7}+2 q^{6}+2 q^{5}+2 q^{4}+q^{3}+2 q^{2}+q+1 \\
P_{n}=P_{n-1}+\left(q+q^{2}+q^{n+2}\right) P_{n-2}-\left(q^{2}+q\right) P_{n-3}-q^{3} P_{n-4} \\
+\left(q^{3}-q^{n+2}\right) P_{n-5} \text { if } n \geqq 5
\end{gathered}
$$

Identity 3.91 (Finite form of A.91). Bosonic representation conjectured for even $n$ by Santos [60, p. 84, eqn. 6.53].

$$
\begin{gathered}
\sum_{i \geqq 0} \sum_{j \geqq 0} \sum_{k \geqq 0}(-1)^{i} q^{j^{2}+2 j+3 i(i+1) / 2+k}\left[\begin{array}{l}
j \\
i
\end{array}\right]_{q^{3}}\left[\begin{array}{c}
2 j+k+1 \\
k
\end{array}\right]_{q}\left[\begin{array}{c}
n-j-3 i-2 k \\
j
\end{array}\right]_{q} \\
=\sum_{j=-\infty}^{\infty}(-1)^{j} q^{\left(27 j^{2}+15 j\right) / 2}\left[\begin{array}{c}
n+2 \\
\left\lfloor\frac{n+9 j+5}{2}\right\rfloor
\end{array}\right]_{q} \\
P_{0}=1 \\
P_{1}=1 \\
P_{2}=q^{3}+q^{2}+q+1 \\
P_{3}=q^{4}+q^{3}+q^{2}+q+1 \\
P_{4}=q^{8}+q^{7}+q^{6}+2 q^{5}+3 q^{4}+2 q^{3}+2 q^{2}+q+1 \\
P_{n}=P_{n-1}+\left(q+q^{2}+q^{n+1}\right) P_{n-2}-\left(q^{2}+q\right) P_{n-3}-q^{3} P_{n-4} \\
+\left(q^{3}-q^{n+1}\right) P_{n-5} \text { if } n \geqq 5
\end{gathered}
$$

Identity 3.92 (Finite form of A.92). Bosonic representation conjectured by Santos [60, p. 84, eqn 6.54].

$$
\begin{aligned}
& \sum_{i \geqq 0} \sum_{j \geqq 0} \sum_{k \geqq 0}(-1)^{i} q^{j^{2}+j+3 i(i+1) / 2+k}\left[\begin{array}{l}
j \\
i
\end{array}\right]_{q^{3}}\left[\begin{array}{c}
2 j+k \\
k
\end{array}\right]_{q}\left[\begin{array}{c}
n-j-3 i-2 k \\
j
\end{array}\right]_{q} \\
& =\sum_{j=-\infty}^{\infty}(-1)^{j} q^{\left(27 j^{2}+9 j\right) / 2}\left[\begin{array}{c}
n+1 \\
\left\lfloor\frac{n+9 j+3}{2}\right\rfloor
\end{array}\right]_{q} \\
& P_{0}=1
\end{aligned}
$$




$$
\begin{gathered}
P_{1}=1 \\
P_{2}=q^{2}+q+1 \\
P_{3}=q^{3}+q^{2}+q+1 \\
P_{n}=(1-q) P_{n-1}+\left(2 q+q^{n}\right) P_{n-2}+\left(q-q^{2}+q^{n}\right) P_{n-3}+\left(q^{n}-q^{2}\right) P_{n-4} \text { if } n \geqq 4
\end{gathered}
$$

Identity 3.93 (Finite form of A.93). Bosonic representation for even $n$ conjectured by Santos [60, p. 85, eqn 6.55].

$$
\begin{gathered}
\sum_{j \geqq 0} \sum_{i \geqq 0} \sum_{k \geqq 0}(-1)^{i} q^{j^{2}+3 i(i+1) / 2+k}\left[\begin{array}{c}
j-1 \\
i
\end{array}\right]_{q^{3}}\left[\begin{array}{c}
2 j+k-2 \\
k
\end{array}\right]_{q}\left[\begin{array}{c}
n-3 i-j-2 k \\
j
\end{array}\right]_{q} \\
=\sum_{j=-\infty}^{\infty}(-1)^{j} q^{\left(27 j^{2}+3 j\right) / 2}\left[\begin{array}{c}
n \\
\left\lfloor\frac{n+9 j+1}{2}\right\rfloor
\end{array}\right]_{q} \\
P_{0}=1 \\
P_{1}=1 \\
P_{2}=q+1 \\
P_{3}=q^{2}+q+1 \\
P_{4}=q^{4}+q^{3}+2 q^{2}+q+1 \\
P_{n}=P_{n-1}+\left(q+q^{2}+q^{n-1}\right) P_{n-2}-\left(q^{2}+q\right) P_{n-3}-q^{3} P_{n-4} \\
+\left(q^{3}-q^{n-1}\right) P_{n-5} \text { if } n \geqq 5
\end{gathered}
$$

Identity 3.94 (Finite form of A.94). Eqn. (3.94-b) was not stated explicilty but was indicated indirectly by Andrews [7, p. 5291] as the dual of eqn. (4.1) for odd $N$.

$$
\begin{gathered}
\sum_{j \geqq 0} q^{j(j+1)}\left[\begin{array}{c}
n+j+1 \\
2 j+1
\end{array}\right]_{q} \\
=\sum_{k=-\infty}^{\infty} q^{15 k^{2}+4 k}\left[\begin{array}{c}
2 n+1 \\
n+5 k+1
\end{array}\right]_{q}-q^{15 k^{2}+14 k+3}\left[\begin{array}{c}
2 n+1 \\
n+5 k+3
\end{array}\right]_{q} \\
=\sum_{j=-\infty}^{\infty}(-1)^{j} q^{10 j^{2}+3 j} \mathrm{~T}_{1}(n+1,5 j+1 ; q)+\sum_{j=-\infty}^{\infty}(-1)^{j} q^{10 j^{2}+7 j+1} \mathrm{~T}_{1}(n+1,5 j+2 ; q) \\
P_{0}=1 \\
P_{1}=q^{2}+q+1 \\
P_{n}=\left(1+q+q^{2 n}\right) P_{n-1}-q P_{n-2} \text { if } n \geqq 2
\end{gathered}
$$

Identity 3.95 (Finite form of A.95). Bosonic representation conjectured by Santos [60, 
p. 86, eqn. 6.57].

$$
\begin{gathered}
\sum_{j \geqq 0} \sum_{k \geqq 0} \sum_{l \geqq 0}(-1)^{l} q^{3 j^{2}-2 j+k+2 l}\left[\begin{array}{c}
j+k-1 \\
k
\end{array}\right]_{q^{2}}\left[\begin{array}{c}
j+l-1 \\
l
\end{array}\right]_{q^{2}}\left[\begin{array}{c}
n-2 j-k-l \\
j
\end{array}\right]_{q^{2}} \\
=\sum_{k=-\infty}^{\infty} q^{15 k^{2}+4 k} \mathrm{U}(n-2,5 k ; q)-q^{15 k^{2}+14 k+3} \mathrm{U}(n-2,5 k+2 ; q) \\
P_{0}=P_{1}=P_{2}=1 \\
P_{n}=\left(1+q-q^{2}\right) P_{n-1}+\left(q^{3}+q^{2}-q\right) P_{n-2}+\left(q^{2 n-5}-q^{3}\right) P_{n-3} \text { if } n \geqq 3
\end{gathered}
$$

Identity 3.96 (Finite form of A.96). Eqn. (3.96-b) was not stated explicilty but was indicated indirectly by Andrews [7, p. 5291] as the dual of eqn. (4.2) for odd $N$.

$$
\begin{gathered}
\sum_{j \geqq 0} q^{j(j+2)}\left[\begin{array}{c}
n+j+1 \\
2 j+1
\end{array}\right]_{q} \\
=\sum_{k=-\infty}^{\infty} q^{15 k^{2}+7 k}\left[\begin{array}{c}
2 n+2 \\
n+5 k+2
\end{array}\right]_{q}-q^{15 k^{2}+13 k+2}\left[\begin{array}{c}
2 n+2 \\
n+5 k+3
\end{array}\right]_{q} \\
=\sum_{j=-\infty}^{\infty}(-1)^{j} q^{10 j^{2}+6 j} \mathrm{U}(n+1,5 j+1 ; q) \\
P_{0}=1 \\
P_{1}=q^{3}+q+1 \\
P_{n}=\left(1+q+q^{2 n+1}\right) P_{n-1}-q P_{n-2} \text { if } n \geqq 2
\end{gathered}
$$

Observation 3.97. Identity (97) is equivalent to (95).

Observation 3.98. Identity (98) is the same as (79).

Identity 3.99 (Finite forms of A.99). Eqn. (3.99-b) was not stated explicilty but was indicated indirectly by Andrews [7, p. 5291] as the dual of eqn. (4.2) for even $N$.

$$
\begin{gathered}
\sum_{j \geqq 0} q^{j(j+1)}\left[\begin{array}{c}
n+j \\
2 j
\end{array}\right]_{q} \\
=\sum_{k=-\infty}^{\infty} q^{15 k^{2}+2 k}\left[\begin{array}{c}
2 n+1 \\
n+5 k+1
\end{array}\right]_{q}-q^{15 k^{2}+8 k+1}\left[\begin{array}{c}
2 n+1 \\
n+5 k+2
\end{array}\right]_{q} \\
=\sum_{j=-\infty}^{\infty}(-1)^{j} q^{10 j^{2}+j} \mathrm{~T}_{1}(n+1,5 j ; q)-(-1)^{j} q^{10 j^{2}+9 j+2} \mathrm{~T}_{1}(n+1,5 j+2 ; q) \\
P_{0}=1 \\
P_{1}=q^{2}+1 \\
P_{n}=\left(1+q+q^{2 n}\right) P_{n-1}-q P_{n-2} \text { if } n \geqq 2
\end{gathered}
$$




\section{Identity 3.100 (Finite form of A.100).}

$$
\begin{gathered}
\sum_{j \geqq 0} \sum_{k \geqq 0} \sum_{l \geqq 0}(-1)^{l} q^{3 j^{2}+k+2 l}\left[\begin{array}{c}
j+k-1 \\
k
\end{array}\right]_{q^{2}}\left[\begin{array}{c}
j+l-1 \\
l
\end{array}\right]_{q^{2}}\left[\begin{array}{c}
n-2 j-k-l \\
j
\end{array}\right]_{q^{2}} \\
=\sum_{k=-\infty}^{\infty} q^{15 k^{2}+2 k} \mathrm{U}(n-1,5 k ; q)-q^{15 k^{2}+8 k+1} \mathrm{U}(n-1,5 k+1 ; q) \\
P_{0}=P_{1}=P_{2}=1 \\
P_{n}=\left(1+q-q^{2}\right) P_{n-1}+\left(q^{3}+q^{2}-q\right) P_{n-2}+\left(q^{2 n-3}-q^{3}\right) P_{n-3} \text { if } n \geqq 3
\end{gathered}
$$

Observation 3.101. Identity (101) is the sum of (105-a) and (104).

Observation 3.102 (Finite form of A.102). Identity (102) is (105-a) $+q \times(103)$.

Identity 3.103 (Finite form of A.103).

$$
\begin{gathered}
\sum_{i \geqq 0} \sum_{I \geqq 0} \sum_{j \geqq 0} \sum_{k \geqq 0} \sum_{K \geqq 0}(-1)^{I} q^{j(j+3) / 2+i^{2}+i+I(I+1) / 2+k+K} \\
\times\left[\begin{array}{l}
j \\
i
\end{array}\right]_{q^{2}}\left[\begin{array}{l}
j \\
I
\end{array}\right]_{q}\left[\begin{array}{c}
j+k \\
k
\end{array}\right]_{q^{2}}\left[\begin{array}{c}
j+K \\
K
\end{array}\right]_{q}\left[\begin{array}{c}
n-2 i-I-2 k-K \\
j
\end{array}\right]_{q} \\
=\sum_{k=-\infty}^{\infty} q^{16 k^{2}+8 k} \mathrm{~T}_{1}(n+2,8 k+2 ; \sqrt{q})-q^{16 k^{2}+16 k+3} \mathrm{~T}_{1}(n+2,8 k+4 ; \sqrt{q}) \\
P_{0}=1 \\
P_{1}=q^{2}+q+1 \\
P_{2}=q^{5}+q^{4}+q^{3}+2 q^{2}+q+1 \\
P_{3}=\left(1+q+q^{9}+q^{8}+q^{7}+q^{6}+3 q^{5}+3 q^{4}+3 q^{3}+3 q^{2}+2 q+1\right.
\end{gathered}
$$

Identity 3.104 (Finite form of A.104).

$$
\begin{gathered}
1+\sum_{j \geqq 1} \sum_{i \geqq 0} \sum_{k \geqq 0} q^{j(j+1) / 2+i^{2}+i+k}\left[\begin{array}{c}
j-1 \\
i
\end{array}\right]_{q^{2}}\left[\begin{array}{c}
j+k-1 \\
k
\end{array}\right]_{q^{2}}\left[\begin{array}{c}
n-2 i-2 k \\
k
\end{array}\right]_{q} \\
=\sum_{k=-\infty}^{\infty} q^{16 k^{2}} \mathrm{~V}(n+1,8 k+1 ; \sqrt{q})-q^{16 k^{2}+8 k+1} \mathrm{~V}(n+1,8 k+3 ; \sqrt{q}) \\
P_{0}=1 \\
P_{1}=q+1 \\
P_{2}=q^{3}+q^{2}+q+1 \\
P_{n}=q^{6}+q^{5}+q^{4}+2 q^{3}+2 q^{2}+q+1 \\
P_{n}=\left(1+q+q^{n}\right) P_{n-1}-q^{n} P_{n-2}+\left(q^{n}-q^{2}-q\right) P_{n-3}+\left(q^{2}-q^{n}\right) P_{n-4} \text { if } n \geqq 4
\end{gathered}
$$


Observation 3.105. Identity (105) is the same as (37).

Identity 3.105-a (Finite form of Identity A.105-a).

$$
\begin{gathered}
\sum_{i \geqq 0} \sum_{j \geqq 0} \sum_{k \geqq 0} q^{j(j+1) / 2+i^{2}+i+k}\left[\begin{array}{l}
j \\
i
\end{array}\right]_{q^{2}}\left[\begin{array}{c}
j+k \\
k
\end{array}\right]_{q^{2}}\left[\begin{array}{c}
n-2 i-2 k \\
j
\end{array}\right]_{q} \\
=\sum_{j=-\infty}^{\infty}(-1)^{j} q^{4 j^{2}+2 j} \mathrm{~T}_{1}(n+1,4 j+1 ; \sqrt{q}) \\
P_{0}=1 \\
P_{1}=q+1 \\
P_{2}=q^{3}+q^{2}+2 q+1 \\
P_{n}=\left(1+q^{n}\right) P_{n-1}+q P_{n-2}+\left(q^{n}-q\right) P_{n-3} \text { if } n \geqq 3
\end{gathered}
$$

Observation 3.106. Identity (106) is the same as (35).

Identity 3.107 (Finite form of A.107).

$$
\begin{gathered}
\sum_{i \geqq 0} \sum_{j \geqq 0} \sum_{k \geqq 0} \sum_{l \geqq 0}(-1)^{i} q^{j^{2}+j+3 i^{2}+2 k+l}\left[\begin{array}{l}
j \\
i
\end{array}\right]_{q^{6}}\left[\begin{array}{c}
j+k \\
k
\end{array}\right]_{q^{4}}\left[\begin{array}{c}
j+l-1 \\
l
\end{array}\right]_{q^{2}}\left[\begin{array}{c}
n-3 i-2 k-l \\
j
\end{array}\right]_{q^{2}} \\
=\sum_{j=-\infty}^{\infty}(-1)^{j} q^{18 j^{2}+3 j} \mathrm{~V}(n+1,6 j+1 ; q)+(-1)^{j} q^{18 j^{2}+15 j+3} \mathrm{~V}(n+1,6 j+3 ; q) \\
P_{0}=1 \\
P_{1}=q^{2}+1 \\
P_{2}=q^{6}+q^{4}+q^{3}+2 q^{2}+1 \\
P_{n}=\left(1+q^{2 n}\right) P_{n-1}+\left(q^{2}+q^{2 n-1}\right) P_{n-2}+\left(q^{2 n-2}-q^{2}\right) P_{n-3} \text { if } n \geqq 3
\end{gathered}
$$

Observation 3.108. Identity (108) is equivalent to $(115)-q^{2} \times(116)$.

Observation 3.109. Identity (109) is equivalent to $(109-\mathrm{a})+q \times(110)$.

Identity 3.109-a (Finite form of Identity A.109-a).

$$
\begin{gathered}
\sum_{i \geqq 0} \sum_{j \geqq 0} \sum_{k \geqq 0} \sum_{K \geqq 0} \sum_{l \geqq 0}(-1)^{i+L} q^{j^{2}+3 i^{2}+k+K+2 L}\left[\begin{array}{l}
j \\
i
\end{array}\right]_{q^{6}}\left[\begin{array}{c}
j+k-1 \\
k
\end{array}\right]_{q^{2}}\left[\begin{array}{c}
j+K-1 \\
K
\end{array}\right]_{q^{2}} \\
\times\left[\begin{array}{c}
j+L-1 \\
L
\end{array}\right]_{q^{2}}\left[\begin{array}{c}
n-3 i-k-K-L \\
j
\end{array}\right]_{q^{2}} \\
=\sum_{j=-\infty}^{\infty}(-1)^{j} q^{18 j^{2}}\left[\mathrm{~T}_{0}(n, 6 j ; q)+\mathrm{T}_{0}(n-1,6 j ; q)\right] \\
+(-1)^{j} q^{18 j^{2}+12 j+2}\left[\mathrm{~T}_{0}(n, 6 j+2 ; q)+\mathrm{T}_{0}(n-1,6 j+2 ; q)\right]
\end{gathered}
$$




$$
\begin{gathered}
P_{0}=1 \\
P_{1}=q+1 \\
P_{2}=q^{4}+2 q^{2}+q+1 \\
P_{n}=\left(1+q-q^{2}+q^{2 n-1}\right) P_{n-1}+\left(q^{3}+q^{2}-q+q^{2 n-2}\right) P_{n-2}+\left(q^{2 n-3}-q^{3}\right) P_{n-3} \quad \text { if } n \geqq 3
\end{gathered}
$$

Identity 3.110 (Finite form of A.110).

$$
\begin{gathered}
\sum_{i \geqq 0} \sum_{j \geqq 0} \sum_{k \geqq 0} \sum_{K \geqq 0} \sum_{L \geqq 0}(-1)^{i+L} q^{j^{2}+2 j+3 i^{2}+k+K+2 L}\left[\begin{array}{c}
j \\
i
\end{array}\right]_{q^{6}}\left[\begin{array}{c}
j+k \\
k
\end{array}\right]_{q^{2}}\left[\begin{array}{c}
j+K-1 \\
K
\end{array}\right]_{q^{2}} \\
\times\left[\begin{array}{c}
j+L-1 \\
L
\end{array}\right]_{q^{2}}\left[\begin{array}{c}
n-3 i-k-K-L \\
j
\end{array}\right]_{q^{2}} \\
=\sum_{j=-\infty}^{\infty}(-1)^{j} q^{18 j^{2}+6 j}\left[\mathrm{~T}_{0}(n+1,6 j+1 ; q)+\mathrm{T}_{0}(n, 6 j+1 ; q)\right] \\
P_{0}=1 \\
P_{1}=q^{3}+q+1 \\
P_{n}=\left(1+q-q^{8}+q^{6}+2 q^{4}+q^{3}+q^{2}+q+1\right. \\
\text { if } n \geqq 3
\end{gathered}
$$

Observation 3.111. Identity (111) is equivalent to (114) $-q \times(115)$.

Observation 3.112. Identity (112) is equivalent to $(115)+q^{3} \times(116)$.

Observation 3.113. Identity (113) is equivalent to $(114)-q^{3} \times(115)$.

Identity 3.114 (Finite form of A.114). Bosonic representation conjectured by Santos [60, p. 89, eqn. 6.63].

$$
\begin{gathered}
1+\sum_{j \geqq 1} \sum_{i \geqq 0} \sum_{k \geqq 0} \sum_{K \geqq 0} \sum_{l \geqq 0}(-1)^{i+K} q^{j^{2}+3 i^{2}+3 i+k+2 K+2 l}\left[\begin{array}{c}
j-1 \\
i
\end{array}\right]_{q^{6}}\left[\begin{array}{c}
j+k-1 \\
k
\end{array}\right]_{q^{2}} \\
\times\left[\begin{array}{c}
j+K-2 \\
K
\end{array}\right]_{q^{2}}\left[\begin{array}{c}
j+l-1 \\
l
\end{array}\right]_{q^{2}}\left[\begin{array}{c}
n-1-3 i-k-K-k \\
j-1
\end{array}\right]_{q^{2}} \\
=\sum_{j=-\infty}^{\infty}(-1)^{j} q^{18 j^{2}+3 j} \mathrm{U}(n, 6 j ; q) \\
P_{0}=1 \\
P_{1}=q+1 \\
P_{3}=q^{4}+q^{3}+q^{2}+q+1 \\
P_{n}=\left(1+q+q^{9}+q^{8}+q^{7}+q^{6}+2 q^{5}+2 q^{4}+2 q^{3}+q^{2}+q+1\right. \\
+\left(q^{5}-q^{2 n-1}\right) P_{n-4} \text { if } n \geqq 3
\end{gathered}
$$


Identity 3.115 (Finite form of A.115). Bosonic representation conjectured by Santos [60, p. 89, eqn. 6.64].

$$
\begin{gathered}
\sum_{i \geqq 0} \sum_{j \geqq 0} \sum_{k \geqq 0} \sum_{K \geqq 0}(-1)^{i} q^{j^{2}+2 j+3 i^{2}+3 i+k+4 K}\left[\begin{array}{l}
j \\
i
\end{array}\right]_{q^{6}}\left[\begin{array}{c}
j+k \\
k
\end{array}\right]_{q^{4}}\left[\begin{array}{c}
j+K \\
K
\end{array}\right]_{q^{4}} \\
\times\left[\begin{array}{c}
n-3 i-k-2 K \\
j
\end{array}\right]_{q^{2}} \\
=\sum_{j=-\infty}^{\infty}(-1)^{j} q^{18 j^{2}+9 j} \mathrm{U}(n+1,6 j+1 ; q) \\
P_{0}=1 \\
P_{1}=q^{3}+q+1 \\
P_{2}=q^{8}+q^{6}+q^{5}+2 q^{4}+q^{3}+q^{2}+q+1 \\
P_{3}=q^{15}+q^{13}+q^{12}+2 q^{11}+q^{10}+2 q^{9}+2 q^{8}+3 q^{7} \\
+2 q^{6}+3 q^{5}+2 q^{4}+2 q^{3}+q^{2}+q+1 \\
P_{n}=\left(1+q+q^{2 n+1}\right) P_{n-1}+\left(q^{4}-q+\right) P_{n-2}-\left(q^{4}+q^{5}\right) P_{n-3}+\left(q^{5}-q^{2 n+1}\right) P_{n-4} \quad \text { if } n \geqq 3
\end{gathered}
$$

Identity 3.116 (Finite form of A.116). Bosonic representation conjectured by Santos [60, p. 90, eqn 6.65].

$$
\begin{gathered}
\sum_{i \geqq 0} \sum_{j \geqq 0} \sum_{k \geqq 0} \sum_{K \geqq 0}(-1)^{i} q^{j^{2}+4 j+3 i^{2}+3 i+k+4 K}\left[\begin{array}{l}
j \\
i
\end{array}\right]_{q^{6}}\left[\begin{array}{c}
j+k \\
k
\end{array}\right]_{q^{2}}\left[\begin{array}{c}
j+K \\
K
\end{array}\right]_{q^{4}}\left[\begin{array}{c}
n-3 i-k-2 K \\
j
\end{array}\right]_{q^{2}} \\
=\sum_{j=-\infty}^{\infty}(-1)^{j} q^{18 j^{2}+15 j} \mathrm{U}(n+2,6 j+2 ; q) \\
P_{0}=1 \\
P_{1}=q^{5}+q+1 \\
P_{2}=q^{12}+q^{8}+q^{7}+q^{6}+q^{5}+q^{4}+q^{2}+q+1 \\
P_{3}=q^{21}+q^{17}+q^{16}+q^{15}+q^{14}+2 q^{13}+q^{12}+q^{11}+q^{10}+3 q^{9} \\
+2 q^{8}+2 q^{7}+q^{6}+2 q^{5}+q^{4}+q^{3}+q^{2}+q+1 \\
P_{n}=\left(1+q+q^{2 n+3}\right) P_{n-1}+\left(q^{4}-q\right) P_{n-2}-\left(q^{5}+q^{4}\right) P_{n-3}+\left(q^{5}-q^{2 n+3}\right) P_{n-4} \quad \text { if } n \geqq 4
\end{gathered}
$$

Identity 3.117 (Finite form of A.117). Bosonic representation conjectured by Santos [60, p. 88, eqn. 6.62].

$$
\begin{gathered}
\sum_{j \geqq 0} \sum_{k \geqq 0} \sum_{K \geqq 0}(-1)^{K} q^{j^{2}+k+2 K}\left[\begin{array}{c}
j+k-1 \\
k
\end{array}\right]_{q^{2}}\left[\begin{array}{c}
j+K-1 \\
K
\end{array}\right]_{q^{2}}\left[\begin{array}{c}
n-k-K \\
j
\end{array}\right]_{q^{2}} \\
=\sum_{k=-\infty}^{\infty} q^{21 k^{2}+2 k} \mathrm{U}(n, 7 k ; q)-q^{21 k^{2}+16 k+3} \mathrm{U}(n, 7 k+2 ; q) \\
P_{0}=1
\end{gathered}
$$




$$
\begin{gathered}
P_{1}=q+1 \\
P_{2}=q^{4}+q^{2}+q+1 \\
P_{n}=\left(1+q-q^{2}+q^{2 n-1}\right) P_{n-1}+\left(q^{3}+q^{2}-q\right) P_{n-2}-q^{3} P_{n-3} \quad \text { if } n \geqq 3
\end{gathered}
$$

Identity 3.118 (Finite form of A.118). Bosonic representation conjectured by Santos [60, p. 90, eqn. 6.65]

$$
\begin{gathered}
\sum_{j \geqq 0} \sum_{k \geqq 0} \sum_{K \geqq 0}(-1)^{K} q^{j^{2}+2 j+k+2 K}\left[\begin{array}{c}
j+k-1 \\
k
\end{array}\right]_{q^{2}}\left[\begin{array}{c}
j+K-1 \\
K
\end{array}\right]_{q^{2}}\left[\begin{array}{c}
n-k-K \\
j
\end{array}\right]_{q^{2}} \\
=\sum_{k=-\infty}^{\infty} q^{21 k^{2}+4 k} \mathrm{U}(n+1,7 k ; q)-q^{21 k^{2}+10 k+1} \mathrm{U}(n+1,7 k+1 ; q) \\
P_{0}=1 \\
P_{1}=q^{3}+1 \\
P_{2}=q^{8}+q^{4}+q^{3}+1 \\
P_{n}=\left(1+q-q^{2}+q^{2 n+1}\right) P_{n-1}+\left(q^{3}+q^{2}-q\right) P_{n-2}-q^{3} P_{n-3} \quad \text { if } n \geqq 3
\end{gathered}
$$

Identity 3.119 (Finite form of A.119). Bosonic representation conjectured by Santos [60, p. 91, eqn. 6.67].

$$
\begin{gathered}
\sum_{j \geqq 0} \sum_{k \geqq 0} \sum_{K \geqq 0}(-1)^{K} q^{j^{2}+2 j+k+2 K}\left[\begin{array}{c}
j+k \\
k
\end{array}\right]_{q^{2}}\left[\begin{array}{c}
j+K-1 \\
K
\end{array}\right]_{q^{2}}\left[\begin{array}{c}
n-k-K \\
j
\end{array}\right]_{q^{2}} \\
=\sum_{k=-\infty}^{\infty} q^{21 k^{2}+8 k} \mathrm{U}(n+1,7 k+1 ; q)-q^{21 k^{2}+20 k+4} \mathrm{U}(n+1,7 k+3 ; q) \\
P_{0}=1 \\
P_{1}=q^{3}+q+1 \\
P_{n}=\left(1+q-q^{2}+q^{2 n+1}\right) P_{n-1}+\left(q^{3}+q^{2}-q\right) P_{n-2}-q^{3} P_{n-3} \quad \text { if } n \geqq 3
\end{gathered}
$$

Identity 3.120 (Finite form of A.120). Bosonic representation conjectured by Santos [60, p. 91, eqn. 6.68].

$$
\begin{gathered}
1+\sum_{j \geqq 1} \sum_{i \geqq 0} \sum_{k \geqq 0} q^{j^{2}+j+i^{2}+i+k}\left[\begin{array}{c}
j-1 \\
i
\end{array}\right]_{q^{2}}\left[\begin{array}{c}
j+k-1 \\
k
\end{array}\right]_{q^{2}}\left[\begin{array}{c}
n-i-k \\
j
\end{array}\right]_{q^{2}} \\
=\sum_{k=-\infty}^{\infty} q^{24 k^{2}+2 k}\left[\begin{array}{c}
2 n+1 \\
n+6 k+1
\end{array}\right]_{q}-q^{24 k^{2}+10 k+1}\left[\begin{array}{c}
2 n+1 \\
n+6 k+2
\end{array}\right]_{q} \\
P_{0}=1 \\
P_{1}=q^{2}+1 \\
P_{2}=q^{6}+q^{4}+q^{3}+q^{2}+1 \\
P_{n}=\left(1+q+q^{2}+q^{2 n}\right) P_{n-1}-\left(q^{3}+q^{2}+q\right) P_{n-2}+\left(q^{3}-q^{2 n}\right) P_{n-3} \quad \text { if } n \geqq 3
\end{gathered}
$$


Identity 3.121 (Finite form of A.121). Bosonic representation conjectured by Santos [60, p. 92, eqn. 6.69].

$$
\begin{gathered}
1+\sum_{j \geqq 1} \sum_{i \geqq 0} \sum_{k \geqq 0} q^{j^{2}+i^{2}+i+k}\left[\begin{array}{c}
j-1 \\
i
\end{array}\right]_{q^{2}}\left[\begin{array}{c}
j+k-1 \\
k
\end{array}\right]_{q^{2}}\left[\begin{array}{c}
n-i-k \\
j
\end{array}\right]_{q^{2}} \\
=\sum_{k=-\infty}^{\infty} q^{24 k^{2}+2 k}\left[\begin{array}{c}
2 n+1 \\
n+6 k+1
\end{array}\right]_{q}-q^{24 k^{2}+14 k+2}\left[\begin{array}{c}
2 n+1 \\
n+6 k+2
\end{array}\right]_{q} \\
P_{0}=1 \\
P_{1}=q+1 \\
P_{2}=q^{4}+q^{3}+q^{2}+q+1 \\
P_{n}=\left(1+q+q^{2}+q^{2 n-1}\right) P_{n-1}-\left(q^{3}+q^{2}+q\right) P_{n-2}+\left(q^{3}-q^{2 n-1}\right) P_{n-3} \quad \text { if } n \geqq 3
\end{gathered}
$$

Identity 3.122 (Finite form of A.122). Bosonic representation conjectured by Santos [60, p. 92, eqn. 6.70]; corrected below.

$$
\begin{gathered}
\sum_{i, j, k \geqq 0} q^{j^{2}+3 j+i^{2}+i+k}\left[\begin{array}{l}
j \\
i
\end{array}\right]_{q^{2}}\left[\begin{array}{c}
j+k \\
k
\end{array}\right]_{q^{2}}\left[\begin{array}{c}
n+1-i-k \\
j+1
\end{array}\right]_{q^{2}} \\
=\sum_{k=-\infty}^{\infty} q^{24 k^{2}+14 k}\left[\begin{array}{c}
2 n+3 \\
n+6 k+3
\end{array}\right]_{q}-q^{24 k^{2}+22 k+3}\left[\begin{array}{c}
2 n+3 \\
n+6 k+4
\end{array}\right]_{q} \\
P_{0}=1 \\
P_{1}=q^{4}+q^{2}+q+1 \\
P_{n}=\left(1+q+q^{2}+q^{2 n+2}\right) P_{n-1}-\left(q^{3}+q^{2}+q\right) P_{n-2}+\left(q^{3}-q^{2 n+2}\right) P_{n-3} \quad \text { if } n \geqq 3
\end{gathered}
$$

Identity 3.123 (Finite form of A.123). Bosonic representation conjectured by Santos [60, p. 93, eqn. 6.71]; corrected below.

$$
\begin{gathered}
\sum_{i \geqq 0} \sum_{j \geqq 0} \sum_{k \geqq 0} \sum_{l \geqq 0}(-1)^{i} q^{2 j^{2}+2 j+2 i^{2}+2 i+k+2 l}\left[\begin{array}{l}
j \\
i
\end{array}\right]_{q^{4}}\left[\begin{array}{c}
j+k \\
k
\end{array}\right]_{q^{2}}\left[\begin{array}{c}
j+l \\
l
\end{array}\right]_{q^{2}}\left[\begin{array}{c}
n-2 i-k-l \\
j
\end{array}\right]_{q^{2}} \\
=\sum_{k=-\infty}^{\infty} q^{24 k^{2}+10 k}\left[\begin{array}{c}
2 n+2 \\
n+6 k+2
\end{array}\right]_{q}-q^{24 k^{2}+22 k+4}\left[\begin{array}{c}
2 n+2 \\
n+6 k+4
\end{array}\right]_{q} \\
P_{0}=1 \\
P_{1}=q^{3}+q^{2}+q+1 \\
P_{2}=q^{8}+q^{7}+q^{6}+2 q^{5}+2 q^{4}+2 q^{3}+2 q^{2}+q+1 \\
P_{n}=\left(1+q+q^{2}+q^{2 n+1}\right) P_{n-1}-\left(q^{3}+q^{2}+q\right) P_{n-2}+\left(q^{3}-q^{2 n+1}\right) P_{n-3} \quad \text { if } n \geqq 3
\end{gathered}
$$

Identity 3.124 (Finite form of A.124). Bosonic representation for even $n$ conjectured 
by Santos [60, p. 93, eqn. 6.72].

$$
\begin{aligned}
& \sum_{i \geqq 0} \sum_{j \geqq 0} \sum_{k \geqq 0} \sum_{K \geqq 0} \sum_{l \geqq 0} \sum_{L \geqq 0}(-1)^{i+K+l} q^{2 j^{2}+2 j+3 i^{2}+k+K+2 l+L}\left[\begin{array}{l}
j \\
i
\end{array}\right]_{q^{6}}\left[\begin{array}{c}
j+k \\
k
\end{array}\right]_{q^{2}}\left[\begin{array}{c}
j+K \\
K
\end{array}\right]_{q^{2}} \\
& \times\left[\begin{array}{c}
j+l-1 \\
l
\end{array}\right]_{q^{2}}\left[\begin{array}{c}
j+L-1 \\
L
\end{array}\right]_{q^{2}}\left[\begin{array}{c}
n-j-3 i-k-K-l-L \\
j
\end{array}\right]_{q^{2}}
\end{aligned}
$$

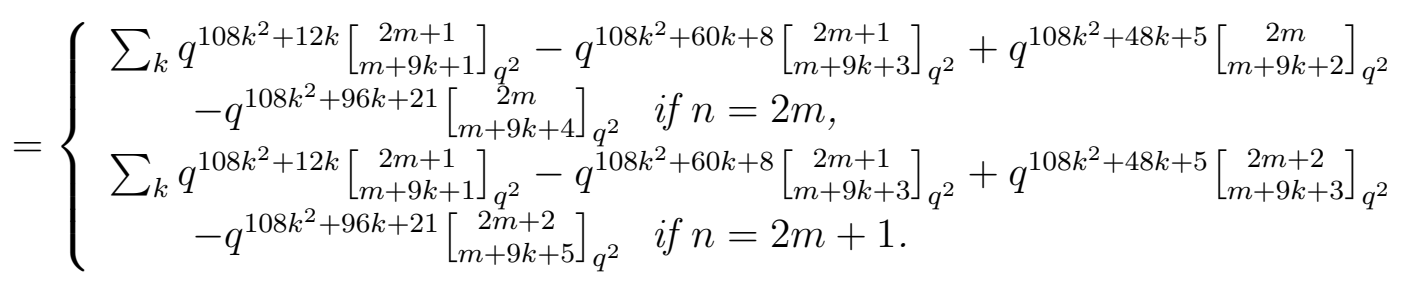

$$
\begin{aligned}
& P_{0}=1 \\
& P_{1}=1 \\
& P_{2}=q^{4}+q^{2}+1 \\
& P_{3}=q^{5}+q^{4}+q^{2}+1 \\
& P_{n}=\left(1-q^{2}\right) P_{n-1}+\left(2 q^{2}+q^{2 n}\right) P_{n-2}+\left(q^{4}-q^{2}+q^{2 n-1}\right) P_{n-3}+\left(q^{2 n-2}-q^{4}\right) P_{n-4} \quad \text { if } n \geqq 3
\end{aligned}
$$

Identity 3.125 (Finite form of A.125). Bosonic representation for even $n$ conjectured by Santos [60, p. 94, eqn. 6.73].

$$
\begin{aligned}
& \sum_{i \geqq 0} \sum_{j \geqq 0} \sum_{k \geqq 0} \sum_{K \geqq 0} \sum_{l \geqq 0} \sum_{L \geqq 0}(-1)^{i+K+l} q^{2 j^{2}+4 j+3 i^{2}+k+K+2 l+L}\left[\begin{array}{l}
j \\
i
\end{array}\right]_{q^{6}}\left[\begin{array}{c}
j+k \\
k
\end{array}\right]_{q^{2}}\left[\begin{array}{c}
j+K \\
K
\end{array}\right]_{q^{2}} \\
& \times\left[\begin{array}{c}
j+l-1 \\
l
\end{array}\right]_{q^{2}}\left[\begin{array}{c}
j+L-1 \\
L
\end{array}\right]_{q^{2}}\left[\begin{array}{c}
n-j-3 i-k-K-l-L \\
j
\end{array}\right]_{q^{2}}
\end{aligned}
$$

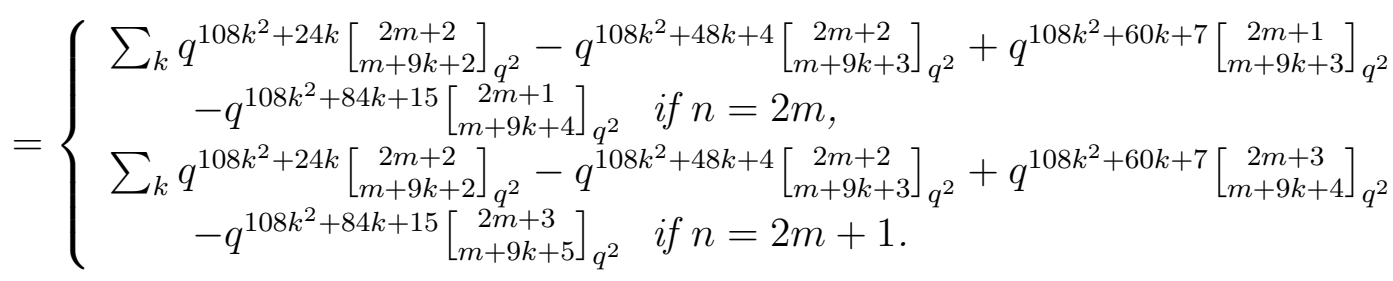

$$
\begin{aligned}
& P_{0}=1 \\
& P_{1}=1 \\
& P_{2}=q^{6}+q^{2}+1 \\
& P_{3}=q^{7}+q^{6}+q^{2}+1 \\
& P_{n}=\left(1-q^{2}\right) P_{n-1}+\left(2 q^{2}+q^{2 n+2}\right) P_{n-2}+\left(-q^{2}+q^{4}+q^{2 n+1}\right) P_{n-3} \\
& +\left(q^{2 n}-q^{4}\right) P_{n-4} \quad \text { if } n \geqq 4
\end{aligned}
$$

Observation 3.126. Identity (126) is equivalent to $(71)+q \times(68)-q \times(128)$. 
Observation 3.127. Identity (127) is equivalent to $(71)-q \times(128)$.

Identity 3.128 (Finite form of A.128).

$$
\begin{gathered}
\sum_{j \geqq 0} \sum_{i \geqq 0} \sum_{I \geqq 0} \sum_{k \geqq 0} \sum_{K \geqq 0} \sum_{L \geqq 0}(-1)^{i+L} q^{j^{2}+2 j+2 i^{2}+2 i+2 I^{2}+2 I+4 k+K+2 L}\left[\begin{array}{l}
j \\
i
\end{array}\right]_{q^{4}}\left[\begin{array}{l}
j \\
I
\end{array}\right]_{q^{4}}\left[\begin{array}{c}
j+k \\
k
\end{array}\right]_{q^{4}} \\
\times\left[\begin{array}{c}
j+K \\
K
\end{array}\right]_{q^{2}}\left[\begin{array}{c}
j+L-1 \\
L
\end{array}\right]_{q^{2}}\left[\begin{array}{c}
n-2 i-2 I-2 k-K+L \\
j
\end{array}\right]_{q^{2}} \\
=\sum_{k=-\infty}^{\infty} q^{32 k^{2}+12 k} \mathrm{U}(n+1,8 k+1 ; q)-q^{32 k^{2}+28 k+5} \mathrm{U}(n+1,8 k+3 ; q) \\
P_{1}=q^{3}+q+1 \\
P_{2}=q^{8}+q^{6}+2 q^{4}+q^{3}+q^{2}+q+1 \\
P_{n}=\left(1+q+q^{2 n+1}\right) P_{n-1}+\left(q^{4}-q-q^{2 n+1}\right) P_{n-2}+\left(-q^{5}-q^{4}+q^{2 n+1}\right) P_{n-3} \\
+\left(q^{5}-q^{2 n+1}\right) P_{n-4} \text { if } n \geqq 4 .
\end{gathered}
$$

Observation 3.129. Identity (129) is equivalent to $q^{-2} \times((128)-(68))$.

Identity 3.130 (Finite form of A.130).

$$
\begin{gathered}
\sum_{i \geqq 0} \sum_{j \geqq 0} \sum_{k \geqq 0} \sum_{l \geqq 0}(-1)^{l} q^{j^{2}+2 i^{2}+k+2 l}\left[\begin{array}{l}
j \\
i
\end{array}\right]_{q^{4}}\left[\begin{array}{c}
j+k \\
k
\end{array}\right]_{q^{2}}\left[\begin{array}{c}
j+l-1 \\
l
\end{array}\right]_{q^{2}}\left[\begin{array}{c}
n-2 i-k-l]_{q^{2}} \\
j
\end{array}\right]_{j=-\infty}^{\infty}(-1)^{j} q^{8 j^{2}}\left[\mathrm{~T}_{0}(n, 4 j ; q)+\mathrm{T}_{0}(n-1,4 j ; q)\right] \\
+(-1)^{j} q^{8 j^{2}+4 j+1}\left[\mathrm{~T}_{0}(n, 4 j+1 ; q)+\mathrm{T}_{0}(n-1,4 j+1 ; q)\right] \\
P_{0}=1 \\
P_{1}=2 q+1 \\
P_{2}=2 q^{4}+2 q^{2}+2 q+1 \\
P_{n}=\left(1+q-q^{2}+q^{2 n-1}\right) P_{n-1}+\left(q^{3}+q^{2}-q\right) P_{n-2}+\left(q^{2 n-3}-q^{3}\right) P_{n-3} \quad \text { if } n \geqq 3
\end{gathered}
$$

\section{Proving Conjectured Polynomial Identities}

We shall now discuss several methods by which one can prove the identities stated in the previous section. 


\subsection{Direct Proof}

The following finitization of identity 39 on Slater's list

$$
\sum_{j \geqq 0} q^{2 j^{2}}\left[\begin{array}{c}
n \\
2 j
\end{array}\right]_{q}=\sum_{j=-\infty}^{\infty} q^{4 j^{2}+j}\left[\begin{array}{c}
n \\
\left\lfloor\frac{n+4 j+1}{2}\right\rfloor
\end{array}\right]_{q^{2}}
$$

was stated by Andrews and Santos in [20, p. 94, eqn. 3.1], but only hint of the proof was given, since it is a finite analog of the proof of (A.39), which was written out in full. The details of the proof hinted at are presented below:

Proof.

$$
\begin{aligned}
& \sum_{j \geqq 0} q^{2 j^{2}}\left[\begin{array}{c}
n \\
2 j
\end{array}\right]_{q} \\
= & \sum_{j \geqq 0} q^{(2 j)^{2} / 2}\left[\begin{array}{c}
n \\
2 j
\end{array}\right]_{q} \\
= & \sum_{k \geqq 0} q^{k^{2} / 2}\left[\begin{array}{l}
n \\
k
\end{array}\right]_{q} \\
= & \frac{1}{2}\left(\sum_{k \geqq 0} q^{k^{2} / 2}\left[\begin{array}{l}
n \\
k
\end{array}\right]_{q}+\sum_{k \geqq 0}(-1)^{k} q^{k^{2} / 2}\left[\begin{array}{l}
n \\
k
\end{array}\right]_{q}\right) \\
= & \frac{1}{2}\left[(-\sqrt{q} ; q)_{n}+(\sqrt{q} ; q)_{n}\right] \quad(\text { by }(1.10))
\end{aligned}
$$

CASE 1: (Even $n)$

$$
\begin{aligned}
& =\frac{1}{2}\left[\left(-\sqrt{q} ; q^{2}\right)_{n / 2}\left(-\sqrt{q^{3}} ; q^{2}\right)_{n / 2}+\left(\sqrt{q} ; q^{2}\right)_{n / 2}\left(\sqrt{q^{3}} ; q^{2}\right)_{n / 2}\right] \\
& =\frac{1}{2}\left(\sum_{k=-\infty}^{\infty} q^{k^{2}+k / 2}\left[\begin{array}{c}
n \\
n / 2+k
\end{array}\right]_{q^{2}}+\sum_{k=-\infty}^{\infty}(-1)^{k} q^{k^{2}+k / 2}\left[\begin{array}{c}
n \\
n / 2+k
\end{array}\right]_{q^{2}}\right) \\
& =\sum_{k=-\infty}^{\infty} q^{k^{2}+k / 2}\left[\begin{array}{c}
n \\
n / 2+k
\end{array}\right]_{q^{2}} \\
& =\sum_{j=-\infty}^{\infty} q^{4 j^{2}+j}\left[\begin{array}{c}
n \\
\left\lfloor\frac{n+4 j}{2}\right\rfloor
\end{array}\right]_{q^{2}} \\
& =\sum_{j=-\infty}^{\infty} q^{4 j^{2}+j}\left[\begin{array}{c}
n \\
\left\lfloor\frac{n+4 j+1}{2}\right\rfloor
\end{array}\right]_{q^{2}}
\end{aligned}
$$


CASE 2: $(\operatorname{Odd} n)$

$$
\begin{aligned}
& =\frac{1}{2}\left[\left(-\sqrt{q} ; q^{2}\right)_{(n+1) / 2}\left(-\sqrt{q^{3}} ; q^{2}\right)_{(n-1) / 2}+\left(\sqrt{q} ; q^{2}\right)_{(n+1) / 2}\left(\sqrt{q^{3}} ; q^{2}\right)_{(n-1) / 2}\right] \\
& =\frac{1}{2}\left[\left(1+q^{(2 n-1) / 2}\right)\left(-\sqrt{q} ; q^{2}\right)_{(n-1) / 2}\left(-\sqrt{q^{3}} ; q^{2}\right)_{(n-1) / 2}\right. \\
& \left.+\left(1-q^{(2 n-1) / 2}\right)\left(\sqrt{q} ; q^{2}\right)_{(n-1) / 2}\left(\sqrt{q^{3}} ; q^{2}\right)_{(n-1) / 2}\right] \\
& =\frac{1}{2}\left(\sum_{k=-\infty}^{\infty} q^{k^{2}+k / 2}\left[\begin{array}{c}
n-1 \\
(n-1) / 2+k
\end{array}\right]_{q^{2}}+\sum_{k=-\infty}^{\infty}(-1)^{k} q^{k^{2}+k / 2}\left[\begin{array}{c}
n-1 \\
(n-1) / 2+k
\end{array}\right]_{q^{2}}\right) \\
& +\frac{q^{(2 n-1) / 2}}{2}\left(\sum_{k=-\infty}^{\infty} q^{k^{2}+k / 2}\left[\begin{array}{c}
n-1 \\
(n-1) / 2+k
\end{array}\right]_{q^{2}}\right. \\
& \left.+\sum_{k=-\infty}^{\infty}(-1)^{k} q^{k^{2}+k / 2}\left[\begin{array}{c}
n-1 \\
(n-1) / 2+k
\end{array}\right]_{q^{2}}\right) \\
& =\sum_{\substack{k=-\infty \\
k \text { even }}}^{\infty} q^{k^{2}+k / 2}\left[\begin{array}{c}
n-1 \\
(n-1) / 2+k
\end{array}\right]_{q^{2}}+\sum_{\substack{k=-\infty \\
k \text { odd }}}^{\infty} q^{k^{2}+k / 2}\left[\begin{array}{c}
n-1 \\
(n-1) / 2+k
\end{array}\right]_{q^{2}} \\
& =\sum_{j=-\infty}^{\infty} q^{4 j^{2}-j}\left[\begin{array}{c}
n-1 \\
(n-1) / 2-2 j
\end{array}\right]_{q^{2}} \\
& +q^{n-1 / 2} \sum_{j=-\infty}^{\infty} q^{(1-2 j)^{2}+(1-2 j) / 2}\left[\begin{array}{c}
n-1 \\
(n-1) / 2-2 j+1
\end{array}\right]_{q^{2}} \\
& =\sum_{j=-\infty}^{\infty} q^{4 j^{2}-j}\left[\begin{array}{c}
n-1 \\
(n-1) / 2-2 j
\end{array}\right]_{q^{2}}+\sum_{j=-\infty}^{\infty} q^{n+1-4 j} q^{4 j^{2}-j}\left[\begin{array}{c}
n-1 \\
\frac{n+1-4 j}{2}
\end{array}\right]_{q^{2}} \\
& =\sum_{j=-\infty}^{\infty} q^{4 j^{2}-j}\left(\left[\begin{array}{c}
n-1 \\
\frac{n+1-4 j}{2}-1
\end{array}\right]_{q^{2}}+q^{n+1-4 j}\left[\begin{array}{c}
n-1 \\
\frac{n+1-4 j}{2}-1
\end{array}\right]_{q^{2}}\right) \\
& =\sum_{j=-\infty}^{\infty} q^{4 j^{2}-j}\left[\begin{array}{c}
n \\
\frac{n+1-4 j}{2}
\end{array}\right]_{q^{2}} \quad(\text { by }(1.6)) \\
& =\sum_{j=-\infty}^{\infty} q^{4 j^{2}+j}\left[\begin{array}{c}
n \\
\frac{n+1+4 j}{2}
\end{array}\right]_{q^{2}} \\
& =\sum_{j=-\infty}^{\infty} q^{4 j^{2}+j}\left[\begin{array}{c}
n \\
\left\lfloor\frac{n+1+4 j}{2}\right\rfloor
\end{array}\right]_{q^{2}}
\end{aligned}
$$

While the preceeding proof is nice in that it is a finite analog of the proof of (A.39) given by Andrews and Santos [20, pp. 93-94], the method is not applicable to all the polynomial identities stated in $\S 3$. 


\subsection{WZ proofs}

We now turn our attention to a method which, in theory, will provide proofs for all of the polynomial identities in $\S 3$. In 1990, Wilf and Zeilberger published their groundbreaking paper "Rational Functions Certify Combinatorial Identities" [76], for which they later won the prestigious Leroy P. Steele Prize for Seminal Contribution to Research [2]. Their original methods, which were designed for single sum hypergeometric type identities, were successfully extended to multisum identities and $q$-analogs (see, e.g. Wilf and Zeilberger [78] and Koornwinder [47]).

\subsubsection{A Brief Introduction to the WZ-theory}

The following is a brief exposition of the part of Wilf and Zeilberger's WZ-theory ${ }^{4}$ which will be required for our present purposes. For a detailed introduction to WZ theory, including the Sister Celine's algorithm, Gosper's Algorithm, Zeilberger's algorithm, and WZ pairs, see Petkovšek, Wilf and Zeilberger [55].

Recall that the identities conjectured in $\S 3$ are of the form

$$
\operatorname{LHS}(n)=R H S(n)
$$

where the LHS is a polynomial known to satisfy a certain recurrence relation of order, say $r$. Thus, to prove the identity is true for all $n$, it is sufficient to show that $R H S(n)$ satisfies the same $r$ th order recurrence relation and the initial conditions

$$
\operatorname{LHS}(0)=R H S(0), \quad \operatorname{LHS}(1)=R H S(1), \ldots, \operatorname{LHS}(r-1)=R H S(r-1) .
$$

We need to introduce some notation at this point. Let us say that the $r$ th order recurrence relation satisfied by $L H S(n)$ is

$$
\sum_{i=0}^{r} p_{i}(q) L H S(n-i)=0
$$

where the $p_{i}(q)$ are polynomials in $q$ depending only on $n$, and note that $R H S(n)$ is of the form $\sum_{j=-\infty}^{\infty} F(n, j)$. We obtain a function $G(n, j)$ which satisfies the following conditions:

$$
\sum_{i=0}^{r} p_{i}(q) F(n-i, j)=G(n, j)-G(n, j-1)
$$

and

$$
\lim _{j= \pm \infty} G(n, j)=0 .
$$

Then, by summing (4.2) over all $j$, we automatically obtain

$$
\sum_{i=0}^{r} p_{i}(q) R H S(n-i)=0,
$$

\footnotetext{
${ }^{4}$ named in honor of two famous complex variables; see [76, p. 148, footnote 1].
} 
and this observation together with the checking of the appropriate initial conditions, completes the proof. Of course, the big question is, "How does one obtain this $G(n, j)$ function?" The process by which one finds $G$ is known as either "Zeilberger's Algorithm" or "creative telescoping"; see Zeilberger [80] and [79]. For a detailed explaination of why such a $G$ is guaranteed to exist, and how to find one, the reader is referred to Petkovšek, Wilf and Zeilberger [55]. For our present purposes, we merely note that there are several packages available which will find $G(n, j)$ for a given $F(n, j)$. Zeilberger's qEKHAD package ${ }^{5}$ for Maple is available for free download from his web site http://www.math.rutgers.edu/ ${ }^{\sim}$ zeilberg. Axel Riese has written Mathematica packages for proving both single and multisum $q$-hypergeometric identities. They are available for download, free of charge to researchers and non-commercial users, at http://www.risc.uni-linz.ac.at/research/combinat/risc/software/

and have accompanying documentation in Paule and Riese [54] and Riese [56] respectively. We note that the $G(n, j)$ function is a rational function multiple of the summand function $F(n, j)$, i.e. that

$$
G(n, j)=F(n, j) R(n, j)
$$

for some rational function $R(n, j)$. This function $R(n, j)$ is called the $W Z$ certificate or simply certificate function, and it is actually this certificate function rather than the $G(n, j)$ function that is produced for a given summand $F(n, j)$ by the Maple and Mathematica packages of Zeilberger and Riese respectively.

\section{A Detailed Example.}

Suppose we are interested in proving the identity conjectured in $\S 2$ using Zeilberger's Algorithm. Recall that the identity is

$$
\sum_{j=0}^{n} q^{j^{2}+j}\left[\begin{array}{l}
n \\
j
\end{array}\right]_{q^{2}}=\sum_{j=-\infty}^{\infty}(-1)^{j} q^{2 j^{2}+j}\left[\begin{array}{c}
2 n+1 \\
n+2 j+1
\end{array}\right]_{q} .
$$

We saw in $\S 2$ that the idenitity (4.4) satisfies the recurrence

$$
P_{n}(q)=\left(1+q^{2 n}\right) P_{n-1}(q)
$$

and the initial condition $P_{0}(q)=1$. Thus to prove (4.4), it suffices to show that the righthand side of (4.4) satisfies, the same recurrence and initial conditions, i.e. that

$$
\sum_{j=-\infty}^{\infty} F(n, j)=\left(1+q^{2 n}\right) \sum_{j=-\infty}^{\infty} F(n-1, j),
$$

for $n \geqq 0$ and

$$
\sum_{j=-\infty}^{\infty} F(0, j)=1
$$

\footnotetext{
${ }^{5}$ named in honor of Shalosh B. Ekhad, Zeilberger's computer and prolific author in its own right.
} 
where $F(n, j)=(-1)^{j} q^{2 j^{2}+j}\left[\begin{array}{c}2 n+1 \\ n+2 j+1\end{array}\right]_{q}$. As mentioned in the previous section, one can use the qZeil function in Riese's qZeil Mathematica package to produce a function $G(n, j)$ such that

$$
F(n, j)-\left(1+q^{2 n}\right) F(n-1, j)=G(n, j)-G(n, j-1)
$$

and

$$
\lim _{j \rightarrow \pm \infty} G(n, j)=0 .
$$

We then sum both sides of equation (4.7) over all integral $j$, and observe that the righthand side of (4.7) telescopes to 0. This will then guarantee that equation (4.5) holds. Let us now begin a Mathematica session (for a detailed explanation of the use of the qZeil package, see Paule and Riese [54]):

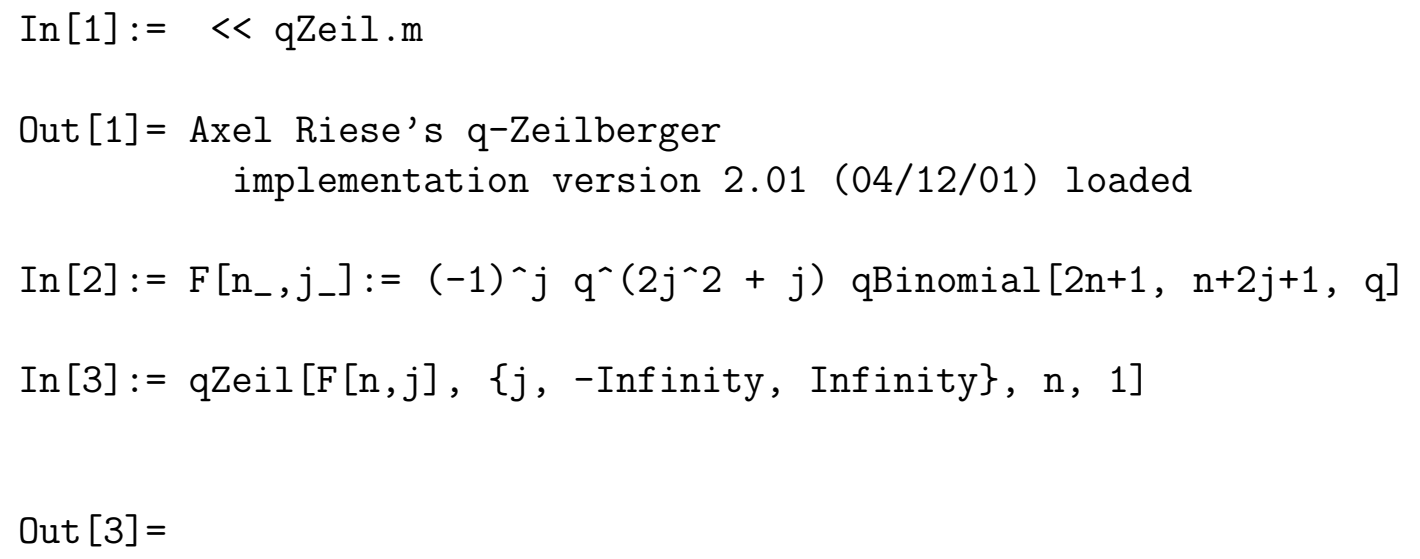

$$
S U M(n)=\left(1+q^{2 n}\right) S U M(-1+n)
$$

I interrupt the Mathematica session here to note that if we trust the computer, the proof of the identity is complete, since the qZeil procedure output the recurrence we are trying to demonstrate that $\sum F(n, j)$ satisfies. (Remember that the initial conditions were already verified in an earlier Maple session. However, if we do not implicitly trust the computer, we can use it to produce the WZ certificate, which in turn allows us to verify a rational function identity algebraically equivalent to (4.7). Let us therefore continue the Mathematica session by producing the WZ certificate ${ }^{6}$ :

$\operatorname{In}[4]:=\operatorname{Cert}[]$

Out $[4]=$

$$
\frac{q^{-2 j+n}\left(-q^{2 j}+q^{n}\right)\left(-q^{1+2 j}+q^{n}\right)}{\left(-1+q^{n}\right)\left(1+q^{n}\right)\left(-1+q^{1+2 n}\right)}
$$

\footnotetext{
${ }^{6}$ Note that Zeilberger's algorithm is much stronger than we actually need here. Zeilberger's algorithm not only finds the certificate, but the recurrence satisfied by the summand. Thus, even though the method of $q$-difference equations allows us to know the recurrence in advance, Zeilberger's algorithm by no means requires this.
} 
Thus, the qZeil package claims that

$$
G(n, j)=F(n, j) R(n, j)
$$

where

$$
R(n, j)=\frac{q^{n-2 j}\left(q^{n}-q^{2 j}\right)\left(q^{n}-q^{2 j+1}\right)}{\left(q^{n}-1\right)\left(q^{n}+1\right)\left(q^{2 n+1}-1\right)}
$$

is the desired function $G$. It is easy to see that $G(n, j)$ satifies equation (4.8) since the Gaussian polynomial $\left[\begin{array}{l}A \\ B\end{array}\right]_{q}$ is 0 when $B>A$ and when $B<0$. We now must verify that it satisfies equation (4.7). For this purpose, it is easiest to divide (4.7) through by $F(n, j)$ to obtain the equivalent formulation

$$
1-\frac{\left(1+q^{2 n}\right) F(n-1, j)}{F(n, j)}=R(n, j)-\frac{F(n, j-1) R(n, j-1)}{F(n, j)} .
$$

Continuing with Mathematica:

$$
\begin{aligned}
& \operatorname{In}[5]:=R\left[n_{-}, j_{-}\right]:=\left(q^{\wedge}(n-2 j)\left(-q^{\wedge}(2 j)+q^{\wedge} n\right)\left(q^{\wedge} n-q^{\wedge}(2 j+1)\right)\right) / \\
& \qquad\left(\left(q^{\wedge} n-1\right)\left(q^{\wedge} n+1\right)\left(q^{\wedge}(2 n+1)-1\right)\right) \\
& \operatorname{In}[6]:=\text { Ihs = qSimplify }\left[1-\left(1+q^{\wedge}(2 n)\right) F[n-1, j] / F[n, j]\right] \\
& \text { Out }[6]=
\end{aligned}
$$

$$
1+\frac{\left(-1-q^{2 n}\right)\left(1-q^{-2 j+n}\right)\left(1-q^{1+2 j+n}\right)}{\left(1-q^{2 n}\right)\left(1-q^{1+2 n}\right)}
$$

$\operatorname{In}[7]:=\operatorname{rhs}=\operatorname{qSimplify}[R[n, j]-F[n, j-1] R[n, j-1] / F[n, j]]$

Out $[7]=$

$$
\begin{gathered}
\frac{q^{-2 j+n}\left(-q^{2 j}+q^{n}\right)\left(-q^{1+2 j}+q^{n}\right)}{\left(1-q^{2 n}\right)\left(1-q^{1+2 n}\right)}+ \\
\frac{q^{3-6 j+n}\left(-q^{-2+2 j}+q^{n}\right)\left(-q^{-1+2 j}+q^{n}\right)\left(1-q^{2 j+n}\right)\left(1-q^{1+2 j+n}\right)}{\left(1-q^{2 n}\right)\left(1-q^{1-2 j+n}\right)\left(1-q^{2-2 j+n}\right)\left(1-q^{1+2 n}\right)}
\end{gathered}
$$

In $[8]:=$ Simplify [lhs - rhs]

Out $[8]=0$

And thus using the Mathematica Simplify procedure together with the qSimplify procedure available in the qZeil package, we see that (4.9), and therefore (4.7), and therefore (4.5) is true. Of course, the complete computer skeptic can verify (4.9) directly by hand. 


\subsubsection{Dealing with Non-minimal Recurrences}

Paule's Creative Symmetrization. It is well known (see e.g. Paule [53]) that creative telescoping does not always output the minimal recurrence that is satisfied by the summand. Let us examine an example where this is the case, Identity 3.5-b:

$$
\sum_{j \geqq 0} q^{2 j^{2}+j}\left[\begin{array}{c}
n+1 \\
2 j+1
\end{array}\right]_{q}=\sum_{j=-\infty}^{\infty}(-1)^{j} q^{3 j^{2}+j}\left[\begin{array}{c}
2 n \\
n+2 j
\end{array}\right]_{q}
$$

From the Maple session in which this identity was conjectured we know that the LHS satisfies the recurrence

$$
\begin{gathered}
P_{0}=1, \\
P_{n}=\left(1+q^{n}\right) P_{n-1} \text { if } n \geqq 1 .
\end{gathered}
$$

We return to Mathematica in the hopes of demonstrating that the RHS satisfies the same recurrence:

$$
\begin{aligned}
\operatorname{In}[9]:= & \text { qZeil }\left[(-1)^{\wedge} j \mathrm{q}^{\wedge}\left(3 j^{\wedge} 2+j\right) q \operatorname{qBinomial}[2 \mathrm{n}, \mathrm{n}+2 j, \mathrm{q}],\right. \\
& \{j,- \text { Infinity, Infinity }\}, \mathrm{n}, 3]
\end{aligned}
$$

Out $[9]=$

$$
\begin{gathered}
S U M(n)=q^{3}\left(1-q^{-5+2 n}\right)\left(1-q^{-4+2 n}\right) S U M(-3+n)- \\
\frac{\left(q^{5}+q^{6}+q^{7}+q^{3 n}-q^{2+2 n}+q^{2+3 n}\right) S U M(-2+n)}{q^{4}}- \\
\frac{\left(-q-q^{2}-q^{3}-q^{2 n}\right) S U M(-1+n)}{q}
\end{gathered}
$$

but alas the creative telescoping algorithm finds a third order recurrence, when we were attempting to show that the RHS satisfies a certain first order recurrence. But thanks to the following useful observation of Peter Paule, all hope is not lost.

Notice that any function $F(n, j)$ can be written as the sum of its even part and its odd part, i.e.

$$
F(n, j)=\frac{F(n, j)+F(n,-j)}{2}+\frac{F(n, j)-F(n,-j)}{2} .
$$

If we sum both sides of (4.10) over all $j \in \mathbb{Z}$, the odd portion of $F(n, j)$ vanishes, so we obtain

$$
\sum_{j \in \mathbb{Z}} F(n, j)=\sum_{j \in \mathbb{Z}} \frac{F(n, j)+F(n,-j)}{2}
$$

Applying (4.11) to the RHS of (3.5), we obtain

$$
\sum_{j=-\infty}^{\infty} 2 F(n, j)=\sum_{j=-\infty}^{\infty}(-1)^{j} q^{3 j^{2}-j}\left[\begin{array}{c}
2 n \\
n+2 j
\end{array}\right]_{q}\left(1+q^{2 j}\right)
$$


In[10]:= qZeil[ $(-1)^{\wedge} j q^{\wedge}\left(3 j^{\wedge} 2-j\right)\left(1+q^{\wedge}(2 j)\right)$ qBinomial $[2 n, n+2 j$,

$\mathrm{q}],\{j,-$ Infinity, Infinity $\}, \mathrm{n}, 1]$

Out $[10]=$

$$
\operatorname{SUM}(n)=\left(1+q^{n}\right) S U M(-1+n)
$$

which is the desired (minimal) recurrence.

Operator Algebra. The technique of creative symmetrization works perfectly on the example under consideration and in fact works on many well known examples (see Paule [54]). However, creative symmetrization by no means guarantees that the minimal, or even a lower order recurrence, will be produced using Zeilberger's algorithm. It would therefore be wise to have a way of dealing with non-minimal recurrences in the event that creative symmetrizing fails. For comparison, I will continue to work with the same example as from the previous section, even though the creative symmetrizing technique produces a minimal recurrence for it. But first, the forward shift operator must be introduced. Define

$$
N F(n, j):=F(n+1, j)
$$

and by extension,

$$
N^{s} F(n, j):=F(n+s, j)
$$

for any integer $s$. For example, (4.7) re-written in operator notation is

$$
\left(1-\left(1+q^{2 n}\right) N^{-1}\right) F(n, j)=\left(1-J^{-1}\right) G(n, j),
$$

where, of course, $J$ is the forward shift operator in $j$, i.e.

$$
J F(n, j):=F(n, j+1)
$$

and by extension,

$$
J^{s} F(n, j):=F(n, j+s)
$$

for any integer $s$.

Now the (unsymmetrized) RHS of (3.5-b) was shown by Mathematica to satisfy the recurrence

$$
\begin{gathered}
\sum_{j} F(n)=q^{3}\left(1-q^{2 n-5}\right)\left(1-q^{2 n-4}\right) \sum_{j} F(n-3, j) \\
-\left(q+q^{2}+q^{3}+q^{3 n}-q^{2 n-2}+q^{3 n-2}\right) \sum_{j} F(n-2, j) \\
+\left(1+q+q^{2}+q^{2 n-1}\right) \sum_{j} F(n-1, j),
\end{gathered}
$$


which is easily seen to be equivalent to

$$
\begin{gathered}
-q^{3}\left(1-q^{2 n+1}\right)\left(1-q^{2 n+2}\right) \sum_{j} F(n, j) \\
+\left(q+q^{2}+q^{3}-q^{2 n+4}+q^{3 n+5}+q^{3 n+7}\right) \sum_{j} F(n+1, j) \\
-\left(1+q+q^{2}+q^{2 n+5}\right) \sum_{j} F(n+2, j) \\
+\sum_{j} F(n+3, j)=0,
\end{gathered}
$$

which in turn can be written in operator notation as

$$
\begin{gathered}
{\left[-q^{3}\left(1-q^{2 n+1}\right)\left(1-q^{2 n+2}\right)+\left(q+q^{2}+q^{3}-q^{2 n+4}+q^{3 n+5}+q^{3 n+7}\right) N\right.} \\
\left.-\left(1+q+q^{2}+q^{2 n+5}\right) N^{2}+N^{3}\right] \sum_{j} F(n, j)=0 .
\end{gathered}
$$

We say that an operator $B$ annihilates a sequence $S(n)$ if

$$
[B] S(n)=0,
$$

so it is clear that the operator in (4.14) annihilates the RHS of (3.5-b). Similarly, the LHS of (3.5-b) was shown by Mathemtaica to satisfy the recurrence

$$
\sum_{j} F(n, j)=\left(1+q^{n}\right) \sum_{j} F(n-1, j)
$$

which is equivalent to the recurrence

$$
\sum_{j} F(n+1, j)-\left(1+q^{n+1}\right) \sum_{j} F(n, j)=0
$$

which in turn can be written in operator notation as

$$
\left[N-\left(1+q^{n+1}\right)\right] \sum_{j} F(n, j)=0 .
$$

Now it is clear that if an operator $B$ annihilates a sequence $S(n)$, then any left multiple of $B$ will also annihilate $S(n)$, i.e.

$$
[B] S(n)=0 \Longrightarrow[A B] S(n)=0
$$

where $A$ is any $n$-shift operator. Thus, if it can be shown that the operator in (4.14) is a left multiple of (4.15) (along with the appropriate initial conditions), then the identity will 
be proved. Dividing the third order recurrence on the right by the first order recurrence, we find that

$$
\begin{gathered}
{\left[N^{2}-q\left(1+q-q^{n+2}+q^{2 n+4}\right) N+q^{3}\left(q^{2 n+1}-1\right)\left(q^{n+1}-1\right)\right]\left[N-\left(1+q^{n+1}\right)\right]} \\
=\left[-q^{3}\left(1-q^{2 n+1}\right)\left(1-q^{2 n+2}\right)+\left(q+q^{2}+q^{3}-q^{2 n+4}+q^{3 n+5}+q^{3 n+7}\right) N\right. \\
\left.-\left(1+q+q^{2}+q^{2 n+5}\right) N^{2}+N^{3}\right]
\end{gathered}
$$

and so both the LHS and RHS of (3.5-b) are annhilated by the third order recurrence found by Mathematica, and together with the appropriate initial conditions, the identity is established.

\subsubsection{Multisum techniques}

The Bosonic forms for each of the identities in $\S 3$ are single sums, but many are single sums involving $q$-trinomial coëfficients. There are currently no computer implementations of Zeilberger's algorithm which allows direct inputting of $q$-trinomial coëfficients, but recalling the definitions of the $q$-trinomial coëfficients (1.16-1.22), we can express any single sum $q$-trinomial expressions as a double sum $q$-binomial expression. Thus the situation we are now in is wanting to prove an identity

$$
L H S(n)=R H S(n)
$$

where $R H S(n)$ is of the form

$$
R H S(n)=\sum_{j=-\infty}^{\infty} \sum_{s=0}^{\infty} F(n, j, s)
$$

for all nonnegative integers $n$. The conditions analogous to (4.2) and (4.3) in the two-fold sum case is that we find functions $G(n, j, s)$ and $H(n, j, s)$ such that

$$
\sum_{i=0}^{r} p_{i}(q) F(n+i, j, s)=G(n, j, s)-G(n, j-1, s)+H(n, j, s)-H(n, j, s-1)
$$

and

$$
\lim _{j \rightarrow \pm \infty} G(n, j, s)=\lim _{s \rightarrow \pm \infty} H(n, j, s)=0
$$

Then, by summing (4.16) over all $j$ and $s$, we immediately obtain

$$
\sum_{i=0}^{r} p_{i}(q) R H S(n-i)=0
$$

which, together with the appropriate initial conditions, will complete the proof. The WZ certificate is now a pair of functions $R_{j}(n, j, s)$ and $R_{s}(n, j, s)$ such that

$$
G(n, j, s)=F(n, j, s) R_{j}(n, j, s)
$$


and

$$
H(n, j, s)=F(n, j, s) R_{s}(n, j, s) .
$$

(Of course, these ideas extend from two-fold summation to $k$-fold summation for any fixed positive integer $k$.)

\section{A Detailed Example.}

Let us consider the identity (3.4).

$$
\begin{gathered}
\sum_{i \geqq 0} \sum_{j \geqq 0} \sum_{k \geqq 0}(-1)^{j+k} q^{i^{2}+j^{2}+2 k}\left[\begin{array}{l}
j \\
i
\end{array}\right]_{q^{2}}\left[\begin{array}{c}
j+k-1 \\
k
\end{array}\right]_{q^{2}}\left[\begin{array}{c}
n-i-k \\
j
\end{array}\right]_{q^{2}} \\
=\sum_{j=-\infty}^{\infty}(-1)^{j} q^{j^{2}} \mathrm{U}(n-1, j ; q)
\end{gathered}
$$

where during the Maple session in which it was conjectured, the LHS was found to satisfy the recurrence

$$
\begin{gathered}
P_{0}=1 \\
P_{1}=-q+1, \\
P_{n}=\left(1-q^{2}-q^{2 n-1}\right) P_{n-1}+\left(q^{2}-q^{2 n-2}\right) P_{n-2} \text { if } n \geqq 2 .
\end{gathered}
$$

Translating the above into forward shifts and operator notation, we obtain

$$
\left[-q^{2}\left(-1+q^{n}\right)\left(1+q^{n}\right)+\left(1-q^{2}-q^{3 n+3}\right) N-N^{2}\right] \sum \sum F(n, j, r)=0
$$

Thus, it needs to be shown that the RHS satisfies the same recurrence (or an operator algebraic multiple of it) and the initial conditions. Now as stated previously, the Riese Mathematica packages can not deal with the RHS of (3.4) directly. Instead, we must use (1.22) and (1.17) to find that

$$
\begin{gathered}
\sum_{j=-\infty}^{\infty}(-1)^{j} q^{j^{2}} \mathrm{U}(n-1, j ; q)=\sum_{j=-\infty}^{\infty}(-1)^{j} q^{j^{2}}\left\{\mathrm{~T}_{0}(n-1, j ; q)+\mathrm{T}_{0}(n-1, j+1 ; q)\right\} \\
=\sum_{j=-\infty}^{\infty}(-1)^{j} q^{j^{2}}\left\{\sum_{r=0}^{n-1}(-1)^{r}\left[\begin{array}{c}
n-1 \\
r
\end{array}\right]_{q^{2}}\left[\begin{array}{c}
2 n-2-2 r \\
n-1-j-r
\end{array}\right]_{q}\right. \\
\left.+\sum_{r=0}^{n}(-1)^{r}\left[\begin{array}{c}
n-1 \\
r
\end{array}\right]_{q^{2}}\left[\begin{array}{c}
2 n-2-2 r \\
n-j-r
\end{array}\right]_{q}\right\} \\
=\sum_{j=-\infty}^{\infty} \sum_{r=0}^{\infty}(-1)^{j+r} q^{j^{2}}\left[\begin{array}{c}
n-1 \\
r
\end{array}\right]_{q^{2}}\left(\left[\begin{array}{c}
2 n-2 r-2 \\
n-j-r-1
\end{array}\right]_{q}+\left[\begin{array}{c}
2 n-2 r-2 \\
n-j-r
\end{array}\right]_{q}\right) \\
=\sum_{j=-\infty}^{\infty} \sum_{r=0}^{\infty}(-1)^{j+r} q^{j^{2}}\left[\begin{array}{c}
n-1 \\
r
\end{array}\right]_{q^{2}}\left[\begin{array}{c}
2 n-2 r-2 \\
n-j-r-1
\end{array}\right]_{q}\left(1+\frac{1-q^{n+j-r-1}}{1-q^{n-j-r}}\right) .
\end{gathered}
$$

We now return to Mathematica, this time using Riese's qMultiSum package. 


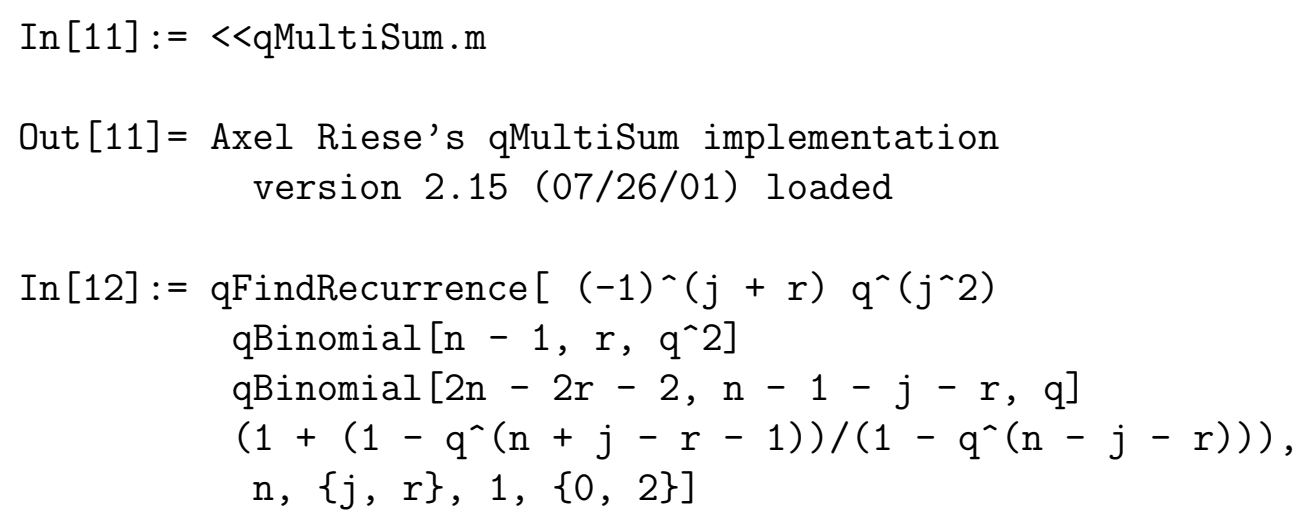

Out $[12]=$

$$
\begin{gathered}
-\left(\left(q^{2}-q^{n}\right)\left(q^{3}-q^{n}\right)\left(q^{4}-q^{n}\right)\left(q^{2}+q^{n}\right)\left(q^{3}+q^{n}\right)\left(q^{4}+q^{n}\right)\right. \\
F[-4+n,-1+j,-1+r] \\
+q^{6}\left(-1+q+q^{2}\right)\left(q^{2}-q^{n}\right)\left(q^{3}-q^{n}\right)\left(q^{2}+q^{n}\right)\left(q^{3}+q^{n}\right) \\
F[-3+n,-1+j,-1+r] \\
-q\left(q^{2}-q^{n}\right)\left(q^{3}-q^{n}\right)\left(q^{2}+q^{n}\right)\left(q^{3}+q^{n}\right)\left(q^{7}-q^{2 n}\right) F[-3+n,-1+j, r] \\
-q^{8+2 n}\left(q^{2}-q^{n}\right)\left(q^{2}+q^{n}\right) F[-2+n,-2+j, r] \\
-q^{11}\left(-1-q+q^{2}\right)\left(q^{2}-q^{n}\right)\left(q^{2}+q^{n}\right) F[-2+n,-1+j,-1+r] \\
+q^{9}\left(q^{2}-q^{n}\right)\left(q^{2}+q^{n}\right)\left(-q^{3}+q^{4}+q^{5}+q^{2 n}\right) F[-2+n,-1+j, r] \\
-q^{6+2 n}\left(q^{2}-q^{n}\right)\left(q^{2}+q^{n}\right) F[-2+n, j, r] \\
-q^{14+2 n} F[-1+n,-2+j, r]-q^{15} F[-1+n,-1+j,-1+r] \\
-q^{11}\left(-q^{4}-q^{5}+q^{6}-q^{2 n}\right) F[-1+n,-1+j, r]-q^{12+2 n} F[-1+n, j, r] \\
\left.-q^{15} F[n,-1+j, r]\right)==0
\end{gathered}
$$

Thus we have a recurrence satisfied by the RHS summand. Notice, however, that the recurrence involves shifts in the summation variables as well as in $n$. To remedy this, we use the qRecurrenceToCertificate and qSumCertificate procedures.

In [13]:= qRecurrenceToCertificate [\%]

Out $[13]=$

$$
\begin{gathered}
\Delta_{j}\left[q^{18}\left(-1+q^{n}\right)\left(1+q^{n}\right)\left(-1+q^{1+n}\right)\left(1+q^{1+n}\right)\left(-1+q^{2+n}\right)\left(1+q^{2+n}\right) F[n, j, r]\right. \\
+q^{16}\left(-1+q+q^{2}\right)\left(-1+q^{1+n}\right)\left(1+q^{1+n}\right)\left(-1+q^{2+n}\right)\left(1+q^{2+n}\right) F[1+n, j, r] \\
+q^{18}\left(-1+q^{1+n}\right)\left(1+q^{1+n}\right)\left(-1+q^{2+n}\right)\left(1+q^{2+n}\right)\left(-1+q^{1+2 n}\right) F[1+n, j, 1+r] \\
\quad+q^{15}\left(-1-q+q^{2}\right)\left(-1+q^{2+n}\right)\left(1+q^{2+n}\right) F[2+n, j, r] \\
-q^{16}\left(-1+q^{2+n}\right)\left(1+q^{2+n}\right)\left(-1+q+q^{2}-q^{2+2 n}+q^{5+2 n}\right) F[2+n, j, 1+r]
\end{gathered}
$$




$$
\begin{gathered}
+q^{18+2 n}\left(-1+q^{2+n}\right)\left(1+q^{2+n}\right) F[2+n, 1+j, 1+r] \\
-q^{15} F[3+n, j, r]-q^{15}\left(-1-q+q^{2}-q^{4+2 n}+q^{5+2 n}\right) F[3+n, j, 1+r] \\
\left.-q^{20+2 n} F[3+n, 1+j, 1+r]-q^{15} F[4+n, j, 1+r]\right] \\
+\Delta_{r}\left[q^{18}\left(-1+q^{1+n}\right)\left(1+q^{1+n}\right)\left(-1+q^{2+n}\right)\left(1+q^{2+n}\right)\left(-1+q^{1+2 n}\right) F[1+n, j, r]\right. \\
-q^{16}\left(-1+q^{2+n}\right)\left(1+q^{2+n}\right)\left(-1+q+q^{2}-q^{2+2 n}-q^{4+2 n}+q^{5+2 n}\right) F[2+n, j, r] \\
\left.-q^{15}\left(-1-q+q^{2}-q^{4+2 n}+q^{5+2 n}+q^{7+2 n}\right) F(3+n, j, r)-q^{15} F[4+n, j, r]\right] \\
+q^{18}\left(-1+q^{n}\right)\left(1+q^{n}\right)\left(-1+q^{1+n}\right)\left(1+q^{1+n}\right)\left(-1+q^{2+n}\right)\left(1+q^{2+n}\right) F[n, j, r] \\
+q^{6}\left(-1+q+q^{2}\right)\left(q^{2}-q^{4+n}\right)\left(q^{3}-q^{4+n}\right)\left(q^{2}+q^{4+n}\right)\left(q^{3}+q^{4+n}\right) F[1+n, j, r] \\
-q\left(q^{2}-q^{4+n}\right)\left(q^{3}-q^{4+n}\right)\left(q^{2}+q^{4+n}\right)\left(q^{3}+q^{4+n}\right)\left(q^{7}-q^{8+2 n}\right) F[1+n, j, r] \\
-q^{14+2 n}\left(q^{2}-q^{4+n}\right)\left(q^{2}+q^{4+n}\right) F[2+n, j, r] \\
-q^{16+2 n}\left(q^{2}-q^{4+n}\right)\left(q^{2}+q^{4+n}\right) F[2+n, j, r] \\
-q^{11}\left(-1-q+q^{2}\right)\left(q^{2}-q^{4+n}\right)\left(q^{2}+q^{4+n}\right) F[2+n, j, r] \\
+q^{9}\left(q^{2}-q^{4+n}\right)\left(q^{2}+q^{4+n}\right)\left(-q^{3}+q^{4}+q^{5}+q^{8+2 n}\right) F[2+n, j, r] \\
-q^{15} F(3+n, j, r)-q^{20+2 n} F[3+n, j, r] \\
-q^{22+2 n} F[3+n, j, r]-q^{11}\left(-q^{4}-q^{5}+q^{6}-q^{8+2 n}\right) F[3+n, j, r] \\
-q^{15} F[4+n, j, r]==0
\end{gathered}
$$

Note that the $\Delta_{j}$ and $\Delta_{r}$ are the shift operators $J-1$ and $R-1$ respectively. Thus, the functions they act on are the certificate function pair $R_{j}(n, j, r)$ and $R_{r}(n, j, r)$ respectively. We now find the recurrence (with shifts only in the $n$ ) for the RHS sum:

\section{In $[14]:=q S u m C e r t i f i c a t e[\%]$}

\section{Out $[14]=$}

$$
\begin{gathered}
q^{3}\left(-1+q^{n}\right)\left(1+q^{n}\right)\left(-1+q^{1+n}\right)\left(1+q^{1+n}\right)\left(-1+q^{2+n}\right)\left(1+q^{2+n}\right) S U M[n] \\
+q\left(-1+q^{1+n}\right)\left(1+q^{1+n}\right)\left(-1+q^{2+n}\right)\left(1+q^{2+n}\right)\left(-1+q+q^{3+2 n}\right) S U M[1+n] \\
-\left(-1+q^{2+n}\right)\left(1+q^{2+n}\right)\left(1+q^{3}-q^{3+2 n}-q^{5+2 n}+q^{6+2 n}\right) S U M[2+n] \\
-q\left(-1+q-q^{3+2 n}+q^{4+2 n}+q^{6+2 n}\right) S U M[3+n]-S U M[4+n]==0
\end{gathered}
$$

So, once again the computer finds a nonminimal recurrence. So, we perform another right division to demonstrate that the operator represented above is a left multiple of the 
operator in (4.18):

$$
\begin{gathered}
{\left[-N^{2}-(1-q)\left(-1+q^{n+2}\right)\left(1+q^{n+2}\right) N+\right.} \\
\left.q\left(1+q^{n+1}\right)\left(-1+q^{n+2}\right)\left(1+q^{n+2}\right)\left(-1+q^{n+1}\right)\right] \\
{\left[-N^{2}+\left(1-q^{2}-q^{2 n+3}\right) N+q^{2}\left(1-q^{n}\right)\left(1+q^{n}\right)\right]} \\
=N^{4}+q\left(-1+q-q^{3 n+3}+q^{2 n+4}+q^{2 n+6}\right) N^{3} \\
+\left(-1+q^{n+2}\right)\left(1+q^{n+2}\right)\left(1+q^{3}-q^{2 n+3}-q^{2 n+5}+q^{2 n+6}\right) N^{2} \\
-q\left(-1+q^{n+1}\right)\left(1+q^{n+2}\right)\left(-1+q^{n+2}\right)\left(1+q^{n+2}\right)\left(-1+q+q^{2 n+3}\right) N \\
-q^{3}\left(q^{n}-1\right)\left(1+q^{n}\right)\left(-1+q^{n+1}\right)\left(1+q^{n+1}\right)\left(-1+q^{n+2}\right)\left(1+q^{n+2}\right)
\end{gathered}
$$

\subsubsection{WZ Certificates for Selected Identities}

The RHS of Identity 3.2-b is certified by

$$
\frac{q^{-2 j+n}\left(-q^{2 j}+q^{n}\right)\left(-q^{1+2 j}+q^{n}\right)}{\left(-1+q^{n}\right)\left(1+q^{n}\right)\left(-1+q^{1+2 n}\right)} .
$$

The RHS of Identity 3.3-b is certified by

$$
\frac{q^{-2 j+n}\left(-q^{2 j}+q^{n}\right)\left(-q^{1+2 j}+q^{n}\right)}{\left(-1+q^{n}\right)\left(1+q^{n}\right)\left(-q+q^{2 n}\right)} .
$$

After creative symmetrizing with the factor $\left(1+q^{2 j}\right)$, the RHS of Identitiy 3.5-b is certified by

$$
\frac{q^{-2 j+n}\left(-q^{2 j}+q^{n}\right)\left(-q^{1+2 j}+q^{n}\right)}{\left(-1+q^{n}\right)\left(1+q^{n}\right)\left(-q+q^{2 n}\right)} .
$$

The RHS of Identity 3.11 -b is certified by

$$
\frac{q^{2 n-3 j}\left(q^{n}-q^{3 j}\right)\left(q^{n}-q^{3 j+1}\right)\left(q^{n}-q^{3 j+2}\right)}{\left(q^{n}-1\right)\left(q^{n}+1\right)\left(q^{2 n}-q\right)\left(q^{2 n+1}-1\right)} .
$$

The RHS of Identity 3.50-b is certified by

$$
\frac{q^{1-3 j+2 n}\left(-q^{3 j}+q^{n}\right)\left(-q^{1+3 j}+q^{n}\right)\left(-q^{2+3 j}+q^{n}\right)}{\left(-1+q^{n}\right)\left(1+q^{n}\right)\left(-1+q^{1+n}\right)\left(1+q^{1+n}\right)\left(-1+q^{1+2 n}\right)} .
$$

The RHS of Identity 3.96-b is certified by

$$
\begin{gathered}
-\frac{q^{2-5 k+2 n}\left(-q^{5 k}+q^{n}\right)\left(-q^{1+5 k}+q^{n}\right)\left(-q^{2+5 k}+q^{n}\right)}{\left(-1+q^{2+5 k}\right)\left(1+q^{2+5 k}\right)\left(-1+q^{n}\right)\left(1+q^{n}\right)\left(-1+q^{1+n}\right)} \\
\times \frac{\left(-q^{3+5 k}+q^{n}\right)\left(-q^{4+5 k}+q^{n}\right)}{\left(1+q^{1+n}\right)\left(-1+q^{1+2 n}\right)\left(-1+q^{3+2 n}\right)} .
\end{gathered}
$$


The RHS of Identity 3.99-b is certified by

$$
\begin{aligned}
- & \frac{q^{-5 k+2 n}\left(-q^{5 k}+q^{n}\right)\left(-q^{1+5 k}+q^{n}\right)\left(-q^{2+5 k}+q^{n}\right)\left(-q^{3+5 k}+q^{n}\right)}{\left(-1+q^{2+5 k}\right)\left(1+q^{2+5 k}\right)\left(-1+q^{n}\right)\left(1+q^{n}\right)\left(-q+q^{n}\right)\left(q+q^{n}\right)\left(-q+q^{2 n}\right)} \\
& \times \frac{\left(-q^{4+5 k}+q^{n}\right)\left(1+q^{9+10 k}-q^{3+5 k+n}-2 q^{4+5 k+n}+q^{2+5 k+3 n}\right)}{\left(-1+q^{1+2 n}\right)\left(1+q^{4+10 k}-q^{2+5 k+n}-q^{3+5 k+n}-q^{4+5 k+n}+q^{5+5 k+3 n}\right)} .
\end{aligned}
$$

\subsection{Recurrence Proof}

Depending on the complexity of the summand and recurrence, and the limits of the memory available on a given computer system, it may or may not be feasible at the present time to obtain a computer generated proof of a given finite Rogers-Ramanujan type identity. However, guided by the philosophy of the WZ method, one can show that the conjectured bosonic form satisfies the same recurrence relation and initial conditions as the known fermionic form. The main differences between this "recurrence proof" and the WZ method is that the entire summation is dealt with as a whole, rather than just the summand, and no proof certificate is produced in the process. Let us work through some examples:

Identity 3.3-T ( $q$-Trinomial Finite form of A.3/23 (with $q$ replaced by $-q)$ ).

$$
\begin{gathered}
\sum_{j \geqq 0} q^{j^{2}}\left[\begin{array}{l}
n \\
j
\end{array}\right]_{q^{2}}=\sum_{j=-\infty}^{\infty}(-1)^{j} q^{3 j^{2}+j} \mathrm{U}(n, 3 j ; q) \\
P_{0}=1, \\
P_{n}=\left(1+q^{2 n-1}\right) P_{n-1} \text { if } n \geqq 1 .
\end{gathered}
$$

Proof. Let

$$
P_{n}=\sum_{j=-\infty}^{\infty}(-1)^{j} q^{3 j^{2}+j} \mathrm{U}(n, 3 j ; q) .
$$

We need to show that $P_{n}-\left(1+q^{2 n-1}\right) P_{n-1}=0$ and $P_{0}=1$.

$$
\begin{aligned}
& P_{n}-P_{n-1}+q^{2 n-1} P_{n-1} \\
= & \sum_{j=-\infty}^{\infty}(-1)^{j} q^{3 j^{2}+j} \mathrm{U}(n, 3 j ; q)-\sum_{j=-\infty}^{\infty}(-1)^{j} q^{3 j^{2}+j} \mathrm{U}(n-1,3 j ; q) \\
& -\sum_{j=-\infty}^{\infty}(-1)^{j} q^{3 j^{2}+j+2 n-1} \mathrm{U}(n-1,3 j ; q)
\end{aligned}
$$


By applying (1.34) to the first term, we obtain

$$
\begin{aligned}
= & \sum_{j=-\infty}^{\infty}(-1)^{j} q^{3 j^{2}+j} \mathrm{U}(n-1,3 j ; q)+\sum_{j=-\infty}^{\infty}(-1)^{j} q^{3 j^{2}+j+2 n-1} \mathrm{U}(n-1,3 j ; q) \\
& +\sum_{j=-\infty}^{\infty}(-1)^{j} q^{3 j^{2}-2 j+n} \mathrm{~T}_{1}(n-1,3 j-1 ; q)+\sum_{j=-\infty}^{\infty}(-1)^{j} q^{3 j^{2}+4 j+n+1} \mathrm{~T}_{1}(n-1,3 j+2 ; q) \\
& -\sum_{j=-\infty}^{\infty}(-1)^{j} q^{3 j^{2}+j} \mathrm{U}(n-1,3 j ; q)-\sum_{j=-\infty}^{\infty}(-1)^{j} q^{3 j^{2}+j+2 n-1} \mathrm{U}(n-1,3 j ; q)
\end{aligned}
$$

whereupon the first term cancels the fifth and the second cancels the sixth leaving

$$
\begin{aligned}
& =\sum_{j=-\infty}^{\infty}(-1)^{j} q^{3 j^{2}-2 j+n} \mathrm{~T}_{1}(n-1,3 j-1 ; q)+\sum_{j=-\infty}^{\infty}(-1)^{j} q^{3 j^{2}+4 j+n+1} \mathrm{~T}_{1}(n-1,3 j+2 ; q) \\
& =0 \text { (by shifting } j \text { to } j-1 \text { in the second term). }
\end{aligned}
$$

Since $P_{0}=\sum_{j=-\infty}^{\infty}(-1)^{j} q^{3 j^{2}+j} \mathrm{U}(0,3 j ; q)=1$, the proof is complete.

The preceeding example was particularly simple. In general, more effort is required in recurrence proofs. Let us now look at a somewhat more intricate example.

Identity 3.17 (Finite form of A.17).

$$
\begin{gathered}
\sum_{j \geqq 0} \sum_{k \geqq 0}(-1)^{k} q^{j^{2}+j+k}\left[\begin{array}{c}
j+k \\
j
\end{array}\right]_{q^{2}}\left[\begin{array}{c}
n-k \\
j
\end{array}\right]_{q^{2}}=\sum_{j=-\infty}^{\infty}(-1)^{j} q^{j(5 j+3) / 2} \mathrm{~T}_{1}\left(n+1,\left\lfloor\frac{5 j+2}{2}\right\rfloor ; q\right) \\
P_{0}=1 \\
P_{1}=q^{2}-q+1 \\
P_{n}=\left(1-q+q^{2 n}\right) P_{n-1}+q P_{n-2} \text { if } n \geqq 2 .
\end{gathered}
$$

Proof. Let

$$
\begin{aligned}
P_{n} & =\sum_{j=-\infty}^{\infty}(-1)^{j} q^{j(5 j+3) / 2} \mathrm{~T}_{1}\left(n+1,\left\lfloor\frac{5 j+2}{2}\right\rfloor ; q\right) \\
& =\sum_{k=-\infty}^{\infty} q^{10 k^{2}+3 k} \mathrm{~T}_{1}(n+1,5 k+1 ; q)-q^{10 k^{2}+13 k+4} \mathrm{~T}_{1}(n+1,5 k+3 ; q) .
\end{aligned}
$$

We need to show that $P_{n}-\left(1-q+q^{n}\right) P_{n-1}-q P_{n-2}=0$ if $n \geqq 2$ and that the appropriate initial conditions hold. 


$$
\begin{aligned}
& P_{n}-P_{n-1}+q P_{n-1}-q^{2 n} P_{n-1}-q P_{n-2} \\
= & \sum_{k=-\infty}^{\infty} q^{10 k^{2}+3 k} \mathrm{~T}_{1}(n+1,5 k+1 ; q)-\sum_{k=-\infty}^{\infty} q^{10 k^{2}+3 k} \mathrm{~T}_{1}(n, 5 k+1 ; q) \\
+ & \sum_{k=-\infty}^{\infty} q^{10 k^{2}+3 k+1} \mathrm{~T}_{1}(n, 5 k+1 ; q)-\sum_{k=-\infty}^{\infty} q^{10 k^{2}+3 k+2 n} \mathrm{~T}_{1}(n, 5 k+1 ; q) \\
& -\sum_{k=-\infty}^{\infty} q^{10 k^{2}+3 k+1} \mathrm{~T}_{1}(n-1,5 k+1 ; q)-\sum_{k=-\infty}^{\infty} q^{10 k^{2}+13 k+4} \mathrm{~T}_{1}(n+1,5 k+3 ; q) \\
+ & \sum_{k=-\infty}^{\infty} q^{10 k^{2}+13 k+4} \mathrm{~T}_{1}(n, 5 k+3 ; q)-\sum_{k=-\infty}^{\infty} q^{10 k^{2}+13 k+5} \mathrm{~T}_{1}(n, 5 k+3 ; q) \\
+ & \sum_{k=-\infty}^{\infty} q^{10 k^{2}+13 k+2 n+4} \mathrm{~T}_{1}(n, 5 k+3 ; q)+\sum_{k=-\infty}^{\infty} q^{10 k^{2}+13 k+5} \mathrm{~T}_{1}(n-1,5 k+3 ; q)
\end{aligned}
$$

Apply (1.25) to the first, third, sixth, and ninth terms to obtain:

$$
\begin{aligned}
= & \sum_{k=-\infty}^{\infty} q^{10 k^{2}+3 k} \mathrm{~T}_{1}(n, 5 k+1 ; q)+\sum_{k=-\infty}^{\infty} q^{10 k^{2}+8 k+n+2} \mathrm{~T}_{0}(n, 5 k+2 ; q) \\
& +\sum_{k=-\infty}^{\infty} q^{10 k^{2}-2 k+n} \mathrm{~T}_{0}(n, 5 k ; q)-\sum_{k=-\infty}^{\infty} q^{10 k^{2}+3 k} \mathrm{~T}_{1}(n, 5 k+1 ; q) \\
& +\sum_{k=-\infty}^{\infty} q^{10 k^{2}+3 k+1} \mathrm{~T}_{1}(n-1,5 k+1 ; q)+\sum_{k=-\infty}^{\infty} q^{10 k^{2}+8 k+n+2} \mathrm{~T}_{0}(n-1,5 k+2 ; q) \\
& +\sum_{k=-\infty}^{\infty} q^{10 k^{2}-2 k+n} \mathrm{~T}_{0}(n-1,5 k ; q)-\sum_{k=-\infty}^{\infty} q^{10 k^{2}+3 k+2 n} \mathrm{~T}_{1}(n, 5 k+1 ; q) \\
& -\sum_{k=-\infty}^{\infty} q^{10 k^{2}+3 k+1} \mathrm{~T}_{1}(n-1,5 k+1 ; q)-\sum_{k=-\infty}^{\infty} q^{10 k^{2}+13 k+4} \mathrm{~T}_{1}(n, 5 k+3 ; q) \\
& -\sum_{k=-\infty}^{\infty} q^{10 k^{2}+18 k+n+8} \mathrm{~T}_{0}(n, 5 k+4 ; q)-\sum_{k=-\infty}^{\infty} q^{10 k^{2}+8 k+n+2} \mathrm{~T}_{0}(n, 5 k+2 ; q) \\
& +\sum_{k=-\infty}^{\infty} q^{10 k^{2}+13 k+4} \mathrm{~T}_{1}(n, 5 k+3 ; q)-\sum_{k=-\infty}^{\infty} q^{10 k^{2}+13 k+5} \mathrm{~T}_{1}(n-1,5 k+3 ; q) \\
& -\sum_{k=-\infty}^{\infty} q^{10 k^{2}+18 k+n+8} \mathrm{~T}_{0}(n-1,5 k+4 ; q)-\sum_{k=-\infty}^{\infty} q^{10 k^{2}+8 k+n+2} \mathrm{~T}_{0}(n-1,5 k+2 ; q) \\
& +\sum_{k=-\infty}^{\infty} q^{10 k^{2}+13 k+2 n+4} \mathrm{~T}_{1}(n, 5 k+3 ; q)+\sum_{k=-\infty}^{\infty} q^{10 k^{2}+13 k+5} \mathrm{~T}_{1}(n-1,5 k+3 ; q)
\end{aligned}
$$


whereupon the first terms cancels the fourth, the second cancels the twelfth, the fifth cancels the ninth, the sixth cancels the sixteenth, the tenth cancels the thirteenth, and the fourteenth cancels the eighteenth leaving

$$
\begin{aligned}
= & \sum_{k=-\infty}^{\infty} q^{10 k^{2}-2 k+n} \mathrm{~T}_{0}(n, 5 k ; q)+\sum_{k=-\infty}^{\infty} q^{10 k^{2}-2 k+n} \mathrm{~T}_{0}(n-1,5 k ; q) \\
& -\sum_{k=-\infty}^{\infty} q^{10 k^{2}+3 k+2 n} \mathrm{~T}_{1}(n, 5 k+1 ; q)-\sum_{k=-\infty}^{\infty} q^{10 k^{2}+18 k+n+8} \mathrm{~T}_{0}(n, 5 k+4 ; q) \\
& -\sum_{k=-\infty}^{\infty} q^{10 k^{2}+18 k+n+8} \mathrm{~T}_{0}(n-1,5 k+4 ; q)+\sum_{k=-\infty}^{\infty} q^{10 k^{2}+13 k+2 n+4} \mathrm{~T}_{1}(n, 5 k+3 ; q)
\end{aligned}
$$

Next, we apply (1.26) to the first and fourth terms, and (1.25) to the third and sixth terms:

$$
\begin{aligned}
= & \sum_{k=-\infty}^{\infty} q^{10 k^{2}-2 k+n} \mathrm{~T}_{0}(n-1,5 k-1 ; q)+\sum_{k=-\infty}^{\infty} q^{10 k^{2}+3 k+2 n} \mathrm{~T}_{1}(n-1,5 k ; q) \\
& +\sum_{k=-\infty}^{\infty} q^{10 k^{2}+8 k+3 n} \mathrm{~T}_{0}(n-1,5 k+1 ; q)+\sum_{k=-\infty}^{\infty} q^{10 k^{2}-2 k+n} \mathrm{~T}_{0}(n-1,5 k ; q) \\
& -\sum_{k=-\infty}^{\infty} q^{10 k^{2}+3 k+2 n} \mathrm{~T}_{1}(n-1,5 k+1 ; q)-\sum_{k=-\infty}^{\infty} q^{10 k^{2}+8 k+3 n+1} \mathrm{~T}_{0}(n-1,5 k+2 ; q) \\
& -\sum_{k=-\infty}^{\infty} q^{10 k^{2}-2 k+3 n-1} \mathrm{~T}_{0}(n-1,5 k ; q)-\sum_{k=-\infty}^{\infty} q^{10 k^{2}-18 k+n+8} \mathrm{~T}_{0}(n-1,5 k-5 ; q) \\
& -\sum_{k=-\infty}^{\infty} q^{10 k^{2}-13 k+2 n+4} \mathrm{~T}_{1}(n-1,5 k-4 ; q)-\sum_{k=-\infty}^{\infty} q^{10 k^{2}-8 k+3 n} \mathrm{~T}_{0}(n-1,5 k-3 ; q) \\
& -\sum_{k=-\infty}^{\infty} q^{10 k^{2}-18 k+n+8} \mathrm{~T}_{0}(n-1,5 k-4 ; q)+\sum_{k=-\infty}^{\infty} q^{10 k^{2}+13 k+2 n+4} \mathrm{~T}_{1}(n-1,5 k+3 ; q) \\
& +\sum_{k=-\infty}^{\infty} q^{10 k^{2}+18 k+3 n+7} \mathrm{~T}_{0}(n-1,5 k+4 ; q)+\sum_{k=-\infty}^{\infty} q^{10 k^{2}+8 k+3 n+1} \mathrm{~T}_{0}(n-1,5 k+2 ; q)
\end{aligned}
$$

In the above, the sixth and fourteenth terms cancel each other. The second, third, fifth, and seventh terms sum to zero by (1.31). Next, replace $k$ by $-k$ and apply (1.39) to the ninth term. Replace $k$ by $-k$ and apply (1.38) to the tenth term. Then, the ninth, tenth, twelfth, and thirteen terms sum to zero by (1.31). Now we have

$$
\begin{aligned}
& \sum_{k=-\infty}^{\infty} q^{10 k^{2}-2 k+n} \mathrm{~T}_{0}(n-1,5 k-1 ; q)+\sum_{k=-\infty}^{\infty} q^{10 k^{2}-2 k+n} \mathrm{~T}_{0}(n-1,5 k ; q) \\
- & \sum_{k=-\infty}^{\infty} q^{10 k^{2}-18 k+n+8} \mathrm{~T}_{0}(n-1,5 k-5 ; q)-\sum_{k=-\infty}^{\infty} q^{10 k^{2}-18 k+n+8} \mathrm{~T}_{0}(n-1,5 k-4 ; q)
\end{aligned}
$$


In the third and fourth terms, replace $k$ by $1-k$ and apply (1.38). Then the first term will cancel the fourth and the second will cancel the third:

$$
=0 \text {. }
$$

Upon verifying the easily checked initial conditions, the proof is complete.

Further examples of recurrence proofs may be found elsewhere in the literature: Santos [60, Chapter 2] contains proofs of Identities 3.29 and 3.38-b. Santos proves Identity 3.20 in [61]. Proofs of Identities 3.8 and 3.12 are given in Santos and Sills [62, Theorems 1 and 3].

As one can imagine, once higher order recurrences are encountered, the process of giving a recurrence proof becomes increasingly tedious. Merely transcribing one line to the next without ever inadvertantly changing a " 0 " to a " 1 " or a "+" to "-" is more than one could reasonbly expect a human to do. Accordingly, I have written the recpf Maple package to assist the human mathematician in successfully carrying out recurrence proofs in an efficient and minimally tedious manner. The recpf package is documented in [67], and Maple worksheets containing recurrence proofs for the identities listed in $\S 3$ are available from my web site: http://www.math.psu.edu/sills (through August 2003), http://www.math.rutgers.edu/ ${ }^{\sim}$ sills (starting September 2003).

\section{$5 \quad$ Rogers-Ramanujan Reciprocal Duality}

\section{$5.1 \quad$ Introduction}

In [7], Andrews demonstrated a type of duality relationship that exists among a few sets of Rogers-Ramanujan type identities. The (reciprocal) dual of a polynomial

$$
a_{n} q^{n}+a_{n-1} q^{n-1}+a_{n-2} q^{n-2}+\cdots+a_{1} q+a_{0}
$$

is

$$
a_{0} q^{n}+a_{1} q^{n-1}+a_{2} q^{n-2}+\cdots+a_{n-1} q+a_{n} .
$$

Equivalently, the reciprocal of $P(q)$ is

$$
q^{\operatorname{deg}(P(q))} P\left(q^{-1}\right) .
$$

If $q^{\operatorname{deg}(P(q))} P\left(q^{-1}\right)=P(q)$, the associated identity is called self-dual. Let us work through an example of calculating the dual of an identity: Consider, say, identity 10 from Slater's list. A finite form (3.10) is

If $n$ is a nonnegative integer, then

$$
\begin{gathered}
\sum_{i \geqq 0} \sum_{j \geqq 0} \sum_{k \geqq 0} q^{j^{2}+i^{2}-i+k}\left[\begin{array}{l}
j \\
i
\end{array}\right]_{q^{2}}\left[\begin{array}{c}
j+k-1 \\
k
\end{array}\right]_{q^{2}}\left[\begin{array}{c}
n-i-k \\
j
\end{array}\right]_{q^{2}} \\
=\sum_{j=-\infty}^{\infty} q^{2 j^{2}+j}\left[\mathrm{~T}_{0}(n, 2 j ; q)+\mathrm{T}_{0}(n-1,2 j ; q) .\right]
\end{gathered}
$$


An examination of the first few cases $n=0,1,2$, etc., convinces one that the degree of the polynomial is $n^{2}$. Thus, by (3.10) and (5.1), the dual polynomials of (3.10) can be represented by either of the two forms

$$
\begin{gathered}
\sum_{i \geqq 0} \sum_{j \geqq 0} \sum_{k \geqq 0} q^{n^{2}-\left(j^{2}+i^{2}-i+k\right)}\left[\begin{array}{l}
j \\
i
\end{array}\right]_{1 / q^{2}}\left[\begin{array}{c}
j+k-1 \\
k
\end{array}\right]_{1 / q^{2}}\left[\begin{array}{c}
n-i-k \\
j
\end{array}\right]_{1 / q^{2}} \\
=\sum_{j=-\infty}^{\infty} q^{n^{2}-\left(2 j^{2}+j\right)}\left[\mathrm{T}_{0}(n, 2 j ; 1 / q)+\mathrm{T}_{0}(n-1,2 j ; 1 / q)\right]
\end{gathered}
$$

Applying (1.42) and (1.41) on the righthand side and (1.7) on the left hand side, we obtain

$$
\begin{gathered}
\sum_{h \geqq 0} \sum_{i \geqq 0} \sum_{k \geqq 0} q^{k+i+2 i(h+i+k)+(h+k)^{2}}\left[\begin{array}{c}
n-i-h-k \\
i
\end{array}\right]_{q^{2}}\left[\begin{array}{c}
n-i-h-1 \\
k
\end{array}\right]_{q^{2}}\left[\begin{array}{c}
n-i-k \\
h
\end{array}\right]_{q^{2}} \\
=\sum_{j=-\infty}^{\infty} q^{2 j^{2}-j}\left(\begin{array}{c}
n, 2 j ; q^{2} \\
2 j
\end{array}\right)_{2}+q^{2 j^{2}-j+2 n-1}\left(\begin{array}{c}
n-1,2 j ; q^{2} \\
2 j
\end{array}\right)_{2}
\end{gathered}
$$

We can suppose that $|q|<1$ and let $n \rightarrow \infty$ in (5.10f) to obtain a Rogers-Ramanujan type identity. First, we consider the lefthand side:

$$
\begin{aligned}
& \lim _{n \rightarrow \infty} \sum_{h, i, k \geqq 0} q^{k+i+2 i(h+i+k)+(h+k)^{2}}\left[\begin{array}{c}
n-i-h-k \\
i
\end{array}\right]_{q^{2}}\left[\begin{array}{c}
n-i-h-1 \\
k
\end{array}\right]_{q^{2}}\left[\begin{array}{c}
n-i-k \\
h
\end{array}\right]_{q^{2}} \\
= & \lim _{n \rightarrow \infty} \sum_{h, i, k \geqq 0} \frac{q^{k+i+2 i(h+i+k)+(h+k)^{2}}\left(q^{2} ; q^{2}\right)_{n-i-h-k}\left(q^{2} ; q^{2}\right)_{n-i-h-1}\left(q^{2} ; q^{2}\right)_{n-i-k}}{\left(q^{2} ; q^{2}\right)_{i}\left(q^{2} ; q^{2}\right)_{n-2 i-j-k}\left(q^{2} ; q^{2}\right)_{k}\left(q^{2} ; q^{2}\right)_{n-i-k-h-1}\left(q^{2} ; q^{2}\right)_{h}\left(q^{2} ; q^{2}\right)_{n-i-k-h}} \\
= & \sum_{h=0}^{\infty} \sum_{i=0}^{\infty} \sum_{k=0}^{\infty} \frac{q^{k+i+2 i(h+i+k)+(h+k)^{2}}}{\left(q^{2} ; q^{2}\right)_{h}\left(q^{2} ; q^{2}\right)_{i}\left(q^{2} ; q^{2}\right)_{k}}
\end{aligned}
$$

Next, we consider the righthand side:

$$
\begin{aligned}
& \lim _{n \rightarrow \infty} \sum_{j=-\infty}^{\infty} q^{2 j^{2}-j}\left(\begin{array}{c}
n, 2 j ; q^{2} \\
2 j
\end{array}\right)_{2}+q^{2 j^{2}-j+2 n-1}\left(\begin{array}{c}
n-1,2 j ; q^{2} \\
2 j
\end{array}\right)_{2} \\
= & \left.\frac{1}{\left(q^{2} ; q^{2}\right)_{\infty}}\left[\sum_{j=-\infty}^{\infty} q^{2 j^{2}-j}+0\right] \quad \text { (by }(1.46) \text { and since }|q|<1\right) \\
= & \frac{1}{\left(q^{2} ; q^{2}\right)_{\infty}}\left(-q,-q^{3}, q^{4} ; q^{4}\right)_{\infty} \quad \text { (by Theorem 1.55) } \\
= & (-q ; q)_{\infty} \\
= & \prod_{j=1}^{\infty}\left(1+q^{j}\right)
\end{aligned}
$$


Thus, for $|q|<1$,

$$
\sum_{h=0}^{\infty} \sum_{i=0}^{\infty} \sum_{k=0}^{\infty} \frac{q^{k+i+2 i(h+i+k)+(h+k)^{2}}}{\left(q^{2} ; q^{2}\right)_{h}\left(q^{2} ; q^{2}\right)_{i}\left(q^{2} ; q^{2}\right)_{k}}=\prod_{j=1}^{\infty}\left(1+q^{j}\right) .
$$

Note that the triple sum in the preceeding equation can be simplified:

$$
\begin{aligned}
& \sum_{h=0}^{\infty} \sum_{i=0}^{\infty} \sum_{k=0}^{\infty} \frac{q^{k+i+2 i(h+i+k)+(h+k)^{2}}}{\left(q^{2} ; q^{2}\right)_{h}\left(q^{2} ; q^{2}\right)_{i}\left(q^{2} ; q^{2}\right)_{k}} \\
& =\sum_{i=0}^{\infty} \sum_{k=0}^{\infty} \frac{q^{k+i+i^{2}+(i+k)^{2}}}{\left(q^{2} ; q^{2}\right)_{i}\left(q^{2} ; q^{2}\right)_{k}} \sum_{h=0}^{\infty} \frac{q^{h^{2}+(2 i+2 k) h}}{\left(q^{2} ; q^{2}\right)_{h}} \\
& =\sum_{i=0}^{\infty} \sum_{k=0}^{\infty} \frac{q^{k+i+i^{2}+(i+k)^{2}}}{\left(q^{2} ; q^{2}\right)_{i}\left(q^{2} ; q^{2}\right)_{k}}\left(-q^{2 i+2 k+1} ; q^{2}\right)_{\infty} \quad(\text { by }(1.12)) \\
& =\left(-q ; q^{2}\right)_{\infty} \sum_{i=0}^{\infty} \sum_{k=0}^{\infty} \frac{q^{k^{2}+2 i k+2 i^{2}+k+i}}{\left(q^{2} ; q^{2}\right)_{i}\left(q^{2} ; q^{2}\right)_{k}\left(-q ; q^{2}\right)_{i+k}} \\
& \left.=\left(-q ; q^{2}\right)_{\infty} \sum_{i=0}^{\infty} \sum_{K=i}^{\infty} \frac{q^{K^{2}+K+i^{2}}}{\left(q^{2} ; q^{2}\right)_{i}\left(q^{2} ; q^{2}\right)_{K-i}\left(-q ; q^{2}\right)_{K}} \quad \text { (by taking } K=k+i\right) \\
& =\left(-q ; q^{2}\right)_{\infty} \sum_{K=0}^{\infty} \frac{q^{K^{2}+K}}{\left(-q ; q^{2}\right)_{K}} \sum_{i=0}^{K} \frac{q^{i^{2}}}{\left(q^{2} ; q^{2}\right)_{i}\left(q^{2} ; q^{2}\right)_{K-i}} \\
& =\left(-q ; q^{2}\right)_{\infty} \sum_{K=0}^{\infty} \frac{q^{K^{2}+K}}{\left(-q ; q^{2}\right)_{K}\left(q^{2} ; q^{2}\right)_{K}} \sum_{i=0}^{K} \frac{q^{i^{2}}\left(q^{2} ; q^{2}\right)_{K}}{\left(q^{2} ; q^{2}\right)_{i}\left(q^{2} ; q^{2}\right)_{K-i}} \\
& =\left(-q ; q^{2}\right)_{\infty} \sum_{K=0}^{\infty} \frac{q^{K^{2}+K}}{\left(-q ; q^{2}\right)_{K}\left(q^{2} ; q^{2}\right)_{K}} \sum_{i=0}^{K} q^{i^{2}}\left[\begin{array}{c}
K \\
i
\end{array}\right]_{q^{2}} \\
& =\left(-q ; q^{2}\right)_{\infty} \sum_{K=0}^{\infty} \frac{q^{K^{2}+K}\left(-q ; q^{2}\right)_{K}}{\left(-q ; q^{2}\right)_{K}\left(q^{2} ; q^{2}\right)_{K}} \quad(\text { by }(1.10)) \\
& =\left(-q ; q^{2}\right)_{\infty} \sum_{K=0}^{\infty} \frac{q^{K^{2}+K}}{\left(q^{2} ; q^{2}\right)_{K}} .
\end{aligned}
$$

Thus, (5.4) is equivalent to

$$
\left(-q ; q^{2}\right)_{\infty} \sum_{j=0}^{\infty} \frac{q^{j(j+1)}}{\left(q^{2} ; q^{2}\right)_{j}}=\prod_{j=1}^{\infty}\left(1+q^{j}\right)
$$

or, after dividing through by $\left(-q ; q^{2}\right)_{\infty}$,

$$
\sum_{j=0}^{\infty} \frac{q^{j(j+1)}}{\left(q^{2} ; q^{2}\right)_{j}}=\prod_{j=1}^{\infty}\left(1+q^{2 j}\right)
$$


which is Identity A.7. I will not go so far as to say that "Identities A.7 and A.10 are dual" since it was necessary to take the limit as $n \rightarrow \infty$ and to divide out an infinite product in order to obtain Identity A.7 from the dual of (3.10). In fact, it is not hard to show that Identity A.7 is self-dual. Nonetheless, it is clear that (A.7) and (A.10) are very closely related identities.

In some cases, the sequence of polynomials $\left\{P_{n}(q)\right\}_{n=0}^{\infty}$ does not converge, but the subsequence $\left\{P_{2 m}(q)\right\}_{m=0}^{\infty}$ converges to one series, and the subsequence $\left\{P_{2 m+1}(q)\right\}_{m=0}^{\infty}$ converges to a different series. One immediate clue that this may be occuring is when the formula for the degree of the polynomial varies with the parity of $n$. For example, with the finite First Rogers-Ramanujan Identity (3.18), the degree of the polynomial is $n^{2} / 4$ if $n$ is even, and $\left(n^{2}-1\right) / 4$ if $n$ is odd. In cases such as this, we consider the dual of (3.18) to be a pair of identities. The appropriate calculation shows that the dual of "18 even" is identity 79, and the dual of "18 odd" is identity 99.

In $\S 3$, I presented a finitization of each of the Rogers-Ramanujan type identities on Slater's list. In this section, I present the dual polynomial identities for some of the identities appearing in $\S 3$, as well as their limiting cases.

\subsection{Identities}

Observation 5.2. Identity (2) is self-dual.

Observation 5.3. Identity (3) is self dual.

Identity 5.4f (Dual of 3.4).

$$
\begin{gathered}
\sum_{h \geqq 0} \sum_{i \geqq 0} \sum_{k \geqq 0}(-1)^{n-i-h} q^{2 i(i+h+k)+(h+k)^{2}}\left[\begin{array}{c}
n-i-h-k \\
i
\end{array}\right]_{q^{2}}\left[\begin{array}{c}
n-i-h-1 \\
k
\end{array}\right]_{q^{2}} \\
\times\left[\begin{array}{c}
n-i-k \\
h
\end{array}\right]_{q^{2}} \\
=\sum_{j=-\infty}^{\infty}(-1)^{j} \mathrm{t}_{0}(n, j ; q)+\sum_{j=-\infty}^{\infty}(-1)^{j} q^{2 n-1} \mathrm{t}_{0}(n-1, j ; q)
\end{gathered}
$$

Observation 5.5. Identity (5) (with $q$ replaced by $-q$ ) is self dual.

Identity 5.6f-even (Dual of 3.6 even).

$$
\begin{aligned}
& \sum_{i \geqq 0} \sum_{j \geqq 0} \sum_{k \geqq 0} q^{j^{2}+i(i+1) / 2+k}\left[\begin{array}{c}
m-j \\
i
\end{array}\right]_{q}\left[\begin{array}{c}
m-j+k-1 \\
k
\end{array}\right]_{q^{2}}\left[\begin{array}{c}
j+m-i-2 k \\
m-j
\end{array}\right]_{q} \\
& \quad=\sum_{j=-\infty}^{\infty} q^{j(3 j-1)}\left[\begin{array}{c}
2 m \\
m+3 j
\end{array}\right]_{q}+q^{j(3 j+4)+m+1}\left[\begin{array}{c}
2 m-1 \\
m+3 j+1
\end{array}\right]_{q}
\end{aligned}
$$

Identity 5.6-even (Dual of A.6 even).

$$
\sum_{i=0}^{\infty} \sum_{j=0}^{\infty} \sum_{k=0}^{\infty} \frac{q^{j^{2}+i(i+1) / 2+k}}{(q ; q)_{i}\left(q^{2} ; q^{2}\right)_{k}(q ; q)_{2 j-i-2 k}}=\prod_{n=1}^{\infty} \frac{\left(1+q^{6 n-2}\right)\left(1+q^{6 n-4}\right)\left(1-q^{6 n}\right)}{1-q^{n}}
$$


Identity 5.6f-odd (Dual of 3.6 odd).

$$
\begin{gathered}
\sum_{i \geqq 0} \sum_{j \geqq 0} \sum_{k \geqq 0} q^{j^{2}+j+i(i+1) / 2+k}\left[\begin{array}{c}
m-j \\
i
\end{array}\right]_{q}\left[\begin{array}{c}
m-j+k-1 \\
k
\end{array}\right]_{q^{2}}\left[\begin{array}{c}
j+m-i-2 k+1 \\
m-j
\end{array}\right]_{q} \\
=\sum_{j=-\infty}^{\infty} q^{j(3 j-1)+n}\left[\begin{array}{c}
2 m \\
m+3 j
\end{array}\right]_{q}+q^{j(3 j+4) 1}\left[\begin{array}{c}
2 m+1 \\
m+3 j+2
\end{array}\right]_{q}
\end{gathered}
$$

\section{Identity 5.6-odd (Dual of A.6 odd).}

$$
\sum_{i=0}^{\infty} \sum_{j=0}^{\infty} \sum_{k=0}^{\infty} \frac{q^{j^{2}+j+i(i+1) / 2+k}}{(q ; q)_{i}\left(q^{2} ; q^{2}\right)_{k}(q ; q)_{2 j-i-2 k+1}}=\prod_{n=1}^{\infty} \frac{\left(1+q^{6 n-1}\right)\left(1+q^{6 n-5}\right)\left(1-q^{6 n}\right)}{1-q^{n}}
$$

Observation 5.8. The dual of (8) is (12).

Identity 5.10f (Dual of 3.10).

$$
\begin{gathered}
\sum_{h \geqq 0} \sum_{i \geqq 0} \sum_{k \geqq 0} q^{k+i+2 i(h+i+k)+(h+k)^{2}}\left[\begin{array}{c}
n-i-h-k \\
i
\end{array}\right]_{q^{2}}\left[\begin{array}{c}
n-i-h-1 \\
k
\end{array}\right]_{q^{2}}\left[\begin{array}{c}
n-i-k \\
h
\end{array}\right]_{q^{2}} \\
=\sum_{j=-\infty}^{\infty} q^{2 j^{2}-j}\left(\begin{array}{c}
n, 2 j ; q^{2} \\
2 j
\end{array}\right)_{2}+q^{2 j^{2}-j+2 n-1}\left(\begin{array}{c}
n-1,2 j ; q^{2} \\
2 j
\end{array}\right)_{2}
\end{gathered}
$$

Identity 5.10 (Dual of A.10).

$$
\begin{gathered}
\sum_{h=0}^{\infty} \sum_{i=0}^{\infty} \sum_{k=0}^{\infty} \frac{q^{k+i+2 i(h+i+k)+(h+k)^{2}}}{\left(q^{2} ; q^{2}\right)_{h}\left(q^{2} ; q^{2}\right)_{i}\left(q^{2} ; q^{2}\right)_{k}}=\prod_{j=1}^{\infty}\left(1+q^{j}\right) \\
\prod_{j=0}^{\infty}\left(1+q^{2 j-1}\right) \sum_{j=0}^{\infty} \frac{q^{j(j+1)}}{\left(q^{2} ; q^{2}\right)_{j}}=\prod_{j=1}^{\infty}\left(1+q^{j}\right)
\end{gathered}
$$

Identity 5.11f (Dual of 3.11).

$$
\begin{gathered}
\sum_{h \geqq 0} \sum_{i \geqq 0} q^{(h+i)^{2}+i(i+1)}\left[\begin{array}{c}
n-i-h \\
i
\end{array}\right]_{q^{2}}\left[\begin{array}{c}
2 n-2 i-h+1 \\
h
\end{array}\right]_{q^{2}} \\
=\sum_{j=-\infty}^{\infty}(-1)^{j} q^{3 j^{2}+j}\left[\begin{array}{c}
2 n+1 \\
n+3 j+1
\end{array}\right]_{q}
\end{gathered}
$$

Identity 5.11 (Dual of A.11).

$$
\sum_{h=0}^{\infty} \sum_{i=0}^{\infty} \frac{q^{(h+i)^{2}+i(i+1)}}{\left(q^{2} ; q^{2}\right)_{i}(q ; q)_{h}}=\prod_{j=1}^{\infty}\left(1+q^{j}\right)
$$

Observation 5.12. The dual of (12) is (8). 
Observation 5.14-even. The dual of (14 even) is (99).

Observation 5.14-odd. The dual of (14 odd) is (96).

Identity 5.16f (Dual of 3.16).

$$
\begin{gathered}
\sum_{h \geqq 0} \sum_{k \geqq 0}(-1)^{k} q^{2(h+k)+(h+k)^{2}}\left[\begin{array}{c}
n-h-1 \\
k
\end{array}\right]_{q^{2}}\left[\begin{array}{c}
n-k \\
h
\end{array}\right]_{q^{2}} \\
=\sum_{k=-\infty}^{\infty} q^{15 k^{2}+7 k}\left(\begin{array}{c}
n+1,5 k+1 ; q^{2} \\
5 k+1
\end{array}\right)_{2}-q^{15 k^{2}+17 k+4}\left(\begin{array}{c}
n+1,5 k+3 ; q^{2} \\
5 k+3
\end{array}\right)_{2} \\
+q^{15 k^{2}+7 k+1+2 n}\left(\begin{array}{c}
n+1,5 k+1 ; q^{2} \\
5 k+1
\end{array}\right)_{2}-q^{15 k^{2}+17 k+5+2 n}\left(\begin{array}{c}
n+1,5 k+3 ; q^{2} \\
5 k+3
\end{array}\right)_{2}
\end{gathered}
$$

Identity 5.16 (Dual of A.16). Note: The dual of (A.16) with q replaced by $-q$ is equivalent to $\left(q ; q^{2}\right)_{\infty} \times(A .96)$.

$$
\begin{gathered}
\sum_{h=0}^{\infty} \sum_{k=0}^{\infty} \frac{(-1)^{k} q^{2(h+k)+(h+k)^{2}}}{\left(q^{2} ; q^{2}\right)_{\infty}\left(q^{2} ; q^{2}\right)_{h}} \\
=\prod_{j=1}^{\infty} \frac{\left(1-q^{10 j-4}\right)\left(1-q^{10 j-6}\right)\left(1-q^{10 j}\right)\left(1-q^{20 j-2}\right)\left(1-q^{20 j-18}\right)}{\left(1-q^{2 j}\right)}
\end{gathered}
$$

Identity 5.17f (Dual of 3.17).

$$
\begin{gathered}
\sum_{h \geqq 0} \sum_{k \geqq 0}(-1)^{k} q^{(h+k)^{2}+h}\left[\begin{array}{c}
n-h \\
k
\end{array}\right]_{q^{2}}\left[\begin{array}{c}
n-k \\
h
\end{array}\right]_{q^{2}} \\
=\sum_{k=-\infty}^{\infty} q^{15 k^{2}+2 k}\left(\begin{array}{c}
n+1,5 k ; q \\
5 k+1
\end{array}\right)_{2}-q^{15 k^{2}+12 k+2}\left(\begin{array}{c}
n+1,5 k+2 ; q \\
5 k+3
\end{array}\right)_{2}
\end{gathered}
$$

Identity 5.17 (Dual of A.17). Note: The dual of (A.17) with q replaced by $-q$ is equivalent to $\left(-q^{2} ; q^{2}\right)_{\infty} \times($ A.20) .

$$
\begin{gathered}
\sum_{h=0}^{\infty} \sum_{k=0}^{\infty} \frac{(-1)^{k} q^{(h+k)^{2}+h}}{\left(q^{2} ; q^{2}\right)_{k}\left(q^{2} ; q^{2}\right)_{h}} \\
=\prod_{j=1}^{\infty} \frac{\left(1-q^{10 j-1}\right)\left(1-q^{10 j-9}\right)\left(1-q^{10 j}\right)\left(1-q^{20 j-8}\right)\left(1-q^{20 j-12}\right)}{\left(1-q^{2 j}\right)}
\end{gathered}
$$

Observation 5.18-even. The dual of (18 even) is (79).

Observation 5.18-odd. The dual of (18 odd) is (94). 


\section{Identity 5.20f (Dual of 3.20).}

$$
\begin{gathered}
\sum_{h \geqq 0 \geqq 0} \sum_{k \geqq 0} q^{(h+k)^{2}}\left[\begin{array}{c}
n-h-1 \\
k
\end{array}\right]_{q^{2}}\left[\begin{array}{c}
n-k \\
h
\end{array}\right]_{q} \\
=\sum_{j=-\infty}^{\infty}(-1)^{j} q^{j(15 j-1)}\left(\begin{array}{c}
n, 5 j ; q^{2} \\
5 j
\end{array}\right)_{2}+(-1)^{j} q^{j(15 j-1)+2 n-1}\left(\begin{array}{c}
n-1,5 j+1 ; q^{2} \\
5 j+1
\end{array}\right)_{2} \\
-(-1)^{j} q^{j(15 j+19)+6}\left(\begin{array}{c}
n, 5 j+3 ; q^{2} \\
5 j+3
\end{array}\right)_{2}-(-1)^{j} q^{j(15 j+19)+2 n+5}\left(\begin{array}{c}
n-1,5 j+3 ; q^{2} \\
5 j+3
\end{array}\right)_{2}
\end{gathered}
$$

Identity 5.20 (Dual of A.20). Note: The dual of (A.20) with $q$ replaced by $-q$ is equivalent to $\left(q ; q^{2}\right)_{\infty} \times(A .79)$.

$$
\begin{gathered}
\sum_{h=0}^{\infty} \sum_{k=0}^{\infty} \frac{q^{(h+k)^{2}}}{\left(q^{2} ; q^{2}\right)_{h}\left(q^{2} ; q^{2}\right)_{k}} \\
=\prod_{j=1}^{\infty} \frac{\left(1-q^{10 j-2}\right)\left(1-q^{10 j-8}\right)\left(1-q^{10 j}\right)\left(1-q^{20 j-14}\right)\left(1-q^{20 j-6}\right)}{\left(1-q^{2 j}\right)}
\end{gathered}
$$

Identity 5.25f (Dual of 3.25).

$$
\begin{aligned}
& \sum_{h \geqq 0} \sum_{i \geqq 0} \sum_{k \geqq 0}(-1)^{k} q^{2 i(i+h+k)+(h+k)^{2}}\left[\begin{array}{c}
n-i-h-k \\
i
\end{array}\right]_{q^{2}}\left[\begin{array}{c}
n-i-h \\
k
\end{array}\right]_{q^{2}}\left[\begin{array}{c}
n-i-k \\
h
\end{array}\right]_{q^{2}} \\
& =\sum_{j=-\infty}^{\infty}(-1)^{j} q^{6 j^{2}}\left(\begin{array}{c}
n, 3 j ; q^{2} \\
3 j
\end{array}\right)_{2}+\sum_{j=-\infty}^{\infty}(-1)^{j} q^{6 j^{2}+6 j+1}\left(\begin{array}{c}
n, 3 j+1 ; q^{2} \\
3 j+1
\end{array}\right)_{2}
\end{aligned}
$$

Identity 5.25 (Dual of A.25).

$$
\begin{gathered}
\sum_{h \geqq 0} \sum_{i \geqq 0} \sum_{k \geqq 0} \frac{(-1)^{k} q^{2 i(h+i+k)+(h+k)^{2}}}{\left(q^{2} ; q^{2}\right)_{h}\left(q^{2} ; q^{2}\right)_{i}\left(q^{2} ; q^{2}\right)_{k}}=\prod_{j=1}^{\infty} \frac{\left(1-q^{12 j-6}\right)^{2}\left(1-q^{12 j}\right)}{\left(1-q^{2 j}\right)} \\
\prod_{j=1}^{\infty}\left(1+q^{2 j-1}\right) \sum_{h=0}^{\infty} \sum_{i=0}^{\infty} \frac{(-1)^{k} q^{(h+i)^{2}+i^{2}}}{\left(q^{2} ; q^{2}\right)_{i}\left(q^{2} ; q^{2}\right)_{h}\left(-q ; q^{2}\right)_{h+i}}=\prod_{j=1}^{\infty} \frac{\left(1-q^{12 j-6}\right)^{2}\left(1-q^{12 j}\right)}{\left(1-q^{2 j}\right)}
\end{gathered}
$$

Identity 5.27f-even (Dual of 3.27 even).

$$
\begin{gathered}
\sum_{i \geqq 0} \sum_{J \geqq 0} \sum_{k \geqq 0} \sum_{l \geqq 0}(-1)^{l} q^{2 J^{2}+2 J+i^{2}-k}\left[\begin{array}{c}
n-J \\
i
\end{array}\right]_{q^{2}}\left[\begin{array}{c}
n-J+k \\
k
\end{array}\right]_{q^{2}}\left[\begin{array}{c}
n-J+l-1 \\
l
\end{array}\right]_{q^{2}} \\
\times\left[\begin{array}{c}
n+J-i-k-l \\
2 j-i-k-l
\end{array}\right]_{q^{2}} \\
=\sum_{j=-\infty}^{\infty} q^{2 j(3 j+1)}\left[\begin{array}{c}
2 m+1 \\
m+3 j+1
\end{array}\right]_{q^{2}}-q^{2 j(3 j+2)+2 m+1}\left[\begin{array}{c}
2 m \\
m+3 j+1
\end{array}\right]_{q^{2}}
\end{gathered}
$$


Identity 5.27-even (Dual of A.27 even).

$$
\begin{gathered}
\sum_{i=0}^{\infty} \sum_{J=0}^{\infty} \sum_{k=0}^{\infty} \sum_{l=0}^{\infty} \frac{(-1)^{l} q^{2 J^{2}+2 J+i^{2}-k}}{\left(q^{2} ; q^{2}\right)_{i}\left(q^{2} ; q^{2}\right)_{k}\left(q^{2} ; q^{2}\right)_{l}\left(q^{2} ; q^{2}\right)_{2 j-i-k-l}} \\
=\prod_{j=1}^{\infty} \frac{\left(1+q^{12 j-4}\right)\left(1+q^{12 j-8}\right)\left(1-q^{12 j}\right)}{\left(1-q^{2 j}\right)}
\end{gathered}
$$

Identity 5.28f (Dual of 3.28).

$$
\begin{gathered}
\sum_{h \geqq 0} \sum_{i \geqq 0} q^{(h+i)^{2}+i^{2}}\left[\begin{array}{c}
n-i-h \\
i
\end{array}\right]_{q^{2}}\left[\begin{array}{c}
2 n-2 i-h+1 \\
h
\end{array}\right]_{q} \\
=\sum_{j=-\infty}^{\infty}(-1)^{j} q^{j(6 j+1)}\left(\begin{array}{c}
n+1,3 j ; q^{2} \\
3 j+1
\end{array}\right)_{2}
\end{gathered}
$$

Identity 5.28 (Dual of A.28).

$$
\begin{gathered}
\sum_{h=0}^{\infty} \sum_{i=0}^{\infty} \frac{q^{i^{2}+(h+i)^{2}}}{\left(q^{2} ; q^{2}\right)_{i}(q ; q)_{h}} \\
=\prod_{j=1}^{\infty} \frac{\left(1+q^{3 j-1}\right)\left(1+q^{3 j-2}\right)\left(1-q^{3 j}\right)}{\left(1-q^{2 j}\right)}
\end{gathered}
$$

Identity 5.29f (Dual of 3.29).

$$
\begin{aligned}
& \sum_{h \geqq 0} \sum_{i \geqq 0} q^{(h+i)^{2}+i^{2}}\left[\begin{array}{c}
n-h-i \\
i
\end{array}\right]_{q^{2}}\left[\begin{array}{c}
2 n-2 i-h \\
h
\end{array}\right]_{q} \\
= & \sum_{k=-\infty}^{\infty} q^{6 k^{2}-k}\left(\begin{array}{c}
n, 3 k ; q^{2} \\
3 k
\end{array}\right)_{2}+q^{6 k^{2}+5 k+1}\left(\begin{array}{c}
n, 3 k+1 ; q^{2} \\
3 k+1
\end{array}\right)_{2}
\end{aligned}
$$

Identity 5.29 (Dual of A.29).

$$
\sum_{h=0}^{\infty} \sum_{i=0}^{\infty} \frac{q^{i^{2}+(i+h)^{2}}}{\left(q^{2} ; q^{2}\right)_{i}(q ; q)_{h}}=\prod_{j=1}^{\infty} \frac{\left(1+q^{3 j-1}\right)\left(1+q^{3 j-2}\right)\left(1-q^{3 j}\right)}{\left(1-q^{2 j}\right)}
$$

\section{Identity 5.31f-even (Dual of 3.31 even).}

$$
\begin{gathered}
\sum_{J, k, L \geqq 0}(-1)^{k+L} q^{2 J^{2}+2 J-k}\left[\begin{array}{c}
m-J+k \\
k
\end{array}\right]_{q^{2}}\left[\begin{array}{c}
m-J+L-1 \\
L
\end{array}\right]_{q^{2}}\left[\begin{array}{c}
m+J-k-L \\
m-J
\end{array}\right]_{q^{2}} \\
\quad=\sum_{k=-\infty}^{\infty} q^{42 k^{2}+4 k}\left[\begin{array}{c}
2 m+1 \\
m+7 k+1
\end{array}\right]_{q^{2}}-q^{42 k^{2}+32 k+6}\left[\begin{array}{c}
2 m+1 \\
m+7 k+3
\end{array}\right]_{q^{2}} \\
+q^{42 k^{2}+60 k+21}\left(\left[\begin{array}{c}
2 m+1 \\
m+7 k+5
\end{array}\right]_{q^{2}}-\left[\begin{array}{c}
2 m+1 \\
m+7 k+6
\end{array}\right]_{q^{2}}\right)
\end{gathered}
$$




\section{Identity 5.31-even (Dual of A.31 even).}

$$
\begin{gathered}
\sum_{J=0}^{\infty} \sum_{k=0}^{\infty} \sum_{L=0}^{\infty} \frac{(-1)^{k+L} q^{2 J^{2}+2 J-k}}{\left(q^{2} ; q^{2}\right)_{k}\left(q^{2} ; q^{2}\right)_{L}\left(q^{2} ; q^{2}\right)_{2 J-k-L}} \\
=\prod_{j=1}^{\infty} \frac{\left(1-q^{28 j-6}\right)\left(1-q^{28 j-22}\right)\left(1-q^{28 j}\right)\left(1-q^{56 j-16}\right)\left(1-q^{56 j-40}\right)}{\left(1-q^{2 j}\right)}
\end{gathered}
$$

\section{Identity 5.31f-odd (Dual of 3.31 odd).}

$$
\begin{gathered}
\sum_{J, k, L \geqq 0}(-1)^{k+L} q^{2 J^{2}+4 J-k+1}\left[\begin{array}{c}
m-J+k \\
k
\end{array}\right]_{q^{2}}\left[\begin{array}{c}
m-J+L-1 \\
L
\end{array}\right]_{q^{2}}\left[\begin{array}{c}
m+J-k-L+1 \\
m-J
\end{array}\right]_{q^{2}} \\
=\sum_{k=-\infty}^{\infty}-q^{42 k^{2}-10 k}\left[\begin{array}{c}
2 m+2 \\
m+7 k
\end{array}\right]_{q^{2}}+q^{42 k^{2}-38 k+8}\left[\begin{array}{c}
2 m+2 \\
m+7 k-2
\end{array}\right]_{q^{2}} \\
+q^{42 k^{2}+18 k+1}\left(\left[\begin{array}{c}
2 m+2 \\
m+7 k+2
\end{array}\right]_{q^{2}}-\left[\begin{array}{c}
2 m+2 \\
m+7 k+3
\end{array}\right]_{q^{2}}\right)
\end{gathered}
$$

Identity 5.31-odd (Dual of A.31 odd).

$$
\begin{gathered}
\sum_{J=0}^{\infty} \sum_{k=0}^{\infty} \sum_{L=0}^{\infty} \frac{(-1)^{k+L} q^{2 J^{2}+4 J-k+1}}{\left(q^{2} ; q^{2}\right)_{k}\left(q^{2} ; q^{2}\right)_{L}\left(q^{2} ; q^{2}\right)_{2 J-k-L}} \\
=\prod_{j=1}^{\infty} \frac{\left(1-q^{28 j-8}\right)\left(1-q^{28 j-20}\right)\left(1-q^{28 j}\right)\left(1-q^{56 j-28}\right)\left(1-q^{56 j-44}\right)}{\left(1-q^{2 j}\right)}
\end{gathered}
$$

\section{Identity 5.32f-even (Dual of 3.32 even).}

$$
\begin{gathered}
\sum_{J, k, L \geqq 0}(-1)^{k+L} q^{2 J^{2}+2 J+k}\left[\begin{array}{c}
m-J+k-1 \\
k
\end{array}\right]_{q^{2}}\left[\begin{array}{c}
m-J+L-1 \\
L
\end{array}\right]_{q^{2}}\left[\begin{array}{c}
m+J-k-L \\
m-J
\end{array}\right]_{q^{2}} \\
=\sum_{k=-\infty}^{\infty} q^{42 k^{2}+8 k}\left[\begin{array}{c}
2 m+1 \\
m+7 k+1
\end{array}\right]_{q^{2}}-q^{42 k^{2}-20 k+2}\left[\begin{array}{c}
2 m+1 \\
m+7 k+6
\end{array}\right]_{q^{2}} \\
+q^{42 k^{2}+22 k+2 n+3}\left[\begin{array}{c}
2 m \\
m+7 k+2
\end{array}\right]_{q^{2}}-q^{42 k^{2}+50 k+2 n+15}\left[\begin{array}{c}
2 m \\
m+7 k+4
\end{array}\right]_{q^{2}}
\end{gathered}
$$

Identity 5.32-even (Dual of A.32 even).

$$
\begin{gathered}
\sum_{J=0}^{\infty} \sum_{k=0}^{\infty} \sum_{L=0}^{\infty} \frac{(-1)^{k+L} q^{2 J^{2}+2 J+k}}{\left(q^{2} ; q^{2}\right)_{k}\left(q^{2} ; q^{2}\right)_{L}\left(q^{2} ; q^{2}\right)_{2 J-k-L}} \\
=\prod_{j=1}^{\infty} \frac{\left(1-q^{28 j-2}\right)\left(1-q^{28 j-26}\right)\left(1-q^{28 j}\right)\left(1-q^{56 j-28}\right)\left(1-q^{56 j-32}\right)}{\left(1-q^{2 j}\right)}
\end{gathered}
$$


Identity 5.32f-odd (Dual of 3.32 odd).

$$
\begin{gathered}
\sum_{J, k, L \geqq 0}(-1)^{k+L} q^{2 J^{2}+4 J+k-1}\left[\begin{array}{c}
m-J+k-1 \\
k
\end{array}\right]_{q^{2}}\left[\begin{array}{c}
m-J+L-1 \\
L
\end{array}\right]_{q^{2}} \\
\times\left[\begin{array}{c}
m+J-k-L+1 \\
m-J
\end{array}\right]_{q^{2}} \\
=\sum_{k=-\infty}^{\infty}-q^{42 k^{2}-22 k}\left[\begin{array}{c}
2 m+2 \\
m+7 k+3
\end{array}\right]_{q^{2}}+q^{42 k^{2}-34 k+4}\left[\begin{array}{c}
2 m+2 \\
m+7 k-2
\end{array}\right]_{q^{2}} \\
+q^{42 k^{2}+8 k 2 m-1}\left[\begin{array}{c}
2 m+1 \\
m+7 k+1
\end{array}\right]_{q^{2}}-q^{42 k^{2}+64 k+2 m+23}\left[\begin{array}{c}
2 m+1 \\
m+7 k+6
\end{array}\right]_{q^{2}}
\end{gathered}
$$

Identity 5.32-odd (Dual of A.32 odd).

$$
\begin{gathered}
\sum_{J=0}^{\infty} \sum_{k=0}^{\infty} \sum_{L=0}^{\infty} \frac{(-1)^{k+L} q^{2 J^{2}+4 J+k-1}}{\left(q^{2} ; q^{2}\right)_{k}\left(q^{2} ; q^{2}\right)_{L}\left(q^{2} ; q^{2}\right)_{2 J-k-L+1}} \\
=\prod_{j=1}^{\infty} \frac{\left(1-q^{28 j-4}\right)\left(1-q^{28 j-24}\right)\left(1-q^{28 j}\right)\left(1-q^{56 j-20}\right)\left(1-q^{56 j-36}\right)}{\left(1-q^{2 j}\right)}
\end{gathered}
$$

\section{Identity 5.33f-even (Dual of 3.33 even).}

$$
\begin{gathered}
\sum_{J, k, L \geqq 0}(-1)^{k+L} q^{2 J^{2}+k}\left[\begin{array}{c}
m-J+k-1 \\
k
\end{array}\right]_{q^{2}}\left[\begin{array}{c}
m-J+L-1 \\
L
\end{array}\right]_{q^{2}}\left[\begin{array}{c}
m+J-k-L \\
m-J
\end{array}\right]_{q^{2}} \\
=\sum_{k=-\infty}^{\infty} q^{42 k^{2}-2 k}\left[\begin{array}{c}
2 m \\
m+7 k
\end{array}\right]_{q^{2}}-q^{42 k^{2}+26 k+4}\left[\begin{array}{c}
2 m \\
m+7 k-2
\end{array}\right]_{q^{2}} \\
+q^{42 k^{2}+30 k+5}\left(\left[\begin{array}{c}
2 m \\
m+7 k+2
\end{array}\right]_{q^{2}}-\left[\begin{array}{c}
2 m \\
m+7 k+3
\end{array}\right]_{q^{2}}\right)
\end{gathered}
$$

Identity 5.33-even (Dual of A.33 even).

$$
\begin{gathered}
\sum_{J=0}^{\infty} \sum_{k=0}^{\infty} \sum_{L=0}^{\infty} \frac{(-1)^{k+L} q^{2 J^{2}+k}}{\left(q^{2} ; q^{2}\right)_{k}\left(q^{2} ; q^{2}\right)_{L}\left(q^{2} ; q^{2}\right)_{2 J-k-L}} \\
=\prod_{j=1}^{\infty} \frac{\left(1-q^{28 j-4}\right)\left(1-q^{28 j-24}\right)\left(1-q^{28 j}\right)\left(1-q^{56 j-20}\right)\left(1-q^{56 j-36}\right)}{\left(1-q^{2 j}\right)}
\end{gathered}
$$

Observation 5.34. Identity 34 is self-dual. 


\section{Identity 5.35f (Dual of 3.35).}

$$
\begin{gathered}
\sum_{h \geqq 0} \sum_{i \geqq 0} \sum_{k \geqq 0} q^{2 h(k+i)+h(h+3) / 2+4 i k+2 k(k+1)+3 i(i+1)}\left[\begin{array}{c}
n-2 i-h-2 k \\
i
\end{array}\right]_{q^{2}} \\
\quad \times\left[\begin{array}{c}
n-2 i-h-k \\
k
\end{array}\right]_{q^{2}}\left[\begin{array}{c}
n-2 i-2 k \\
h
\end{array}\right]_{q} \\
=\sum_{j=-\infty}^{\infty}(-1)^{j} q^{n+4 j^{2}+3 j+1}\left(\begin{array}{c}
n+1,4 j+1 ; q \\
4 j+2
\end{array}\right)_{2} \\
+\sum_{j=-\infty}^{\infty}(-1)^{j} q^{4 j^{2}+3 j}\left(\begin{array}{c}
n+1,4 j+1 ; q \\
4 j+1
\end{array}\right)_{2}
\end{gathered}
$$

Identity 5.35 (Dual of A.35).

$$
\begin{aligned}
\sum_{h=0}^{\infty} & \sum_{i=0}^{\infty} \sum_{k=0}^{\infty} \frac{q^{2 h(k+i)+h(h+3) / 2+4 i k+2 k(k+1)+3 i(i+1)}}{\left(q^{2} ; q^{2}\right)_{i}\left(q^{2} ; q^{2}\right)_{k}(q ; q)_{h}} \\
& =\prod_{j=1}^{\infty} \frac{\left(1-q^{8 j-1}\right)\left(1-q^{8 j-7}\right)\left(1-q^{8 j}\right)}{\left(1-q^{j}\right)}
\end{aligned}
$$

Observation 5.36. Identity 36 is self-dual.

Identity $5.37 \mathrm{f}$ (Dual of 3.37).

$$
\begin{gathered}
\sum_{h \geqq 0} \sum_{i \geqq 0} \sum_{k \geqq 0} q^{2 h k+h(h+1) / 2+4 i k+2 i h+2 k^{2}+3 i^{2}+i}\left[\begin{array}{c}
n-2 i-h-2 k \\
i
\end{array}\right]_{q^{2}}\left[\begin{array}{c}
n-2 i-h-k \\
k
\end{array}\right]_{q^{2}} \\
\times\left[\begin{array}{c}
n-2 i-2 k \\
h
\end{array}\right]_{q} \\
=\sum_{j=-\infty}^{\infty}(-1)^{j} q^{n+4 j^{2}+j}\left(\begin{array}{c}
n, 4 j ; q \\
4 j+1
\end{array}\right)_{2}+\sum_{j=-\infty}^{\infty}(-1)^{j} q^{4 j^{2}+j}\left(\begin{array}{c}
n, 4 j ; q \\
4 j
\end{array}\right)_{2}
\end{gathered}
$$

Identity 5.37 (Dual of A.37). Note: This identity is equivalent to $(-q ; q)_{\infty} \times(A .39)$ with $q$ replaced by $-q$.

$$
\sum_{h=0}^{\infty} \sum_{i=0}^{\infty} \sum_{k=0}^{\infty} \frac{q^{2 h k+h(h+1) / 2+4 i k+2 i h+2 k^{2}+3 i^{2}+i}}{\left(q^{2} ; q^{2}\right)_{i}\left(q^{2} ; q^{2}\right)_{k}(q ; q)_{h}}=\prod_{j=1}^{\infty} \frac{\left(1-q^{8 j-3}\right)\left(1-q^{8 j-5}\right)\left(1-q^{8 j}\right)}{\left(1-q^{j}\right)}
$$

Observation 5.38-even. Identity (38 even) is the dual of (39 odd).

Observation 5.38-odd. Identity (38 odd) is self-dual.

Observation 5.39-even. Identity (39 even) is self-dual.

Observation 5.39-odd. Identity (39 odd) is the dual of (38 even). 
Identity 5.45 (Dual of A.45).

$$
\begin{gathered}
\sum_{h=0}^{\infty} \sum_{i=0}^{\infty} \sum_{k=0}^{\infty} \frac{q^{2 k(i+h)+i h+h(h+1) / 2+2 k^{2}+i^{2}}}{\left(q^{2} ; q^{2}\right)_{i}\left(q^{2} ; q^{2}\right)_{k}(q ; q)_{h}} \\
=\prod_{j=1}^{\infty} \frac{\left(1+q^{5 j-1}\right)\left(1+q^{5 j-4}\right)\left(1-q^{10 j-3}\right)\left(1-q^{10 j-7}\right)\left(1-q^{5 j}\right)}{\left(1-q^{j}\right)}
\end{gathered}
$$

\section{Identity 5.50f (Dual of 3.50).}

$$
\begin{gathered}
\sum_{h \geqq 0} \sum_{i \geqq 0} q^{2 i^{2}+2 i h+h^{2}+2 i+h}\left[\begin{array}{c}
n-i-h \\
i
\end{array}\right]_{q^{2}}\left[\begin{array}{c}
2 n-2 i-h+1 \\
h
\end{array}\right]_{q} \\
=\sum_{j=-\infty}^{\infty}(-1)^{j} q^{3 j^{2}+2 j}\left[\begin{array}{c}
2 n+2 \\
n+3 j+2
\end{array}\right]_{q}
\end{gathered}
$$

Identity 5.50 (Dual of A.50).

$$
\sum_{h=0}^{\infty} \sum_{i=0}^{\infty} \frac{q^{(h+i)^{2}+i(i+2)+h}}{\left(q^{2} ; q^{2}\right)_{i}(q ; q)_{h}}=\prod_{j=1}^{\infty} \frac{\left(1-q^{6 j-1}\right)\left(1-q^{6 j-5}\right)\left(1-q^{6 j}\right)}{\left(1-q^{j}\right)}
$$

Identity 5.59f-even (Dual of 3.59 even).

$$
\begin{array}{r}
\sum_{j \geqq 0} \sum_{k \geqq 0} q^{(j+k)^{2}+2 j+k}\left[\begin{array}{c}
n-j \\
k
\end{array}\right]_{q^{2}}\left[\begin{array}{c}
n+j-k \\
2 j
\end{array}\right]_{q} \\
=\sum_{k=-\infty}^{\infty} q^{21 k^{2}+4 k}\left[\begin{array}{c}
2 m+2 \\
m+7 k+2
\end{array}\right]_{q}-q^{21 k^{2}+32 k+4}\left[\begin{array}{c}
2 m+2 \\
m+7 k+6
\end{array}\right]_{q}
\end{array}
$$

Identity 5.59 even (Dual of A.59 even). Note: This identity is equivalent to $\left(-q ; q^{2}\right)_{\infty} \times(A .118)$

$$
\begin{gathered}
\sum_{j=0}^{\infty} \sum_{k=0}^{\infty} \frac{q^{(j+k)^{2}+2 j+k}}{\left(q^{2} ; q^{2}\right)_{k}(q ; q)_{2 j}} \\
=\prod_{j=1}^{\infty} \frac{\left(1-q^{14 j-1}\right)\left(1-q^{14 j-13}\right)\left(1-q^{28 j-12}\right)\left(1-q^{28 j-16}\right)\left(1-q^{14 j}\right)}{\left(1-q^{j}\right)}
\end{gathered}
$$

Identity 5.59f odd (Dual of 3.59 odd).

$$
\begin{array}{r}
\sum_{j \geqq 0} \sum_{k \geqq 0} q^{(j+k)^{2}+3 j+2 k}\left[\begin{array}{c}
n-j \\
k
\end{array}\right]_{q^{2}}\left[\begin{array}{c}
n+j-k+1 \\
2 j+1
\end{array}\right]_{q} \\
=\sum_{k=-\infty}^{\infty} q^{21 k^{2}+11 k}\left[\begin{array}{c}
2 m+3 \\
m+7 k+3
\end{array}\right]_{q}-q^{21 k^{2}+25 k+6}\left[\begin{array}{c}
2 m+3 \\
m+7 k+6
\end{array}\right]_{q}
\end{array}
$$


Identity 5.59 odd (Dual of A.59 odd). Note: This identity is equivalent to $\left(-q^{2} ; q^{2}\right)_{\infty} \times\left(\right.$ A.82) with $q$ replaced by $q^{2}$.

$$
\begin{gathered}
\sum_{j=0}^{\infty} \sum_{k=0}^{\infty} \frac{q^{(j+k)^{2}+3 j+2 k}}{\left(q^{2} ; q^{2}\right)_{k}(q ; q)_{2 j+1}} \\
=\prod_{j=1}^{\infty} \frac{\left(1-q^{14 j-6}\right)\left(1-q^{14 j-8}\right)\left(1-q^{28 j-2}\right)\left(1-q^{28 j-26}\right)\left(1-q^{14 j}\right)}{\left(1-q^{j}\right)}
\end{gathered}
$$

Identity 5.60f-even (Dual of 3.60 even).

$$
\begin{array}{r}
\sum_{j \geqq 0} \sum_{k \geqq 0} q^{(j+k)^{2}+j}\left[\begin{array}{c}
n-j \\
k
\end{array}\right]_{q^{2}}\left[\begin{array}{c}
n+j-k \\
2 j
\end{array}\right]_{q} \\
=\sum_{k=-\infty}^{\infty} q^{21 k^{2}+k}\left[\begin{array}{c}
2 m+1 \\
m+7 k+1
\end{array}\right]_{q}-q^{21 k^{2}+29 k+10}\left[\begin{array}{c}
2 m+1 \\
m+7 k+5
\end{array}\right]_{q}
\end{array}
$$

Identity 5.60-even (Dual of A.60 even). Note: This identity is equivalent to $\left(-q ; q^{2}\right)_{\infty} \times(A .81)$ with $q$ replaced by $q^{2}$.

$$
\begin{gathered}
\sum_{j=0}^{\infty} \sum_{k=0}^{\infty} \frac{q^{(j+k)^{2}+j}}{\left(q^{2} ; q^{2}\right)_{k}(q ; q)_{2 j}} \\
=\prod_{j=1}^{\infty} \frac{\left(1-q^{14 j-2}\right)\left(1-q^{14 j-12}\right)\left(1-q^{28 j-10}\right)\left(1-q^{28 j-18}\right)\left(1-q^{14 j}\right)}{\left(1-q^{j}\right)}
\end{gathered}
$$

Identity 5.60f-odd (Dual of 3.60 odd).

$$
\begin{array}{r}
\sum_{j \geqq 0} \sum_{k \geqq 0} q^{(j+k)^{2}+2 j+k}\left[\begin{array}{c}
n-j \\
k
\end{array}\right]_{q^{2}}\left[\begin{array}{c}
n+j-k+1 \\
2 j+1
\end{array}\right]_{q} \\
=\sum_{k=-\infty}^{\infty} q^{21 k^{2}+8 k}\left[\begin{array}{c}
2 m+2 \\
m+7 k+2
\end{array}\right]_{q}-q^{21 k^{2}+22 k+5}\left[\begin{array}{c}
2 m+2 \\
m+7 k+5
\end{array}\right]_{q}
\end{array}
$$

Identity 5.60-odd (Dual of A.60 odd). Note: This identity is equivalent to $\left(-q ; q^{2}\right)_{\infty} \times(A .119)$

$$
\begin{gathered}
\sum_{j=0}^{\infty} \sum_{k=0}^{\infty} \frac{q^{(j+k)^{2}+2 j+k}}{\left(q^{2} ; q^{2}\right)_{k}(q ; q)_{2 j+1}}= \\
\prod_{j=1}^{\infty} \frac{\left(1-q^{14 j-5}\right)\left(1-q^{14 j-9}\right)\left(1-q^{28 j-4}\right)\left(1-q^{28 j-28}\right)\left(1-q^{14 j}\right)}{\left(1-q^{j}\right)}
\end{gathered}
$$




\section{Identity 5.61f-even (Dual of 3.61 even).}

$$
\begin{array}{r}
\sum_{j \geqq 0} \sum_{k \geqq 0} q^{(j+k)^{2}+k}\left[\begin{array}{c}
n-j-1 \\
k
\end{array}\right]_{q^{2}}\left[\begin{array}{c}
n+j-k \\
2 j
\end{array}\right]_{q} \\
=\sum_{k=-\infty}^{\infty} q^{21 k^{2}+2 k}\left[\begin{array}{c}
2 m \\
m+7 k
\end{array}\right]_{q}-q^{21 k^{2}+26 k+8}\left[\begin{array}{c}
2 m \\
m+7 k+4
\end{array}\right]_{q}
\end{array}
$$

Identity 5.61-even (Dual of A.61 even). Note: This identity is equivalent to $\left(-q^{2} ; q^{2}\right)_{\infty} \times(A .117)$.

$$
\begin{gathered}
\sum_{j=0}^{\infty} \sum_{k=0}^{\infty} \frac{q^{(j+k)^{2}+k}}{\left(q^{2} ; q^{2}\right)_{k}(q ; q)_{2 j}} \\
=\prod_{j=1}^{\infty} \frac{\left(1-q^{14 j-3}\right)\left(1-q^{14 j-11}\right)\left(1-q^{28 j-8}\right)\left(1-q^{28 j-20}\right)\left(1-q^{14 j}\right)}{\left(1-q^{j}\right)}
\end{gathered}
$$

Identity 5.61f-odd (Dual of 3.61 odd).

$$
\begin{array}{r}
\sum_{j \geqq 0} \sum_{k \geqq 0} q^{(j+k)^{2}+j+2 k}\left[\begin{array}{c}
n-j-1 \\
k
\end{array}\right]_{q^{2}}\left[\begin{array}{c}
n+j-k+1 \\
2 j+1
\end{array}\right]_{q} \\
=\sum_{k=-\infty}^{\infty} q^{21 k^{2}+5 k}\left[\begin{array}{c}
2 m \\
m+7 k+1
\end{array}\right]_{q}-q^{21 k^{2}+19 k+4}\left[\begin{array}{c}
2 m \\
m+7 k+4
\end{array}\right]_{q}
\end{array}
$$

Identity 5.61-odd (Dual of A.61 odd). Note: This identity is equivalent to $\left(-q ; q^{2}\right)_{\infty} \times\left(\right.$ A.80) with $q$ replaced by $q^{2}$.

$$
\begin{gathered}
\sum_{j=0}^{\infty} \sum_{k=0}^{\infty} \frac{q^{(j+k)^{2}+j+2 k}}{\left(q^{2} ; q^{2}\right)_{k}(q ; q)_{2 j+1}} \\
=\prod_{j=1}^{\infty} \frac{\left(1-q^{14 j-4}\right)\left(1-q^{14 j-10}\right)\left(1-q^{28 j-6}\right)\left(1-q^{28 j-22}\right)\left(1-q^{14 j}\right)}{\left(1-q^{j}\right)}
\end{gathered}
$$

Identity 5.68f (Dual of 3.68).

$$
\begin{gathered}
\sum_{h \geqq 0} \sum_{i \geqq 0} \sum_{k \geqq 0} \sum_{l \geqq 0}(-1)^{k} q^{2 h+2 i\left(2 h+2 k+2 l+1+3 i+(h+k)^{2}\right.}\left[\begin{array}{c}
n-2 i-h-k-l \\
i
\end{array}\right]_{q^{4}} \\
\times\left[\begin{array}{c}
n-2 i-h-l \\
k
\end{array}\right]_{q^{2}} \times\left[\begin{array}{c}
n-2 i-h-k \\
l
\end{array}\right]_{q^{2}}\left[\begin{array}{c}
n-2 i-k-l \\
h
\end{array}\right]_{q^{2}} \\
=\sum_{j=-\infty}^{\infty}(-1)^{j} q^{8 j^{2}+2 j}\left(\begin{array}{c}
n+1,4 j+1 ; q^{2} \\
4 j+1
\end{array}\right)_{2}-(-1)^{j} q^{8 j^{2}+10 j+3}\left(\begin{array}{c}
n+1,4 j+2 ; q^{2} \\
4 j+2
\end{array}\right)_{2}
\end{gathered}
$$


Identity 5.68 (Dual of A.68).

$$
\begin{gathered}
\sum_{h=0}^{\infty} \sum_{i=0}^{\infty} \sum_{k=0}^{\infty} \sum_{l=0}^{\infty} \frac{(-1)^{k} q^{2 h+2 i\left(2 h+2 k+2 l+1+3 i+(h+k)^{2}\right.}}{\left(q^{4} ; q^{4}\right)_{i}\left(q^{2} ; q^{2}\right)_{k}\left(q^{2} ; q^{2}\right)_{l}\left(q^{2} ; q^{2}\right)_{h}} \\
=\frac{\left(q,-q^{3},-q^{4} ;-q^{4}\right)_{\infty}}{\left(q^{2} ; q^{2}\right)_{\infty}}
\end{gathered}
$$

Identity 5.69f (Dual of 3.69).

$$
\begin{gathered}
\sum_{h \geqq 0} \sum_{i \geqq 0} \sum_{k \geqq 0} \sum_{l \geqq 0}(-1)^{i} q^{2 h+2 i(2 h+2 k+2 l+1+3 i)+(h+k)^{2}}\left[\begin{array}{c}
n-2 i-h-k-l \\
i
\end{array}\right]_{q^{4}} \\
\times\left[\begin{array}{c}
n-2 i-h-l \\
k
\end{array}\right]_{q^{2}} \times\left[\begin{array}{c}
n-2 i-h-k \\
l
\end{array}\right]_{q^{2}}\left[\begin{array}{c}
n-2 i-k-l \\
h
\end{array}\right]_{q^{2}} \\
=\sum_{j=-\infty}^{\infty}(-1)^{j} q^{8 j^{2}+2 j}\left(\begin{array}{c}
n+1,4 j+1 ; q^{2} \\
4 j+1
\end{array}\right)_{2}+(-1)^{j} q^{8 j^{2}+10 j+3}\left(\begin{array}{c}
n+1,4 j+2 ; q^{2} \\
4 j+2
\end{array}\right)_{2}
\end{gathered}
$$

Identity 5.69 (Dual of A.69).

$$
\sum_{h=0}^{\infty} \sum_{i=0}^{\infty} \sum_{k=0}^{\infty} \sum_{l=0}^{\infty} \frac{(-1)^{i} q^{2 j+2 i(2 h+2 k+2 l+1+3 i)+(h+k)^{2}}}{\left(q^{4} ; q^{4}\right)_{i}\left(q^{2} ; q^{2}\right)_{k}\left(q^{2} ; q^{2}\right)_{l}\left(q^{2} ; q^{2}\right)_{h}}=\prod_{j=1}^{\infty}\left(1+q^{j}\right)
$$

Observation 5.79. The dual of (79) is (18 even).

Identity 5.80f (Dual of 3.80).

$$
\begin{gathered}
\sum_{h \geqq 0} \sum_{k \geqq 0} q^{h(h+1) / 2+2 k(h+k)}\left[\begin{array}{c}
n-h-k \\
k
\end{array}\right]_{q^{2}}\left[\begin{array}{c}
n-2 k \\
h
\end{array}\right]_{q} \\
=\sum_{k=-\infty}^{\infty} q^{14 k^{2}+k}\left(\begin{array}{c}
n+1,7 k ; q \\
7 k+1
\end{array}\right)_{2}-q^{14 k^{2}+13 k+3}\left(\begin{array}{c}
n+1,7 k+3 ; q \\
7 k+4
\end{array}\right)_{2}
\end{gathered}
$$

Identity 5.80 (Dual of A.80). Equivalent to Andrews [5, p. 331, eqn. (1.1)], and to $(-q ; q)_{\infty} \times(A .33)$.

$$
\sum_{h=0}^{\infty} \sum_{k=0}^{\infty} \frac{q^{h(h+1) / 2+2 k(h+k)}}{\left(q^{2} ; q^{2}\right)_{k}(q ; q)_{h}}=\prod_{j=1}^{\infty} \frac{\left(1-q^{7 j-3}\right)\left(1-q^{7 j-4}\right)\left(1-q^{7 j}\right)}{\left(1-q^{j}\right)}
$$

Identity 5.81f (Dual of 3.81).

$$
\begin{array}{r}
\sum_{h \geqq 0} \sum_{k \geqq 0} q^{h(h+1) / 2+2 k(h+k+1)}\left[\begin{array}{c}
n-h-k-1 \\
k
\end{array}\right]_{q^{2}}\left[\begin{array}{c}
n-2 k \\
h
\end{array}\right]_{q} \\
=\sum_{k=-\infty}^{\infty} q^{14 k^{2}-4 k}\left(\begin{array}{c}
n+1,7 k-1 ; q \\
7 k
\end{array}\right)_{2}-q^{14 k^{2}+4 k}\left(\begin{array}{c}
n+1,7 k+1 ; q \\
7 k+2
\end{array}\right)_{2}
\end{array}
$$


Identity 5.81 (Dual of A.81). Equivalent to Andrews [5, p. 331, eqn. (1.2)], and to $(-q ; q)_{\infty} \times(A .32)$.

$$
\sum_{h=0}^{\infty} \sum_{k=0}^{\infty} \frac{q^{h(h+1) / 2+2 k(h+k+1)}}{\left(q^{2} ; q^{2}\right)_{k}(q ; q)_{h}}=\prod_{j=1}^{\infty} \frac{\left(1-q^{7 j-2}\right)\left(1-q^{7 j-5}\right)\left(1-q^{7 j}\right)}{\left(1-q^{j}\right)}
$$

Identity 5.82f (Dual of 3.82).

$$
\begin{gathered}
\sum_{h \geqq 0} \sum_{k \geqq 0} q^{h(h+3) / 2+2 k(h+k+1)}\left[\begin{array}{c}
n-h-k \\
k
\end{array}\right]_{q^{2}}\left[\begin{array}{c}
n-2 k \\
h
\end{array}\right]_{q} \\
=\sum_{k=-\infty}^{\infty} q^{14 k^{2}+5 k}\left(\begin{array}{c}
n+2,7 k+1 ; q \\
7 k+2
\end{array}\right)_{2}-q^{14 k^{2}+9 k+1}\left(\begin{array}{c}
n+2,7 k+2 ; q \\
7 k+3
\end{array}\right)_{2}
\end{gathered}
$$

Identity 5.82 (Dual of A.82). Equivalent to Andrews [5, p. 331, eqn. (1.3)], and to $(-q ; q)_{\infty} \times(A .31)$.

$$
\sum_{h=0}^{\infty} \sum_{k=0}^{\infty} \frac{q^{h(h+3) / 2+2 k(h+k+1)}}{\left(q^{2} ; q^{2}\right)_{k}(q ; q)_{h}}=\prod_{j=1}^{\infty} \frac{\left(1-q^{7 j-1}\right)\left(1-q^{7 j-6}\right)\left(1-q^{7 j}\right)}{\left(1-q^{j}\right)}
$$

Identity 5.90f-even (Dual of 3.90-even).

$$
\begin{gathered}
\sum_{i=0}^{\infty} \sum_{J=0}^{\infty} \sum_{k=0}^{\infty} \sum_{L=0}^{\infty}(-1)^{i} q^{J^{2}+3 J+3 i(i-1) / 2-k-2 L}\left[\begin{array}{c}
n-J \\
i
\end{array}\right]_{q^{3}} \\
\times\left[\begin{array}{c}
n-J+k \\
k
\end{array}\right]_{q^{2}}\left[\begin{array}{c}
n-J+L \\
L
\end{array}\right]_{q^{2}}\left[\begin{array}{c}
n+J-3 i-2 k-2 L \\
n-J
\end{array}\right]_{q} \\
=\sum_{k=-\infty}^{\infty} q^{27 k^{2}+6 k}\left[\begin{array}{c}
2 m+3 \\
m+9 k+3
\end{array}\right]_{q}-q^{27 k^{2}+42 k+16}\left[\begin{array}{c}
2 m+3 \\
m+9 k+8
\end{array}\right]_{q}
\end{gathered}
$$

Identity 5.90-even (Dual of A.90-even).

$$
\begin{aligned}
& \sum_{i=0}^{\infty} \sum_{J=0}^{\infty} \sum_{k=0}^{\infty} \sum_{L=0}^{\infty} \frac{(-1)^{i} q^{J^{2}+3 J+3 i(i-1) / 2-k-2 L}}{\left(q^{3} ; q^{3}\right)_{i}\left(q^{2} ; q^{2}\right)_{k}\left(q^{2} ; q^{2}\right)_{L}(q ; q)_{2 J-3 i-2 k-2 L}} \\
= & \prod_{j=1}^{\infty} \frac{\left(1-q^{18 j-1}\right)\left(1-q^{18 j-17}\right)\left(1-q^{18 j}\right)\left(1-q^{36 j-16}\right)\left(1-q^{36 j-20}\right)}{\left(1-q^{j}\right)}
\end{aligned}
$$

Identity 5.90f-odd (Dual of 3.90-odd).

$$
\begin{array}{r}
\sum_{i=0}^{\infty} \sum_{J=0}^{\infty} \sum_{k=0}^{\infty} \sum_{L=0}^{\infty}(-1)^{i} q^{J^{2}+4 J+3 i(i-1) / 2-k-2 L}\left[\begin{array}{c}
n-J \\
i
\end{array}\right]_{q^{3}} \\
\times\left[\begin{array}{c}
n-J+k \\
k
\end{array}\right]_{q^{2}}\left[\begin{array}{c}
n-J+L \\
L
\end{array}\right]_{q^{2}}\left[\begin{array}{c}
n+J-3 i-2 k-2 L \\
n-J
\end{array}\right]_{q} \\
=\sum_{k=-\infty}^{\infty} q^{27 k^{2}+15 k}\left[\begin{array}{c}
2 m+4 \\
m+9 k+4
\end{array}\right]_{q}-q^{27 k^{2}+33 k+8}\left[\begin{array}{c}
2 m+4 \\
m+9 k+8
\end{array}\right]_{q}
\end{array}
$$




\section{Identity 5.90-odd (Dual of A.90-odd).}

$$
\begin{aligned}
& \sum_{i=0}^{\infty} \sum_{J=0}^{\infty} \sum_{k=0}^{\infty} \sum_{L=0}^{\infty} \frac{(-1)^{i} q^{J^{2}+4 J+3 i(i-1) / 2-k-2 L}}{\left(q^{3} ; q^{3}\right)_{i}\left(q^{2} ; q^{2}\right)_{k}\left(q^{2} ; q^{2}\right)_{L}(q ; q)_{2 J-3 i-2 k-2 L}} \\
= & \prod_{j=1}^{\infty} \frac{\left(1-q^{18 j-8}\right)\left(1-q^{18 j-10}\right)\left(1-q^{18 j}\right)\left(1-q^{36 j-2}\right)\left(1-q^{36 j-34}\right)}{\left(1-q^{j}\right)}
\end{aligned}
$$

\section{Identity 5.91f-even (Dual of 3.91-even).}

$$
\begin{array}{r}
\sum_{i=0}^{\infty} \sum_{J=0}^{\infty} \sum_{k=0}^{\infty} \sum_{L=0}^{\infty}(-1)^{i} q^{J^{2}+2 J+3 i(i-1) / 2-k-2 L}\left[\begin{array}{c}
n-J \\
i
\end{array}\right]_{q^{3}} \\
\times\left[\begin{array}{c}
n-J+k \\
k
\end{array}\right]_{q^{2}}\left[\begin{array}{c}
n-J+L \\
L
\end{array}\right]_{q^{2}}\left[\begin{array}{c}
n+J-3 i-2 k-2 L \\
n-J
\end{array}\right]_{q} \\
=\sum_{k=-\infty}^{\infty} q^{27 k^{2}+3 k}\left[\begin{array}{c}
2 m+2 \\
m+9 k+2
\end{array}\right]_{q}-q^{27 k^{2}+39 k+14}\left[\begin{array}{c}
2 m+2 \\
m+9 k+7
\end{array}\right]_{q}
\end{array}
$$

Identity 5.91-even (Dual of A.91-even).

$$
\begin{array}{r}
\sum_{i=0}^{\infty} \sum_{J=0}^{\infty} \sum_{k=0}^{\infty} \sum_{L=0}^{\infty} \frac{(-1)^{i} q^{J^{2}+2 J+3 i(i-1) / 2-k-2 L}}{\left(q^{3} ; q^{3}\right)_{i}\left(q^{2} ; q^{2}\right)_{k}\left(q^{2} ; q^{2}\right)_{L}(q ; q)_{2 J-3 i-2 k-2 L}} \\
=\prod_{j=1}^{\infty} \frac{\left(1-q^{18 j-2}\right)\left(1-q^{18 j-16}\right)\left(1-q^{18 j}\right)\left(1-q^{36 j-14}\right)\left(1-q^{36 j-22}\right)}{\left(1-q^{j}\right)}
\end{array}
$$

Identity 5.91f-odd (Dual of 3.91-odd).

$$
\begin{gathered}
\sum_{i=0}^{\infty} \sum_{J=0}^{\infty} \sum_{k=0}^{\infty} \sum_{L=0}^{\infty}(-1)^{i} q^{J^{2}+3 J+3 i(i-1) / 2-k-2 L}\left[\begin{array}{c}
n-J \\
i
\end{array}\right]_{q^{3}} \\
\times\left[\begin{array}{c}
n-J+k \\
k
\end{array}\right]_{q^{2}}\left[\begin{array}{c}
n-J+L \\
L
\end{array}\right]_{q^{2}}\left[\begin{array}{c}
n+J+1-3 i-2 k-2 L \\
n-J
\end{array}\right]_{q} \\
=\sum_{k=-\infty}^{\infty} q^{27 k^{2}+12 k}\left[\begin{array}{c}
2 m+3 \\
m+9 k+3
\end{array}\right]_{q}-q^{27 k^{2}+30 k+7}\left[\begin{array}{c}
2 m+3 \\
m+9 k+7
\end{array}\right]_{q}
\end{gathered}
$$

Identity 5.91-odd (Dual of A.91-odd).

$$
\begin{array}{r}
\sum_{i=0}^{\infty} \sum_{J=0}^{\infty} \sum_{k=0}^{\infty} \sum_{L=0}^{\infty} \frac{(-1)^{i} q^{J^{2}+3 J+3 i(i-1) / 2-k-2 L}}{\left(q^{3} ; q^{3}\right)_{i}\left(q^{2} ; q^{2}\right)_{k}\left(q^{2} ; q^{2}\right)_{L}(q ; q)_{2 J-3 i-2 k-2 L}} \\
=\prod_{j=1}^{\infty} \frac{\left(1-q^{18 j-7}\right)\left(1-q^{18 j-11}\right)\left(1-q^{18 j}\right)\left(1-q^{36 j-4}\right)\left(1-q^{36 j-32}\right)}{\left(1-q^{j}\right)}
\end{array}
$$


Identity 5.92f-even (Dual of 3.92-even).

$$
\begin{gathered}
\sum_{i=0}^{\infty} \sum_{J=0}^{\infty} \sum_{k=0}^{\infty} \sum_{L=0}^{\infty}(-1)^{i} q^{J^{2}+J+3 i(i-1) / 2-k}\left[\begin{array}{c}
n-J \\
i
\end{array}\right]_{q^{3}} \\
\times\left[\begin{array}{c}
n-J+k \\
k
\end{array}\right]_{q^{2}}\left[\begin{array}{c}
n-J+L-1 \\
L
\end{array}\right]_{q^{2}}\left[\begin{array}{c}
n+J-3 i-2 k-2 L \\
n-J
\end{array}\right]_{q} \\
=\sum_{k=-\infty}^{\infty} q^{27 k^{2}+3 k}\left[\begin{array}{c}
2 m+2 \\
m+9 k+2
\end{array}\right]_{q}-q^{27 k^{2}+39 k+14}\left[\begin{array}{c}
2 m+2 \\
m+9 k+7
\end{array}\right]_{q}
\end{gathered}
$$

Identity 5.92-even (Dual of A.92-even).

$$
\begin{array}{r}
\sum_{i=0}^{\infty} \sum_{J=0}^{\infty} \sum_{k=0}^{\infty} \sum_{L=0}^{\infty} \frac{(-1)^{i} q^{J^{2}+J+3 i(i-1) / 2-k}}{\left(q^{3} ; q^{3}\right)_{i}\left(q^{2} ; q^{2}\right)_{k}\left(q^{2} ; q^{2}\right)_{L}(q ; q)_{2 J-3 i-2 k-2 L}} \\
=\prod_{j=1}^{\infty} \frac{\left(1-q^{18 j-3}\right)\left(1-q^{18 j-15}\right)\left(1-q^{18 j}\right)\left(1-q^{36 j-12}\right)\left(1-q^{36 j-24}\right)}{\left(1-q^{j}\right)}
\end{array}
$$

Identity 5.92f-odd (Dual of 3.92-odd).

$$
\begin{gathered}
\sum_{i=0}^{\infty} \sum_{J=0}^{\infty} \sum_{k=0}^{\infty} \sum_{L=0}^{\infty}(-1)^{i} q^{J^{2}+2 J+3 i(i-1) / 2-k}\left[\begin{array}{c}
n-J \\
i
\end{array}\right]_{q^{3}} \\
\times\left[\begin{array}{c}
n-J+k \\
k
\end{array}\right]_{q^{2}}\left[\begin{array}{c}
n-J+L-1 \\
L
\end{array}\right]_{q^{2}}\left[\begin{array}{c}
n+J+1-3 i-2 k-2 L \\
n-J
\end{array}\right]_{q} \\
=\sum_{k=-\infty}^{\infty} q^{27 k^{2}+9 k}\left[\begin{array}{c}
2 m+2 \\
m+9 k+2
\end{array}\right]_{q}-q^{27 k^{2}+27 k+6}\left[\begin{array}{c}
2 m+2 \\
m+9 k+6
\end{array}\right]_{q}
\end{gathered}
$$

Identity 5.92-odd (Dual of A.92-odd).

$$
\begin{array}{r}
\sum_{i=0}^{\infty} \sum_{J=0}^{\infty} \sum_{k=0}^{\infty} \sum_{L=0}^{\infty} \frac{(-1)^{i} q^{J^{2}+2 J+3 i(i-1) / 2-k}}{\left(q^{3} ; q^{3}\right)_{i}\left(q^{2} ; q^{2}\right)_{k}\left(q^{2} ; q^{2}\right)_{L}(q ; q)_{2 J-3 i-2 k-2 L}} \\
=\prod_{j=1}^{\infty} \frac{\left(1-q^{18 j-6}\right)\left(1-q^{18 j-12}\right)\left(1-q^{18 j}\right)\left(1-q^{36 j-6}\right)\left(1-q^{36 j-30}\right)}{\left(1-q^{j}\right)}
\end{array}
$$

Observation 5.94. The dual of (94) is (18 odd).

Observation 5.96. The dual of (96) is (14 odd).

Observation 5.99. The dual of (99) is (14 even). 


\section{Identity 5.120f (Dual of 3.120).}

$$
\begin{gathered}
\sum_{h \geqq 0} \sum_{i \geqq 0} \sum_{k \geqq 0} q^{(h+k)^{2}+2 i(h+k+i)+h+2(k+i)}\left[\begin{array}{c}
n-i-h-k-1 \\
i
\end{array}\right]_{q^{2}}\left[\begin{array}{c}
n-i-h-1 \\
k
\end{array}\right]_{q^{2}} \\
\times\left[\begin{array}{c}
n-i-k \\
h
\end{array}\right]_{q^{2}} \\
=\sum_{j=-\infty}^{\infty} q^{12 j^{2}+4 j}\left[\begin{array}{c}
2 n+1 \\
n+6 j+1
\end{array}\right]_{q}-q^{12 j^{2}+8 j+1}\left[\begin{array}{c}
2 n+1 \\
n+6 j+2
\end{array}\right]_{q}
\end{gathered}
$$

Identity 5.120 (Dual of A.120).

$$
\sum_{h \geqq 0} \sum_{i \geqq 0} \sum_{k \geqq 0} \frac{q^{(h+k)^{2}+2 i(h+k+i)+h+2(k+i)}}{\left(q^{2} ; q^{2}\right)_{h}\left(q^{2} ; q^{2}\right)_{i}\left(q^{2} ; q^{2}\right)_{k}}=\prod_{j=1}^{\infty} \frac{\left(1-q^{6 j-1}\right)\left(1-q^{6 j-5}\right)\left(1-q^{6 j}\right)}{1-q^{j}}
$$

\section{Identity 5.130f (Dual of 3.130).}

$$
\begin{gathered}
\sum_{h=0}^{\infty} \sum_{i=0}^{\infty} \sum_{k=0}^{\infty} \sum_{l=0}^{\infty}(-1)^{l} q^{4 i k+4 i h+4 i l+h^{2}+2 h k+2 h l+l^{2}+2 k l+k^{2}-k+6 i^{2}}\left[\begin{array}{c}
n-2 i-h-k-l \\
i
\end{array}\right]_{q^{4}} \\
\times\left[\begin{array}{c}
n-2 i-h-l \\
k
\end{array}\right]_{q^{2}}\left[\begin{array}{c}
n-2 i-h-j-1 \\
l
\end{array}\right]_{q^{2}}\left[\begin{array}{c}
n-2 i-k-l \\
h
\end{array}\right]_{q^{2}} \\
=\sum_{j=-\infty}^{\infty}(-1)^{j} q^{8 j^{2}+4 j}\left(\begin{array}{c}
n, 4 j+1 ; q^{2} \\
4 j+1
\end{array}\right)_{2}+\sum_{j=-\infty}^{\infty}(-1)^{j} q^{8 j^{2}+4 j+2 n-1}\left(\begin{array}{c}
n-1,4 j+1 ; q^{2} \\
4 j+1
\end{array}\right)_{2} \\
\quad+\sum_{j=-\infty}^{\infty}(-1)^{j} q^{8 j^{2}}\left(\begin{array}{c}
n, 4 j ; q^{2} \\
4 j
\end{array}\right)_{2}+\sum_{j=-\infty}^{\infty}(-1)^{j} q^{8 j^{2}+2 n-1}\left(\begin{array}{c}
n-1,4 j ; q^{2} \\
4 j
\end{array}\right)_{2}
\end{gathered}
$$

\section{Identity 5.130 (Dual of A.130).}

$$
\begin{aligned}
& \sum_{h=0}^{\infty} \sum_{i=0}^{\infty} \sum_{k=0}^{\infty} \sum_{l=0}^{\infty}(-1)^{l} \frac{q^{4 i k+4 i h+4 i l+h^{2}+2 h k+2 h l+l^{2}+2 k l+k^{2}-k+6 i^{2}}}{\left(q^{4} ; q^{4}\right)_{i}\left(q^{2} ; q^{2}\right)_{k}\left(q^{2} ; q^{2}\right)_{l}\left(q^{2} ; q^{2}\right)_{h}} \\
= & \prod_{j=1}^{\infty} \frac{\left(1-q^{16 j-8}\right)^{2}\left(1-q^{16 j}\right)+\left(1-q^{16 j-4}\right)\left(1-q^{16 j-12}\right)\left(1-q^{16 j}\right)}{\left(1-q^{2 j}\right)}
\end{aligned}
$$




\section{More Finitizations: Bressoud Type Polynomial Iden- tities}

\subsection{On Bressoud's Identities}

The process of finitization is certainly not unique. There may be many different polynomial sequences which converge to a given series. So far, we have only considered the polynomial identites which arise when the two-parameter generalization $f(q, t)$ of the series $\phi(q)$ satisfies a first order, nonhomogeneous $q$-difference equation. For example, if we let

$$
\phi(q)=\sum_{j=0}^{\infty} \frac{q^{j^{2}}}{(q ; q)_{j}}
$$

the series side of the first Rogers-Ramanujan identity, equation (A.18), then

$$
\begin{array}{rlr}
f(q, t) & =\sum_{j=0}^{\infty} \frac{t^{2 j} q^{j^{2}}}{(t ; q)_{j+1}} & \\
& =\sum_{j=0}^{\infty} t^{2 j} q^{j^{2}} \sum_{k=0}^{\infty}\left[\begin{array}{c}
j+k \\
k
\end{array}\right]_{q} \quad \text { (by (1.11)) } \\
& =\sum_{j=0}^{\infty} \sum_{k=0}^{\infty} t^{2 j+k} q^{j^{2}}\left[\begin{array}{c}
j+k \\
k
\end{array}\right]_{q} & \\
& \left.=\sum_{n=0}^{\infty} t^{n} \sum_{k=0}^{\infty} q^{j^{2}}\left[\begin{array}{c}
n-j \\
k
\end{array}\right]_{q} \quad \text { (by letting } n=2 j+k\right) .
\end{array}
$$

So $f(q, t)$ is the generating function for polynomials $D_{n}(q)$ which are given by the identity

$$
\sum_{j \geqq 0} q^{j^{2}}\left[\begin{array}{c}
n-j \\
j
\end{array}\right]_{q}=\sum_{j=-\infty}^{\infty}(-1)^{j} q^{j(5 j+1) / 2}\left[\begin{array}{c}
n \\
\left\lfloor\frac{n+5 j+1}{2}\right\rfloor
\end{array}\right]_{q} .
$$

Let us examine what can happen if we drop the requirement that $f(q, t)$ satisfy the a first order nonhomogeneous $q$-difference equation, but retain the other two requirements of Conditions 2.1. We modify $f(q, t)$ slightly to obtain

$$
\begin{array}{rlr}
\tilde{f}(q, t) & =\sum_{j=0}^{\infty} \frac{t^{j} q^{j^{2}}}{(t ; q)_{j+1}} \\
& =\sum_{j=0}^{\infty} t^{j} q^{j^{2}} \sum_{k=0}^{\infty}\left[\begin{array}{c}
j+k \\
k
\end{array}\right]_{q} t^{k} & \quad(\text { by }(1.11)) \\
& =\sum_{j=0}^{\infty} \sum_{k=0}^{\infty} t^{j+k} q^{j^{2}}\left[\begin{array}{c}
j+k \\
k
\end{array}\right]_{q} \\
& \left.=\sum_{n=0}^{\infty} t^{n} \sum_{k=0}^{\infty} q^{j^{2}}\left[\begin{array}{c}
n \\
k
\end{array}\right]_{q} \quad \text { (by letting } n=j+k\right) .
\end{array}
$$


It turns out that $\tilde{f}(q, t)$ is the generating function for polynomials $B_{n}(q)$ which satisfy the polynomial identity

$$
\sum_{j \geqq 0} q^{j^{2}}\left[\begin{array}{l}
n \\
j
\end{array}\right]_{q}=\sum_{j=-\infty}^{\infty}(-1)^{j} q^{j(5 j+1) / 2}\left[\begin{array}{c}
2 n \\
n+2 j
\end{array}\right]_{q}
$$

an identity which was discovered by David Bressoud [32, p. 211, eqn. (1.1)] (by a different method), and is easily seen to converge to the first Rogers-Ramanujan identity (A.18). Note well that (6.1) is not merely an alternate representation of the MacMahon-Schur finitization (3.18) of the first Rogers-Ramanujan identity (A.18), but rather a different sequence of polynomials which also converges to (A.18).

We can perform the analagous calculation with the second Rogers-Ramanujan identity and arrive at

$$
\sum_{j \geqq 0} q^{j(j+1)}\left[\begin{array}{l}
n \\
j
\end{array}\right]_{q}=\sum_{j=-\infty}^{\infty}(-1)^{j} q^{j(5 j+3) / 2}\left[\begin{array}{c}
2 n+1 \\
n+2 j+1
\end{array}\right]_{q},
$$

which is equivalent to Bressoud [32, p. 212, eqn. (1.3)].

The next identity to appear in Bressoud's paper,

$$
\sum_{j \geqq 0} q^{j n}\left[\begin{array}{l}
n \\
j
\end{array}\right]_{q}=\sum_{j=-\infty}^{\infty}(-1)^{j} q^{j(3 j+1) / 2}\left[\begin{array}{c}
2 n \\
n+2 j
\end{array}\right]_{q}
$$

(Bressoud [32, p. 212, (1.5)]) is a finitization of Euler's Pentagonal Number Theorem (A.1). It is interesting to note that this identity is the reciprocal dual of both (6.1) and (6.2). This exemplifies the fact that duality can only be discussed within the context of a particular finitization. Recall from $\S 5$ that the dual of the First Rogers-Ramanujan Identity is the pair of identities (A.79) and (A.94). We see here, however, that under the Bressoud finitization of the First Rogers-Ramanujan Identity, its dual is Euler's Pentagonal Number Theorem. Thus, a reciprocal duality theory for Rogers-Ramanujan type identities only makes sense with respect to a given method of finitization.

\subsection{New Bressoud Type Polynomial Identities}

By altering the exponent of $t$ in the $f(q, t)$ associated with a given Rogers-Ramanujan type identity, we can always produce a fermionic representation for a sequence of polynomials $P_{n}(q)$ which converges to the series side of the original identity. In some cases, we may be lucky enough to find a nice bosonic representation for $P_{n}(q)$, and when this is the case, we have found what I will refer to as a polynomial identity of the Bressoud Type. Note, however, that since we have forfeited the generalized first order $q$-difference equation property, we no longer immediately obtain a recurrence relation for the polynomials generated. However, Riese's Mathematica packages can find a recurrence which the polyomials satisfy.

I found the following alternate finitizations of some of the identities on Slater's list. 
Identity 6.79 (Alternate finitization of (A.79)). This identity is explicitly a special case of Warnaar [71, equation (4.7)]. It is likely that the identities below can also be derived by the methods of Warnaar [71].

$$
\sum_{j \geqq 0} q^{j^{2}}\left[\begin{array}{c}
n \\
2 j
\end{array}\right]_{q}=\sum_{k=-\infty}^{\infty} q^{15 k^{2}+k}\left(\begin{array}{c}
n, 6 k ; q \\
6 k
\end{array}\right)_{2}-q^{15 k^{2}+19 k+6}\left(\begin{array}{c}
n, 6 k+4 ; q \\
6 k+4
\end{array}\right)_{2}
$$

Identity 6.94 (Alternative finitization of (A.94)).

$$
\begin{gathered}
\sum_{j \geqq 0} q^{j(j+1)}\left[\begin{array}{c}
n+1 \\
2 j+1
\end{array}\right]_{q} \\
=\sum_{k=-\infty}^{\infty} q^{15 k^{2}+4 k}\left(\begin{array}{c}
n+1,6 k+1 ; q \\
6 k+1
\end{array}\right)_{2}-q^{15 k^{2}+16 k+4}\left(\begin{array}{c}
n+1,6 k+3 ; q \\
6 k+3
\end{array}\right)_{2}
\end{gathered}
$$

Identity 6.96 (Alternative finitization of (A.96)).

$$
\begin{gathered}
\sum_{j \geqq 0} q^{j(j+2)}\left[\begin{array}{c}
n+1 \\
2 j+1
\end{array}\right]_{q} \\
=\sum_{k=-\infty}^{\infty} q^{15 k^{2}+7 k}\left(\begin{array}{c}
n+1,6 k+1 ; q \\
6 k+1
\end{array}\right)_{2}-q^{15 k^{2}+17 k+4}\left(\begin{array}{c}
n+1,6 k+3 ; q \\
6 k+3
\end{array}\right)_{2}
\end{gathered}
$$

\section{Identity 6.99 (Alternative finitization of (A.99)).}

$$
\sum_{j \geqq 0} q^{j(j+1)}\left[\begin{array}{c}
n \\
2 j
\end{array}\right]_{q}=\sum_{k=-\infty}^{\infty} q^{15 k^{2}+2 k}\left(\begin{array}{c}
n, 6 k ; q \\
6 k
\end{array}\right)_{2}-q^{15 k^{2}+22 k+8}\left(\begin{array}{c}
n, 6 k+4 ; q \\
6 k+4
\end{array}\right)_{2}
$$

Proof. We shall prove (6.79) and (6.96) simultaneously. Let $S_{n}(q)$ represent the polynomial on the LHS of (6.79), and $T_{n}(q)$ present the polynomial on the LHS of (6.96). Then

$$
\begin{array}{r}
T_{n}(q)=\sum_{j=0}^{\infty} q^{j(j+2)}\left[\begin{array}{c}
n+1 \\
2 j+1
\end{array}\right]_{q} \\
=\sum_{j=0}^{\infty} q^{j(j+2)}\left[\begin{array}{c}
n \\
2 j+1
\end{array}\right]_{q}+\sum_{j=0}^{\infty} q^{j^{2}+n}\left[\begin{array}{c}
n \\
2 j
\end{array}\right]_{q} \\
=T_{n-1}(q)+q^{n} S_{n}(q)
\end{array}
$$

Thus, we have

$$
T_{n}(q)-T_{n-1}(q)-q^{n} S_{n}(q)=0 \text { for } n \geqq 1 .
$$


Also,

$$
\begin{gathered}
S_{n}(q)=\sum_{j=0}^{\infty} q^{j^{2}}\left[\begin{array}{c}
n \\
2 j
\end{array}\right]_{q} \\
=\sum_{j=0}^{\infty} q^{j^{2}}\left[\begin{array}{c}
n-1 \\
2 j
\end{array}\right]_{q}+\sum_{j=1}^{\infty} q^{j^{2}+n-2 j}\left[\begin{array}{c}
n-1 \\
2 j-1
\end{array}\right]_{q} \quad(\text { by } 1.5) \\
=\sum_{j=0}^{\infty} q^{j^{2}}\left[\begin{array}{c}
n-1 \\
2 j
\end{array}\right]_{q}+q^{n-1} \sum_{j=0}^{\infty} q^{j^{2}}\left[\begin{array}{c}
n-1 \\
2 j+1
\end{array}\right]_{q} \\
=\sum_{j=0}^{\infty} q^{j^{2}}\left[\begin{array}{c}
n-1 \\
2 j
\end{array}\right]_{q}+q^{n-1} \sum_{j=0}^{\infty} q^{j^{2}}\left[\begin{array}{c}
n-2 \\
2 j
\end{array}\right]_{q}+q^{n} \sum_{j=0}^{\infty} q^{j(j-2)}\left[\begin{array}{c}
n-2 \\
2 j+1
\end{array}\right]_{q} \\
=S_{n-1}(q)+q^{n-1} S_{n-2}(q)+q^{n} T_{n-3}(q)
\end{gathered}
$$

Thus,

$$
S_{n}(q)-S_{n-1}(q)-q^{n-1} S_{n-2}(q)-q^{n} T_{n-3}(q)=0 \text { for } n \geqq 2 .
$$

So, by verifying that the RHS of (6.79) and (6.96) satisfy (6.4) and (6.5) along with the initial conditions

$$
S_{0}(q)=S_{1}(q)=T_{0}(q)=1,
$$

the identities will be proved.

We show that the RHS of (6.79) and (6.96) satisfy (6.4):

$$
\begin{gathered}
\sum_{j=-\infty}^{\infty} q^{15 j^{2}+7 j}\left(\begin{array}{c}
n+1,6 j+1 ; q \\
6 j+1
\end{array}\right)_{2}-\sum_{j=-\infty}^{\infty} q^{15 j^{2}+7 j}\left(\begin{array}{c}
n, 6 j+1 ; q \\
6 j+1
\end{array}\right)_{2} \\
-\sum_{j=-\infty}^{\infty} q^{15 j^{2}+17 j+4}\left(\begin{array}{c}
n+1,6 j+3 ; q \\
6 j+3
\end{array}\right)_{2}+\sum_{j=-\infty}^{\infty} q^{15 j^{2}+17 j+4}\left(\begin{array}{c}
n, 6 j+3 ; q \\
6 j+3
\end{array}\right)_{2} \\
-\sum_{j=-\infty}^{\infty} q^{15 j^{2}+j+n}\left(\begin{array}{c}
n, 6 j ; q \\
6 j
\end{array}\right)_{2}+\sum_{j=-\infty}^{\infty} q^{15 j^{2}+19 j+6+n}\left(\begin{array}{c}
n, 6 j+4 ; q \\
6 j+4
\end{array}\right)_{2}
\end{gathered}
$$

Apply (1.29) to the first and third terms to obtain

$$
\begin{gathered}
=\sum_{j=-\infty}^{\infty} q^{15 j^{2}+7 j+n}\left(\begin{array}{c}
n, 6 j+1 ; q \\
6 j+2
\end{array}\right)_{2}-\sum_{j=-\infty}^{\infty} q^{15 j^{2}+17 j+4+n}\left(\begin{array}{c}
n, 6 j+; q \\
6 j+4
\end{array}\right)_{2} \\
-\sum_{j=-\infty}^{\infty} q^{15 j^{2}+11 j+2+n}\left(\begin{array}{c}
n, 6 j+2 ; q \\
6 j+2
\end{array}\right)_{2}+\sum_{j=-\infty}^{\infty} q^{15 j^{2}-11 j+2+n}\left(\begin{array}{c}
n, 6 j-2 ; q \\
6 j-2
\end{array}\right)_{2}
\end{gathered}
$$

In the above, the third and fourth terms cancel after replacing $j$ by $-j$ in the fourth and applying (1.40). To see that the first and second terms cancel, replace $j$ by $1-j$ in the second term, and apply (1.40) to it. 
$=0$.

Now, we shall see that the RHS's of (6.79) and (6.96) satisfy (6.5):

$$
\begin{gathered}
\sum_{j=-\infty}^{\infty} q^{15 j^{2}+j}\left(\begin{array}{c}
n, 6 j ; q \\
6 j
\end{array}\right)_{2}-\sum_{j=-\infty}^{\infty} q^{15 j^{2}+19 j+6}\left(\begin{array}{c}
n, 6 j+4 ; q \\
6 j+4
\end{array}\right)_{2} \\
-\sum_{j=-\infty}^{\infty} q^{15 j^{2}+j}\left(\begin{array}{c}
n-1,6 j ; q \\
6 j
\end{array}\right)_{2}+\sum_{j=-\infty}^{\infty} q^{15 j^{2}+19 j+6}\left(\begin{array}{c}
n-1,6 j+4 ; q \\
6 j+4
\end{array}\right)_{2} \\
-\sum_{j=-\infty}^{\infty} q^{15 j^{2}+j+n-1}\left(\begin{array}{c}
n-2,6 j ; q \\
6 j
\end{array}\right)_{2}+\sum_{j=-\infty}^{\infty} q^{15 j^{2}+19 j+5+n}\left(\begin{array}{c}
n-2,6 j+4 ; q \\
6 j+4
\end{array}\right)_{2} \\
-\sum_{j=-\infty}^{\infty} q^{15 j^{2}+7 j+n}\left(\begin{array}{c}
n-2,6 j+1 ; q \\
6 j+1
\end{array}\right)_{2}+\sum_{j=-\infty}^{\infty} q^{15 j^{2}+17 j+n+4}\left(\begin{array}{c}
n-2,6 j+3 ; q \\
6 j+3
\end{array}\right)_{2}
\end{gathered}
$$

Apply (1.29) to the first term to obtain

$$
\begin{aligned}
& =\sum_{j=-\infty}^{\infty} q^{15 j^{2}+j+n-1}\left(\begin{array}{c}
n-1,6 j ; q \\
6 j
\end{array}\right)_{2}+\sum_{j=-\infty}^{\infty} q^{15 j^{2}-5 j+n}\left(\begin{array}{c}
n-1,6 j-1 ; q \\
6 j-1
\end{array}\right)_{2} \\
& -\sum_{j=-\infty}^{\infty} q^{15 j^{2}+19 j+6}\left(\begin{array}{c}
n, 6 j+4 ; q \\
6 j+4
\end{array}\right)_{2}+\sum_{j=-\infty}^{\infty} q^{15 j^{2}+19 j+6}\left(\begin{array}{c}
n-1,6 j+4 ; q \\
6 j+4
\end{array}\right)_{2} \\
& -\sum_{j=-\infty}^{\infty} q^{15 j^{2}+j+n-1}\left(\begin{array}{c}
n-2,6 j ; q \\
6 j
\end{array}\right)_{2}+\sum_{j=-\infty}^{\infty} q^{15 j^{2}+19 j+5+n}\left(\begin{array}{c}
n-2,6 j+4 ; q \\
6 j+4
\end{array}\right)_{2} \\
& -\sum_{j=-\infty}^{\infty} q^{15 j^{2}+7 j+n}\left(\begin{array}{c}
n-2,6 j+1 ; q \\
6 j+1
\end{array}\right)_{2}+\sum_{j=-\infty}^{\infty} q^{15 j^{2}+17 j+n+4}\left(\begin{array}{c}
n-2,6 j+3 ; q \\
6 j+3
\end{array}\right)_{2}
\end{aligned}
$$

Next, apply (1.27) to the first term, (1.29) to the third term, and apply (1.32) to the seventh term, to wind up with

$$
\begin{aligned}
& =\sum_{j=-\infty}^{\infty} q^{15 j^{2}-5 j+n}\left(\begin{array}{c}
n-1,6 j-1 ; q \\
6 j-1
\end{array}\right)_{2}-\sum_{j=-\infty}^{\infty} q^{15 j^{2}+19 j+n+5}\left(\begin{array}{c}
n-1,6 j+4 ; q \\
6 j+5
\end{array}\right)_{2} \\
& -\sum_{j=-\infty}^{\infty} q^{15 j^{2}+13 j+2+n}\left(\begin{array}{c}
n-1,6 j+3 ; q \\
6 j+3
\end{array}\right)_{2}+\sum_{j=-\infty}^{\infty} q^{15 j^{2}+19 j+5+n}\left(\begin{array}{c}
n-2,6 j+4 ; q \\
6 j+4
\end{array}\right)_{2} \\
& +\sum_{j=-\infty}^{\infty} q^{15 j^{2}+7 j+2 n-2}\left(\begin{array}{c}
n-2,6 j+1 ; q \\
6 j+2
\end{array}\right)_{2}+\sum_{j=-\infty}^{\infty} q^{15 j^{2}+17 j+n+4}\left(\begin{array}{c}
n-2,6 j+3 ; q \\
6 j+3
\end{array}\right)_{2} .
\end{aligned}
$$


Next, apply (1.27) to the second term:

$$
\begin{aligned}
& =\sum_{j=-\infty}^{\infty} q^{15 j^{2}-5 j+n}\left(\begin{array}{c}
n-1,6 j-1 ; q \\
6 j-1
\end{array}\right)_{2}-\sum_{j=-\infty}^{\infty} q^{15 j^{2}+19 j+2 n+3}\left(\begin{array}{c}
n-2,6 j+4 ; q \\
6 j+5
\end{array}\right)_{2} \\
& -\sum_{j=-\infty}^{\infty} q^{15 j^{2}+25 j+10+n}\left(\begin{array}{c}
n-2,6 j+6 ; q \\
6 j+6
\end{array}\right)_{2}-\sum_{j=-\infty}^{\infty} q^{15 j^{2}+13 j+2+n}\left(\begin{array}{c}
n-1,6 j+3 ; q \\
6 j+3
\end{array}\right)_{2} \\
& +\sum_{j=-\infty}^{\infty} q^{15 j^{2}+7 j+2 n-2}\left(\begin{array}{c}
n-2,6 j+1 ; q \\
6 j+2
\end{array}\right)_{2}+\sum_{j=-\infty}^{\infty} q^{15 j^{2}+17 j+n+4}\left(\begin{array}{c}
n-2,6 j+3 ; q \\
6 j+3
\end{array}\right)_{2} .
\end{aligned}
$$

Next, shift $j$ to $j-1$ in the second, third, and fourth terms, apply (1.28) to the first to obtain the following:

$$
\begin{aligned}
& =\sum_{j=-\infty}^{\infty} q^{15 j^{2}-11 j+2 n}\left(\begin{array}{c}
n-2,6 j-2 ; q \\
6 j-2
\end{array}\right)_{2}-\sum_{j=-\infty}^{\infty} q^{15 j^{2}+13 j+n+2}\left(\begin{array}{c}
n-1,6 j+3 ; q \\
6 j+3
\end{array}\right)_{2} \\
& +\sum_{j=-\infty}^{\infty} q^{15 j^{2}+7 j+2 n-2}\left(\begin{array}{c}
n-2,6 j+1 ; q \\
6 j+2
\end{array}\right)_{2}+\sum_{j=-\infty}^{\infty} q^{15 j^{2}-13 j+n+2}\left(\begin{array}{c}
n-2,6 j-3 ; q \\
6 j-3
\end{array}\right)_{2} .
\end{aligned}
$$

Apply (1.30) to the second term:

$$
\begin{aligned}
& =\sum_{j=-\infty}^{\infty} q^{15 j^{2}-11 j+2 n}\left(\begin{array}{c}
n-2,6 j-2 ; q \\
6 j-2
\end{array}\right)_{2}-\sum_{j=-\infty}^{\infty} q^{15 j^{2}+13 j+n+2}\left(\begin{array}{c}
n-2,6 j+3 ; q \\
6 j+3
\end{array}\right)_{2} \\
& -\sum_{j=-\infty}^{\infty} q^{15 j^{2}+19 j+2 n+4}\left(\begin{array}{c}
n-2,6 j+4 ; q \\
6 j+4
\end{array}\right)_{2}+\sum_{j=-\infty}^{\infty} q^{15 j^{2}-13 j+n+2}\left(\begin{array}{c}
n-2,6 j-3 ; q \\
6 j-3
\end{array}\right)_{2} .
\end{aligned}
$$

The first and third terms cancel after shifting $j$ to $j-1$ in the third. The second and fourth terms can be seen to cancel after replacing $j$ by $-j$ in the fourth, and applying (1.40) to the fourth.

$$
=0 \text {. }
$$

Upon checking the initial conditions, the proof is complete.

Identities (6.94) and (6.99) can be proved together in an analogous fashion.

\section{A Annotated Slater List}

Lucy Slater's list of 130 identities of the Rogers-Ramanujan type appeared in [68, p. 152167]. While studying these identities, I found that some identities appeared more than once on the list, usually in slightly different forms. Also, I found misprints in identities 6 , 10, 40, 41, 42, 54, 88, 89, 97, 100, 108, 110, 115, and 128. Some identities are simple sums of others on the list; a few are the sum of an identity on the list with another which does not appear but is easily deduced. Not all of the identities originate with Slater. Some 
date back as far as Euler; many were known to Rogers. I have attempted to provide detailed references for all of the identities which predate Slater.

In all cases, $|q|<1$.

Identity A.1 (Euler, 1748). The Pentagonal Number Theorem. Euler [35, p. 274, sec. 323].

$$
\sum_{n=-\infty}^{\infty}(-1)^{n} q^{3 n(n+1) / 2}=\prod_{n=1}^{\infty}\left(1-q^{n}\right)
$$

Identity A.2 (Euler, 1748). Euler [35]. See also Andrews [6, p. 19, eqn (2.2.6) with $t=q]$. Note: This identity is the same as (7) with $q$ replaced by $\sqrt{q}$.

$$
\sum_{n=0}^{\infty} \frac{q^{n(n+1) / 2}}{(q ; q)_{n}}=\prod_{n=1}^{\infty}\left(1+q^{n}\right)
$$

Identity A.3 (Euler, 1748). Euler [35]. See also Andrews [6, p. 19, eqn (2.2.6) with $q$ replaced by $q^{2}$ and $\left.t=-q\right]$. Note: This identity is the same as (23).

$$
\sum_{n=0}^{\infty} \frac{(-1)^{n} q^{n^{2}}}{\left(q^{2} ; q^{2}\right)_{n}}=\prod_{n=1}^{\infty}\left(1-q^{2 n-1}\right)
$$

Identity A.4.

$$
\sum_{n=0}^{\infty} \frac{(-1)^{n}\left(-q ; q^{2}\right)_{n} q^{n^{2}}}{\left(q^{4} ; q^{4}\right)_{n}}=\prod_{n=1}^{\infty}\left(1-q^{2 n-1}\right)\left(1-q^{4 n-2}\right)
$$

Observation A.5. Note: Identity (5) (with $q$ replaced by $-q$ ) is the same as (9) and (84).

Identity A.6.

$$
\sum_{n=0}^{\infty} \frac{(-1 ; q)_{n} q^{n^{2}}}{(q ; q)_{n}\left(q ; q^{2}\right)_{n}}=\prod_{n=1}^{\infty} \frac{\left(1+q^{3 n-1}\right)\left(1+q^{3 n-2}\right)\left(1-q^{3 n}\right)}{1-q^{n}}
$$

Observation A.7. Identity (7) is the same as (2) with $q$ replaced by $q^{2}$.

Identity A.8 (Gauss-Lebesgue). This is a special case $(z=-q)$ of an identity appearing in Lebesgue [48, pp. 44-47]. See also Andrews [11]. Lebesgue attributes the identity to Gauss [38], however I have been unable to find it there.

$$
\sum_{n=0}^{\infty} \frac{(-q ; q)_{n} q^{n(n+1) / 2}}{(q ; q)_{n}}=\prod_{n=1}^{\infty} \frac{1-q^{4 n}}{1-q^{n}}
$$


Identity A.9 (Jackson, 1928). Jackson [46, p. 179, 3 lines from bottom] Note: Identity (9) is the same as (84) and equivalent to (5) with q replaced by $-q$.

$$
\sum_{n=0}^{\infty} \frac{q^{n(2 n+1)}}{(q ; q)_{2 n+1}}=\prod_{n=1}^{\infty}\left(1+q^{n}\right)
$$

Identity A.10. Note: This identity is the same as (47).

$$
\sum_{n=0}^{\infty} \frac{(-1 ; q)_{2 n} q^{n^{2}}}{\left(q^{2} ; q^{2}\right)_{n}\left(q^{2} ; q^{4}\right)_{n}}=\prod_{n=1}^{\infty}\left(1+q^{2 n-1}\right)\left(1+q^{n}\right)
$$

Identity A.11. Note: This identity is the same as (51) and (64).

$$
\sum_{n=0}^{\infty} \frac{(-q ; q)_{2 n} q^{n(n+1)}}{\left(q ; q^{2}\right)_{n+1}\left(q^{4} ; q^{4}\right)_{n}}=\prod_{n=1}^{\infty}\left(1+q^{n}\right)\left(1+q^{2 n}\right)
$$

Identity A.12 (Gauss-Lebesgue). This is a special case $(z=-1)$ of an identity appearing in Lebesgue [48, pp. 44-47]. Lebesgue attributes the identity to Gauss [38], however I have been unable to find it there. See also Andrews [11].

$$
\sum_{n=0}^{\infty} \frac{(-1 ; q)_{n} q^{n(n+1) / 2}}{(q ; q)_{n}}=\prod_{n=0}^{\infty} \frac{1+q^{2 n+1}}{1-q^{2 n+1}}
$$

Identity A.13.

$$
\sum_{n=0}^{\infty} \frac{(-q ; q)_{n} q^{n(n-1) / 2}}{(q ; q)_{n}}=\prod_{n=1}^{\infty} \frac{1-q^{4 n}}{1-q^{n}}+\prod_{n=1}^{\infty} \frac{1+q^{2 n-1}}{1-q^{2 n-1}}
$$

Identity A.14 (Rogers, 1894). The Second Rogers-Ramanujan Identity. Rogers [57, p. 329 (2)].

$$
\sum_{n=0}^{\infty} \frac{q^{n(n+1)}}{(q ; q)_{n}}=\prod_{n=1}^{\infty} \frac{1}{\left(1-q^{5 n-2}\right)\left(1-q^{5 n-3}\right)}
$$

Identity A.15 (Rogers, 1917). Rogers [58, p. 330 (5)]. This identity appears with a misprint in Jackson [46, p. 170, third eqn.]. Bailey notes and corrects this error in [21, p. 426, eqn 6.3]. It appears that neither Jackson nor Bailey noticed that it had already appeared (correctly) in Rogers [58].

$$
\sum_{n=0}^{\infty} \frac{(-1)^{n} q^{n(3 n-2)}}{\left(-q ; q^{2}\right)_{n}\left(q^{4} ; q^{4}\right)_{n}}=\prod_{n=1}^{\infty} \frac{\left(1-q^{5 n-1}\right)\left(1-q^{5 n-4}\right)\left(1-q^{5 n}\right)}{\left(1-q^{2 n}\right)}
$$

Identity A.16 (Rogers, 1894). Rogers [5\%, p. 331, immediately preceding eqn. (7)].

$$
\sum_{n=0}^{\infty} \frac{q^{n(n+2)}}{\left(q^{4} ; q^{4}\right)_{n}}=\prod_{n=1}^{\infty} \frac{1}{\left(1-q^{5 n-2}\right)\left(1-q^{5 n-3}\right)\left(1+q^{2 n}\right)}
$$


Identity A.17. Note: This identity is equivalent to (A.94) with q replaced by $-q$.

$$
\sum_{n=0}^{\infty} \frac{q^{n(n+1)}}{\left(q^{2} ; q^{2}\right)_{n}\left(-q ; q^{2}\right)_{n+1}}=\prod_{n=1}^{\infty} \frac{\left(1-q^{5 n-1}\right)\left(1-q^{5 n-4}\right)\left(1-q^{5 n}\right)\left(1+q^{2 n}\right)}{\left(1-q^{2 n}\right)}
$$

Identity A.18 (Rogers, 1894). The First Rogers-Ramanujan Identity. Rogers [57, p. 328 (2)].

$$
\sum_{n=0}^{\infty} \frac{q^{n^{2}}}{(q ; q)_{n}}=\prod_{n=1}^{\infty} \frac{1}{\left(1-q^{5 n-1}\right)\left(1-q^{5 n-4}\right)}
$$

Identity A.19 (Rogers, 1894). Rogers [5\%, p. 339, immediately preceeding "Example 3"]

$$
\sum_{n=0}^{\infty} \frac{(-1)^{n} q^{3 n^{2}}}{\left(-q ; q^{2}\right)_{n}\left(q^{4} ; q^{4}\right)_{n}}=\prod_{n=1}^{\infty} \frac{\left(1-q^{5 n-2}\right)\left(1-q^{5 n-3}\right)\left(1-q^{5 n}\right)}{\left(1-q^{2 n}\right)}
$$

Identity A.20 (Rogers, 1894). Rogers [57, p. 330, last eqn.].

$$
\sum_{n=0}^{\infty} \frac{q^{n^{2}}}{\left(q^{4} ; q^{4}\right)_{n}}=\prod_{n=1}^{\infty} \frac{1}{\left(1-q^{5 n-1}\right)\left(1-q^{5 n-4}\right)\left(1+q^{2 n}\right)}
$$

Identity A.21.

$$
\sum_{n=0}^{\infty} \frac{(-1)^{n}\left(q ; q^{2}\right)_{n} q^{n^{2}}}{\left(-q ; q^{2}\right)_{n}\left(q^{4} ; q^{4}\right)_{n}}=\prod_{n=1}^{\infty} \frac{\left(1+q^{5 n-2}\right)\left(1+q^{5 n-3}\right)\left(1-q^{5 n}\right)\left(1-q^{2 n-1}\right)}{\left(1-q^{2 n}\right)}
$$

Identity A.22.

$$
\sum_{n=0}^{\infty} \frac{(-q ; q)_{n} q^{n(n+1)}}{\left(q ; q^{2}\right)_{n+1}(q ; q)_{n}}=\prod_{n=1}^{\infty} \frac{\left(1-q^{6 n-1}\right)\left(1-q^{6 n-5}\right)\left(1-q^{6 n}\right)\left(1+q^{n}\right)}{\left(1-q^{n}\right)}
$$

Observation A.23. Identity (23) is the same as (3).

Identity A.24.

$$
\sum_{n=0}^{\infty} \frac{(-1 ; q)_{2 n} q^{n}}{\left(q^{2} ; q^{2}\right)_{n}}=\prod_{n=1}^{\infty} \frac{\left(1-q^{6 n-3}\right)^{2}\left(1-q^{6 n}\right)\left(1+q^{n}\right)}{\left(1-q^{n}\right)}
$$

Identity A.25.

$$
\sum_{n=0}^{\infty} \frac{\left(-q ; q^{2}\right)_{n} q^{n^{2}}}{\left(q^{4} ; q^{4}\right)_{n}}=\prod_{n=1}^{\infty} \frac{\left(1-q^{6 n-3}\right)^{2}\left(1-q^{6 n}\right)\left(1+q^{2 n-1}\right)}{\left(1-q^{2 n}\right)}
$$


Identity A.26.

$$
\sum_{n=0}^{\infty} \frac{(-q ; q)_{n} q^{n^{2}}}{\left(q ; q^{2}\right)_{n+1}(q ; q)_{n}}=\prod_{n=1}^{\infty} \frac{\left(1-q^{6 n-3}\right)^{2}\left(1-q^{6 n}\right)\left(1+q^{n}\right)}{\left(1-q^{n}\right)}
$$

Identity A.27. Note: This identity is the same as (87).

$$
\sum_{n=0}^{\infty} \frac{\left(-q ; q^{2}\right)_{n} q^{2 n(n+1)}}{\left(q ; q^{2}\right)_{n+1}\left(q^{4} ; q^{4}\right)_{n}}=\prod_{n=1}^{\infty} \frac{\left(1+q^{6 n-1}\right)\left(1+q^{6 n-5}\right)\left(1-q^{6 n}\right)}{\left(1-q^{2 n}\right)}
$$

Identity A.28.

$$
\sum_{n=0}^{\infty} \frac{\left(-q^{2} ; q^{2}\right)_{n} q^{n(n+1)}}{(q ; q)_{2 n+1}}=\prod_{n=1}^{\infty} \frac{\left(1+q^{6 n-1}\right)\left(1+q^{6 n-5}\right)\left(1-q^{6 n}\right)\left(1+q^{2 n}\right)}{\left(1-q^{2 n}\right)}
$$

Identity A.29.

$$
\sum_{n=0}^{\infty} \frac{\left(-q ; q^{2}\right)_{n} q^{n^{2}}}{(q ; q)_{2 n}}=\prod_{n=1}^{\infty} \frac{\left(1+q^{6 n-2}\right)\left(1+q^{6 n-4}\right)\left(1-q^{6 n}\right)\left(1+q^{2 n-1}\right)}{\left(1-q^{2 n}\right)}
$$

Observation A.30. Identity (30) is the same as (24) with $q$ replaced by $-q$.

Identity A.31 (Rogers, 1917). The Third Rogers-Selberg Identity. Rogers [58, p. 331 (6), line 2]. Independently rediscovered by Selberg [66].

$$
\sum_{n=0}^{\infty} \frac{q^{2 n(n+1)}}{\left(q^{2} ; q^{2}\right)_{n}(-q ; q)_{2 n+1}}=\prod_{n=1}^{\infty} \frac{\left(1-q^{7 n-1}\right)\left(1-q^{7 n-6}\right)\left(1-q^{7 n}\right)}{\left(1-q^{2 n}\right)}
$$

Identity A.32 (Rogers, 1894). The Second Rogers-Selberg Identity. Rogers [57, bottom of p. 342]. Independently rediscovered by Selberg [66].

$$
\sum_{n=0}^{\infty} \frac{q^{2 n(n+1)}}{\left(q^{2} ; q^{2}\right)_{n}(-q ; q)_{2 n}}=\prod_{n=1}^{\infty} \frac{\left(1-q^{7 n-2}\right)\left(1-q^{7 n-5}\right)\left(1-q^{7 n}\right)}{\left(1-q^{2 n}\right)}
$$

Identity A.33 (Rogers, 1894). The First Rogers-Selberg Identity. Rogers [57, bottom of p. 339]. Independently rediscovered by Selberg [66].

$$
\sum_{n=0}^{\infty} \frac{q^{2 n^{2}}}{\left(q^{2} ; q^{2}\right)_{n}(-q ; q)_{2 n}}=\prod_{n=1}^{\infty} \frac{\left(1-q^{7 n-3}\right)\left(1-q^{7 n-4}\right)\left(1-q^{7 n}\right)}{\left(1-q^{2 n}\right)}
$$

Identity A.34 (Slater, 1952). The analytic version of the second Göllnitz-Gordon partition identity.

$$
\sum_{n=0}^{\infty} \frac{\left(-q ; q^{2}\right)_{n} q^{n(n+2)}}{\left(q^{2} ; q^{2}\right)_{n}}=\prod_{n=1}^{\infty} \frac{1}{\left(1-q^{8 n-3}\right)\left(1-q^{8 n-4}\right)\left(1-q^{8 n-5}\right)}
$$


Identity A.35. Note: This identity is the same as (106).

$$
\sum_{n=0}^{\infty} \frac{\left(-q ; q^{2}\right)_{n}(-q ; q)_{n} q^{n(n+3) / 2}}{(q ; q)_{2 n+1}}=\prod_{n=1}^{\infty} \frac{\left(1-q^{8 n-1}\right)\left(1-q^{8 n-7}\right)\left(1-q^{8 n}\right)\left(1+q^{n}\right)}{\left(1-q^{n}\right)}
$$

Identity A.36 (Slater, 1952). The analytic version of the first Göllnitz-Gordon partition identity.

$$
\sum_{n=0}^{\infty} \frac{\left(-q ; q^{2}\right)_{n} q^{n^{2}}}{\left(q^{2} ; q^{2}\right)_{n}}=\prod_{n=1}^{\infty} \frac{1}{\left(1-q^{8 n-1}\right)\left(1-q^{8 n-4}\right)\left(1-q^{8 n-7}\right)}
$$

Identity A.37. Note: This identity is the same as (105).

$$
\sum_{n=0}^{\infty} \frac{\left(-q ; q^{2}\right)_{n}(-q ; q)_{n} q^{n(n+1) / 2}}{(q ; q)_{2 n+1}}=\prod_{n=1}^{\infty} \frac{\left(1-q^{8 n-3}\right)\left(1-q^{8 n-5}\right)\left(1-q^{8 n}\right)\left(1+q^{n}\right)}{\left(1-q^{n}\right)}
$$

Identity A.38. Note: This identity is the same as (86).

$$
\sum_{n=0}^{\infty} \frac{q^{2 n(n+1)}}{(q ; q)_{2 n+1}}=\prod_{n=1}^{\infty} \frac{\left(1-q^{8 n-3}\right)\left(1-q^{8 n-5}\right)\left(1-q^{8 n}\right)\left(1-q^{16 n-2}\right)\left(1-q^{16 n-14}\right)}{\left(1-q^{n}\right)}
$$

Identity A.39 (Jackson, 1928). Note: This identity is the same as (83). Jackson [46, p. 170, 5th eqn.].

$$
\sum_{n=0}^{\infty} \frac{q^{2 n^{2}}}{(q ; q)_{2 n}}=\prod_{n=1}^{\infty} \frac{\left(1-q^{8 n-1}\right)\left(1-q^{8 n-7}\right)\left(1-q^{8 n}\right)\left(1-q^{16 n-6}\right)\left(1-q^{16 n-10}\right)}{\left(1-q^{n}\right)}
$$

Identity A.40 (Bailey, 1947). Bailey [21, p. 422, eqn. (1.7)].

$$
\sum_{n=0}^{\infty} \frac{(q ; q)_{3 n+1} q^{3 n(n+1)}}{\left(q^{3} ; q^{3}\right)_{n}\left(q^{3} ; q^{3}\right)_{2 n+1}}=\prod_{n=1}^{\infty} \frac{\left(1-q^{9 n-1}\right)\left(1-q^{9 n-8}\right)\left(1-q^{9 n}\right)}{\left(1-q^{3 n}\right)}
$$

Identity A.41 (Bailey, 1947). Bailey [21, p. 422, eqn. (1.8)].

$$
\sum_{n=0}^{\infty} \frac{(q ; q)_{3 n}\left(1-q^{3 n+2}\right) q^{3 n(n+1)}}{\left(q^{3} ; q^{3}\right)_{n}\left(q^{3} ; q^{3}\right)_{2 n+1}}=\prod_{n=1}^{\infty} \frac{\left(1-q^{9 n-2}\right)\left(1-q^{9 n-7}\right)\left(1-q^{9 n}\right)}{\left(1-q^{3 n}\right)}
$$

Identity A.42 (Bailey, 1947). Bailey [21, p. 422, eqn. (1.6)].

$$
\sum_{n=0}^{\infty} \frac{(q ; q)_{3 n} q^{3 n^{2}}}{\left(q^{3} ; q^{3}\right)_{n}\left(q^{3} ; q^{3}\right)_{2 n}}=\prod_{n=1}^{\infty} \frac{\left(1-q^{9 n-4}\right)\left(1-q^{9 n-5}\right)\left(1-q^{9 n}\right)}{\left(1-q^{3 n}\right)}
$$




\section{Identity A.43.}

$$
\sum_{n=0}^{\infty} \frac{(-q ; q)_{n} q^{n(n+3) / 2}}{\left(q ; q^{2}\right)_{n+1}(q ; q)_{n}}=\prod_{n=1}^{\infty} \frac{\left(1-q^{10 n-1}\right)\left(1-q^{10 n-9}\right)\left(1-q^{10 n}\right)\left(1+q^{n}\right)}{\left(1-q^{n}\right)}
$$

Identity A.44 (Rogers, 1917). Rogers [58, p. 330 (2), line 2] Note: this identity is the same as (63).

$$
\sum_{n=0}^{\infty} \frac{q^{3 n(n+1) / 2}}{\left(q ; q^{2}\right)_{n+1}(q ; q)_{n}}=\prod_{n=1}^{\infty} \frac{\left(1-q^{10 n-2}\right)\left(1-q^{10 n-8}\right)\left(1-q^{10 n}\right)}{\left(1-q^{n}\right)}
$$

Identity A.45.

$$
\sum_{n=0}^{\infty} \frac{(-q ; q)_{n} q^{n(n+1) / 2}}{\left(q ; q^{2}\right)_{n+1}(q ; q)_{n}}=\prod_{n=1}^{\infty} \frac{\left(1-q^{10 n-3}\right)\left(1-q^{10 n-7}\right)\left(1-q^{10 n}\right)\left(1+q^{n}\right)}{\left(1-q^{n}\right)}
$$

Identity A.46 (Rogers, 1917). Rogers [58, p. 330 (2) line 3]. This identity is equivalent to (62): See Andrews [9, p. 20, eqn. (8.7)]

$$
\sum_{n=0}^{\infty} \frac{q^{n(3 n-1) / 2}}{\left(q ; q^{2}\right)_{n}(q ; q)_{n}}=\prod_{n=1}^{\infty} \frac{\left(1-q^{10 n-4}\right)\left(1-q^{10 n-6}\right)\left(1-q^{10 n}\right)}{\left(1-q^{n}\right)}
$$

Identity A.47. Note: This identity equivalent to (10), and to (54) $+\times \times(49)$.

$$
\sum_{n=0}^{\infty} \frac{\left(-1 ; q^{2}\right)_{n} q^{n^{2}}}{(q ; q)_{2 n}}=\prod_{n=1}^{\infty} \frac{1+q^{2 n-1}}{1-q^{2 n-1}}
$$

Identity A.48. Note: This identity is (54) $-q \times(49)$.

$$
\begin{gathered}
\sum_{n=0}^{\infty} \frac{\left(-1 ; q^{2}\right)_{n} q^{n(n+1)}}{(q ; q)_{2 n}} \\
=\prod_{n=1}^{\infty} \frac{\left(1-q^{12 n-5}\right)\left(1-q^{12 n-7}\right)\left(1-q^{12 n}\right)}{1-q^{n}}-q \frac{\left(1-q^{12 n-1}\right)\left(1-q^{12 n-11}\right)\left(1-q^{12 n}\right)}{1-q^{n}}
\end{gathered}
$$

Identity A.49.

$$
\sum_{n=0}^{\infty} \frac{\left(-q^{2} ; q^{2}\right)_{n}\left(1-q^{n+1}\right) q^{n(n+2)}}{(q ; q)_{2 n+2}}=\prod_{n=1}^{\infty} \frac{\left(1-q^{12 n-1}\right)\left(1-q^{12 n-11}\right)\left(1-q^{12 n}\right)}{\left(1-q^{n}\right)}
$$

Identity A.50.

$$
\sum_{n=0}^{\infty} \frac{\left(-q ; q^{2}\right)_{n} q^{n(n+2)}}{(q ; q)_{2 n+1}}=\prod_{n=1}^{\infty} \frac{\left(1-q^{12 n-2}\right)\left(1-q^{12 n-10}\right)\left(1-q^{12 n}\right)}{\left(1-q^{n}\right)}
$$


Observation A.51. Identity (51) is the same as (11) and (64).

Identity A.52. Note: This identity is the same as (85).

$$
\sum_{n=0}^{\infty} \frac{q^{n(2 n-1)}}{(q ; q)_{2 n}}=\prod_{n=1}^{\infty}\left(1+q^{n}\right)
$$

Identity A.53.

$$
\sum_{n=0}^{\infty} \frac{\left(q ; q^{2}\right)_{2 n} q^{4 n^{2}}}{\left(q^{4} ; q^{4}\right)_{2 n}}=\prod_{n=1}^{\infty} \frac{\left(1-q^{12 n-5}\right)\left(1-q^{12 n-7}\right)\left(1-q^{12 n}\right)}{\left(1-q^{4 n}\right)}
$$

Identity A.54.

$$
\sum_{n=0}^{\infty} \frac{\left(-q^{2} ; q^{2}\right)_{n-1}\left(1+q^{n}\right) q^{n^{2}}}{(q ; q)_{2 n}}=\prod_{n=1}^{\infty} \frac{\left(1-q^{12 n-5}\right)\left(1-q^{12 n-7}\right)\left(1-q^{12 n}\right)}{\left(1-q^{n}\right)}
$$

Identity A.55.

$$
\sum_{n=0}^{\infty} \frac{\left(q ; q^{2}\right)_{2 n+1} q^{4 n(n+1)}}{\left(q^{4} ; q^{4}\right)_{2 n+1}}=\prod_{n=1}^{\infty} \frac{\left(1-q^{12 n-1}\right)\left(1-q^{12 n-11}\right)\left(1-q^{12 n}\right)}{\left(1-q^{4 n}\right)}
$$

Identity A.56.

$$
\sum_{n=0}^{\infty} \frac{(-q ; q)_{n} q^{n(n+2)}}{\left(q ; q^{2}\right)_{n+1}(q ; q)_{n+1}}=\prod_{n=1}^{\infty} \frac{\left(1+q^{12 n-1}\right)\left(1+q^{12 n-11}\right)\left(1-q^{12 n}\right)}{\left(1-q^{n}\right)}
$$

Identity A.57. Note: This identity is the same as (55) with q replaced by $-q$.

$$
\sum_{n=0}^{\infty} \frac{\left(-q ; q^{2}\right)_{2 n+1} q^{4 n(n+1)}}{\left(q^{4} ; q^{4}\right)_{2 n+1}}=\prod_{n=1}^{\infty} \frac{\left(1+q^{12 n-1}\right)\left(1+q^{12 n-11}\right)\left(1-q^{12 n}\right)}{\left(1-q^{4 n}\right)}
$$

Identity A.58.

$$
1+\sum_{n=1}^{\infty} \frac{(-q ; q)_{n-1} q^{n^{2}}}{\left(q ; q^{2}\right)_{n}(q ; q)_{n}}=\prod_{n=1}^{\infty} \frac{\left(1+q^{12 n-5}\right)\left(1+q^{12 n-7}\right)\left(1-q^{12 n}\right)}{\left(1-q^{n}\right)}
$$

Identity A.59 (Rogers, 1917). Rogers [58, p. 329 (1), line 3]

$$
\sum_{n=0}^{\infty} \frac{q^{n(n+2)}}{\left(q ; q^{2}\right)_{n+1}(q ; q)_{n}}=\prod_{n=1}^{\infty} \frac{\left(1-q^{14 n-2}\right)\left(1-q^{14 n-12}\right)\left(1-q^{14 n}\right)}{\left(1-q^{n}\right)}
$$

Identity A.60 (Rogers, 1917). Rogers [58, p. 329 (1), line 2]

$$
\sum_{n=0}^{\infty} \frac{q^{n(n+1)}}{\left(q ; q^{2}\right)_{n+1}(q ; q)_{n}}=\prod_{n=1}^{\infty} \frac{\left(1-q^{14 n-4}\right)\left(1-q^{14 n-10}\right)\left(1-q^{14 n}\right)}{\left(1-q^{n}\right)}
$$


Identity A.61 (Rogers, 1894). Rogers [57, p. 341, ex. 2]

$$
\sum_{n=0}^{\infty} \frac{q^{n^{2}}}{\left(q ; q^{2}\right)_{n}(q ; q)_{n}}=\prod_{n=1}^{\infty} \frac{\left(1-q^{14 n-6}\right)\left(1-q^{14 n-8}\right)\left(1-q^{14 n}\right)}{\left(1-q^{n}\right)}
$$

Identity A.62. This identity is equivalent to (46): See Andrews [9, p. 20, eqn. (8.7)].

$$
\sum_{n=0}^{\infty} \frac{(-q ; q)_{n} q^{n(3 n+1) / 2}}{(q ; q)_{2 n+1}}=\prod_{n=1}^{\infty} \frac{\left(1-q^{10 n-4}\right)\left(1-q^{10 n-6}\right)\left(1-q^{10 n}\right)}{\left(1-q^{n}\right)}
$$

Observation A.63. Identity (63) is the same as (44).

Observation A.64. Identity (64) is the same as (11) and (51).

Observation A.65. Note: Identity (65) is $(37)+\sqrt{q} \times(35)$, with $q$ replaced by $q^{2}$ throughout.

Identity A.66. Note: Identity (66) is (71) $+q \times(68)$

$$
\sum_{n=0}^{\infty} \frac{\left(-1 ; q^{4}\right)_{n}\left(-q ; q^{2}\right)_{n} q^{n^{2}}}{\left(q^{2} ; q^{2}\right)_{2 n}}=\frac{\left(q^{6}, q^{10}, q^{16} ; q^{16}\right)_{\infty}+q\left(q^{2}, q^{14}, q^{16} ; q^{16}\right)_{\infty}}{\left(q, q^{2}\right)_{\infty}\left(q^{4} ; q^{4}\right)_{\infty}}
$$

Identity A.67. Note: Identity (67) is (71) $-q \times(68)$

$$
\sum_{n=0}^{\infty} \frac{\left(-1 ; q^{4}\right)_{n}\left(-q ; q^{2}\right)_{n} q^{n(n+2)}}{\left(q^{2} ; q^{2}\right)_{2 n}}=\frac{\left(q^{6}, q^{10}, q^{16} ; q^{16}\right)_{\infty}-q\left(q^{2}, q^{14}, q^{16} ; q^{16}\right)_{\infty}}{\left(q, q^{2}\right)_{\infty}\left(q^{4} ; q^{4}\right)_{\infty}}
$$

\section{Identity A.68.}

$$
\sum_{n=0}^{\infty} \frac{\left(-q^{;} q^{2}\right)_{n}\left(-q^{4} ; q^{4}\right)_{n} q^{n(n+2)}}{\left(-q^{2} ; q^{2}\right)_{n+1}\left(q^{2} ; q^{4}\right)_{n+1}\left(q^{2} ; q^{2}\right)_{n}}=\prod_{n=1}^{\infty} \frac{\left(1-q^{16 n-2}\right)\left(1-q^{16 n-14}\right)\left(1-q^{16 n}\right)\left(1+q^{2 n-1}\right)}{\left(1-q^{2 n}\right)}
$$

Identity A.69.

$$
\sum_{n=0}^{\infty} \frac{\left(-q^{2} ; q^{2}\right)_{n} q^{n(n+2)}}{(q ; q)_{2 n+2}}=\prod_{n=1}^{\infty}\left[\left(1+q^{16 n-2}\right)\left(1+q^{16 n-14}\right)\left(1-q^{16 n}\right)\right] \frac{\left(1+q^{2 n-1}\right)}{\left(1-q^{2 n}\right)}
$$

Identity A.70.

$$
\sum_{n=0}^{\infty} \frac{\left(-q ; q^{2}\right)_{n+1}\left(-q^{2} ; q^{4}\right)_{n} q^{n(n+2)}}{\left(q^{2} ; q^{4}\right)_{n+1}\left(q^{4} ; q^{4}\right)_{n}}=\prod_{n=1}^{\infty} \frac{\left(1-q^{16 n-4}\right)\left(1-q^{16 n-12}\right)\left(1-q^{16 n}\right)\left(1+q^{2 n-1}\right)}{\left(1-q^{2 n}\right)}
$$




\section{Identity A.71.}

$$
\sum_{n=0}^{\infty} \frac{\left(-q^{4} ; q^{4}\right)_{n-1}\left(-q ; q^{2}\right)_{n} q^{n^{2}}}{\left(q^{2} ; q^{4}\right)_{n}\left(q^{2} ; q^{2}\right)_{n}\left(-q^{2} ; q^{2}\right)_{n-1}}=\prod_{n=1}^{\infty} \frac{\left(1-q^{16 n-6}\right)\left(1-q^{16 n-10}\right)\left(1-q^{16 n}\right)\left(1+q^{2 n-1}\right)}{\left(1-q^{2 n}\right)}
$$

\section{Identity A.72.}

$$
1+\sum_{n=1}^{\infty} \frac{\left(-q^{4} ; q^{4}\right)_{n-1}\left(-q ; q^{2}\right)_{n} q^{n^{2}}}{\left(q^{2} ; q^{4}\right)_{n}\left(q^{2} ; q^{2}\right)_{n}\left(q^{2} ; q^{2}\right)_{n-1}}=\prod_{n=1}^{\infty} \frac{\left(1-q^{16 n+6}\right)\left(1-q^{16 n+10}\right)\left(1-q^{16 n}\right)\left(1+q^{2 n-1}\right)}{\left(1-q^{2 n}\right)}
$$

Identity 72-a. Note: This identity does not appear explictly in Slater's list. It appears implicitly as the sum of (130) and $q \times(70)$.

$$
\sum_{n=0}^{\infty} \frac{\left(-q^{4} ; q^{4}\right)_{n}\left(-q ; q^{2}\right)_{n+1}\left(1+q^{2 n+1}\right) q^{n^{2}}}{\left(q^{2} ; q^{2}\right)_{2 n+2}}=\prod_{n=1}^{\infty} \frac{\left(1-q^{16 n-8}\right)^{2}\left(1-q^{16 n}\right)\left(1+q^{2 n-1}\right)}{1-q^{2 n}}
$$

Identity A.73. Note: Identity (73) is the sum of (77) and (78).

$$
1+\sum_{n=1}^{\infty} \frac{\left(q^{3} ; q^{3}\right)_{n-1}(-q ; q)_{n} q^{n(n-1) / 2}}{(q ; q)_{2 n-1}(q ; q)_{n}}=\frac{\left(q^{6}, q^{12}, q^{18} ; q^{18}\right)_{\infty}+\left(q^{9}, q^{9}, q^{18} ; q^{18}\right)_{\infty}}{(q ; q)_{\infty}\left(q ; q^{2}\right)_{\infty}}
$$

Identity A.74. Note: Identity (74) is $(77)+(78)-q \times(76)$.

$$
\begin{gathered}
1+\sum_{n=1}^{\infty} \frac{\left(q^{3} ; q^{3}\right)_{n-1}(-q ; q)_{n} q^{n(n-1) / 2}}{(q ; q)_{2 n}(q ; q)_{n-1}} \\
=\frac{\left(q^{6}, q^{12}, q^{18} ; q^{18}\right)_{\infty}+\left(q^{9}, q^{9}, q^{18} ; q^{18}\right)_{\infty}-q\left(q^{3}, q^{15}, q^{18} ; q^{18}\right)_{\infty}}{(q ; q)_{\infty}\left(q ; q^{2}\right)_{\infty}}
\end{gathered}
$$

Observation A.75. Note: Identity (75) is (78) $-q \times(76)$.

$$
1+\sum_{n=1}^{\infty} \frac{\left(q^{3} ; q^{3}\right)_{n-1}(-q ; q)_{n} q^{n(n+1) / 2}}{(q ; q)_{2 n}(q ; q)_{n-1}}=\frac{\left(q^{9}, q^{9}, q^{18} ; q^{18}\right)_{\infty}-q\left(q^{3}, q^{15}, q^{18} ; q^{18}\right)_{\infty}}{(q ; q)_{\infty}\left(q ; q^{2}\right)_{\infty}}
$$

Identity A.76 (Bailey-Dyson, 1947). Appears in Bailey [21, p. 434, (D1)]; Bailey credits its derivation to Dyson.

$$
\sum_{n=0}^{\infty} \frac{\left(q^{3} ; q^{3}\right)_{n}(-q ; q)_{n+1} q^{n(n+3) / 2}}{(q ; q)_{2 n+2}(q ; q)_{n}}=\prod_{n=1}^{\infty} \frac{\left(1-q^{18 n-3}\right)\left(1-q^{18 n-15}\right)\left(1-q^{18 n}\right)\left(1+q^{n}\right)}{\left(1-q^{n}\right)}
$$


Identity A.77.

$\sum_{n=0}^{\infty} \frac{\left(q^{3} ; q^{3}\right)_{n}(-q ; q)_{n+1}\left(1-q^{n+1}\right) q^{n(n+1) / 2}}{(q ; q)_{2 n+2}(q ; q)_{n}}=\prod_{n=1}^{\infty} \frac{\left(1-q^{18 n-6}\right)\left(1-q^{18 n-12}\right)\left(1-q^{18 n}\right)\left(1+q^{n}\right)}{\left(1-q^{n}\right)}$

Identity A.78 (Bailey-Dyson, 1947). Note: This identity is $(75)+q \times(76)$. Appears in Bailey [21, p. 434, (D3)]; Bailey credits its derivation to Dyson.

$$
1+\sum_{n=1}^{\infty} \frac{\left(q^{3} ; q^{3}\right)_{n-1}(-1 ; q)_{n+1} q^{n(n+1) / 2}}{(q ; q)_{2 n}(q ; q)_{n-1}}=\prod_{n=1}^{\infty} \frac{\left(1-q^{18 n-9}\right)^{2}\left(1-q^{18 n}\right)\left(1+q^{n}\right)}{\left(1-q^{n}\right)}
$$

Identity A.79 (Rogers, 1894). Rogers [5\%, p. 330 second eqn. from top]. Note: This identity is the same as (98).

$$
\sum_{n=0}^{\infty} \frac{q^{n^{2}}}{(q ; q)_{2 n}}=\prod_{n=1}^{\infty} \frac{\left(1-q^{20 n-8}\right)\left(1-q^{20 n-12}\right)\left(1-q^{20 n}\right)\left(1+q^{2 n-1}\right)}{\left(1-q^{2 n}\right)}
$$

Identity A.80 (Rogers, 1917). Rogers [58, p. 331 (1), line 2].

$$
\begin{gathered}
\sum_{n=0}^{\infty} \frac{q^{n(n+1) / 2}}{\left(q ; q^{2}\right)_{n+1}(q ; q)_{n}} \\
=\prod_{n=1}^{\infty}\left[\left(1-q^{7 n-2}\right)\left(1-q^{7 n-5}\right)\left(1-q^{7 n}\right)\left(1-q^{14 n-3}\right)\left(1-q^{14 n-11}\right)\right] \frac{\left(1+q^{n}\right)}{\left(1-q^{n}\right)}
\end{gathered}
$$

Identity A.81 (Rogers, 1917). Rogers [58, p. 331 (1), line 1].

$$
\begin{gathered}
\sum_{n=0}^{\infty} \frac{q^{n(n+1) / 2}}{\left(q ; q^{2}\right)_{n}(q ; q)_{n}} \\
=\prod_{n=1}^{\infty}\left[\left(1-q^{7 n-1}\right)\left(1-q^{7 n-6}\right)\left(1-q^{7 n}\right)\left(1-q^{14 n-5}\right)\left(1-q^{14 n-9}\right)\right] \frac{\left(1+q^{n}\right)}{\left(1-q^{n}\right)}
\end{gathered}
$$

Identity A.82 (Rogers, 1917). Rogers [58, p. 331 (1), line 3].

$$
\begin{gathered}
\sum_{n=0}^{\infty} \frac{q^{n(n+3) / 2}}{\left(q ; q^{2}\right)_{n+1}(q ; q)_{n}} \\
=\prod_{n=1}^{\infty}\left[\left(1-q^{7 n-3}\right)\left(1-q^{7 n-4}\right)\left(1-q^{7 n}\right)\left(1-q^{14 n-1}\right)\left(1-q^{14 n-13}\right)\right] \frac{\left(1+q^{n}\right)}{\left(1-q^{n}\right)}
\end{gathered}
$$

Observation A.83. Identity (83) is the same as (39).

Observation A.84. Identity (84) is the same as (9). 
Observation A.85. Identity (85) is the same as (52).

Observation A.86. Identity (86) is the same as (38).

Observation A.87. Identity (87) is the same as (27).

Identity A.88. Note: This identity is (91) $-q^{2} \times(90)$.

$$
\sum_{n=1}^{\infty} \frac{\left(q^{3} ; q^{3}\right)_{n-1}\left(1-q^{n+1}\right) q^{n^{2}-1}}{(q ; q)_{2 n}(q ; q)_{n-1}}=\frac{\left(q^{6}, q^{21}, q^{27} ; q^{27}\right)_{\infty}-q^{2}\left(q^{3}, q^{24}, q^{27} ; q^{27}\right)_{\infty}}{(q ; q)_{\infty}}
$$

Identity A.89. Note: This identity is (93) $-q \times(91)$.

$$
1+\sum_{n=1}^{\infty} \frac{\left(q^{3} ; q^{3}\right)_{n-1} q^{n(n+1)}}{(q ; q)_{2 n}(q ; q)_{n-1}}=\frac{\left(q^{12}, q^{15}, q^{27} ; q^{27}\right)_{\infty}-q\left(q^{6}, q^{21}, q^{27} ; q^{27}\right)_{\infty}}{(q ; q)_{\infty}}
$$

Identity A.90 (Bailey-Dyson, 1947). Appears in Bailey [21, p. 434, (B1)]; Bailey credits its derivation to Dyson.

$$
\sum_{n=0}^{\infty} \frac{\left(q^{3} ; q^{3}\right)_{n} q^{n(n+3)}}{(q ; q)_{2 n+2}(q ; q)_{n}}=\prod_{n=1}^{\infty} \frac{\left(1-q^{27 n-3}\right)\left(1-q^{27 n-24}\right)\left(1-q^{27 n}\right)}{1-q^{n}}
$$

Identity A.91 (Bailey-Dyson, 1947). Appears in Bailey [21, p. 434, (B2)]; Bailey credits its derivation to Dyson.

$$
\sum_{n=0}^{\infty} \frac{\left(q^{3} ; q^{3}\right)_{n} q^{n(n+2)}}{(q ; q)_{2 n+2}(q ; q)_{n}}=\prod_{n=1}^{\infty} \frac{\left(1-q^{27 n-6}\right)\left(1-q^{27 n-18}\right)\left(1-q^{27 n}\right)}{1-q^{n}}
$$

Identity A.92 (Bailey-Dyson, 1947). Appears in Bailey [21, p. 434, (B3)]; Bailey credits its derivation to Dyson.

$$
\sum_{n=0}^{\infty} \frac{\left(q^{3} ; q^{3}\right)_{n} q^{n(n+1)}}{(q ; q)_{2 n+1}(q ; q)_{n}}=\prod_{n=1}^{\infty} \frac{1-q^{9 n}}{1-q^{n}}
$$

Identity A.93 (Bailey-Dyson, 1947). Appears in Bailey [21, p. 434, (B4)]; Bailey credits its derivation to Dyson.

$$
1+\sum_{n=1}^{\infty} \frac{\left(q^{3} ; q^{3}\right)_{n-1} q^{n^{2}}}{(q ; q)_{2 n-1}(q ; q)_{n}}=\prod_{n=1}^{\infty} \frac{\left(1-q^{27 n-12}\right)\left(1-q^{27 n-15}\right)\left(1-q^{27 n}\right)}{1-q^{n}}
$$

Identity A.94 (Rogers, 1894). Equivalent to Rogers [57, p. 331, eqn. (6)].

$$
\sum_{n=0}^{\infty} \frac{q^{n(n+1)}}{(q ; q)_{2 n+1}}=\prod_{n=1}^{\infty} \frac{\left(1-q^{10 n-3}\right)\left(1-q^{10 n-7}\right)\left(1-q^{10 n}\right)\left(1-q^{20 n-4}\right)\left(1-q^{20 n-16}\right)}{1-q^{n}}
$$


Identity A.95. Note: This identity is equivalent to (97).

$$
\begin{gathered}
\sum_{n=0}^{\infty} \frac{\left(-q ; q^{2}\right)_{n} q^{n(3 n-2)}}{\left(q^{2} ; q^{2}\right)_{2 n}} \\
=\prod_{n=1}^{\infty}\left(1-q^{10 n-3}\right)\left(1-q^{10 n-7}\right)\left(1-q^{10 n}\right)\left(1-q^{20 n-4}\right)\left(1-q^{20 n-16}\right) \cdot \frac{1+q^{2 n-1}}{1-q^{2 n}}
\end{gathered}
$$

Identity A.96 (Rogers, 1894). Equivalent to Rogers [57, p. 331, eqn. (7)].

$$
\sum_{n=0}^{\infty} \frac{q^{n(n+2)}}{(q ; q)_{2 n+1}}=\prod_{n=1}^{\infty} \frac{\left(1-q^{10 n-4}\right)\left(1-q^{10 n-6}\right)\left(1-q^{10 n}\right)\left(1-q^{20 n-2}\right)\left(1-q^{20 n-18}\right)}{1-q^{n}}
$$

Identity A.97. Note: This identity is equivalent to (95).

$$
\begin{gathered}
\sum_{n=0}^{\infty} \frac{\left(-q ; q^{2}\right)_{n+1} q^{n(3 n+2)}}{\left(q^{2} ; q^{2}\right)_{2 n+1}} \\
=\prod_{n=1}^{\infty}\left(1-q^{10 n-3}\right)\left(1-q^{10 n-7}\right)\left(1-q^{10 n}\right)\left(1-q^{20 n-4}\right)\left(1-q^{20 n-16}\right) \cdot \frac{1+q^{2 n-1}}{1-q^{2 n}}
\end{gathered}
$$

Observation A.98. Identity (98) is the same as (79).

Identity A.99 (Rogers, 1894). Equivalent to Rogers [5\%, p. 332, between eqns. (12) and (13)].

$$
\sum_{n=0}^{\infty} \frac{q^{n(n+1)}}{(q ; q)_{2 n}}=\prod_{n=1}^{\infty} \frac{\left(1-q^{10 n-1}\right)\left(1-q^{10 n-9}\right)\left(1-q^{10 n}\right)\left(1-q^{20 n-8}\right)\left(1-q^{20 n-12}\right)}{1-q^{n}}
$$

Identity A.100.

$$
\begin{gathered}
\sum_{n=0}^{\infty} \frac{\left(-q ; q^{2}\right)_{n} q^{3 n^{2}}}{\left(q^{2} ; q^{2}\right)_{2 n}} \\
=\prod_{n=1}^{\infty}\left(1-q^{10 n-1}\right)\left(1-q^{10 n-9}\right)\left(1-q^{10 n}\right)\left(1-q^{20 n-8}\right)\left(1-q^{20 n-12}\right) \cdot \frac{1+q^{2 n-1}}{1-q^{2 n}}
\end{gathered}
$$

Identity A.101. Note: This identity is the sum of (104) and (105-a).

$$
\begin{gathered}
1+\sum_{n=1}^{\infty} \frac{(-q ; q)_{n}\left(-q^{2} ; q^{2}\right)_{n-1} q^{n(n-1) / 2}}{(q ; q)_{2 n}} \\
=\frac{\left(q^{2}, q^{6}, q^{8} ; q^{8}\right)_{\infty}+\left(-q^{16},-q^{16}, q^{32} ; q^{32}\right)_{\infty}-q\left(-q^{8},-q^{24}, q^{32} ; q^{32}\right)_{\infty}}{(q ; q)_{\infty}\left(q ; q^{2}\right)_{\infty}}
\end{gathered}
$$


Identity A.102. Note: This identity is $(105-a)+q \times(103)$

$$
\begin{gathered}
\sum_{n=0}^{\infty} \frac{(-q ; q)_{n+1}\left(-q^{2} ; q^{2}\right)_{n} q^{n(n+1) / 2}}{(q, q)_{2 n+2}} \\
=\frac{\left(-q^{12},-q^{20}, q^{32} ; q^{32}\right)_{\infty}-q^{2}\left(-q^{4},-q^{28}, q^{32} ; q^{32}\right)_{\infty}+q\left(-q^{8},-q^{24}, q^{32} ; q^{32}\right)_{\infty}-q^{4}\left(-1,-q^{32}, q^{32} ; q^{32}\right)_{\infty}}{(q ; q)_{\infty}\left(q ; q^{2}\right)_{\infty}}
\end{gathered}
$$

Identity A.103.

$$
\sum_{n=0}^{\infty} \frac{(-q ; q)_{n+1}\left(-q^{2} ; q^{2}\right)_{n} q^{n(n+3) / 2}}{(q ; q)_{2 n+2}}=\frac{\left(-q^{8},-q^{24}, q^{32} ; q^{32}\right)_{\infty}-q^{3}\left(-1,-q^{32}, q^{32} ; q^{32}\right)_{\infty}}{(q ; q)_{\infty}\left(q ; q^{2}\right)_{\infty}}
$$

\section{Identity A.104.}

$$
1+\sum_{n=1}^{\infty} \frac{(-q ; q)_{n}\left(-q^{2} ; q^{2}\right)_{n-1} q^{n(n+1) / 2}}{(q ; q)_{2 n}}=\frac{\left(-q^{16},-q^{16}, q^{32} ; q^{32}\right)_{\infty}-q\left(-q^{8},-q^{24}, q^{32} ; q^{32}\right)_{\infty}}{(q ; q)_{\infty}\left(q ; q^{2}\right)_{\infty}}
$$

Observation A.105. Identity (105) is the same as (37).

Identity A.105-a. This identity does not appear explicitly in Slater's list. It appears implicilty as the difference of (101) and (104), and as the difference of (102) and $q \times(103)$.

$$
\sum_{n=0}^{\infty} \frac{\left(-q^{2} ; q^{2}\right)_{n} q^{n(n+1) / 2}}{\left(q ; q^{2}\right)_{n+1}(q ; q)_{n}}=\prod_{n=1}^{\infty} \frac{\left(1-q^{8 n-2}\right)\left(1-q^{8 n-6}\right)\left(1-q^{8 n}\right)\left(1+q^{n}\right)}{1-q^{n}}
$$

Observation A.106. Identity (106) is the same as (35).

Identity A.107.

$$
\begin{gathered}
\sum_{n=0}^{\infty} \frac{\left(q^{3} ; q^{6}\right)_{n}\left(-q^{2} ; q^{2}\right)_{n} q^{n(n+1)}}{\left(q^{2} ; q^{2}\right)_{2 n+1}\left(q ; q^{2}\right)_{n}} \\
=\prod_{n=1}^{\infty} \frac{\left(1+q^{12 n-3}\right)\left(1+q^{12 n-9}\right)\left(1-q^{12 n}\right)\left(1-q^{24 n-6}\right)\left(1-q^{24 n-18}\right)\left(1+q^{2 n}\right)}{1-q^{2 n}}
\end{gathered}
$$

Identity A.108. Note: This identity is $(115)-q^{2} \times(116)$.

$$
\begin{gathered}
\sum_{n=0}^{\infty} \frac{\left(q^{6} ; q^{6}\right)_{n}\left(-q ; q^{2}\right)_{n+1}\left(1-q^{2 n+2}\right) q^{n(n+2)}}{\left(q^{2} ; q^{2}\right)_{2 n+2}\left(q^{2} ; q^{2}\right)_{n}} \\
=\prod_{n=1}^{\infty}\left[\left(1+q^{12 n-5}\right)\left(1+q^{12 n-7}\right)\left(1-q^{12 n}\right)\left(1-q^{24 n-2}\right)\left(1-q^{24 n-22}\right)\right] \frac{\left(1+q^{2 n-1}\right)}{\left(1-q^{2 n}\right)}
\end{gathered}
$$




\section{Identity A.109.}

$$
\sum_{n=0}^{\infty} \frac{\left(q^{3} ; q^{6}\right)_{n}\left(-q ; q^{2}\right)_{n+1} q^{n^{2}}}{\left(q^{2} ; q^{2}\right)_{2 n+1}\left(q ; q^{2}\right)_{n}}=\frac{\left(-q^{2},-q^{10}, q^{12} ; q^{12}\right)_{\infty}\left(q^{8}, q^{16} ; q^{24}\right)_{\infty}+q\left(q^{12} ; q^{12}\right)_{\infty}}{\left(q^{4} ; q^{4}\right)_{\infty}\left(q ; q^{2}\right)_{\infty}}
$$

Identity A.109-a. This identity does not appear explicitly in Slater's list. It appears implicilty as the difference of (109) and $q \times(110)$.

$$
\begin{gathered}
\sum_{n=0}^{\infty} \frac{\left(q^{3} ; q^{6}\right)_{n} q^{n^{2}}}{\left(q^{4} ; q^{4}\right)_{n}\left(q ; q^{2}\right)_{n}^{2}} \\
=\prod_{n=1}^{\infty} \frac{\left(1+q^{12 n-2}\right)\left(1+q^{12 n-10}\right)\left(1-q^{12 n}\right)\left(1-q^{24 n-8}\right)\left(1-q^{24 n-16}\right)\left(1+q^{2 n-1}\right)}{1-q^{2 n}}
\end{gathered}
$$

Identity A.110.

$$
\sum_{n=0}^{\infty} \frac{\left(q^{3} ; q^{6}\right)_{n}\left(-q ; q^{2}\right)_{n+1} q^{n(n+2)}}{\left(q^{2} ; q^{2}\right)_{2 n+1}\left(q ; q^{2}\right)_{n}}=\prod_{n=1}^{\infty} \frac{\left(1-q^{12 n}\right)\left(1+q^{2 n-1}\right)}{1-q^{2 n}}
$$

\section{Identity A.111.}

$$
1+\sum_{n=1}^{\infty} \frac{\left(q^{6} ; q^{6}\right)_{n-1}\left(-q ; q^{2}\right)_{n} q^{n(n+2)}}{\left(q^{2} ; q^{2}\right)_{2 n}\left(q^{2} ; q^{2}\right)_{n-1}}=\frac{\left(q^{15}, q^{21}, q^{36} ; q^{36}\right)_{\infty}-q\left(q^{9}, q^{27}, q^{36} ; q^{36}\right)_{\infty}}{\left(q^{4} ; q^{4}\right)_{\infty}\left(q ; q^{2}\right)_{\infty}}
$$

Identity A.112. Note: This identity is (115) $+q^{3} \times(116)$.

$$
\sum_{n=0}^{\infty} \frac{\left(q^{6} ; q^{6}\right)_{n}\left(-q ; q^{2}\right)_{n+2} q^{n(n+2)}}{\left(q^{2} ; q^{2}\right)_{2 n+2}\left(q^{2} ; q^{2}\right)_{n}}=\frac{\left(q^{9}, q^{27}, q^{36} ; q^{36}\right)_{\infty}+q^{3}\left(q^{9}, q^{3}, q^{33} ; q^{36}\right)_{\infty}}{\left(q^{4} ; q^{4}\right)_{\infty}\left(q ; q^{2}\right)_{\infty}}
$$

Identity A.113. Note: This identity is (115) $-q^{3} \times(116)$.

$$
1+\sum_{n=1}^{\infty} \frac{\left(q^{6} ; q^{6}\right)_{n-1}\left(-q ; q^{2}\right)_{n} q^{n(n+2)}}{\left(q^{2} ; q^{2}\right)_{2 n-1}\left(q^{2} ; q^{2}\right)_{n-1}}=\frac{\left(q^{15}, q^{21}, q^{36} ; q^{36}\right)_{\infty}-q^{3}\left(q^{3}, q^{33}, q^{36} ; q^{36}\right)_{\infty}}{\left(q^{4} ; q^{4}\right)_{\infty}\left(q ; q^{2}\right)_{\infty}}
$$

Identity A.114.

$$
1+\sum_{n=1}^{\infty} \frac{\left(q^{6} ; q^{6}\right)_{n-1}\left(-q ; q^{2}\right)_{n} q^{n^{2}}}{\left(q^{2} ; q^{2}\right)_{2 n-1}\left(q^{2} ; q^{2}\right)_{n}}=\prod_{n=1}^{\infty}\left[\left(1-q^{36 n-15}\right)\left(1-q^{36 n-21}\right)\left(1-q^{36 n}\right)\right] \frac{1+q^{2 n-1}}{1-q^{2 n}}
$$

Identity A.115 (Bailey-Dyson). Bailey [21, p. 435, (C3)].

$$
\sum_{n=0}^{\infty} \frac{\left(q^{6} ; q^{6}\right)_{n}\left(-q ; q^{2}\right)_{n+1} q^{n(n+2)}}{\left(q^{2} ; q^{2}\right)_{2 n+2}\left(q^{2} ; q^{2}\right)_{n}}=\prod_{n=1}^{\infty}\left[\left(1-q^{36 n-9}\right)\left(1-q^{36 n-27}\right)\left(1-q^{36 n}\right)\right] \frac{1+q^{2 n-1}}{1-q^{2 n}}
$$


Identity A.116 (Bailey-Dyson). Bailey [21, p. 435, (C1)].

$$
\sum_{n=0}^{\infty} \frac{\left(q^{6} ; q^{6}\right)_{n}\left(-q ; q^{2}\right)_{n+1} q^{n(n+4)}}{\left(q^{2} ; q^{2}\right)_{2 n+2}\left(q^{2} ; q^{2}\right)_{n}}=\prod_{n=1}^{\infty}\left[\left(1-q^{36 n-3}\right)\left(1-q^{36 n-33}\right)\left(1-q^{36 n}\right)\right] \frac{1+q^{2 n-1}}{1-q^{2 n}}
$$

\section{Identity A.117.}

$$
\begin{gathered}
\sum_{n=0}^{\infty} \frac{\left(-q ; q^{2}\right)_{n} q^{n^{2}}}{\left(q^{2} ; q^{2}\right)_{2 n}} \\
=\prod_{n=1}^{\infty}\left[\left(1-q^{14 n-3}\right)\left(1-q^{14 n-11}\right)\left(1-q^{14 n}\right)\left(1-q^{28 n-8}\right)\left(1-q^{28 n-20}\right)\right] \frac{1+q^{2 n-1}}{1-q^{2 n}}
\end{gathered}
$$

\section{Identity A.118.}

$$
\begin{gathered}
\sum_{n=0}^{\infty} \frac{\left(-q ; q^{2}\right)_{n} q^{n(n+2)}}{\left(q^{2} ; q^{2}\right)_{2 n}} \\
=\prod_{n=1}^{\infty}\left[\left(1-q^{14 n-1}\right)\left(1-q^{14 n-13}\right)\left(1-q^{14 n}\right)\left(1-q^{28 n-12}\right)\left(1-q^{28 n-16}\right)\right] \frac{1+q^{2 n-1}}{1-q^{2 n}}
\end{gathered}
$$

Identity A.119.

$$
\begin{gathered}
\sum_{n=0}^{\infty} \frac{\left(-q ; q^{2}\right)_{n+1} q^{n(n+2)}}{\left(q^{2} ; q^{2}\right)_{2 n+1}} \\
=\prod_{n=1}^{\infty}\left[\left(1-q^{14 n-5}\right)\left(1-q^{14 n-9}\right)\left(1-q^{14 n}\right)\left(1-q^{28 n-4}\right)\left(1-q^{28 n-24}\right)\right] \frac{1+q^{2 n-1}}{1-q^{2 n}}
\end{gathered}
$$

\section{Identity A.120.}

$$
1+\sum_{n=1}^{\infty} \frac{\left(-q^{2} ; q^{2}\right)_{n-1} q^{n(n+1)}}{(q ; q)_{2 n}}=\frac{\left(-q^{22},-q^{26}, q^{48} ; q^{48}\right)_{\infty}-q\left(-q^{14},-q^{34}, q^{48} ; q^{48}\right)_{\infty}}{(q ; q)_{\infty}}
$$

Identity A.121.

$$
\begin{gathered}
1+\sum_{n=1}^{\infty} \frac{\left(-q^{2} ; q^{2}\right)_{n-1} q^{n^{2}}}{(q ; q)_{2 n}} \\
=\prod_{n=1}^{\infty} \frac{\left(1-q^{16 n-2}\right)\left(1-q^{16 n-14}\right)\left(1-q^{16 n}\right)\left(1-q^{32 n-12}\right)\left(1-q^{32 n-20}\right)}{1-q^{n}}
\end{gathered}
$$

Identity A.122.

$$
\sum_{n=0}^{\infty} \frac{\left(-q^{2} ; q^{2}\right)_{n} q^{n(n+3)}}{(q ; q)_{2 n+2}}=\frac{\left(-q^{10},-q^{26}, q^{38} ; q^{48}\right)_{\infty}-q^{3}\left(-q^{2},-q^{46}, q^{48} ; q^{48}\right)_{\infty}}{(q ; q)_{\infty}}
$$




\section{Identity A.123.}

$$
\begin{gathered}
\sum_{n=0}^{\infty} \frac{\left(-q^{2} ; q^{2}\right)_{n} q^{n(n+2)}}{(q ; q)_{2 n+2}} \\
=\prod_{n=1}^{\infty} \frac{\left(1-q^{16 n-6}\right)\left(1-q^{16 n-10}\right)\left(1-q^{16 n}\right)\left(1-q^{32 n-4}\right)\left(1-q^{32 n-28}\right)}{1-q^{n}}
\end{gathered}
$$

Identity A.124.

$$
\begin{gathered}
\sum_{n=0}^{\infty} \frac{\left(q^{3} ; q^{6}\right)_{n} q^{2 n(n+1)}}{\left(q^{2} ; q^{2}\right)_{2 n+1}\left(q ; q^{2}\right)_{n}} \\
=\prod_{n=1}^{\infty} \frac{\left(1+q^{18 n-5}\right)\left(1+q^{18 n-13}\right)\left(1-q^{18 n}\right)\left(1-q^{36 n-8}\right)\left(1-q^{36 n-28}\right)}{1-q^{2 n}}
\end{gathered}
$$

Identity A.125.

$$
\begin{gathered}
\sum_{n=0}^{\infty} \frac{\left(q^{3} ; q^{6}\right)_{n} q^{2 n(n+2)}}{\left(q^{2} ; q^{2}\right)_{2 n+1}\left(q ; q^{2}\right)_{n}} \\
=\prod_{n=1}^{\infty} \frac{\left(1+q^{18 n-7}\right)\left(1+q^{18 n-11}\right)\left(1-q^{18 n}\right)\left(1-q^{36 n-4}\right)\left(1-q^{36 n-32}\right)}{1-q^{2 n}}
\end{gathered}
$$

Identity A.126. Note: This identity is equivalent to $(71)+q \times(68)-q \times(128)$.

$$
1+\sum_{n=1}^{\infty} \frac{\left(-q^{4} ; q^{4}\right)_{n-1}\left(-q ; q^{2}\right)_{n} q^{n^{2}}}{\left(q^{2} ; q^{2}\right)_{2 n}}=\frac{\left(-q^{28},-q^{36}, q^{64} ; q^{64}\right)_{\infty}-q^{3}\left(-q^{12},-q^{52}, q^{64} ; q^{64}\right)_{\infty}}{\left(q ; q^{2}\right)_{\infty}\left(q^{4} ; q^{4}\right)_{\infty}}
$$

Identity A.127. Note: This identity is equivalent to $(71)-q \times(128)$.

$$
1+\sum_{n=1}^{\infty} \frac{\left(-q^{4} ; q^{4}\right)_{n-1}\left(-q ; q^{2}\right)_{n} q^{n(n+2)}}{\left(q^{2} ; q^{2}\right)_{2 n}}=\frac{\left(-q^{28},-q^{36}, q^{64} ; q^{64}\right)_{\infty}-q\left(-q^{20},-q^{44}, q^{64} ; q^{64}\right)_{\infty}}{\left(q ; q^{2}\right)_{\infty}\left(q^{4} ; q^{4}\right)_{\infty}}
$$

\section{Identity A.128.}

$$
\sum_{n=0}^{\infty} \frac{\left(-q^{4} ; q^{4}\right)_{n}\left(-q ; q^{2}\right)_{n+1} q^{n(n+2)}}{\left(q^{2} ; q^{2}\right)_{2 n+2}}=\frac{\left(-q^{20},-q^{44}, q^{64} ; q^{64}\right)_{\infty}-q^{5}\left(-q^{4},-q^{60}, q^{64} ; q^{64}\right)_{\infty}}{\left(q ; q^{2}\right)_{\infty}\left(q^{4} ; q^{4}\right)_{\infty}}
$$

Identity A.129. Note: This identity is equivalent to $q^{-2} \times((128)-(68))$.

$$
\sum_{n=0}^{\infty} \frac{\left(-q^{4} ; q^{4}\right)_{n}\left(-q ; q^{2}\right)_{n+1} q^{n(n+4)}}{\left(q^{2} ; q^{2}\right)_{2 n+2}}=\frac{\left(-q^{12},-q^{52}, q^{64} ; q^{64}\right)_{\infty}-q^{3}\left(-q^{4},-q^{60}, q^{64} ; q^{64}\right)_{\infty}}{\left(q ; q^{2}\right)_{\infty}\left(q^{4} ; q^{4}\right)_{\infty}}
$$


Identity A.130. Note: This identity is $(72-a)-q \times(70)$.

$$
\sum_{n=0}^{\infty} \frac{\left(-q^{2} ; q^{4}\right)_{n}\left(-q ; q^{2}\right)_{n+1} q^{n^{2}}}{\left(q^{2} ; q^{2}\right)_{2 n+1}}=\frac{\left(q^{8}, q^{8}, q^{16} ; q^{16}\right)_{\infty}-q\left(q^{4}, q^{12}, q^{16} ; q^{16}\right)_{\infty}}{\left(q ; q^{2}\right)_{\infty}\left(q^{4} ; q^{4}\right)_{\infty}}
$$

\section{Acknowledgements}

First and foremost, I thank my thesis advisor George E. Andrews . I am also grateful to Alexander Berkovich, Paul Eakin, Peter Paule, Avinash Sathaye, and Doron Zeilberger for their support and encouragement of this project.

\section{References}

[1] H. L. Alder. Partition identites: From Euler to the present. Amer. Math. Monthly, 76(7):733-746, August-September 1969.

[2] American Mathematical Society. 1998 steele prizes. Notices Amer. Math. Soc., 45(504):504-508, March 1998.

[3] G. E. Andrews. A polynomial identity which implies the Rogers-Ramanujan identities. Scripta Mathematica, 28:297-305, 1970.

[4] G. E. Andrews. Number Theory. W. B. Saunders, Philadelphia, 1971. Reissued by Dover, 1994.

[5] G. E. Andrews. On Rogers-Ramanujan type identities related to the modulus 11. Proc. London Math. Soc., 30:330-346, 1975.

[6] G. E. Andrews. The Theory of Partitions, volume 2 of Encyclopedia of Mathematics and Its Applications. Addison-Wesley, Reading, MA, 1976.

[7] G. E. Andrews. The hard-hexagon model and Rogers-Ramanujan type identities. Proc. Nat. Acad. Sci. USA, 78(9):5290-5292, September 1981.

[8] G. E. Andrews. Multiple series Rogers-Ramanujan type identities. Pacific J. Math., 114(2):267-283, 1984.

[9] G. E. Andrews. Combinatorics and Ramanujan's "lost" notebook. In Sureys in Combinatorics, volume 103 of London Mathematical Society Lecture Note Series, pages 1-23. London Mathematical Society, 1985.

[10] G. E. Andrews. q-Series: Their Development and Application in Analysis, Number Theory, Combinatorics, Physics, and Computer Algebra, volume 66 of Regional Conference Series in Mathematics. American Mathematical Society, Providence, RI, 1986. 
[11] G. E. Andrews. Rogers-Ramanujan identities for two-color partitions. Indian J. Math., 29(2):117-125, 1987. Ramanujan Centenary Volume.

[12] G. E. Andrews. Euler's "Exemplum memorabile inductionis fallacis" and $q$-trinomial coefficients. J. Amer. Math. Soc., 3(3):653-669, 1990.

[13] G. E. Andrews. q-trinomial coefficients and Rogers-Ramanujan type identities. In Bruce Berndt et al., editor, Analytic Number Theory, pages 1-11, Boston, 1990. Birkhauser.

[14] G. E. Andrews, R. Askey, and R. Roy. Special Functions, volume 71 of Encyclopedia of Mathematics and Its Applications. Cambridge University Press, 1999.

[15] G. E. Andrews and R. J. Baxter. Lattice gas generalization of the hard hexagon model III: q-trinomial coefficients. J. Statist. Phys., 47(3/4):297-330, 1987.

[16] G. E. Andrews and R. J. Baxter. Scratchpad explorations for elliptic theta functions. In David V. Chudnovsky and Richard D. Jenks, editors, Computers in Mathematics, volume 125 of Lecture Notes in Pure and Applied Mathematics, pages 17-33, 270 Madison Avenue, New York, NY 10016, 1990. Marcel Dekker, Inc.

[17] G. E. Andrews, R. J. Baxter, D. M Bressoud, W. H. Burge, P. J. Forrester, and G. Viennot. Partitions with prescribed hook differences. Europ. J. Combinatorics, 8:341-350, 1984.

[18] G. E. Andrews, R. J. Baxter, and P. J. Forrester. Eight vertex SOS model and generalized Rogers-Ramanujan type identities. J. Statist. Phys., 35:193-266, 1984.

[19] G. E. Andrews and A. Berkovich. A trinomial analogue of Bailey's lemma and $N=2$ superconformal invariance. Comm. Math. Phys., 192:245-260, 1998.

[20] G. E. Andrews and J. P. O. Santos. Rogers-Ramanujan type identities for partitions with attached odd parts. Ramanujan J., (1):91-99, January 1997.

[21] W. N. Bailey. Some identities in combinatory analysis. Proc. London Math. Soc., 49:421-435, 1947.

[22] W. N. Bailey. Identities of the Rogers-Ramanujan type. Proc. London Math. Soc., 50:1-10, 1949.

[23] W. N. Bailey. On the simplification of some identities of the Rogers-Ramanujan type. Proc. London Math. Soc., 1:217-221, 1951.

[24] R. J. Baxter. Hard hexagons: exact solution. J. Phys. A, 13:L61-L70, 1980.

[25] R. J. Baxter. Rogers-Ramanujan identities in the hard hexagon model. J. Statist. Phys., 26:427-452, 1981. 
[26] R. J. Baxter. Exactly Solved Models in Statistical Mechanics. Academic Press, London and New York, 1982.

[27] A. Berkovich and B. M. McCoy. Continued fractions and fermionic representations for characters of $M\left(p, p^{\prime}\right)$ minimal models. Lett. Math. Phys., 37:49-66, 1996.

[28] A. Berkovich and B. M. McCoy. Generalizations of the Andrews-Bressoud identities for the $N=1$ superconformal model $S M(2,4 \nu)$. Math. Comput. Modelling, 26(810):37-49, 1997.

[29] A. Berkovich and B. M. McCoy. Rogers-Ramanujan identities: A century of progress from mathematics to physics. Doc. Math., Extra volume ICM III:163-172, 1998.

[30] A. Berkovich, B. M. McCoy, and W. P. Orrick. Polynomial identities, indices, and duality for the $N=1$ superconformal model $S M(2,4 \nu)$. J. Statist. Phys., 83:795-837, 1996.

[31] A. Berkovich, B. M. McCoy, and A. Schilling. Rogers-Schur-Ramanujan type identities for the $M\left(p, p^{\prime}\right)$ minimal models of conformal field theory. Comm. Math. Phys., 191:211-223, 1998.

[32] D. M. Bressoud. Some identities for terminating q-series. Math. Proc. Cambridge Philos. Soc., 89:211-223, 1981.

[33] A.-L. Cauchy. Mémoire sur les fonctions dont plusierus valeurs... C. R. Acad. Sci. Paris, 17:523, 1843.

[34] E. Data, J. Jimbo, A. Kuniba, T. Miwa, and M. Okado. Exactly solvable SOS models: local height probabilities and theta function identities. Nuclear Phys. B, 290:231-273, 1987.

[35] L. Euler. Introduction to analysis of the infinite, volume 1. Springer-Verlag, 1988. Translation of Introductio in analysin infinitorum, 1748.

[36] P. J. Forrester and R. J. Baxter. Further exact solutions of the eight vertex SOS model and generalized Rogers-Ramanujan type identities. J. of Statist. Phys., 38:435-472, 1985.

[37] G. Gasper and M. Rahman. Basic hypergeometric series, volume 35 of Encyclopedia of Mathematics and its Applications. Cambridge University Press, New York, 1990.

[38] K. F. Gauss. Summatio serierum quarumdam singularium, volume 1. Göttingen, 1808-1811.

[39] K. F. Gauss. Zur Theorie der Neuen Transscendenten II: Zur Theorie der transsendenten Functionen Gehörig, volume 3 of Werke. Königlichen Gesellschaft der Wissenschaften, Göttingen, 1876. 
[40] H. Göllnitz. Einfache partitionen. Diplomarbeit W. S., Göttingen, 1960.

[41] B. Gordon. A combinatorial generalization of the Rogers-Ramanujan identities. Amer. J. Math., 83:393-399, 1961.

[42] B. Gordon. Some continued fractions of the Rogers-Ramanujan type. Duke J. Math., 31:741-748, 1965.

[43] G. H. Hardy. Ramanujan: Twelve lectures suggested by his life and work. Chelsea, third edition, 1978. Originally published Cambridge: Cambridge University Press, 1940. Reprinted by the American Mathematical Society, 1999 with corrections and comments.

[44] H. E. Heine. Untersuchugen über die reihe... Journal Reine Angew. Math., 34:285$328,1847$.

[45] M. E. H. Ismail. A simple proof of Ramanujan's ${ }_{1} \psi_{1}$ sum. Proc. Amer. Math. Soc., 63:185-186, 1977.

[46] F. H. Jackson. Examples of a generalization of Euler's transformation for power series. Messenger of Mathematics, 57:169-187, 1928.

[47] T. H. Koornwinder. On Zeilberger's algorithm and its q-analog. J. Comput. Appl. Math., 48:91-111, 1993.

[48] V. A. Lebesgue. Sommation de quelques séries. Journal Math. Pures Appl., 5:42-71, 1840.

[49] J. Lepowski and R. L. Wilson. A Lie theoretic interpretation and proof of the RogersRamanujan identities. Adv. Math., 45:199-290, 1982.

[50] P. A. MacMahon. Combinatory Analysis, volume 2. Cambridge University Press, London, 1918.

[51] P. Paule. Zwei neue Transformationen als elementare Anwendungen der qVandermonde Formel. PhD thesis, University of Vienna, 1982.

[52] P. Paule. On identities of the Rogers-Ramanujan type. J. Math. Anal. Appl., 107(1):255-284, April 1985.

[53] P. Paule. Short and easy computer proofs of the Rogers-Ramanujan identities and of identities of similar type. Electron. J. Combin., 1, 1994. \# R10.

[54] P. Paule and A. Riese. A Mathematica q-analogue of Zeilberger's algorithm based on an algebraically motivated approach to $q$-hypergeometric telescoping. In Special Functions, q-series and Related Topics, volume 14 of Fields Institute Communications, pages 179-210, Providence, RI, 1997. American Mathematical Society. 
[55] M. Petkovšek, H. S. Wilf, and D. Zeilberger. $A=B$. A. K. Peters, Wellesley, MA, 1996.

[56] A. Riese. q-MultiSum - a package for proving $q$-hypergeometric multiple summation identities. J. Symbolic Comput., 35:349-376, 2003.

[57] L. J. Rogers. Second memoir on the expansion of certain infinite products. Proc. London Math. Soc., 25:318-343, 1894.

[58] L. J. Rogers. On two theorems of combinatory analysis and some allied identities. Proc. London Math. Soc., 16:315-336, 1917.

[59] H. A. Rothe. Systematisches Lehrbuch der Arithmetic. Leipzig, 1811.

[60] J. P. O. Santos. Computer Algebra and Identities of the Rogers-Ramanujan Type. PhD thesis, The Pennsylvania State University, 1991.

[61] J. P. O Santos. On the combinatorics of polynomial generalizations of RogersRamanujan type identities. Discrete Math., 254:497-511, 2002.

[62] J. P. O. Santos and A. V. Sills. q-pell sequences and two identities of V. A. Lebesgue. Discrete Math., 257:125-142, 2002.

[63] A. Schilling and S. O. Warnaar. Supernomial coefficients, polynomial identities, and $q$-series. Ramanujan J., 2:459-494, 1998.

[64] I. Schur. Ein Beitrag zur additeven Zahlentheorie und zur Theorie der Kettenbrüche. Sitzungsberichte der Berliner Akademie, pages 302-321, 1917.

[65] I. Schur. Zur additiven zahlentheorie. Sitzungsberichte der Berliner Akademie, pages 488-495, 1926.

[66] A. Selberg. Über einige arithmetische Identitäten. Avrandlinger Norske Akad., 8, 1936.

[67] A. V. Sills. RRtools-a Maple package for aiding the discovery and proof of finite Rogers-Ramanujan type identities. J. Symbolic Comput., to appear.

[68] L. J. Slater. Further identities of the Rogers-Ramanujan type. Proc. London Math. Soc., 54:147-167, 1952.

[69] L. J. Slater. Generalized Hypergeometric Functions. Cambridge University Press, 1966.

[70] S. O. Warnaar. The generalized Borwein conjecture II: refined $q$-trinomial coefficients. Preprint.

[71] S. O. Warnaar. q-trinomial identities. J. Math. Phys., 40(4):2514-2530, May 1999. 
[72] S. O. Warnaar. Refined $q$-trinomial coefficients and character identities. J. Stat. Phys., 102, 2001.

[73] G. N. Watson. Theorems stated by Ramanujan VII: Theorems on continued fractions. J. London Math. Soc., 4:39-48, 1929.

[74] K. Weierstraß. Mathematische Werke von Karl Weierstraß, volume II. Mayer \& Müller, Berlin, 1895.

[75] E. T. Whittaker and G. N. Watson. A Course of Modern Analysis. Cambridge University Press, London and New York, fourth edition, 1958.

[76] H. S. Wilf and D. Zeilberger. Rational functions certify combinatorial identities. $J$. Amer. Math. Soc., 3(1):147-158, 1990.

[77] H. S. Wilf and D. Zeilberger. Towards computerized proofs of identities. Bull. Amer. Math. Soc, 23(1):77-83, 1990.

[78] H. S. Wilf and D. Zeilberger. Rational function certification of hypergeometric multiintegral/sum/"q"identities. Invent. Math., 108:575-633, 1992.

[79] D. Zeilberger. A fast algorithm for proving termininating hypergeometric identities. Discrete Math., 80:207-211, 1990.

[80] D. Zeilberger. The method of creative telescoping. J. Symbolic Comput., 11:195-204, 1991. 


\section{Index}

Abel's lemma, 12

Alder, H. L., 14

Andrews, George E., 2, 7, 8, 10, 11, 14, $21,25,26,31-33,35,43,46,47$, $56,57,74,98,99,103,114$

Bailey's mod 9 identities, 103

finite, 33-34

Bailey, W. N., 12, 14, 100, 103, 107-109, 112

Baxter, Rodney, 2, 8, 10, 13, 14, 21

Baxter, Rodney M., 2

Berkovich, Alexander, 2, 4, 7, 14, 21-26, $30-32,39,114$

binomial theorem, 5

bosonic representation, 13

Bressoud, David M., 3, 93, 94

Cauchy, A.-L., 5

creative symmetrization, 61-63

creative telescoping, 59

duality, reciprocal, 74-92, 94

Dyson, Freeman, 107-109, 112

Eakin, Paul, 114

Ekhad, Shalosh, B., 59

Euler's pentagonal number theorem, see Pentagonal Number Theorem

Euler, L., 16, 22, 94, 98

Fasenmyer, Sister Mary Celine, 58

fermionic representation, 13

Forrester, P. J., 2, 14

Gauss, K. F., 5, 99

Gaussian polynomial, 4-5, 18

Gordon, Basil, 14, 102

Göllnitz, H., 14, 102

Göllnitz-Gordon identities, 102 finite, 31-32

Hardy, G. H., 14
Heine's transformation, 12, 28

Heine, E., 5, 12, 28

Jackson, F. H., 14, 99, 100, 103

Jacobi's triple product identity, see triple product identity

Jacobi, K., 11

Koornwinder, T., 58

Lebesgue, V. A., 99

Lepowski, J., 13

MacMahon, P. A., 13, 14, 25, 26

McCoy, Barry M., 2, 4, 14, 21-26, 30-32, 39

operator annihilating, 64 operator algebra, 63

Orrick, William P., 4, 21, 22, 24, 25

partition (of an integer), 13

Paule, Peter, 14, 59-62, 114

Pentagonal Number Theorem, 98

finite, 22,94

Petkovšek, Marko, 58

q-binomial coëfficient, see Gaussian polynomial

q-binomial theorem, 5

q-difference equation, 93

q-difference equations, 14-17

q-factorial, 4

q-trinomial coëfficients, 6-10

asymptotics, 10

definitions, 7

recurrences, 7

qMultiSum (Mathematica package), 59, 66

qZeil (Mathematica package), 59, 60

Ramanujan, S., 13 
recurrence proof, 69-73

Riese, Axel, 59, 60, 66, 94

Rogers, L. J., 13, 14, 100, 102, 103, 105, 107-109

Rogers-Ramanujan identities, 13-14, 92, 100

Bressoud finitization, 93

MacMahon-Schur finitization, 25, 26, 76

Rogers-Selberg identities, 102

finite, 30-31

Rothe, H. A., 6

RRtools (Maple package), 14, 16

Santos, J. P. O., 2, 22, 24, 26-47, 50-54, $56,57,73$

Sathaye, Avinash, 114

Schilling, Anne, 2, 22

Schur, I., 14, 25, 26

Selberg, A., 102

Sills, Andrew V., 14, 16, 73

Slater's list of Rogers-Ramanujan type identities, 14, 98-114

polynomial generalizations of, 21-55

Slater, L. J., 14

Slater, Lucy J., 2, 3, 11, 14, 16, 20-55, 98-114

Steele Prize, 58

theta functions, 11

trinomial coëfficeint q-analog of, see q-trinomial coëfficients

trinomial coëfficient ordinary, 6

triple product identity, 10

Warnaar, S. Ole, 2, 3, 7, 14, 94

Watson, G. N., 12

Weierstraß, K., 12

Wilf, Herbert S., 3, 58, 59

Wilson, Robert L., 13

WZ method, 58-69

Zeilberger's algorithm, 59

Zeilberger, Doron, 3, 58-60, 114 